\title{
Measurement of Neutron and Muon Fluxes 100 m Underground with the SciBath Detector
}

\author{
Lance M. Garrison
}

Submitted to the faculty of the Graduate School

in partial fulfillment of the requirements

for the degree

Doctor of Philosophy

in the Department of Physics,

Indiana University

April 2014 
Accepted by the Graduate Faculty, Indiana University, in partial fulfillment of the requirements of the degree of Doctor of Philosophy.

Doctoral Committee
Rex Tayloe(Chair)

W. Michael Snow

Michael S. Berger

February 6, 2014 
Copyright (c) 2014

Lance Garrison 


\section{Acknowledgements}

I would first like to thank the members of my committee for their time, support, and evaluation of my work. In particular to my adviser, Rex Tayloe, thank you for your guidance and expertise. You assisted me through the vast majority of my graduate career at IU, helped shape this thesis and analysis, and taught me what it is to be a physicist. It has been a privilege to work with you over these years.

I would like to thank Indiana University and the Physics Department and Center for the Exploration of Energy and Matter in particular for making my graduate career possible. To the secretaries and staff, thank you for supporting the work of all graduate students with kindness and patience. I also thank the National Science Foundation for funding this research and the Fermi National Accelerator Laboratory for offering its facilities and expertise.

To those who have contributed to the SciBath detector, I thank you for making this detector and research possible. An incomplete list of those contributors includes Rex Tayloe, Hans-Otto Meyer, Robert Cooper, R. Tyler Thornton, Ethan Steele, Lori Rebenitsch, Melanie Novak, Tyler Mikev, and Robin Macheel. To Robert, thank you for your hard work, enthusiasm, and willingness to offer your time and expertise. Tyler, I thank you for your hard work and for bringing much-needed conversation to our office.

The Foundations in Science and Mathematics summer program has been an important part of my graduate career, and I would like to thank those that made the program possible. In particular, Jake Bennett, Nick Timme, and Jason Fry, thank you for taking the initiative to found the program and for including me from the start. Many others, both graduate student instructors and IU faculty and staff, have assisted with the program, but I would like to thank Adam Maltese, Rick Van Kooten, and Adam Szczepaniak for serving as advisors or principal investigators. This program has been a great success through all of our effort 
and I expect it will continue to serve IU and the community for many years.

To my mother, thank you for believing in me, encouraging me to follow my dreams, and providing me with a foundation from which I could climb to the highest level of education. I would not be where I am today without your support. To my fiancé Amanda, thank you for your patience as you delayed your career and remained at my side through this program. Additionally, thank you for supporting our home and personal responsibilities when my workload peaked. Finally, I would like to thank the Bartlett family, especially Karen, for supporting Amanda and me and for acting as my second family. 


\section{Lance M. Garrison}

Measurement of Neutron and Muon Fluxes100 m Underground with the SciBath Detector

The SciBath detector is an 80 liter liquid scintillator detector read out by a three dimensional grid of 768 wavelength-shifting fibers. Initially conceived as a fine-grained charged particle detector for neutrino studies that could image charged particle tracks in all directions, it is also sensitive to fast neutrons (15-200 MeV). In fall of 2011 the apparatus performed a three month run to measure cosmic-induced muons and neutrons 100 meters underground in the FNAL MINOS near-detector area. Data from this run has been analyzed and resulted in measurements of the cosmic muon flux as $0.80 \pm 0.04 \mathrm{~m}^{-2} \mathrm{~s}^{-1}$, the cosmogenic fast neutron flux as $(1.5 \pm 1.4) \times 10^{-2} \mathrm{~m}^{-2} \mathrm{~s}^{-1}$, and the neutron production rate from muon spallation in liquid scintillator as $(3.8 \pm 3.2) \times 10^{-4} \mathrm{n} / \mu\left(\mathrm{g} / \mathrm{cm}^{2}\right)^{-1}$. Additionally, the cosmic muon angular distribution and neutron energy distribution have been measured. These results can be extrapolated to future measurements of fast-neutron backgrounds at other underground facilities. This thesis presents a summary of the physics relevant to underground muons and fast neutrons, the SciBath detector, the analysis methodology that was used for the results presented, and comparisons between our results and those of other experiments and simulations.

Rex Tayloe(Chair)

W. Michael Snow

Michael S. Berger

Sima Setayeshgar 


\section{Contents}

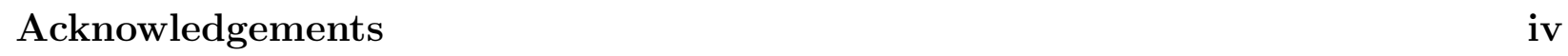

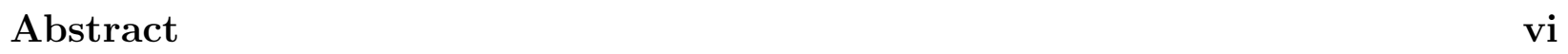

$\begin{array}{lll}1 & \text { Introduction } & 1\end{array}$

1.1 Structure of this thesis . . . . . . . . . . . . . . . . . . . . . . 3

2 Muons and Neutrons Underground 4

2.1 Muons underground . . . . . . . . . . . . . . . . . . . . . . . . . . . . . . . . . .

2.2 Muons in SciBath . . . . . . . . . . . . . . . . . . . . . . . . . 6

2.2 .1 Minimum ionizing muons . . . . . . . . . . . . . . . . . . . . . 6

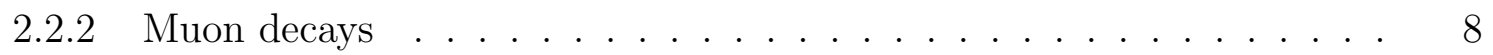

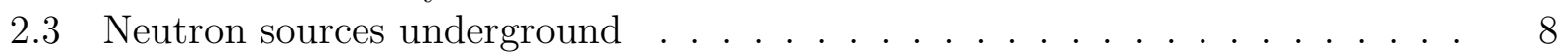

2.3 .1 Hadronic component of cosmic rays . . . . . . . . . . . . . . . . . . . 9

2.3 .2 Radioactivity in the earth . . . . . . . . . . . . . . . . . . . . 10

2.3 .3 Neutron production through muon spallation . . . . . . . . . . . . . . 10

2.3 .4 Muon capture . . . . . . . . . . . . . . . . . . . . . . 12

2.4 Neutron interactions in SciBath . . . . . . . . . . . . . . . . . . . . . 14

2.4 .1 Elastic scattering . . . . . . . . . . . . . . . . . . . . . . . . . . . . . . . . . . . . . . .

2.4 .2 Radiative capture . . . . . . . . . . . . . . . . . . . . 16

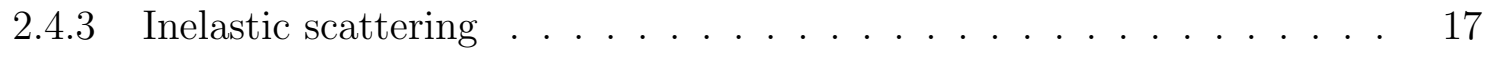

2.4 .4 A typical fast neutron in SciBath . . . . . . . . . . . . . . . . . . 17

2.5 Photons in SciBath . . . . . . . . . . . . . . . . . . . . . . . . . . . . 19

$3 \quad$ Previous Measurements and Simulations $\quad 22$

3.1 Muon flux and angular distribution . . . . . . . . . . . . . . . . . . . . 22

3.2 Neutron production rate through muon spallation . . . . . . . . . . . . . . 25

3.3 Neutron flux and energy distribution . . . . . . . . . . . . . . . . . . 27

$\begin{array}{lll}4 \text { The SciBath Detector } & 30\end{array}$

4.1 FINeSSE . . . . . . . . . . . . . . . . . . . . . . . . . . 30

4.2 Overview and principle of operation . . . . . . . . . . . . . . . . . . . . . . . . . . . . . . . . 31

4.3 Scintillator . . . . . . . . . . . . . . . . . . . . 33

4.3 .1 Notes on mixing . . . . . . . . . . . . . . . . . . . . . . . . . . . . . . . . . . . 35

4.4 Wavelength shifting fibers . . . . . . . . . . . . . . . . 36 
4.5 Optical fibers . . . . . . . . . . . . . . . . . . . . . . . . . . . 37

4.6 Data acquisition system $\ldots \ldots \ldots$. . . . . . . . . . . . . . . . . 37

4.6 .1 Photomultiplier tubes . . . . . . . . . . . . . . . . . . . . . . . . . . . . . . . . . . . . . . .

4.6 .2 Integrated Readout Modules . . . . . . . . . . . . . . . . . . . . . . . 39

4.6 .3 Trigger modes . . . . . . . . . . . . . . . . . . . . . . . . . . . . . . . 42

4.6 .4 Break-in board . . . . . . . . . . . . . . . . . . . . . . . . . 42

4.6 .5 Integrated Readout Module clock synchronization . . . . . . . . . . . 43

4.7 LED calibration system . . . . . . . . . . . . . . . . . . . . . . . . . . 43

4.8 Plumbing $\ldots \ldots \ldots \ldots \ldots$

4.8 .1 Liquid scintillator plumbing . . . . . . . . . . . . . . . . . . . . . . . . . . . . . . . . . . . . 45

4.8 .2 Nitrogen plumbing . . . . . . . . . . . . . . . . . . . . . . . . . . . . . . . . . . . . 48

4.9 Monitoring . . . . . . . . . . . . . . . . . . . . . . 48

5 The NuMI Run $\quad 54$

5.1 The MINOS near detector hall . . . . . . . . . . . . . . . . . . . . . . . . 54

5.2 The NuMI beam . . . . . . . . . . . . . . . . . . . . . . . . . . . 56

5.3 NuMI run summary . . . . . . . . . . . . . . . . . . . . 56

5.3 .1 NuMI beam output summary . . . . . . . . . . . . . . . . . . . . . . . . . . . . 57

5.4 The MI-12 run . . . . . . . . . . . . . . . . . . . . . . . . . . 57

$6 \quad$ Analysis Methodology 59

6.1 Event building . . . . . . . . . . . . . . . . . . . . . . 60

6.1 .1 Waveform fitting . . . . . . . . . . . . . . . . . . . 60

6.1 .2 Photoelectron calibration . . . . . . . . . . . . . . . . . . 61

6.1 .3 Event grouping . . . . . . . . . . . . . . . . . . . . . . . . . . . . . . . . . . . . . 63

6.1 .4 Multiplicity . . . . . . . . . . . . . . . . . . . . . 65

6.2 Timing analysis $\ldots \ldots \ldots$. . . . . . . . . . . . . . . 66

6.2 .1 Cosmic event timing . . . . . . . . . . . . . . . . . . . . . . . . . . . . . . . . 67

6.2 .2 Beam correlated event timing . . . . . . . . . . . . . . . . . . . 67

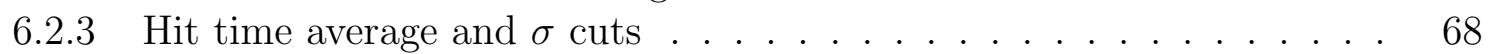

6.3 Energy analysis . . . . . . . . . . . . . . . . . . . . . 68

$6.3 .1 \quad$ Calibration based on minimum ionizing muons . . . . . . . . . . . . . 70

6.3.2 Quenching of proton response . . . . . . . . . . . . . . . . 71

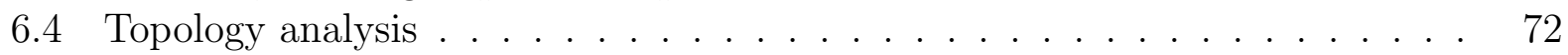

6.4 .1 Required energy response in three dimensions . . . . . . . . . . . . . 72

6.4 .2 Fiducial volume cuts . . . . . . . . . . . . . . . . . . 74

6.4 .3 Eigenvalue analysis for SciBath topology . . . . . . . . . . . . . . . . 74

6.5 Analysis cut summary . . . . . . . . . . . . . . . . . . . . . . . . . . . . . . . . . . . .

6.6 Background subtraction . . . . . . . . . . . . . . . . . . . 78

6.6 .1 Uncorrelated neutron capture candidate rate . . . . . . . . . . . . . . 78

6.6 .2 Incident neutron event counting . . . . . . . . . . . . . . . . . . 79

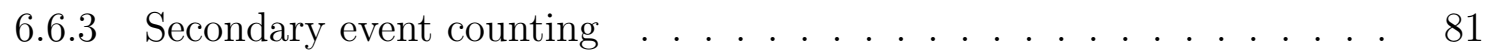

$6.6 .4 \quad$ Extension to energy and time distributions . . . . . . . . . . . . . . . 82

6.7 Monte Carlo simulation . . . . . . . . . . . . . . . . . . . . 83

6.7 .1 Geant4 Monte Carlo simulation . . . . . . . . . . . . . . . . . . . 84 
6.7 .2 MCNP Monte Carlo simulation . . . . . . . . . . . . . . . . . . . . . 86

$6.7 .3 \quad$ Simulation of neutron capture tagging $\ldots \ldots \ldots$. . . . . . . . . 87

$6.7 .4 \quad$ Externally generated neutron detection efficiency . . . . . . . . . . . 87

6.7 .5 Internally generated neutron detection efficiency . . . . . . . . . . . . 91

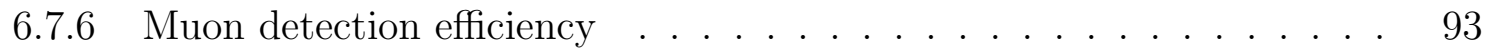

6.7 .7 Muon track length . . . . . . . . . . . . . . . . . . . . . . . . . 94

6.7 .8 Muon average position . . . . . . . . . . . . . . . . . . . . . 95

\begin{tabular}{lll}
\hline & Results & 98
\end{tabular}

7.1 Error estimation $\ldots \ldots \ldots$. . . . . . . . . . . . . . . . . . . . . . . . . . . . . 99

7.1.1 Statistical error . . . . . . . . . . . . . . . . . . . . . . 100

7.1 .2 Systematic error . . . . . . . . . . . . . . . . . 100

7.2 Cosmic muon flux . . . . . . . . . . . . . . . . . . . . . . . . . . . 105

7.3 Cosmic muon angular distribution . . . . . . . . . . . . . . . . . . . . . 106

7.4 Muon-induced neutron spallation in scintillator . . . . . . . . . . . . . . . . 107

7.5 Cosmic neutron flux $\ldots \ldots \ldots \ldots$. . . . . . . . . . . . . . . . . . . . . . . . 114

7.6 Cosmic neutron energy and time distributions . . . . . . . . . . . . . . 117

7.7 neutron / muon ratio . . . . . . . . . . . . . . . . . . . 118

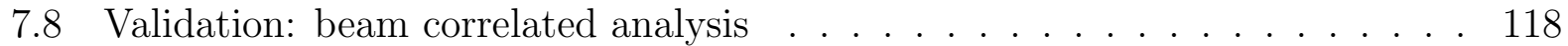

7.9 $\quad$ Implications for EXO . . . . . . . . . . . . . . . . . . . . . . . . . . 123

7.9 .1 Neutrons as a background for EXO . . . . . . . . . . . . . . . . . . 125

7.9 .2 Implications of our measurements . . . . . . . . . . . . . . . . . . 126

8 Conclusion and Outlook $\quad 129$

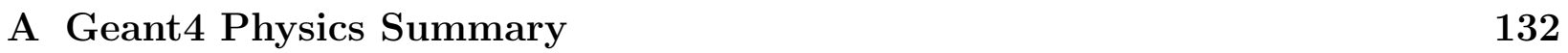

\begin{tabular}{|l|l}
\hline B MCNP Template & 134
\end{tabular}

C Waveform Fitting with the Least Squares Parabola Method 140

List of Symbols . . . . . . . . . . . . . . . . . . . . . . . . . . . . 142

C.1 Background . . . . . . . . . . . . . . . . . . . . . . . . . . 142

C.2 Implementation . . . . . . . . . . . . . . . . . . . . . . . . . . 143

C.3 Results . . . . . . . . . . . . . . . . . . . . . . 145 


\section{List of Tables}

2.1 Parameters for Eqn. $[2.2$ as calculated by Groom et al. [1] and resulting muon ranges in standard rock . . . . . . . . . . . . . . . . . . 6

3.1 World measurements of neutron yield in liquid scintillator. The ${ }^{\dagger}$ indicates measurements that were corrected by Agafonova and Malgin [2].] . . . . . . . 27

3.2 Mean muon energy at WIPP and parameters relevant to its calculation with Eqn,3.7. . . . . . . . . . . . . . . . . . . . . 28

6.1 Event identification cuts summary. Since event timing cuts are only relevant for neutron capture events, they have not been included, see Sec. 6.2.1. . . . . 77

6.2 Summary of the external neutron detection efficiency. The "neutron enter detector" efficiency is specific to the simulation used and is for an assumed SciBath cross section of $\pi(40 \mathrm{~cm})^{2}$, larger than the detector cube. . . . . . . . 89

6.3 Summary of the internal neutron detection efficiency. . . . . . . . . . . . . . 92

7.1 Summary of the errors for all measurements. Those marked with $\mathrm{a}^{\dagger}$ are primarily or entirely statistical; all others are systematic. The indented entries contribute to the preceding "total" error. Total errors were found by addition in quadrature. . . . . . . . . . . . . . . . . . . . . . 99

7.2 Muon flux calculation summary. The error on $A$ is listed as zero because it is included in the error of $\epsilon_{\mu} . \ldots \ldots \ldots \ldots$. . . . . . . . . . . . . . . . . . . . . 106

7.3 Muon-induced neutron production calculation summary. . . . . . . . . . . . 109

7.4 Muon-induced neutron production correction factor, $Q$, calculation summary. 110

7.5 Summary of the detection efficiency for neutrons generated in the detector walls. . . . . . . . . . . . . . . . . . 110

7.6 Mean muon energy at WIPP and NuMI and parameters relevant to their calculation with Eqn $\mid 3.7$. . . . . . . . . . . . . . . . . . . . . . 111

7.7 World measurements of neutron yield in liquid scintillator, including this result. The ${ }^{\dagger}$ indicates measurements that were altered from the original by Ref. [2]. $\ldots \ldots \ldots \ldots \ldots \ldots . \ldots 111$

7.8 Neutron flux calculation summary. The error on $A$ is listed as zero because it

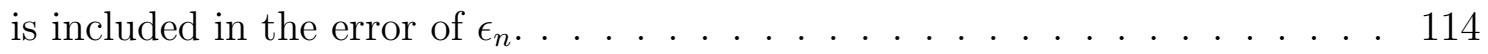

7.9 Neutron flux correction, $N_{n, \mu}^{\prime}$, calculation summary. . . . . . . . . . . . . . . 115 


\section{List of Figures}

2.1 Stopping power $(\langle-d E / d x\rangle)$ for positive muons in copper as a function of kinetic energy, $T$, over 12 orders of magnitude. Solid curves indicate the total stopping power. Data below the break at $T \approx 0.5 \mathrm{MeV}$ are taken from ICRU 49 [3], and data at higher energies are from Ref. [1]. Vertical bands indicate boundaries between different theoretical approximations or dominant physical processes. The short dotted lines labeled " $\mu$ " illustrate the "Barkas effect," the dependence of stopping power on projectile charge at very low energies [4, 5]. "Nuclear losses" indicates non-ionizing nuclear recoil energy losses. Figure from Groom et al. [1].] . . . . . . . . . . . . . . . . . 7

2.2 Total neutron fluxes for different sources as a function of depth in meters water equivalent. At NuMI depth only neutrons from muons contribute to the flux above $16 \mathrm{MeV}$. Figure adapted from the Ph.D. thesis of E.-I. Esch [6].] 9

2.3 Schematic of neutron production through muon spallation inside the detector. SciBath detects the muon followed by a $2.2 \mathrm{MeV}$ photon at a characteristic time near 160 us. . . . . . . . . . . . . . . . . . 11

2.4 The expected total muon-induced neutron yield in liquid scintillator (solid) as a function of depth, and the contribution from muon capture (dotted). Figure adapted from Malgin and Ryazhskaya [7]. . . . . . . . . . . . . . . . . 13

2.5 Cross sections for neutron interactions with hydrogen and carbon. The inelastic portion of the $\mathrm{n}-\mathrm{C}$ total cross section is only noticeable above $10 \mathrm{MeV}$. This data is from ENDF/B-VII.1 tables [8]. . . . . . . . . . . . . . . . . 15

2.6 Schematic of neutron-proton elastic scattering event in SciBath, which is sensitive to the recoiling proton. . . . . . . . . . . . . . . . . . 15

2.7 Neutron capture on a hydrogen nucleus in SciBath. SciBath is sensitive to the characteristic $2.2 \mathrm{MeV}$ photon. . . . . . . . . . . . . . . . . 16

2.8 n-C total, n-C inelastic and n-p total cross sections data. The full lines show the cross sections used in the Monte Carlo program of Del Guerra [9]. Figure by Del Guerra. . . . . . . . . . . . . . . . . . . . . 18

2.9 Cross sections for major neutron on carbon inelastic processes. Figure from Anghinolfi et al. [10]. . . . . . . . . . . . . . . . . 18

2.10 Neutron that scatters off of a hydrogen nucleus, thermalizes, and captures in SciBath. SciBath detects the recoiling proton followed by a $2.2 \mathrm{MeV}$ photon at a characteristic time near $160 \mu \mathrm{s}$. 
2.11 Cross sections for dominant photon interactions in liquid scintillator $\left(\mathrm{CH}_{2}\right)$. Incoherent Compton scattering is the dominant interaction for $2.2 \mathrm{MeV}$ photons from radiative neutron capture on hydrogen. Plot from the NIST XCOM database [1]. . . . . . . . . . . . . . . . . . . . . . . . . . . . . . . . . . . . . 21

3.1 Absolute vertical muon intensity vs. depth measured from the top of the atmosphere. Diagram altered from Dragić et al. [12]. The intensity is scaled by $\mathrm{d}^{3}$ to more clearly show the results. The depth unit hg $/ \mathrm{cm}^{2}$ represents the product of material density and muon path length; and when expressed as $\mathrm{hg} / \mathrm{cm}^{2}$ is approximately the same as meters water equivalent (m.w.e.). . . . 23

3.2 Surface muon angular distribution in comparison with the distribution proposed by Miyake [13] at 265 m.w.e. . . . . . . . . . . . . . . . . . . 25

3.3 World data for neutron production in scintillator. The curve was proposed by Agafonova and Malgin [2], Eqn. 3.3. See Table 3.1 for references and identification of which results have been corrected by Agafonova and Malgin. 26

3.4 The differential energy spectrum for muon-induced neutrons at various underground sites. The plot was made by Mei and Hime [14]. . . . . . . . . . . 29

4.1 Feynman diagrams for the Charge Current Quasi-Elastic (CCQE) and Neutral Current elastic (NCel) neutrino interactions whose cross sections FINeSSE was designed to measure. . . . . . . . . . . . . . . . . . . 31

4.2 Schematic showing the SciBath event detection principle for charged particle detection. For a neutral particle to be detected it must interact in the scintillator and create charged particle tracks. . . . . . . . . . . . . . . 32

4.3 Schematic and photographic overviews of the the SciBath Detector. The SciBath detector cube and optical fibers are usually covered by protective panels, but those were removed for this photograph. The LED calibration system (green boards) can be seen as well as the optical fibers that guide their light to WLS fibers in the cube. . . . . . . . . . . . . . . . . . . 33

4.4 Schematics of the WLS fiber grid and the SciBath detector cube. The lower front corners of the cube walls have been removed, exposing the WLS fibers

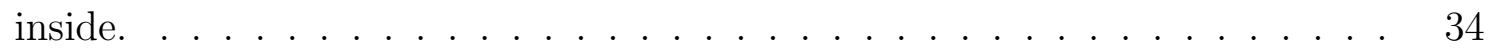

4.5 The SciBath detector, data acquisition system, and supporting equipment loaded in a moving truck for deployment at FNAL.

4.6 a) Emission spectrum for EJ scintillator (similar to ours), b) absorption (dotted) and emission (solid) spectra of our (UB) WLS fibers, c) quantum efficiency of our PMTs. $\ldots \ldots \ldots \ldots$. . . . . . . . . . . . . . . . 36

4.7 The SciBath detector with its data acquisition system complete for datataking. Pictured from left to right is the SciBath detector, a half rack with power supplies and trigger logic, and the data acquisition computer. . . . . . 38

4.8 Hamamatsu 64 anode photomultiplier tube (face down, left) next to its voltage divider "base" board (right), designed at Indiana University. . . . . . . . . . 39 
4.9 An integrated readout module (IRM). SciBath uses 12 IRMs to read out the detector and perform low level analysis and buffering. Each IRM reads out 64 wavelength-shifting fibers and has an integrated 64-anode photomultiplier tube (shown on the right side of the board). . . . . . . . . . . . . . . 40

4.10 A PMT mounted to an IRM. The PMT housing ensures proper alignment with the corresponding readout fiber bundles. . . . . . . . . . . . . . . 40

4.11 Two VME crate "shells" that hold the integrated readout modules on the SciBath detector and and align their PMTs with readout fiber bundle cookies (the black rectangles). Also pictured is a board (lower left) that regulates power and clock synchronization signals to the integrated readout modules through a ribbon cable. . . . . . . . . . . . . . . . . . . 41

4.12 VME crate "shells" filled with the full set of readout electronics, complete for data-taking. Cables shown include high voltage to photomultiplier tubes (red) and ethernet cables for data transfer (orange). . . . . . . . . . . . . . . 41

4.13 Two sample photomultiplier tube waveforms in SciBath. The standard photomultiplier tube signals have been modified by "ringing oscillator" shaping

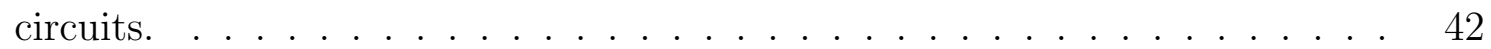

4.14 Schematic of the LED calibration system showing from left to right: pulsed LED system, LED end optical fibers, WLS fibers inside the detector cube, readout end optical fibers, and a PMT. . . . . . . . . . . . . . . . . . . 44

4.15 LED calibration system boards and their corresponding optical fibers. . . . . 45

4.16 Schematic of the the SciBath nitrogen and liquid scintillator plumbing systems. 46

4.17 The view from one of two webcams with which SciBath can be monitored remotely. Two external leak containers can be seen underneath the detector (one yellow, one black). . . . . . . . . . . . . . . . . . 50

4.18 The view from one of two webcams with which SciBath can be monitored remotely. The two gauges at the top center give the internal pressure; the three gauges at the right give the $\mathrm{N}_{2}$ flow rates; the white gauge to the left gives the time, temperature, and humidity; and the digital gauge at the bottom right gives the scintillator level in the reservoir tank. . . . . . . . . . . . . . 50

4.19 The SciBath run control GUI, which also gives the run status during monitoring. 51

4.20 This diagnostic output for real time run monitoring provides run summary statistics including event rates (top and left), error reports (right), and fiberby-fiber response summaries (bottom). . . . . . . . . . . . . . 52

4.21 This run summary diagnostic provides PE, multiplicity, event count, and waveform baseline summaries. . . . . . . . . . . . . . . . . . . . 53

5.1 Side view of the NuMI beam line at FNAL. On the left side is the iconic Wilson Hall and on the right side is the MINOS hall, where SciBath was deployed. The dotted line represents the NuMI beamline. . . . . . . . . . . . 55

5.2 The SciBath detector being lowered to the MINOS near detector hall at FNAL. 55

5.3 Cumulative protons on target (POT) versus time during the NuMI run. . . . 58 
6.1 Examples of waveform fitting. The red curve shows a fit with Eqn. 6.1. The green line indicates the fitted start time and the blue point indicates the minimum of the pulse, which was used to calculate the pulse amplitude. . . . 62

6.2 Histograms of the number of LED pulses versus hit charge as measured in analog-to-digital converter channels. The curve is a Gaussian-smeared Poisson function with Poisson mean "mu" in photoelectrons (PEs) and Gaussian sigma equal to "sigscale" times the number of PEs. The peaks at 40 ADC channels are the one-PE response. A potential two-PE response is barely visible in the lower plot at 75 ADC channels. . . . . . . . . . . . . . . . . . 64

6.3 Diagram of the event building process. The first hit opens a $300 \mathrm{~ns}$ time window and all hits within are grouped into the first event. The first hit to occur after this window opens its own time window for the second event, and the process repeats. Event 1 is likely caused by PMT noise. Event 2 represents a typical event in SciBath. Events 3 and 4 show how a random PMT noise hit preceding a particle interaction can split the resulting hits into two separate events. The PMT noise rate is such that event splitting is rare; it is shown here simply to illustrate the event grouping method. . . . . . . . . . . . . . 65

6.4 Event energy versus event multiplicity for $1 / 40$ of the NuMI data set. The linearity indicates a strong correlation of roughly 2 PEs per PMT hit, on

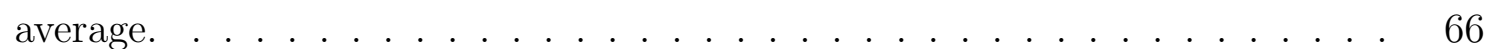

6.5 Timing analysis for cosmogenic neutron capture correlated events. An event well after any NuMI beam pulse that passes energy and topology cuts was considered a cosmic neutron or muon primary candidate and opened a $200 \mu \mathrm{s}$ window to search for neutron capture candidate events. . . . . . . . . . . . . 67

6.6 Timing analysis for beam-correlated neutron events. An event during a NuMI beam pulse that passes energy and topology cuts must be followed by a neutron capture candidate in a $450 \mu \mathrm{s}$ window ("n capture") that starts $5 \mu$ s after the beam pulse. . . . . . . . . . . . . . . . . . 68

6.7 Distribution of average hit time within an event for all cosmic data that deposited more than 12.5 PEs of energy. Neutron capture event selection required average time less than $40 \mathrm{~ns}$. . . . . . . . . . . . . . . . . 69

6.8 Distribution of hit time standard deviation within an event for all cosmic data that deposited more than 12.5 PEs of energy. Neutron scatter and muon event selection required $\sigma$ less than 100 ns while neutron capture event selection required $\sigma$ less than $40 \mathrm{~ns}$. . . . . . . . . . . . . . . . . . . . . . . . . . . . . 69

6.9 Photoelectron spectrum for all events in the NuMI beam. Minimum ionizing muons are responsible for the peak at 400 PEs. . . . . . . . . . . . . 70

6.10 Sample SciBath event displays. The image at the top center shows the SciBath coordinate system. The plot on the lower right shows the hit time distribution within the event. Basic event and analysis summary information is included at the top right. . . . . . . . . . . . . . . . . . . 73

6.11 This ellipsoid is similar in shape to the energy deposition of a minimum ionizing particle in SciBath. . . . . . . . . . . . . . . . 76 
6.12 Largest eigenvalue versus photoelectron response for beam uncorrelated (correlated) MI-12 events, which are primarily muons (neutrons) between 250 and 550 photoelectrons. These plots are count normalized in photoelectron response bins to clearly show the structure at higher photoelectron responses, which contain fewer events. . . . . . . . . . . . . . . . . . 77

6.13 Fit of the time difference between neutron scatter and capture candidate events. The time range is much larger than the 163 us average time difference to allow an accurate fit of the accidental background rate for neutron capture candidates. . . . . . . . . . . . . . . . . . . . . . . . . 79

6.14 Geant4 simulation of a $19.4 \mathrm{GeV}$ muon in the SciBath detector. The muon track is red and the scintillation photon tracks are green. The number of scintillation photons was reduced by a factor of 3 for this event to enable details to be seen. . . . . . . . . . . . . . . . . . . . . . 85

6.15 Geant4 simulated photoelectron response for neutron scattering events versus incident neutron energy. Requiring neutron capture ensures the photoelectron response is much more correlated to the incident neutron energy. . . . . . . . 88

6.16 External neutron capture efficiency for neutrons entering SciBath as determined by simulation of neutrons generated uniformly on a sphere surrounding the detector. Detection of the $2.2 \mathrm{MeV}$ capture photon is not included in this

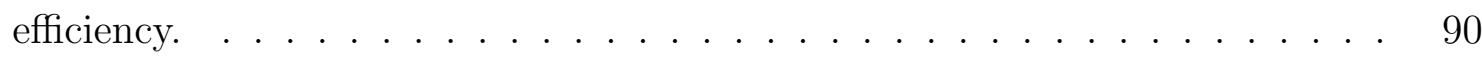

6.17 Neutron capture efficiency for SciBath for internally generated neutrons, as determined by a simulation of neutrons generated uniformly in the detector. Detection of the $2.2 \mathrm{MeV}$ capture photon is not included in this efficiency. . $\quad 93$

6.18 Neutron capture efficiency for SciBath for internally generated neutrons, as determined by a simulation of neutrons generated uniformly in the detector. The efficiency for detection of the $2.2 \mathrm{MeV}$ capture photon is not included in this efficiency. . . . . . . . . . . . . . . . . . . . . 94

6.19 Simulated cosmic muon track length distributions. . . . . . . . . . . . . . . . 96

6.20 Simulated muon reconstructed distance of average energy response from the detector center. . . . . . . . . . . . . . .

7.1 Muon fluxes versus effective fiducial cross sectional area for five different methods. The lines drawn simply connect the data points to guide the eye. The "Sphere 2" at $791 \mathrm{~cm}^{2}$ was used for the muon flux result. The drop and divergence in fluxes at small fiducial volumes is understood to be caused by reductions in the SciBath effiective cross sectional area for imperfectly simulated high angle muons, which varies with the fiducial method. . . . . . . . . 102

$7.2 \quad$ Geant4 simulated detector energy response to $2.2 \mathrm{MeV}$ photons. . . . . . . . 104

7.3 Beam correlated 2.2 MeV photon response with the largest reasonable Gaussian fit. The discrepancy between the data and the fit at low PE is due to the soft threshold caused by our particle identification cuts, primarily the multiplicity greater than five requirement. . . . . . . . . . . . . . . . . . 104

7.4 Angular distribution of muons at $100 \mathrm{~m}$ underground at the MINOS hall at

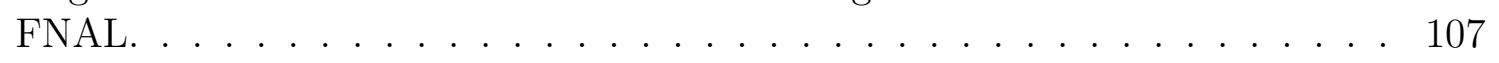


7.5 Azimuthal angular distribution of muons at $100 \mathrm{~m}$ underground at the MINOS hall at FNAL. The angle $\theta$ is measured from the vertical. The curve drawn is the prediction by Miyake [13]. . . . . . . . . . . . . . . . . . . . . 108

7.6 World data for neutron production in scintillator, including our result. The curve was proposed by Agafonova and Malgin and is reproduced in Eqn. 3.3. See Table|3.11 for references and identification of which results have been corrected by Agafonova and Malgin.

7.7 Distribution of the time between neutron primary (scatter) and secondary (capture) events. The curve is a decaying exponential of characteristic time $\tau=163 \mu \mathrm{s}$, which is the time structure we expect for neutron capture in SciBath. The distribution is normalized to the number of neutrons counted. 118

7.8 Neutron energy spectrum at $100 \mathrm{~m}$ underground at the MINOS hall at FNAL, normalized to the measured flux. The prediction is an extrapolation of the results of Mei and Hime [14]. The errors shown are the statistical errors associated with the background subtracted neutron count, $N_{n, n}$. The systematic

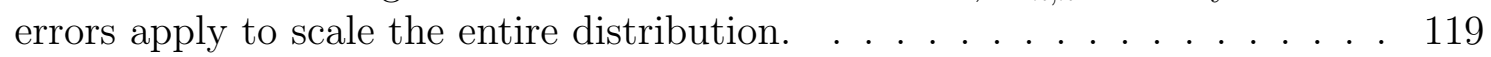

7.9 Neutron energy distribution normalized to the ratio of the measured neutron and muon fluxes. The prediction is an extrapolation of the results of Mei and Hime [14]. The errors shown are the statistical errors associated with the background subtracted neutron count, $N_{n, n}$. The systematic errors apply to scale the entire distribution. . . . . . . . . . . . . . . . . . . . . . . 119

7.10 The SciBath integrated detector response before, during, and after the NuMI beam pulse. . . . . . . . . . . . . . . . . . 120

7.11 Event rate before, during, and after the NuMI beam pulse for muon-, neutron scatter-, and neutron capture-like events. The neutron scatter and neutron capture candidate events are fit with decaying exponentials after the beam pulse. . . . . . . . . . . . . . . . . . . . . . . . . . . . 121

7.12 Angular distribution of NuMI beam correlated muons at the MINOS hall at FNAL. The angle in the right plot is measured from the beam direction. . . 122

7.13 Background subtracted distribution of the time between the beam center and following neutron capture candidate events. The curve is the fitted decaying exponential that resulted in the $\tau=163 \mu$ s characteristic time used in the cosmic analysis. . . . . . . . . . . . . . . . . . . 122

7.14 Background subtracted distribution of the time between neutron primary (scatter) and secondary (capture) beam correlated events. The curve is a decaying exponential of characteristic time $\tau=163 \mu \mathrm{s}$, which is the time structure we expect for neutron capture in SciBath. . . . . . . . . . . . . . . 123

7.15 Neutron energy spectrum correlated to the NuMI beam at the MINOS hall at FNAL. The energy calibration includes the proton quenching analysis of Sec. 6.3 .2 , The errors are statistical and result from the background subtraction. 124

7.16 Neutron capture energy spectrum correlated to the NuMI beam at the MINOS hall at FNAL. The errors are statistical and result from the background

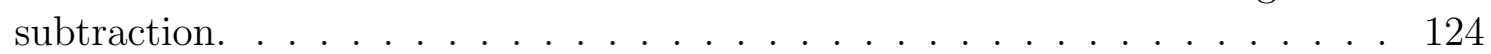

7.17 Left: double beta decay; right: neutrinoless double beta decay. . . . . . . . . 125 
7.18 Multi-site (MS, top) and single-site (SS, bottom) energy spectra. The bestfit line (solid blue) is shown. The background components are $2 \nu \beta \beta$ (grey region), ${ }^{40} \mathrm{~K}$ (dotted orange), ${ }^{60} \mathrm{Co}$ (dotted dark blue), ${ }^{222} \mathrm{Rn}$ in the cryostatlead air-gap (long-dashed green), ${ }^{238} \mathrm{U}$ in the TPC vessel (dotted black), ${ }^{232} \mathrm{Th}$ in the TPC vessel (dotted magenta), ${ }^{214} \mathrm{Bi}$ on the cathode (long-dashed cyan), ${ }^{222} \mathrm{Rn}$ outside of the field cage (dotted dark cyan), ${ }^{222} \mathrm{Rn}$ in active xenon (long-dashed brown), ${ }^{135} \mathrm{Xe}$ (long-dashed blue) and ${ }^{54} \mathrm{Mn}$ (dotted brown). The ${ }^{137} \mathrm{Xe}$ background component is not shown, but includes the entire energy range. The $0 \nu \beta \beta$ region of interest is at $2.458 \mathrm{MeV}$. Figure from the EXO Collaboration [15].] . . . . . . . . . . . . . . . . . . . . . . . . . . . . 127

7.19 Muon energy distributions at NuMI and WIPP. Curves from Eqn. 8 of Ref. [14] with the parameters suggested by Groom et al. [1] and Battistoni et al. [16]]. 128

8.1 Event display for a neutron double-scattering candidate event. . . . . . . . . 131

8.2 Point-like versus track-like characterization parameters for a primarily muon data set (upper left) and a primarily neutron data set (lower right), both from the MI-12 run. . . . . . . . . . . . . . . . . . . . 131 


\section{Chapter 1}

\section{Introduction}

This thesis presents the SciBath detector and five measurements it conducted of muon and neutron characteristics at $100 \mathrm{~m}$ underground: the muon flux, the muon angular distribution, the neutron yield in scintillator from muon spallation, the neutron flux, and the neutron energy spectrum.

SciBath is a $80 \mathrm{~L}$ liquid scintillator detector that was designed for neutral particle detection. The cubic liquid scintillator "bath" houses a three dimensional grid of 768 wavelengthshifting (WLS) fibers with $2.54 \mathrm{~cm}$ fiber spacing. This allows for a detailed reconstruction of charged particle tracks, including those that result from neutral particle interactions in the scintillator volume. Originally designed to study neutrino interactions in this way, it is also a good neutron detector as this thesis presents. The detector assembly and commissioning was finished in the fall of 2011 before its first production data run in the same season. This run was dubbed the "NuMI run" after the NuMI beam line at the Fermi National Accelerator Laboratory (FNAL) where the detector conducted its measurements.

Cosmic ray muon-induced fast neutrons are an important background for sensitive un-

derground experiments. Neutrons can be a background to solar neutrino and low-energy neutrino oscillation experiments because they can imitate neutrino detection in scintillator through inverse beta decay [17, 18, 19]. As a specific example, fast neutrons were found to 
be the dominant background for the Palo Verde reactor neutrino oscillation experiment [17]. Some dark matter experiments are concerned about neutron-proton elastic scattering creating nuclear recoils that mimic the Weakly Interacting Massive Particle (WIMP) signal, e.g. the CDMS experiment [20]. Through inelastic scattering and radiative capture, neutrons can contribute to the $\gamma$-ray background of double beta decay experiments [15]. These neutrons have energies up to the $\mathrm{GeV}$ scale making them impractical to passively shield. In fact, many shielding materials will act as a target for further neutron production and actually increase the neutron background! Furthermore, since these neutrons are can travel many meters from their production site, not all of them can be removed by an active veto system.

Muons themselves are not an important background to most underground experiments because they are generally easy to identify and veto, but their fluxes are important to understand because fast neutrons more than $10 \mathrm{~m}$ underground are exclusively produced through cosmic ray muon interactions. The muon flux at depth has been studied by a number of authors at varied sites since the 1950s [12]. These muons are generated in the atmosphere through cosmic ray showers and penetrate to the deepest underground experiment locations. Despite a relatively well accepted parameterization for the energy loss of a muon passing through matter, precise predictions of the flux at a particular site are difficult primarily because of the complications of rock composition and overburden geometry (mountainous, flat, etc.).

Fast neutrons underground can be categorized by whether they are produced in the earth or detector materials. Characteristics of those produced in the earth are largely unstudied by measurement or simulation. Characteristics of those produced in detector materials have been studied by a number of measurements and simulations at various depths [2, 14], but the results are often mutually inconsistent.

The muon flux and angular distribution measurements presented in this thesis contribute to the understanding of muons underground. In similar fashion, it is hoped the neutron flux and yield measurements presented here are equally useful. However, the neutron measure- 
ments have fairly large errors and may prove to be most useful as a guide future work with similar goals.

\subsection{Structure of this thesis}

This thesis begins in Chapter 2 with a review of the science and relevant interactions of underground muons and neutrons. Chapter 3 summarizes the previous measurements and simulations of muon and neutron characteristics underground, with which our results can be compared. Chapter 4 describes the SciBath detector, including its principle of operation, mechanical aspects, and data acquisition system (DAQ). Chapter 5 provides a summary of the NuMI run, including its timeline and location. Chapter 6 describes the analysis methodology that led to our results. Chapter 7 presents the results of our measurements of neutron and muon characteristics at the NuMI site as well as comparisons with the measurements described in Chapter 3. Finally, Chapter 8 offers a concluding summary of the information presented in this thesis. 


\section{Chapter 2}

\section{Muons and Neutrons Underground}

In this thesis I present results of our measurement of neutron and muon characteristics at $100 \mathrm{~m}$ underground. Those results and the analyses that led to them are described in later chapters. In this chapter I first provide a summary of the processes that determine neutron and muon characteristics underground. I also describe the mechanisms by which these particles interact with the SciBath detector and, therefore, the signatures for their identification.

\subsection{Muons underground}

Underground muons have two sources: neutrino interactions in the earth and cosmic ray showers in the upper atmosphere. The former only significantly contribute to the total flux for depths greater than $10 \mathrm{~km}$.w.e. and are thus beyond the scope of this thesis. The latter are dominant for all shallower depths and are the ultimate source of neutrons measured in this analysis.

The unit km.w.e. stands for "kilometers water equivalent" and is more universal for the description of depth because a water overburden does not have the composition or surface geometry complications that rock does. One kilometer of standard rock with a flat overburden is equivalent to $2.65 \mathrm{~km} . \mathrm{w} . \mathrm{e}$. 
Cosmic rays are extraterrestrial, high-energy particles incident on the upper atmosphere of the earth. They are composed of a variety of nuclei as well as electrons, protons, and positrons. Their energy distribution is peaked around $0.3 \mathrm{GeV}$ but extends above $10^{9} \mathrm{GeV}$, the regime of ultra-high-energy cosmic rays. With the exception of those created in solar flares they are generated outside of the solar system. Cosmic ray interactions in the upper atmosphere generate complex particle showers, from which only neutrinos and muons penetrate more than a few meters into the surface of the earth.

Muons, like all charged particles, lose energy as they pass through matter. The loss mechanisms include ionization and radiative processes: photonuclear interactions, $e^{+} e^{-}$pair production, and bremsstrahlung. The resulting energy loss takes the well accepted form

$$
-\frac{d E_{\mu}}{d X}=a+b E_{\mu}
$$

where $a$ is the ionization loss and $b$ is radiative loss, both of which are energy dependent. Integration gives the muon energy as a function of depth and initial energy, $E_{\mu, 0}$ :

$$
E_{\mu}\left(E_{\mu, 0}, X\right)=\left(E_{\mu, 0}+\frac{a}{b}\right) \exp (-b X)-\frac{a}{b}
$$

This expression can be used with the parameters calculated for standard rock by Groom et al. [1] to determine the range of muons in the earth, including those plotted in Table 2.1. Interestingly, even though all muons lose energy as they penetrate the earth, the mean muon energy increases as a function of depth as described in Eqn. 3.7. This is because the muon energy spectrum at the surface is concave up and strongly peaked at the lowest energies. As depth increases the lower energy muons are stopped and the resulting energy distribution is less peaked, resulting in a higher average energy. 


\begin{tabular}{|c|c|c|c|}
\hline$E_{\mu, 0}(\mathrm{MeV})$ & $a\left(\mathrm{MeV} \mathrm{g}^{-1} \mathrm{~cm}^{2}\right)$ & $b\left(10^{-6} \mathrm{~g}^{-1} \mathrm{~cm}^{2}\right)$ & range (km.w.e.) \\
\hline$\overline{10}$ & 2.17 & $\bar{~} 1.90$ & 0.05 \\
\hline 100 & 2.44 & 3.04 & 0.41 \\
\hline 1000 & 2.68 & 3.92 & 2.45 \\
\hline 10000 & 2.93 & 4.35 & 6.09 \\
\hline
\end{tabular}

Table 2.1: Parameters for Eqn. 2.2 as calculated by Groom et al. [1] and resulting muon ranges in standard rock

\subsection{Muons in SciBath}

Muons are among the easiest particles to detect in liquid scintillator detectors. As charged particles with very long attenuation lengths, they usually create long, energetic tracks. This section describes the concept of a minimum ionizing muon, which was used for the energy calibration of SciBath; and the process of muon decay, which could act as a background for our neutron flux measurement.

\subsubsection{Minimum ionizing muons}

Muons passing through matter can interact in many ways including:

1. inelastic collisions with electrons,

2. elastic scattering with nuclei,

3. nuclear reactions,

4. emission of Cherenkov radiation, and

5. bremsstrahlung.

The first two of these are by far the most common, and inelastic collisions with electrons are almost entirely responsible for the energy loss of charged particles heavier than the electron [21]. The average amount of energy lost as a particle penetrates a unit length of a material is called its stopping power, $d E / d x$. Stopping power depends on the particle type, the particle energy, the material type, and the material density. Figure 2.1 shows 
the stopping power for a muon in copper as a function of muon kinetic energy. At about $100 \mathrm{MeV}$ the stopping power is minimum and the muon is called minimum ionizing. For the next three orders of magnitude in muon energy the muon stopping power remains relatively constant. This feature was used in the energy calibration described in Sec. 6.3.1.

The Scibath target is liquid scintillator, not copper. However, the stopping power for heavy particles in matter is almost independent of material type. Following the presentation by Leo [21], the stopping power curve in liquid scintillator is expected to agree with that for Copper to within $10 \%$ through the minimum ionizing energy range.

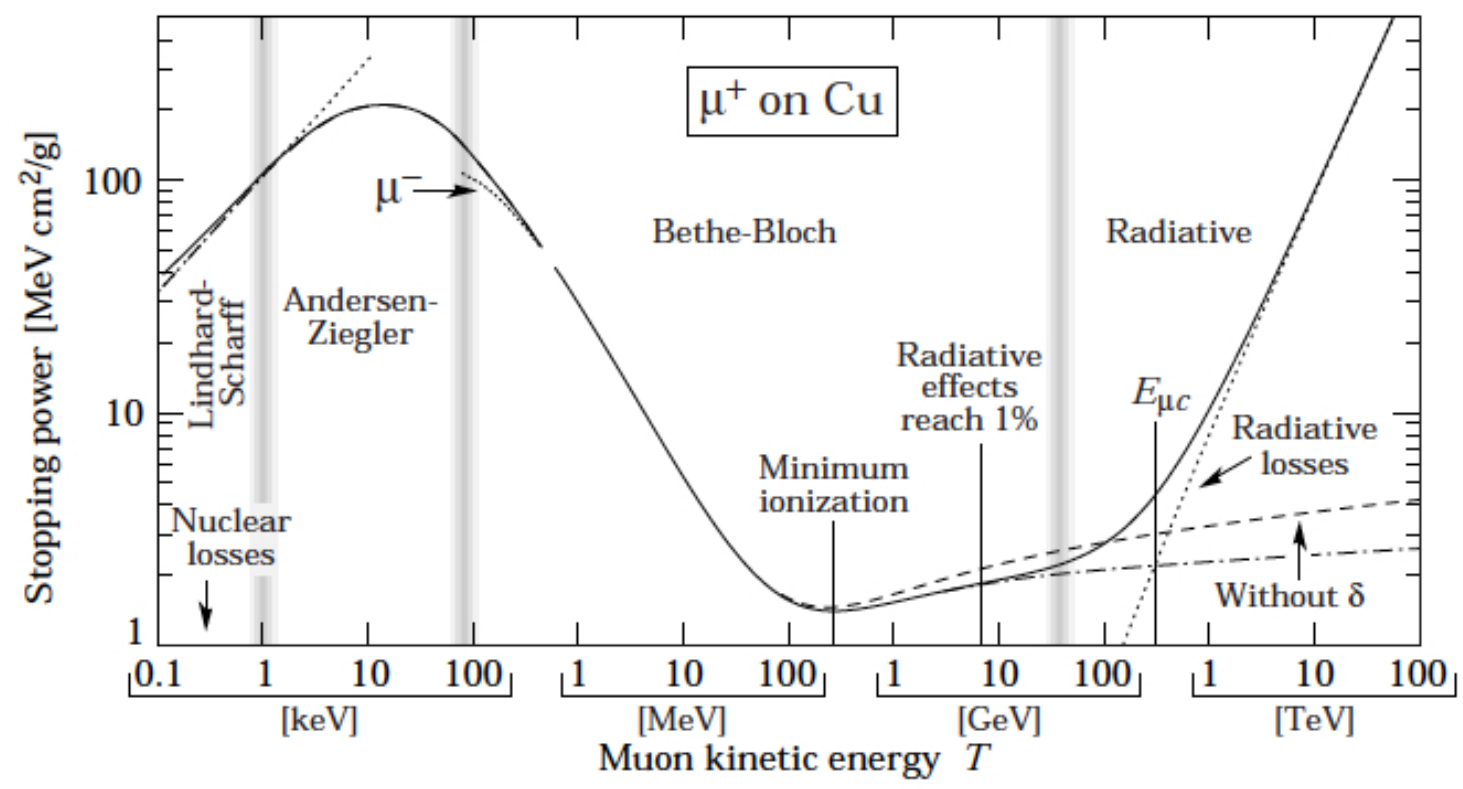

Figure 2.1: Stopping power $(\langle-d E / d x\rangle)$ for positive muons in copper as a function of kinetic energy, $T$, over 12 orders of magnitude. Solid curves indicate the total stopping power. Data below the break at $T \approx 0.5 \mathrm{MeV}$ are taken from ICRU 49 [3], and data at higher energies are from Ref. [1]. Vertical bands indicate boundaries between different theoretical approximations or dominant physical processes. The short dotted lines labeled " $\mu$ " illustrate the "Barkas effect," the dependence of stopping power on projectile charge at very low energies [4, 5]. "Nuclear losses" indicates non-ionizing nuclear recoil energy losses. Figure from Groom et al. [1]. 


\subsubsection{Muon decays}

The muon is an unstable particle that decays through the weak interaction with a mean lifetime of $2.2 \mu \mathrm{s}$ in vacuum. The dominant decay, often called Michel decay, is also the simplest decay mode:

$$
\begin{aligned}
& \mu^{-} \rightarrow e^{-}+\bar{\nu}_{e}+\nu_{\mu}, \\
& \mu^{+} \rightarrow e^{+}+\nu_{e}+\bar{\nu}_{\mu} .
\end{aligned}
$$

An "in-flight" muon (or antimuon) decay in SciBath could consist of a single event with two particle tracks: a relatively long track for the incident muon and a very short track for the electron. Because the decay is nearly instantaneous, both of these tracks would be grouped into a single event in SciBath. Alternatively, a muon can be captured by the Coulomb field of a nucleus and form a "muonic atom." If this muon later undergoes Michel decay the muon track and electron tracks can create two events in SciBath separated by a few microseconds. To avoid misidentifying these coincidence events as neutrons this analysis requires at least 5 us between neutron scatter and neutron capture candidate events. Of course, antimuons are positively charged and cannot be captured. Thus, antimuon decays in SciBath are all "in-flight" decays and cannot contaminate a coincidence measurement. Muon capture is also relevant for neutron production and is further described in Sec. 2.3.4.

\subsection{Neutron sources underground}

Neutrons can be produced underground through a variety of processes. This section describes the four dominant processes that are relevant to underground experiments: neutrons from the hadronic component of cosmic ray showers, from natural radioactivity in the earth, from cosmic ray muon-induced spallation, and from muon capture. Fig. 2.2 gives an overview of the flux contributions of these processes as a function of depth. 


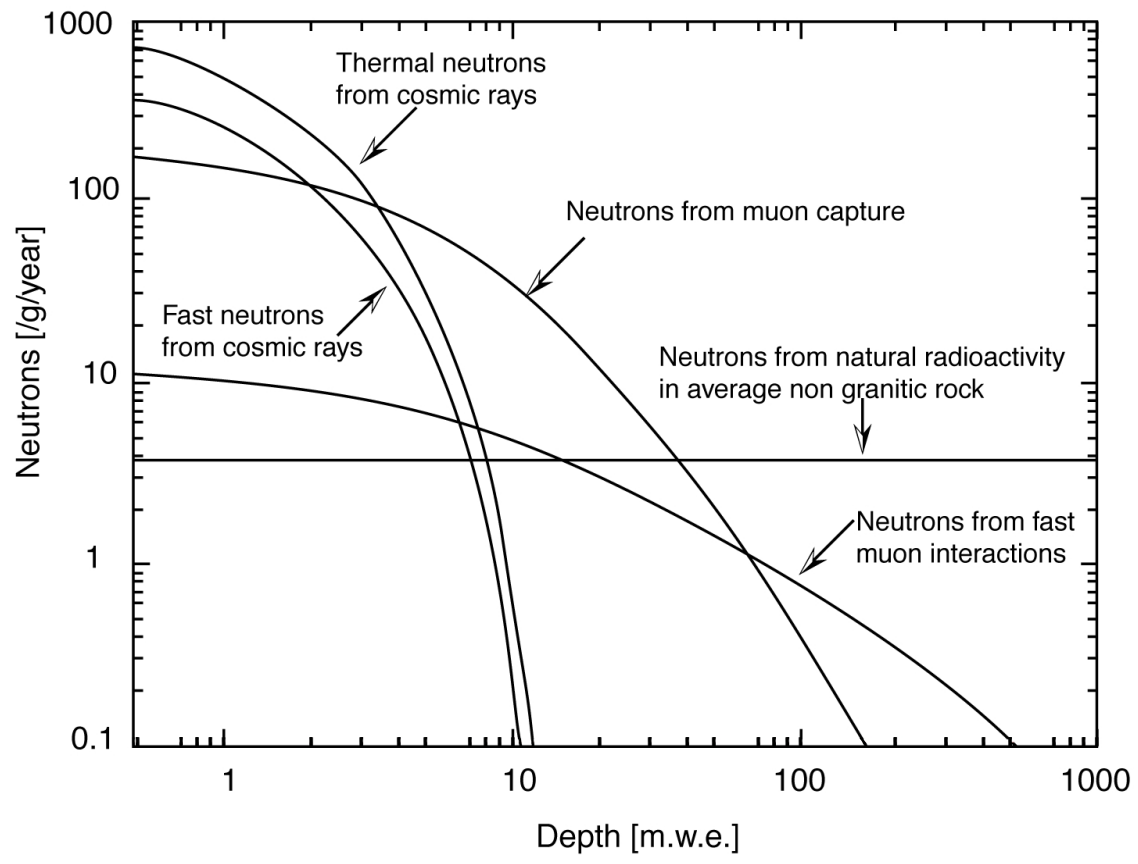

Figure 2.2: Total neutron fluxes for different sources as a function of depth in meters water equivalent. At NuMI depth only neutrons from muons contribute to the flux above $16 \mathrm{MeV}$. Figure adapted from the Ph.D. thesis of E.-I. Esch [6].

\subsubsection{Hadronic component of cosmic rays}

Cosmic rays are high-energy charged particles, usually protons or atomic nuclei, that strike the earth from astronomical sources. These particles interact in the upper atmosphere and generate showers of secondary particles that reach the surface of the earth. Two components of these showers of particular note for this analysis are muons and neutrons. Cosmogenic muons can penetrate to the deepest underground laboratories and interact with the rock or detector materials to generate their own secondary particles, see Sec. 2.3.3. Neutrons from the hadronic component of cosmic rays can penetrate the surface of the earth and represent the dominant source of neutrons at shallow depths. As Fig. 2.2 shows, however, these neutrons are insignificant compared to neutrons from muons or natural radioactivity at depths greater than 10 m.w.e. During the NuMI run SciBath was located 100 m, 265 m.w.e., underground and well beyond the reach of neutrons from the hadronic component of cosmic rays. 


\subsubsection{Radioactivity in the earth}

Uranium, thorium, and other heavy elements can be found in the earth at all depths. These heavy elements can produce neutrons by two processes: fission and $\alpha$ decay. The $\alpha$ decay itself does not emit neutrons, but an $\alpha$ particle can interact with a nucleus to create a neutron in what is known as an $(\alpha, \mathrm{n})$ reaction. The energy of the $\alpha$ particle depends on the details of the radioactive decay but is generally around $5 \mathrm{MeV}$. The energy of the outgoing $(\alpha, \mathrm{n})$ neutron depends on the target nucleus, but is at most $10.8 \mathrm{MeV}$ from the interaction with ${ }^{9} \mathrm{Be}[22]$.

The energy of fission neutrons can be characterized by a Maxwellian distribution with an average energy depending on the particular nucleus, but generally around $2 \mathrm{MeV}$ [23, 24]. The flux of neutrons from these radioactive processes in standard rock is shown in Fig. 2.2, and at the NuMI run depth of 265 m.w.e. they are the dominant source of neutrons. However, because the minimum neutron energy for this measurement, $16 \mathrm{MeV}$, is well above the energies of neutrons from natural radioactivity, they have no effect on the analysis.

\subsubsection{Neutron production through muon spallation}

Nuclear spallation is the process by which a high energy particle strikes a nucleus causing the ejection of a number of nucleons. Cosmic ray muons induce spallation in the earth and detector materials at all depths. Figure 2.2 shows relative flux versus depth and Figure 3.4 shows simulated energy spectra for the resulting cosmogenic neutrons at various sites. While most of the neutrons are low in energy, high energy neutrons are also created. These higher energy neutrons are important to characterize because they are impractical to completely shield. In fact, attempts to shield them may actually induce a larger background as the shielding material acts as a target for further spallation. The characterization of these neutrons from spallation in the earth is the primary goal of this analysis. A schematic of the detection signal for this production process in the SciBath liquid scintillator is shown in Fig. 2.3 . 


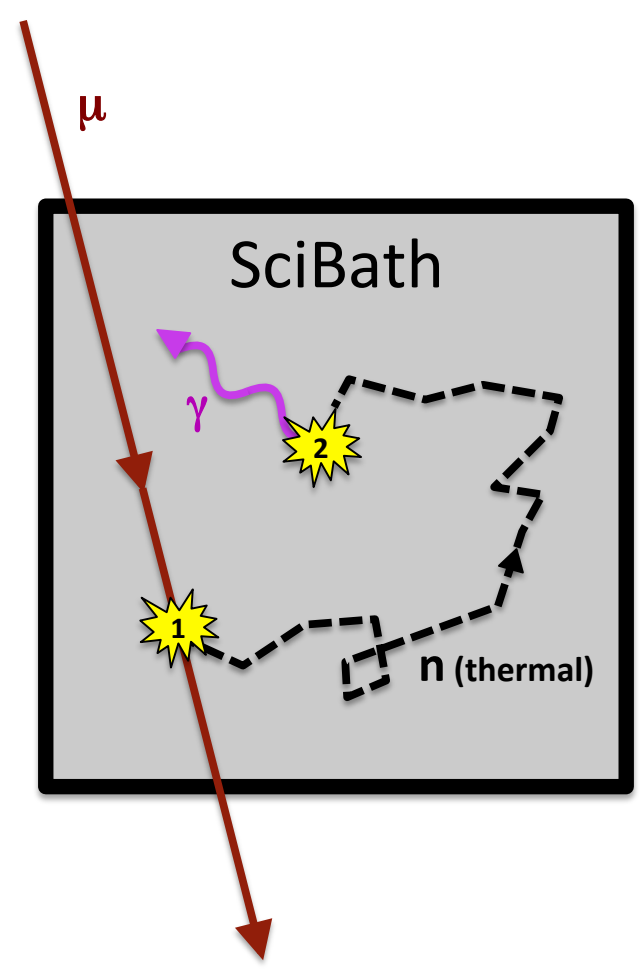

Figure 2.3: Schematic of neutron production through muon spallation inside the detector. SciBath detects the muon followed by a $2.2 \mathrm{MeV}$ photon at a characteristic time near 160 us. 


\subsubsection{Muon capture}

Section 2.2 .2 described how muons can capture in liquid scintillator and then Michel decay according to Eqn 2.3. The muon interaction with a proton,

$$
\mu^{-}+\mathrm{p} \rightarrow n+\nu_{\mu}
$$

is a less common result of muon capture, but acts as a source of neutrons both inside and outside of a detector.

As indicated in Fig. 2.2, neutrons produced through muon capture in the rock may be responsible for a detectable fraction of the neutron flux present at $100 \mathrm{~m}$ (265 m.w.e.). Singer [25] gives the energy distribution of these neutrons as

$$
\frac{d N(E)}{d E} \approx \exp \left(-\frac{E}{E_{0}}\right),
$$

where $E_{0}$ has been measured at values between 7 and $15 \mathrm{MeV}$ for different materials [26, 27]. With this parameterization, at most $1 / 3$ of these neutrons are of energy greater than $16 \mathrm{MeV}$, so most would not be counted in our neutron flux measurement. Moreover, the majority of neutrons generated through muon capture share their energy with other nucleons in the capturing nucleus. In this case several low energy neutrons are "boiled" off of the nucleus in the process of nuclear evaporation. For these reasons neutrons generated through muon capture were judged to not significantly contribute to our neutron flux calculation.

However, neutrons produced through muon capture in the SciBath scintillator may contribute to the muon-induced neutron production measurement. Neutrons of all energies contribute to this measurement because a neutron scattering event is not required. In fact, lower energy neutrons are more likely to capture inside the detector. Figure 2.4 shows an expectation of the contribution to neutron production from muon capture. 


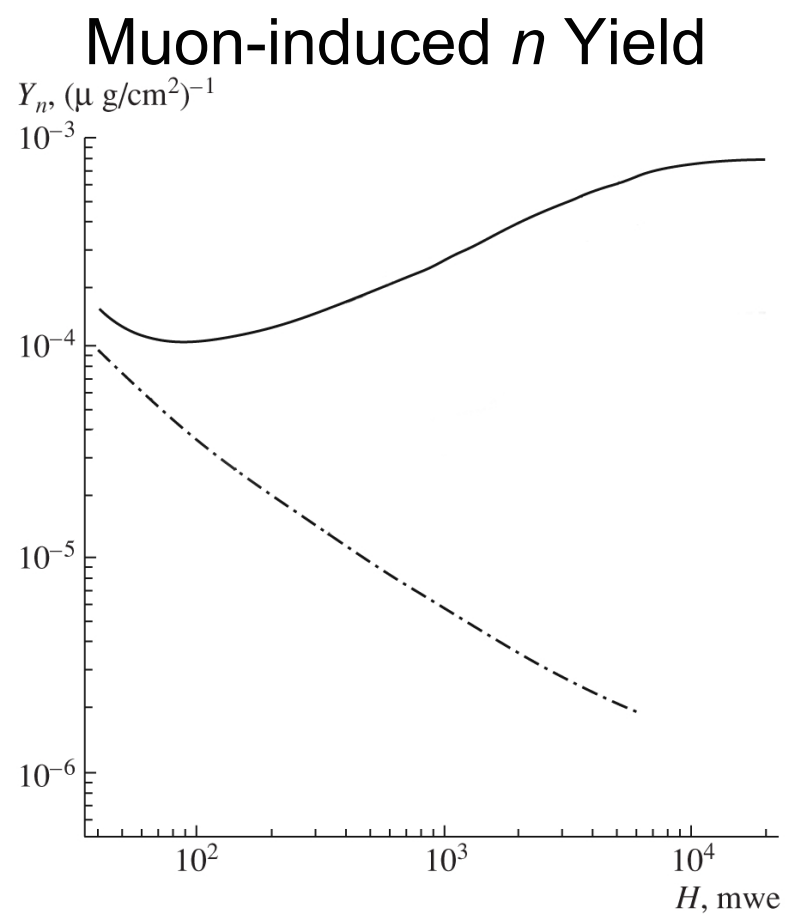

Figure 2.4: The expected total muon-induced neutron yield in liquid scintillator (solid) as a function of depth, and the contribution from muon capture (dotted). Figure adapted from Malgin and Ryazhskaya [7]. 


\subsection{Neutron interactions in SciBath}

Neutrons, like all neutral particles, cannot be detected directly in scintillator detectors. Instead, neutrons must interact with the target material in a way that results in a secondary particle that is charged and thus can induce scintillation. Neutrons are capable of interacting with matter in a large variety of ways. This section summarizes these possible interactions as relevant to SciBath and this analysis. In particular, the processes of elastic scattering off of hydrogen and radiative capture on hydrogen are discussed as well as a "typical" neutron timeline.

\subsubsection{Elastic scattering}

Elastic scattering can be thought of as a traditional "billiard ball" collision. The most common interaction a fast neutron will undergo in matter is elastic scattering with nuclei, primarily hydrogen and carbon in the case of liquid scintillator. Figure 2.5 shows the cross sections for neutron scattering and capture on hydrogen and carbon over a wide energy range. Some resonance structure is present in the carbon scattering cross section, but generally elastic scattering on carbon is more common above $10 \mathrm{MeV}$ and scattering on hydrogen is more common below. In liquid scintillator this dividing energy is actually a bit higher due to the 2:1 ratio of hydrogen:carbon atoms.

Elastic scattering on hydrogen is pictured in Fig. 2.6 and is much more significant than scattering on carbon for neutron detection because it produces a much more obvious scintillation signal. Since the mass of a hydrogen nucleus, i.e. a proton, is comparable to that of a neutron, elastic scattering mechanics favor a large transfer of energy between them: up to half the incident neutron energy. By contrast, a carbon nucleus is around 12 times as massive as a neutron, so relatively little of the neutron energy will be lost during an elastic scatter with carbon. Therefore elastic scattering with hydrogen nuclei creates a much higher energy recoil nucleus and a much more distinct scintillation signal. 


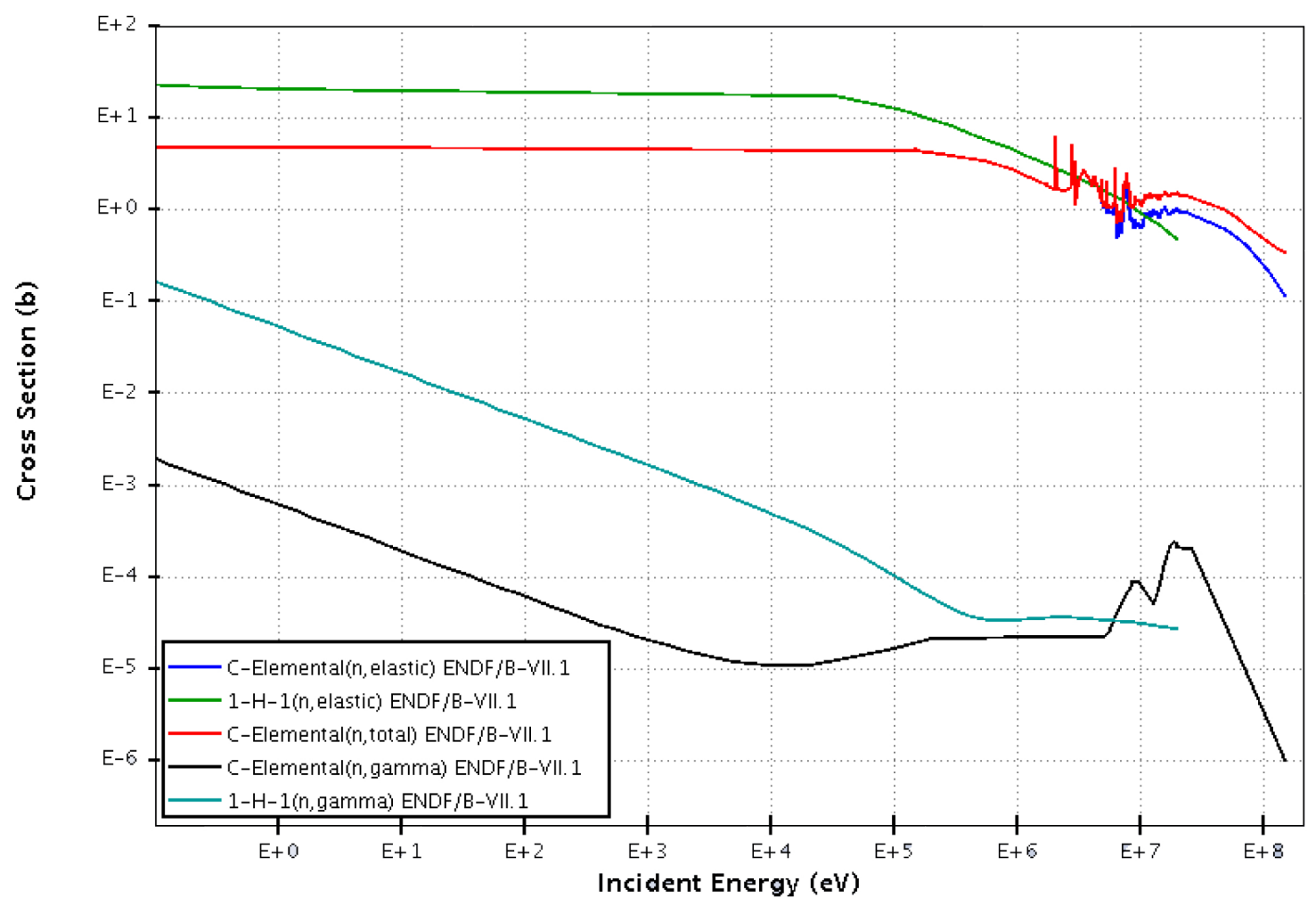

Figure 2.5: Cross sections for neutron interactions with hydrogen and carbon. The inelastic portion of the $\mathrm{n}-\mathrm{C}$ total cross section is only noticeable above $10 \mathrm{MeV}$. This data is from ENDF/B-VII.1 tables [8].

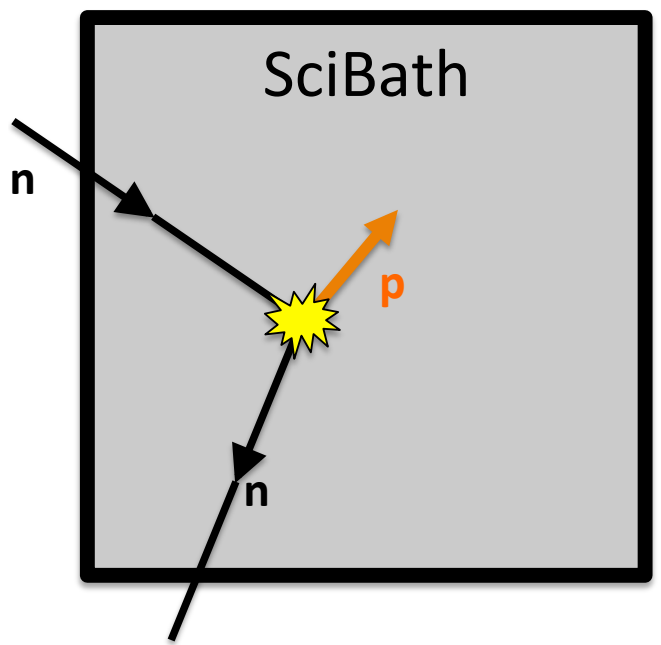

Figure 2.6: Schematic of neutron-proton elastic scattering event in SciBath, which is sensitive to the recoiling proton. 


\subsubsection{Radiative capture}

Radiative capture is the process by which a neutron is absorbed by a nucleus and a photon is released. The processes for hydrogen and carbon are

$$
\begin{aligned}
& n+{ }^{1} \mathrm{H} \rightarrow{ }^{2} \mathrm{H}+\gamma, \\
& n+{ }^{12} \mathrm{C} \rightarrow{ }^{13} \mathrm{C}+\gamma,
\end{aligned}
$$

and and their cross sections are shown in Fig. 2.5. Figure 2.7 summarizes the process of neutron capture in SciBath. The photon released in radiative capture is a characteristic 2.2 $\mathrm{MeV}$ in the case of hydrogen and $5.0 \mathrm{MeV}$ in the case of carbon. Either photon can be detected if it undergoes Compton scattering inside the detector.

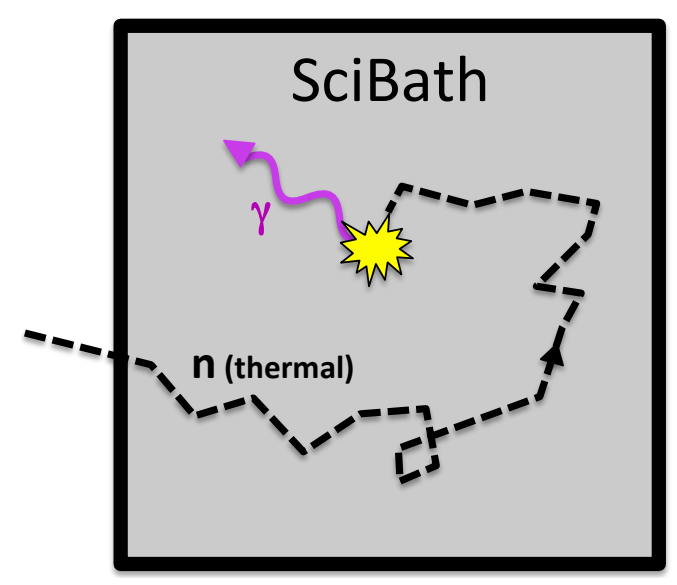

Figure 2.7: Neutron capture on a hydrogen nucleus in SciBath. SciBath is sensitive to the characteristic $2.2 \mathrm{MeV}$ photon.

An important concept in radiative neutron capture is that of a thermal neutron. This is a neutron of energy comparable to the thermal agitation energy of its surrounding atoms and molecules and is $\mathcal{O}(0.025) \mathrm{eV}$. A neutron at thermal energy is just as likely to gain energy as lose it in an elastic collision, so a thermal neutron tends to "rattle" around without losing energy until it undergoes radiative capture. 


\subsubsection{Inelastic scattering}

Inelastic scattering occurs similarly to elastic scattering except that the nucleus is left in an excited state. A minimum neutron energy is required to excite the nucleus, usually on the order of $1 \mathrm{MeV}$. In the case of carbon, elastic scattering occurs for energies above $5 \mathrm{MeV}$ as shown in Fig. 2.8. The figure also shows that above $20 \mathrm{MeV}$ the cross section for inelastic scattering on carbon is actually greater than that of elastic scattering on hydrogen. Thus a significant number of neutrons in liquid scintillator undergo inelastic scattering on carbon. The excited carbon nucleus has a variety of possible decay modes, some that produce scintillation signals and some that do not. The dominant inelastic processes are shown in Fig. 2.9. The neutron energy lost to neutron-carbon inelastic scattering interactions that do not produce a detector response contributes to a reduction in the neutron scatter detection efficiency described in Sec. 6.7.4 as well as the spread in the neutron energy response, see Sec. 6.7.3.

Of course, since the hydrogen nucleus consists of a single proton, inelastic scattering on hydrogen is not possible.

\subsubsection{A typical fast neutron in SciBath}

The SciBath scintillator is primarily hydrogen and carbon. Two important features of the hydrogen and carbon cross sections in Fig. 2.5 are that the scattering cross sections are are orders of magnitude larger than the radiative capture cross sections and that the cross section for radiative capture on hydrogen is generally higher than for carbon, especially at low energies. Therefore a fast neutron that enters SciBath will scatter on hydrogen and carbon until it thermalizes. Once thermal, the neutron will continue elastically scattering without loosing energy until it radiatively captures, most likely on hydrogen.

Since the kinematics heavily favor energy transfer for elastic scattering with hydrogen over carbon, most of the neutron energy will be transferred to recoiling protons. Therefore a largely "diagonal" energy response response can be expected based on the known light output 


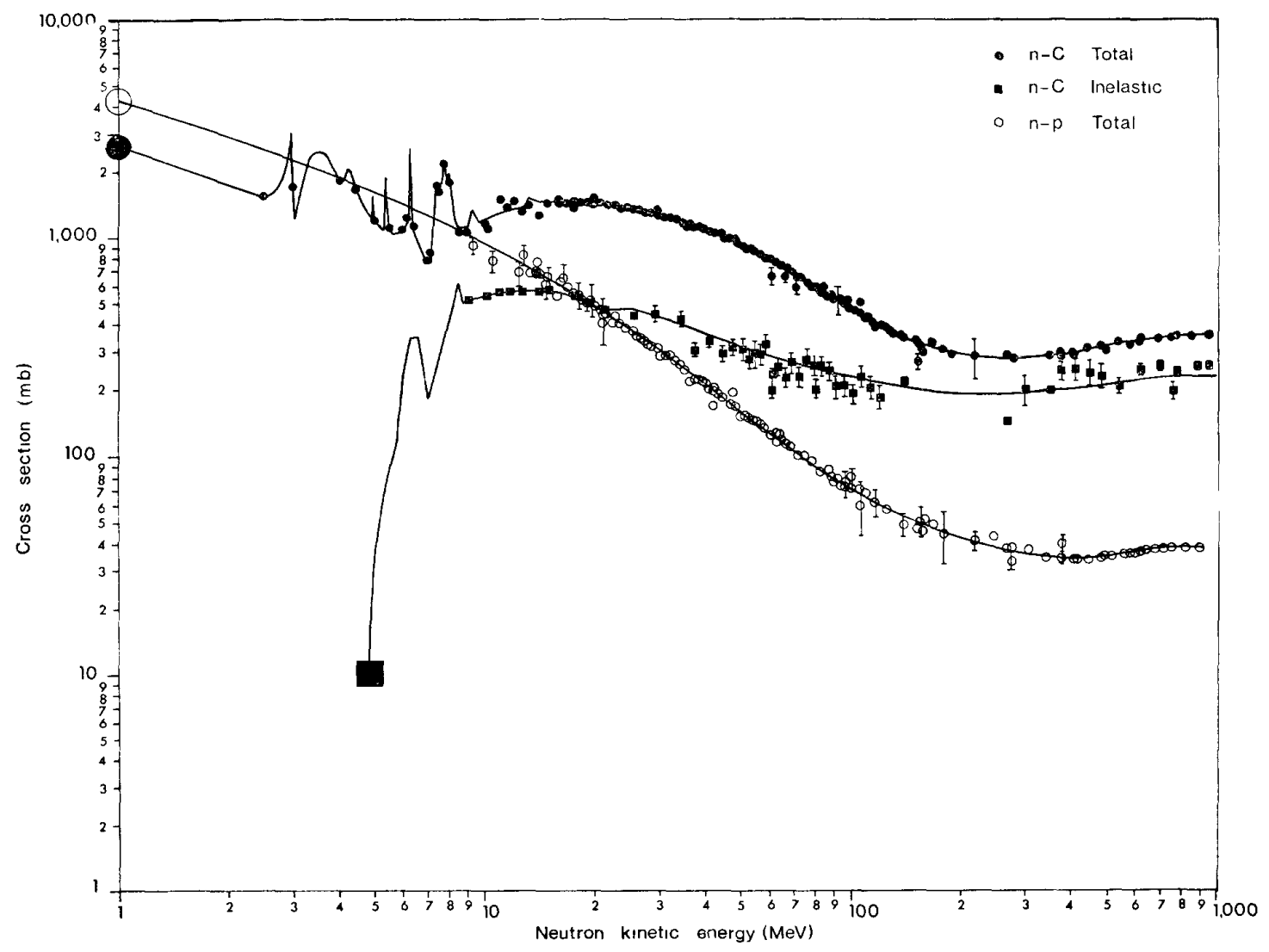

Figure 2.8: n-C total, n-C inelastic and n-p total cross sections data. The full lines show the cross sections used in the Monte Carlo program of Del Guerra [9]. Figure by Del Guerra.

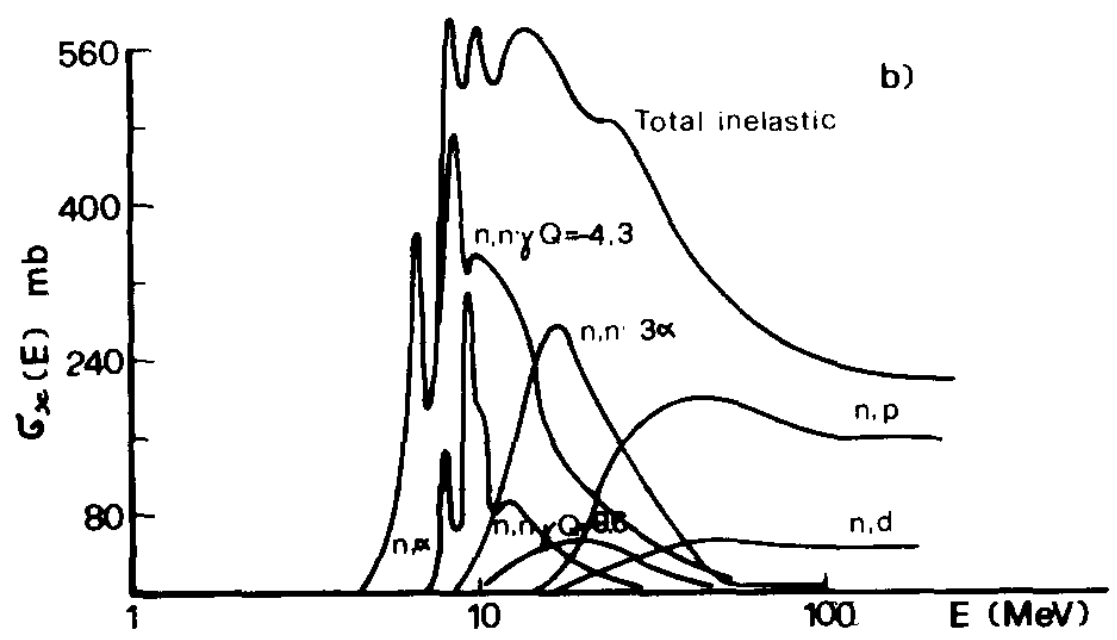

Figure 2.9: Cross sections for major neutron on carbon inelastic processes. Figure from Anghinolfi et al. [10]. 
of output of protons in liquid scintillator, see Sec. 6.3.2. Inelastic scattering on carbon does play a role in energy loss of neutrons above $10 \mathrm{MeV}$ and will degrade the neutron energy response somewhat. This degradation contributes to the smearing of the "diagonal" energy response in Fig. 6.15b.

Since the cross sections for elastic scattering are so much larger than radiative capture, a large number of scattering interactions occur before a neutron is captured. The neutron will deposit most on its energy in the first few interactions, which occur nearly instantaneously. The following scattering interactions take much more time simply because the neutron scatters so many times before capturing. This allows the common fast neutron detection criteria of one or more neutron-hydrogen scattering events followed by a $2.2 \mathrm{MeV}$ photon from neutron capture on hydrogen. The probable time between an initial neutron scatter and subsequent capture is described by a decaying exponential of characteristic time around $180 \mu$ s in the case of an infinite volume of liquid scintillator. Due to the finite size of SciBath and the fact that neutrons with longer delayed capture events are more likely to escape the detector, neutrons in SciBath have a characteristic time around 160 ps. This coincidence signal of a neutron scattering event followed by an appropriately delayed neutron capture event is the primary detection criteria for this analysis and is summarized in Fig. 2.10.

\subsection{Photons in SciBath}

Detection of the $2.2 \mathrm{MeV}$ photon resulting from radiative neutron capture on hydrogen is required for neutron detection in this analysis. The dominant photon interactions in matter are Compton scattering (coherent and incoherent), the photoelectric effect, and electronpositron pair production (in the field of a nucleus or an atomic electron). Figure 2.11 shows the cross sections for these interactions. Incoherent Compton scattering is clearly the dominant process at 2.2 MeV. The most basic incoherent Compton scattering occurs when 


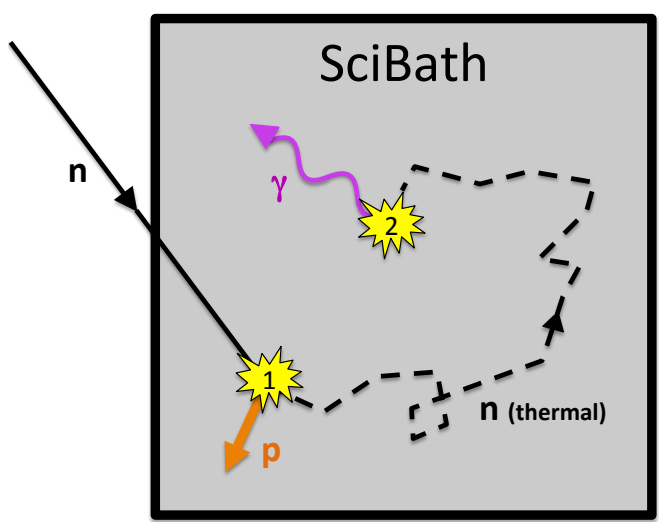

Figure 2.10: Neutron that scatters off of a hydrogen nucleus, thermalizes, and captures in SciBath. SciBath detects the recoiling proton followed by a $2.2 \mathrm{MeV}$ photon at a characteristic time near 160 ps.

a photon scatters off of a free electron. If the photon is relatively high energy compared to the binding energy of an atomic electron, however, the electron can be considered effectively free for Compton scattering. Since scattered electrons are charged, they produce detectable scintillation signals in SciBath. 


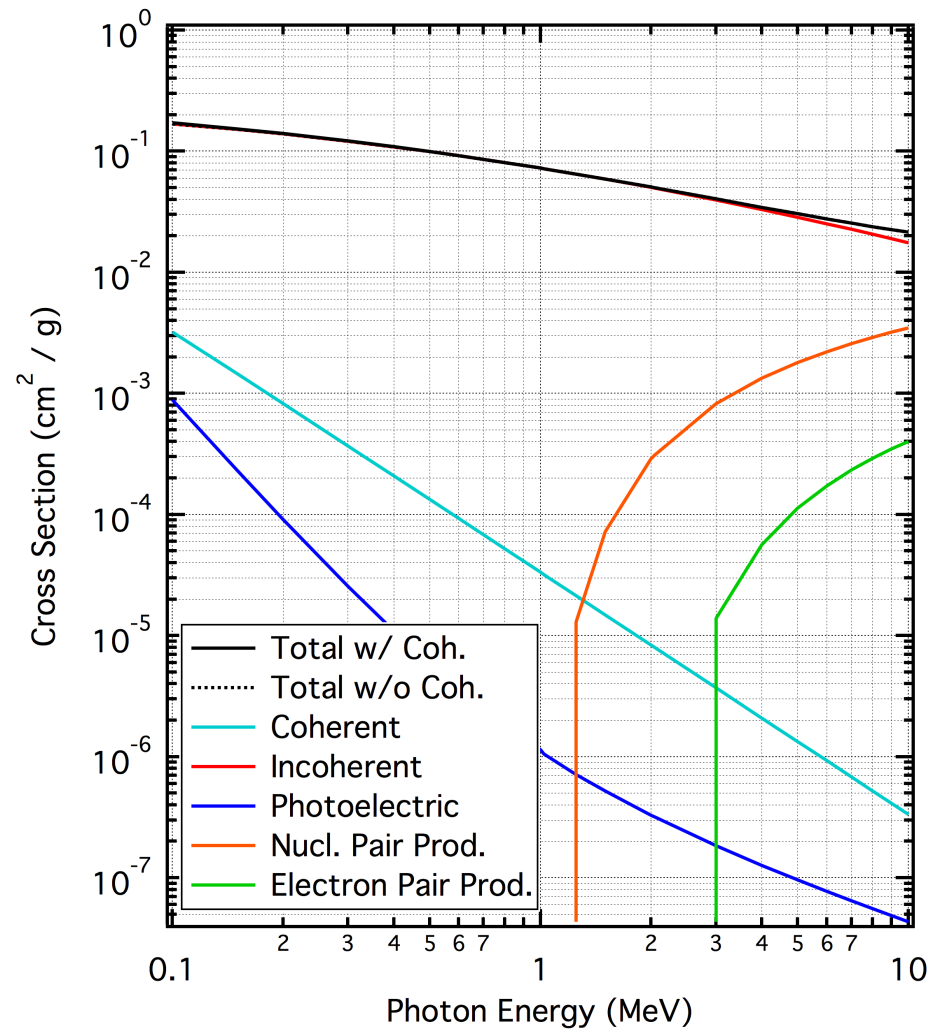

Figure 2.11: Cross sections for dominant photon interactions in liquid scintillator $\left(\mathrm{CH}_{2}\right)$. Incoherent Compton scattering is the dominant interaction for $2.2 \mathrm{MeV}$ photons from radiative neutron capture on hydrogen. Plot from the NIST XCOM database [11]. 


\section{Chapter 3}

\section{Previous Measurements and}

\section{Simulations}

In this chapter I describe previous measurements, simulations, and calculations of neutron and muon characteristics underground, with which our results can be compared. In the first section I summarize the many measurements of the muon flux at shallow depth and the relatively large uncertainty in its prediction at specific sites. The neutron production rate in scintillator has been measured by nine groups. I present their results and a proposed "universal" formula for these rates in Sec. 3.2. In the last section I describe estimates of the muon induced neutron flux at depth, which has only been studied at deep sites.

\subsection{Muon flux and angular distribution}

Muon fluxes underground have been studied extensively over the past 60 years at a variety of sites and depths. There is significant discrepancy between the results, however, and predictions of fluxes at unmeasured sites come with relatively large uncertainties. Figure 3.1 shows the world data as of 2008. Note that the depth in the figure is measured from the top of the atmosphere, which is roughly 10 meters water equivalent (m.w.e.) above the surface. 
The fit curve was derived by Barbouti and Rastin [28],

$$
I_{v}(h)=\frac{K}{\left(h^{\alpha}+a\right)(h+H)} \exp (-\beta h) \mathrm{cm}^{-2} \mathrm{~s}^{-1} \mathrm{sr}^{-1} .
$$

Barbouti and Rastin fit this form to data and found the parameters $K=270.7$ m.w.e., $\alpha=1.68, a=75, H=200$ m.w.e., and $\beta=5.5 \times 10^{-4}$ m.w.e. ${ }^{-1}$. Most of the data in Fig. 3.1 below 1000 m.w.e. was reported in 1971 or earlier.

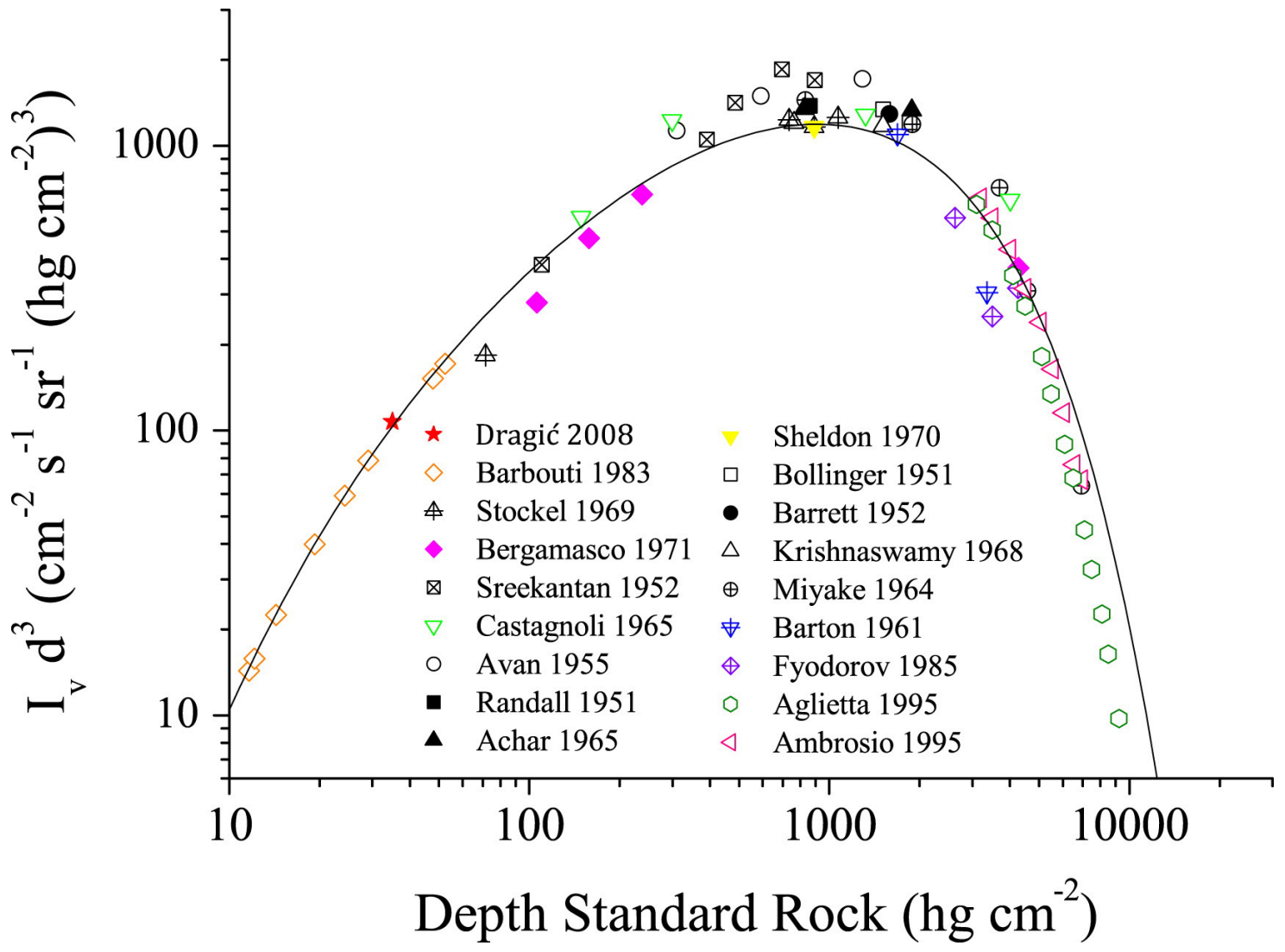

Figure 3.1: Absolute vertical muon intensity vs. depth measured from the top of the atmosphere. Diagram altered from Dragić et al. 12. The intensity is scaled by $\mathrm{d}^{3}$ to more clearly show the results. The depth unit hg $/ \mathrm{cm}^{2}$ represents the product of material density and muon path length; and when expressed as $\mathrm{hg} / \mathrm{cm}^{2}$ is approximately the same as meters water equivalent (m.w.e.).

Predictions of the muon flux at the NuMI depth of 265 m.w.e. from the surface $(275$ m.w.e. from the top of the atmosphere) are varied by 30\%. Equation 3.1 indicates a vertical muon flux of $0.389 \mathrm{~m}^{-2} \mathrm{~s}^{-1} \mathrm{sr}^{-1}$ at the NuMI depth. Multiplying this by the integral of 
the Miyake [13] angular distribution, Eqn. 3.2, over an upper hemisphere gives a predicted total muon flux of $0.86 \mathrm{~m}^{-2} \mathrm{~s}^{-1}$. Separately, Bogdanova et al. performed a calculation of muon fluxes at shallow depth [29]. Specifically, Fig. 2 in [29] predicts a vertical muon flux at $100 \mathrm{~m}$ of $0.33 \mathrm{~m}^{-2} \mathrm{~s}^{-1} \mathrm{sr}^{-1}$ and a total muon flux of $0.71 \mathrm{~m}^{-2} \mathrm{~s}^{-1}$. By comparison, Dr. Susan Kasahara simulated the cosmic muon flux specifically at NuMI for the MINOS Collaboration [30]. She found the muon flux through a unit horizontal area to be $0.77 \mathrm{~m}^{-2} \mathrm{~s}^{-1}$. The total muon flux, however, is the flux as seen by a spherical detector, not a horizontal plane. The difference between the angular distribution of the flux measured by these two geometries is a factor of $\cos \theta$ [31]. Multiplying the Kasahara result by the ratio of the integrals of the Miyake distribution with and without the extra $\cos \theta$ factor converts her result to a total muon flux of $1.03 \mathrm{~m}^{-2} \mathrm{~s}^{-1}$.

We consider the prediction fit curve by Barbouti and Rastin [28], Eqn. 3.1, to provide the most trustworthy prediction because of its comparison with a wide range of measurements. We assign this prediction a 30\% error based on the spread in the surveyed measurements and simulations at 265 m.w.e., resulting in a final muon flux prediction of $0.86 \pm 0.26 \mathrm{~m}^{-2} \mathrm{~s}^{-1}$. The muon flux result of this thesis is a direct, modern comparison with these previous results.

At the surface of the earth the accepted angular distribution for cosmic muons is $I(\theta) \approx$ $I_{\perp} \cos ^{2} \theta$, where $\theta$ is the zenith angle and the flux is uniform in the azimuthal angle. As depth underground increases the angular distribution becomes more vertical because higher $\theta$ muons travel through more rock and are thus more attenuated. For depths up to $100 \mathrm{~m}$ of standard rock, however, the distribution is well approximated by the surface distribution [29]. This is caused by the increase in the average energy of mouns at greater zenith angles [1, 32]. Miyake proposed a more precise form for the angular distribution at depth [13],

$$
I(h, \theta)=I(h, 0) \cos ^{1.53} \theta \exp \left(-8.0 \times 10^{-4} h(\sec \theta-1)\right),
$$

where $h$ is in m.w.e. Figure 3.2 shows a comparison between the Miyake distribution and 
the surface distribution.

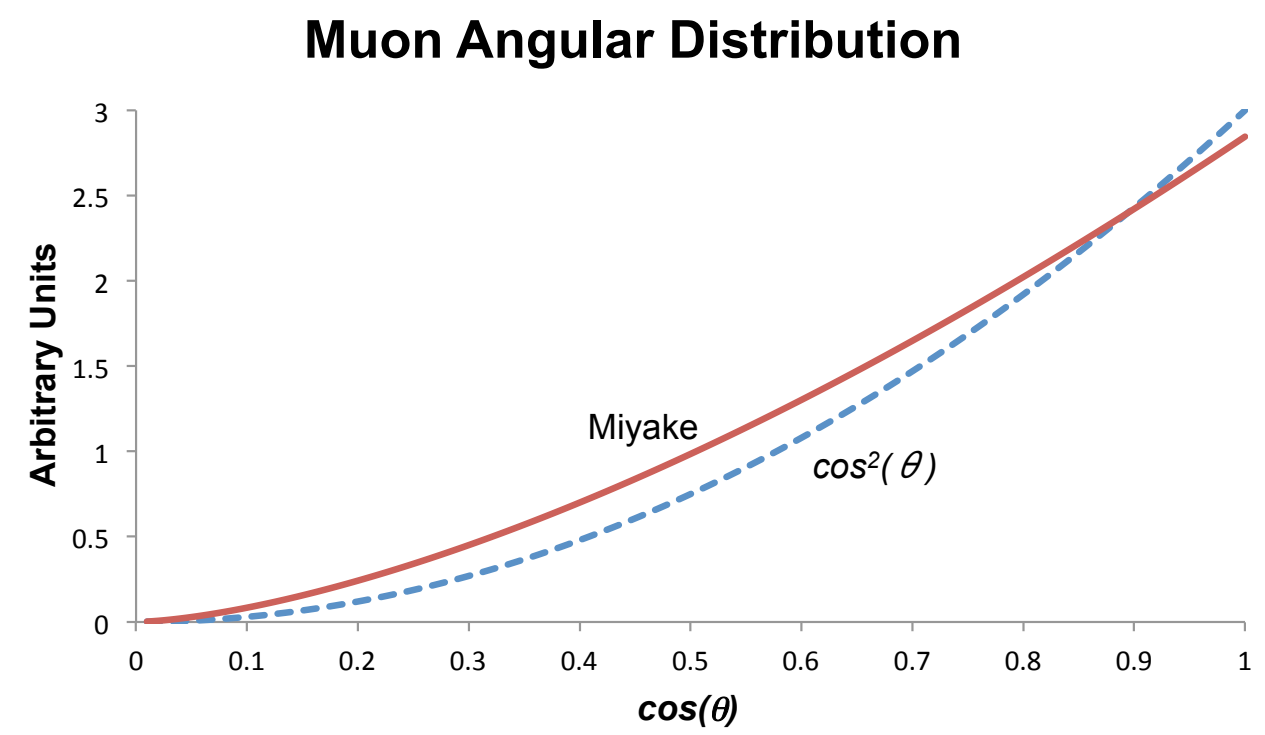

Figure 3.2: Surface muon angular distribution in comparison with the distribution proposed by Miyake [13] at 265 m.w.e.

\subsection{Neutron production rate through muon spallation}

The rate of neutron production through muon spallation in scintillator is the most studied characteristic of underground neutrons. The world data is presented in Table 3.1 and Fig. 3.3 , where some of the measurements have been corrected by Agafonova and Malgin [2]. Neutron yield increases with depth because average muon energy increases with depth. Agafonova and Malgin proposed a universal formula for the neutron yield from muons:

$$
Y_{n}\left(A, \bar{E}_{\mu}\right)=c A^{\beta} \bar{E}_{\mu}^{\alpha},
$$

where $A$ is the atomic weight of the target material and $\bar{E}_{\mu}$ is the average muon energy at the site. Agafonova and Malgin found the relevant parameters to be $c=4.4 \times 10^{-7} \mathrm{~cm}^{2} / \mathrm{g}$, $\beta=0.95$, and $\alpha=0.78$. 


\section{Neutron Yield in Scintillator Vs. Mean Muon Energy}

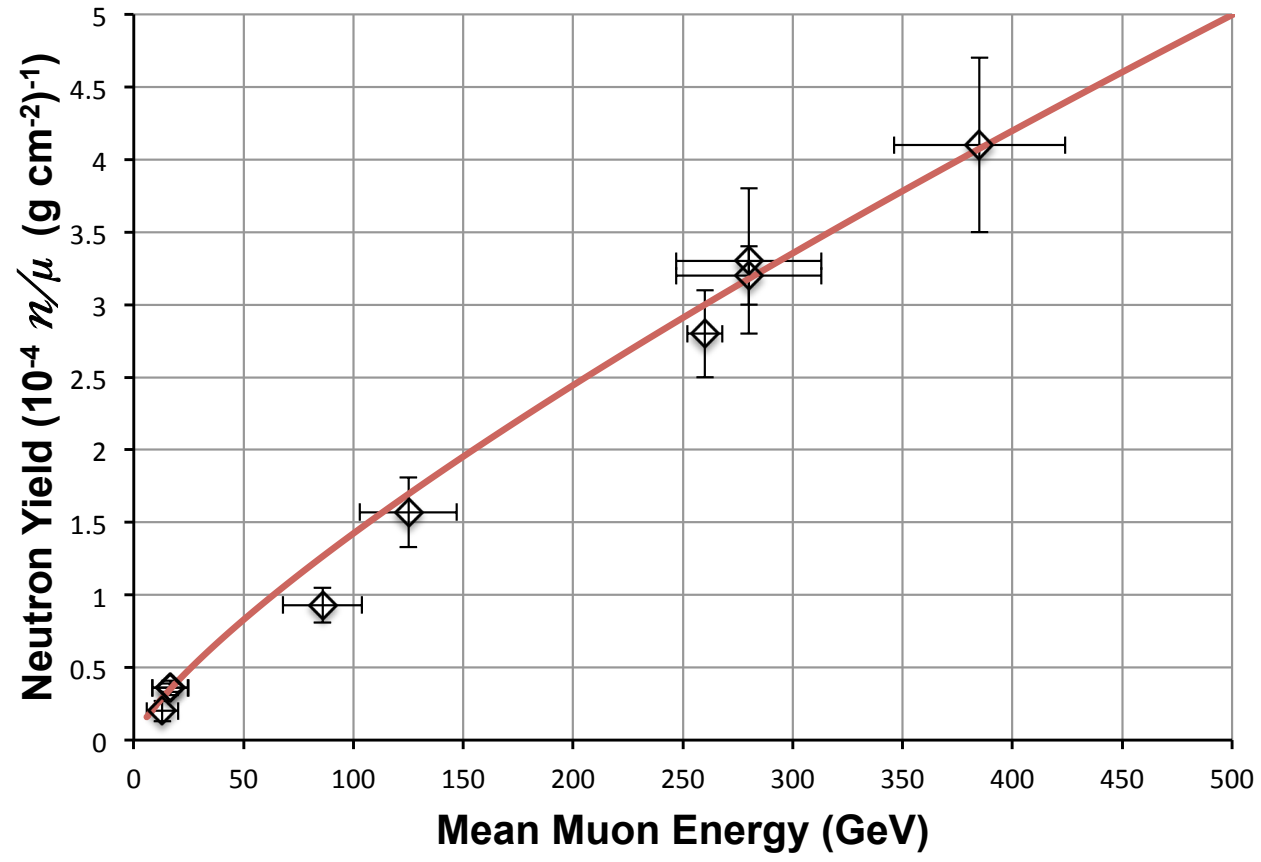

Figure 3.3: World data for neutron production in scintillator. The curve was proposed by Agafonova and Malgin [2], Eqn. 3.3. See Table 3.1 for references and identification of which results have been corrected by Agafonova and Malgin. 


\begin{tabular}{ccccc}
$\overline{\boldsymbol{E}}_{\boldsymbol{\mu}}(\mathbf{G e V})$ & $\mathbf{H}(\mathbf{m . w . e .})$ & $\boldsymbol{Y}_{\boldsymbol{L S}}\left(\times \mathbf{1 0}^{\mathbf{- 4}} \mathbf{n} / \boldsymbol{\mu}\left(\mathbf{g} / \mathbf{c m}^{\mathbf{2}}\right)^{\mathbf{- 1}}\right)$ & year & Ref. \\
\hline \hline $13.0 \pm 7.2$ & 20 & $0.20 \pm 0.07$ & 1995 & {$[33]$} \\
$16.5 \pm 8.1$ & 32 & $0.36 \pm 0.03$ & 2000 & {$[34]$} \\
$16.7 \pm 8.2$ & 25 & $0.36 \pm 0.05^{\dagger}$ & 1973 & {$[35]$} \\
$86 \pm 18$ & 316 & $0.93 \pm 0.12^{\dagger}$ & 1973 & {$[35]$} \\
$125 \pm 22$ & 570 & $1.57 \pm 0.24^{\dagger}$ & 1986 & {$[36]$} \\
$260 \pm 8$ & 2700 & $2.8 \pm 0.3$ & 2010 & {$[37$} \\
$280 \pm 33$ & 3100 & $3.3 \pm 0.5^{\dagger}$ & 2005 & {$[38$} \\
$280 \pm 33$ & 3100 & $3.2 \pm 0.2$ & 2011 & {$[39$} \\
$385 \pm 39$ & 5200 & $4.1 \pm 0.6^{\dagger}$ & 1989 & {$[40]$} \\
\hline
\end{tabular}

Table 3.1: World measurements of neutron yield in liquid scintillator. The ${ }^{\dagger}$ indicates measurements that were corrected by Agafonova and Malgin [2].

\subsection{Neutron flux and energy distribution}

The underground neutron flux from muon interactions in rock is poorly understood in general [14]. Background neutron flux measurements have been made underground, but only at sites that are deep compared to NuMI and usually at energies lower than $10 \mathrm{MeV}$. Mei and Hime provide a thorough examination of muon-induced backgrounds at sites greater than 1500 m.w.e. in [14]. In particular, their FLUKA simulations suggest the following expression for the neutron flux as a function of depth:

$$
\phi_{n}=P_{0}\left(\frac{P_{1}}{h}\right) \exp \left(-h / P_{1}\right),
$$

where $h$ is the vertical depth in km.w.e. and the Mei and Hime FLUKA simulations suggest fit parameters $P_{0}=(4.0 \pm 1.1) \times 10^{-7} \mathrm{~cm}^{-2} \mathrm{~s}^{-1}$ and $P_{1}=0.86 \pm 0.05 \mathrm{~km}$. w.e. This expression includes neutrons of all energies. By comparison, the flux measurement presented in this thesis only includes fast neutrons between 16 and $156 \mathrm{MeV}$.

The muon-induced neutron energy distribution is also poorly understood as the theoretical calculations and the few measurements give a wide range of results [41, 14, 42, 43, 44]. Further complicating the matter, the vast majority of measurements and calculations are for relatively large muon energies, and thus for deep underground sites. Mei and Hime pro- 
vide the most recent simulation of muon-induced neutron energy using FLUKA [14]. They suggest the following parameterization:

$$
\begin{gathered}
\frac{d N}{d E_{n}}=A_{\mu}\left(\frac{\exp \left(-a_{0} E_{n}\right)}{E_{n}}+B_{\mu}\left(\bar{E}_{\mu}\right) \exp \left(-a_{1} E_{n}\right)\right)+a_{2} E_{n}^{-a_{3}}, \\
B_{\mu}\left(\bar{E}_{\mu}\right)=0.324-0.641 \exp \left(-0.014 \bar{E}_{\mu}\right)
\end{gathered}
$$

where $A_{\mu}$ is a normalization constant; $\bar{E}_{\mu}$ is the mean muon energy; and $a_{0}, a_{1}, a_{2}$, and $a_{3}$ are fit parameters that depend on the specific site, see their Table VII. In particular for the WIPP site, the most shallow in the study at $1.585 \pm 0.011 \mathrm{~km}$.w.e., the parameters are $a_{0}=6.86, a_{1}=2.1, a_{2}=2.971 \times 10^{-13}$, and $a_{3}=2.456$. Mei and Hime also provide an expression for the mean muon energy at depth:

$$
\bar{E}_{\mu}=\frac{\epsilon_{\mu}(1-\exp (-b h))}{\left(\gamma_{\mu}-2\right)}
$$

where $h$ is the depth underground in $\mathrm{km}$.w.e. and the other parameters have been have been suggested by two different authors, see Table 3.2. Mei and Hime used Eqns. 3.5, 3.6, and 3.7 to fit their FLUKA simulated neutron energy spectra for various sites, see Fig. 3.4.

\begin{tabular}{ccccc} 
Ref. & $\boldsymbol{\epsilon}_{\boldsymbol{\mu}}(\mathbf{G e V})$ & $\boldsymbol{b}$ (km.w.e. $\left.{ }^{-\mathbf{1}}\right)$ & $\boldsymbol{\gamma}_{\boldsymbol{\mu}}$ & $\overline{\boldsymbol{E}}_{\boldsymbol{\mu}}(\mathbf{G e V})$ \\
\hline \hline [45] & 618 & 0.383 & 3.7 & 165 \\
[1, 16] & 693 & 0.4 & 3.77 & 184 \\
\hline
\end{tabular}

Table 3.2: Mean muon energy at WIPP and parameters relevant to its calculation with Eqn. 3.7 .

Most simulations and calculations of neutron fluxes underground, including those presented above, require three inputs: the muon flux and energy spectrum at depth, the muoninduced neutron production rate in scintillator for muons of the appropriate mean energy, and the material dependence of the muon-induced neutron production rate. Each of these inputs are based on measurements, but their uncertainties are relatively large. The fast neutron flux measurement and energy distribution presented in this thesis will add a result that 


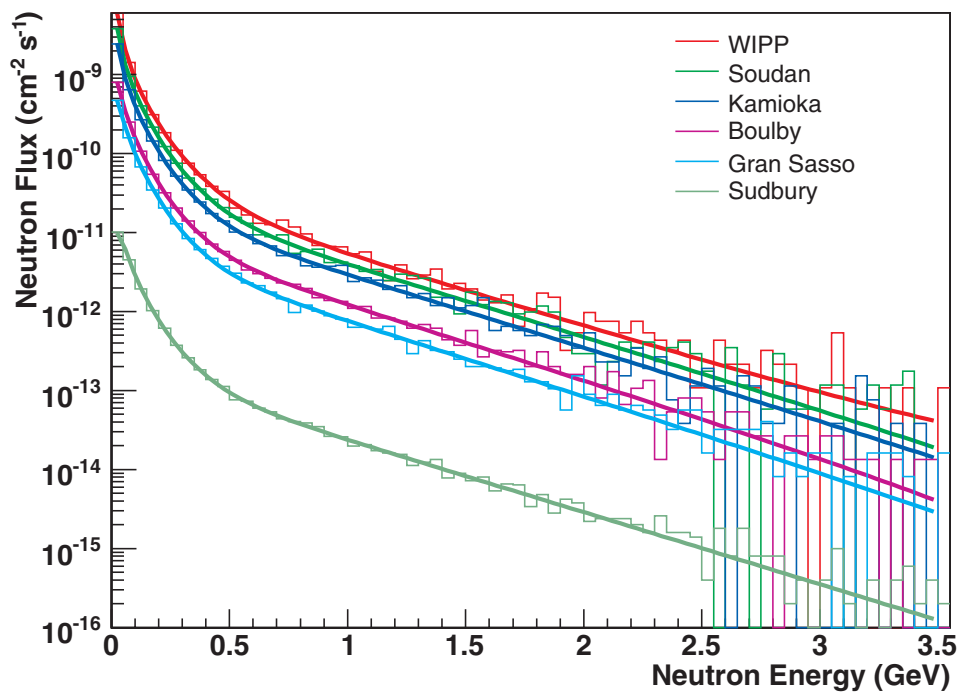

Figure 3.4: The differential energy spectrum for muon-induced neutrons at various underground sites. The plot was made by Mei and Hime [14].

is unique in depth and energy. This will constrain the models used to predict fast neutron fluxes underground and therefore improve neutron background estimates for current and future experiments. 


\section{Chapter 4}

\section{The SciBath Detector}

In this chapter I describe the SciBath detector. I first discuss the original inspiration as a prototype neutrino detector and give an overview of the SciBath operating principle as a liquid scintillator detector with a grid of readout optical fibers. I then go into more detail about the specific scintillator and optical fibers that were used to carry signals to the data acquisition system, which digitizes these signals and handles data flow and organization in preparation for analysis. In Sec. 4.7 I describe the SciBath LED calibration system before describing the SciBath plumbing system, which was responsible for both liquid scintillator and nitrogen gas handling, in Sec. 4.8. I end the chapter with a description the SciBath run monitoring systems. A schematic and photograph showing major SciBath components can be seen in Fig. 4.3 .

\subsection{FINeSSE}

SciBath is a prototype of the proposed FIne-grained Neutrino Scattering Scintillator Experiment (FINeSSE) [46]. FINeSSE was designed to take measurements of the cross sections of the Neutral Current Elastic (NCel) and Charge Current Quasi-Elastic (CCQE) neutrino interactions shown in Fig. 4.1. The ratio of these cross sections provides a direct measurement of the strange quark contribution to the proton spin, $\Delta S$, but requires a fine-grained 
detector for the precise reconstruction of outgoing 100 to $500 \mathrm{MeV}$ proton tracks, which travel $\mathcal{O}(10) \mathrm{cm}$ in liquid scintillator. Unfortunately, neutrinos only interact through the weak force, so interactions are rare and a large volume of scintillator is required. A quick calculation noting that typical neutrino production rates are $10^{6} \nu \mathrm{cm}^{-2} \mathrm{~s}^{-1}$ and typical cross sections are $\sigma \sim 10^{-39} \mathrm{~cm}^{2}$ gives a required detector of $\mathcal{O}(10)$ ton for a few interactions per minute. The FINeSSE design was intended to tackle this challenge of an economically viable $\mathcal{O}(10)$ ton detector with fine-grained tracking. SciBath will be used to demonstrate the FINeSSE concept for a future proposal.

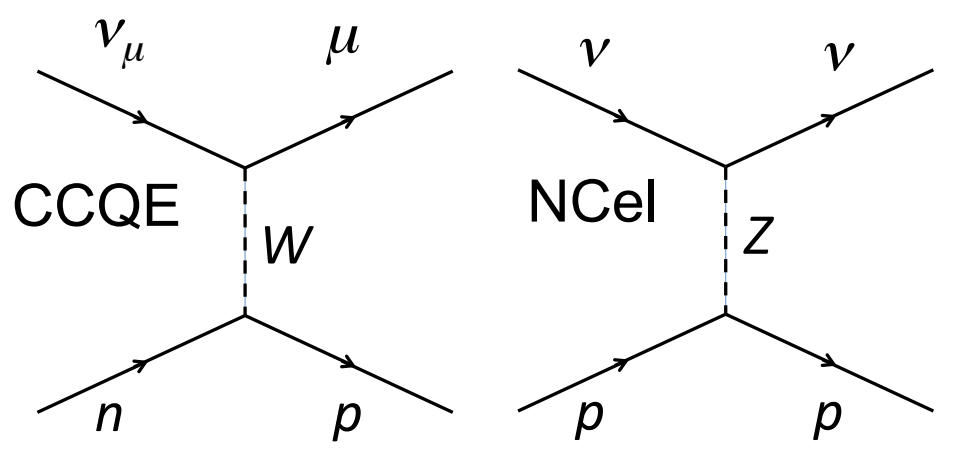

Figure 4.1: Feynman diagrams for the Charge Current Quasi-Elastic (CCQE) and Neutral Current elastic (NCel) neutrino interactions whose cross sections FINeSSE was designed to measure.

\subsection{Overview and principle of operation}

The SciBath concept consists of a large, optically-open bath of liquid scintillator, which also provides a target mass near $70 \mathrm{~kg}$. High-energy neutral particles such as neutrinos, neutrons, and photons then interact with the target mass and produce recoiling charged particles, typically electrons, muons, or protons. These charged particles then induce scintillation in the same target mass due to the added scintillator. The scintillation light is captured by a periodic array of wavelength-shifting (WLS) fibers, which then re-emit light within their interiors and guide it to a readout array of photomultiplier tubes (PMTs). The choice of 
scintillator and WLS fibers is intended to induce Stokes shifts to avoid strong attenuation and to match the peak quantum efficiency spectrum of the PMT to maximize the signal. A schematic of this process is shown in Fig. 4.2 .

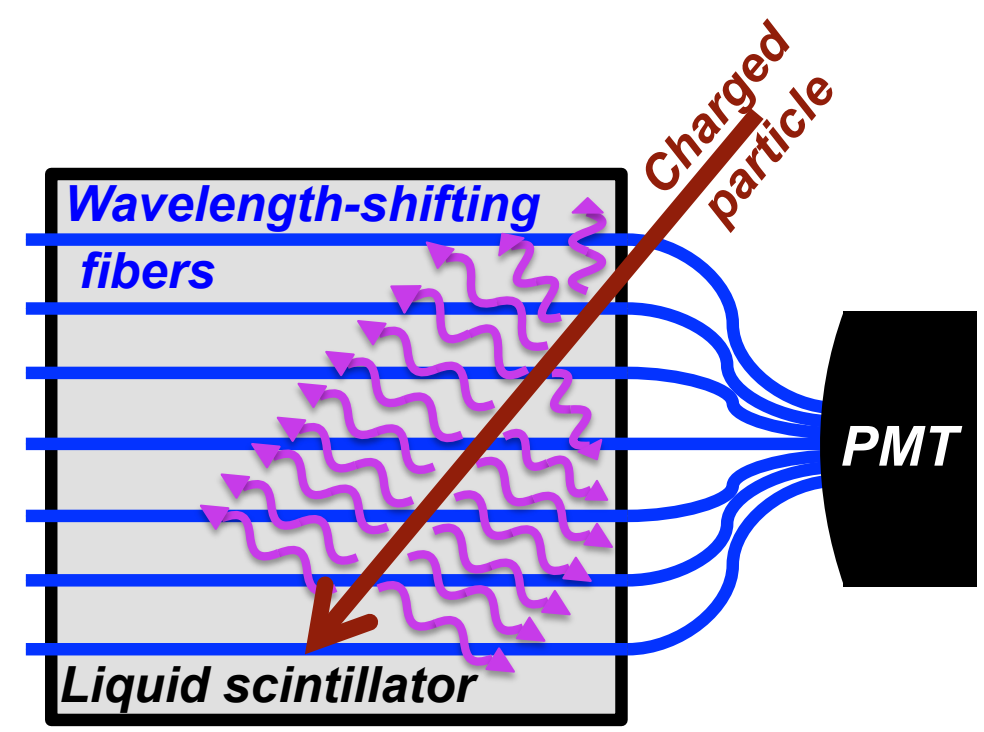

Figure 4.2: Schematic showing the SciBath event detection principle for charged particle detection. For a neutral particle to be detected it must interact in the scintillator and create charged particle tracks.

An overview of the SciBath detector itself is shown in Fig. 4.3. The active liquid scintillator is a $(0.45 \mathrm{~cm})^{3}$ cube containing $82 \mathrm{~L}$ of mineral oil based liquid scintillator. The volume is read out by 768 WLS fibers where each cardinal axis is read out by a square $16 \times 16$ array with one-inch spacing. Figure 4.4 shows schematics of the WLS fiber arrangement and their placement inside the detector cube. Each fiber penetrates opposite ends of the 0.5 inch thick aluminum walls of the detector cube. One end is glued with Emerson and Cuming STYCAST 1266 epoxy to a brass plug which is secured to one aluminum wall, while the other end is glued to a stainless steel piston that is mounted to the opposite wall and is free to slide within a pair of rubber O-rings. The O-rings seal the detector against leaks, and the piston motion allows for approximately 1 psi over-pressure to maintain fiber tension within the cube. A purged $\mathrm{N}_{2}$ environment provides this overpressure and reduces gaseous oxygen poisoning, which degrades light output in liquid scintillator. The entire liquid volume can 
be contained in an onboard $104 \mathrm{~L}$ reservoir, and the entire detector is easily transportable as shown in Fig. 4.5 .
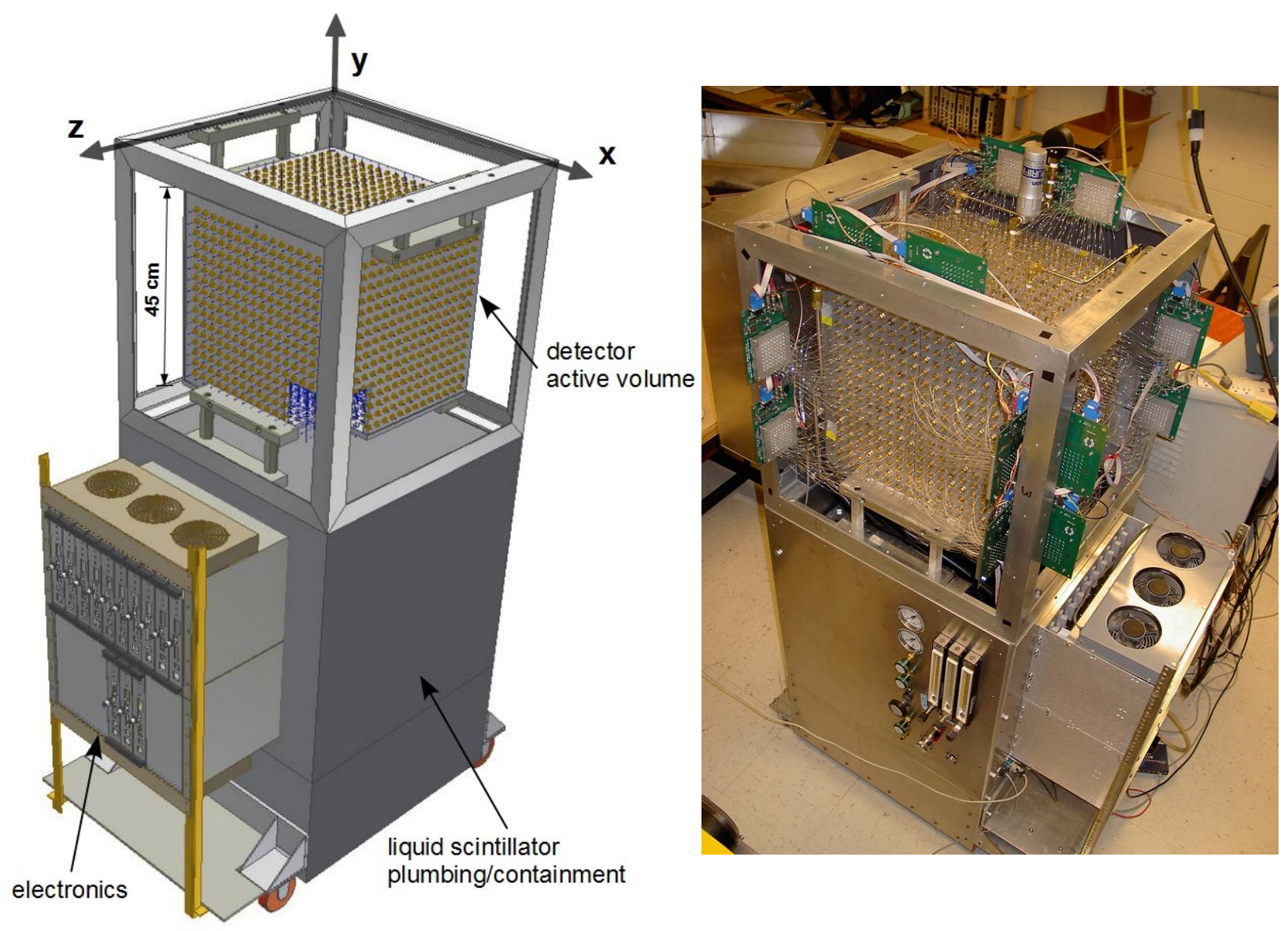

Figure 4.3: Schematic and photographic overviews of the the SciBath Detector. The SciBath detector cube and optical fibers are usually covered by protective panels, but those were removed for this photograph. The LED calibration system (green boards) can be seen as well as the optical fibers that guide their light to WLS fibers in the cube.

\subsection{Scintillator}

The liquid scintillator used in SciBath was mineral-oil based with $15 \%$ pseudocumene $(1,2,4$ Trimethylbenzene, $\left.\mathrm{C}_{9} \mathrm{H}_{12}\right)$ by volume and $1.5 \mathrm{~g} / \mathrm{L}$ PPO (2,5-Diphenyloxazole, $\left.\mathrm{C}_{15} \mathrm{H}_{11} \mathrm{NO}\right)$. This mixture was homemade and is very similar in composition to commercially available liquid scintillators EJ-321L [47] and BC-517 H [48]. Our liquid scintillator does not contain 


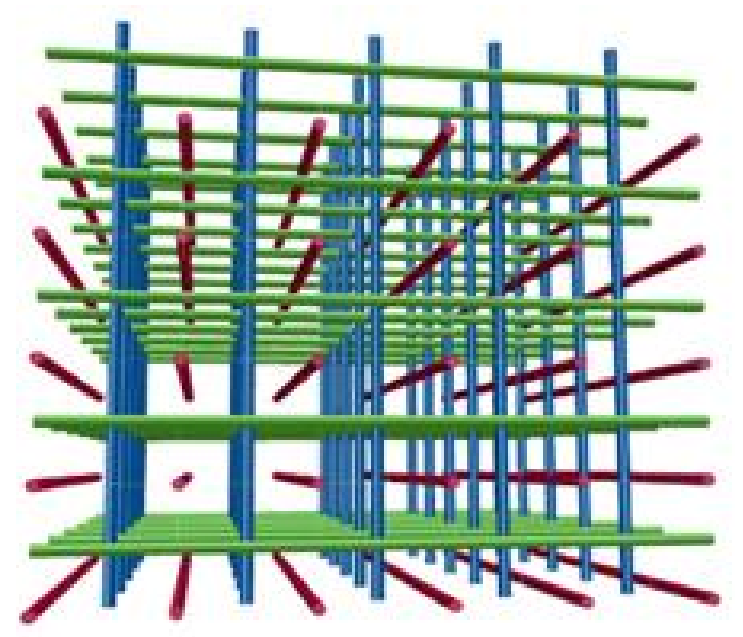

(a) Wavelength-shifting (WLS) fiber grid schematic.

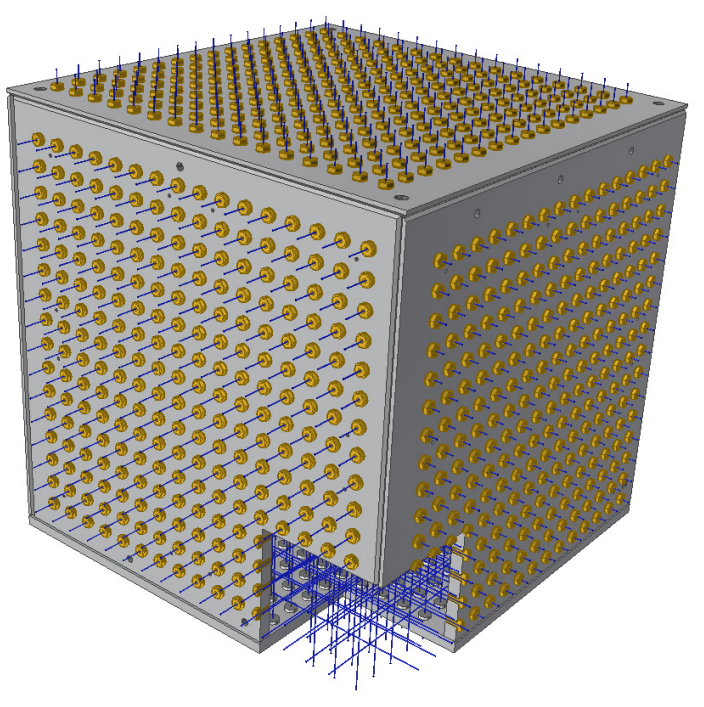

(b) Detector cube schematic.

Figure 4.4: Schematics of the WLS fiber grid and the SciBath detector cube. The lower front corners of the cube walls have been removed, exposing the WLS fibers inside.

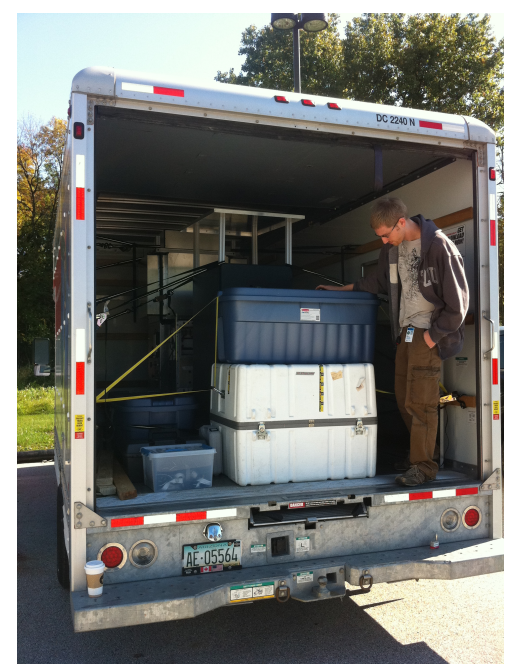

Figure 4.5: The SciBath detector, data acquisition system, and supporting equipment loaded in a moving truck for deployment at FNAL. 
tertiary wavelength shifters (e.g. bis-MSB, POPOP) like the commercial products. These are added to increase attenuation lengths to $\mathcal{O}(5) \mathrm{m}$ for large volume, long-optical-path-length detectors. The optical path-length for optimal performance of SciBath is only $\mathcal{O}(10) \mathrm{cm}$. For more details regarding the liquid scintillator selection and composition see Ref. [49].

Without wavelength shifters in the liquid, the peak scintillation emission wavelength is in the UV near $370 \mathrm{~nm}$, see Fig. 4.6. The attenuation length for scintillation light is $\gtrsim 1 \mathrm{~m}$ which is adequate for SciBath WLS fiber detection and tracking.

The scintillator is in large proportion mineral oil whose density is $0.86 \mathrm{~g} / \mathrm{cm}^{3}$ and index of refraction is $n=1.476$. The flashpoint of pseudocumene is $54.4^{\circ} \mathrm{C}$, which is low enough to be considered a flammable liquid. Dilution with the relatively inert mineral oil ameliorates flammability concerns. As a potentially flammable liquid, between two and four levels of leak protection are used, three of which could contain all $104 \mathrm{~L}$ of liquid from the reservoir.

The scintillator is continuously circulated between the cube and reservoir under a $\mathrm{N}_{2}$ environment to prevent oxygen poisoning from quenching the scintillator light output. For electron recoils and minimum ionizing muons, the scintillator light output was assumed to be linear over the SciBath energy sensitivity range throughout the NuMI run. The light yield is approximately 40-50\% anthracene, yielding approximately 7000 photons per $\mathrm{MeV}$ energy deposit.

\subsubsection{Notes on mixing}

Pseudocumene is toxic to humans and the environment [50]. Eyeshields, faceshields, and a respirator or fume hood are recommended during handling. Pseudocumene is also chemically aggressive and will dissolve plastic and rubber given enough time. This high reactivity was utilized during scintillator mixing as the pseudocumene was used to completely dissolve the PPO before adding the mix to mineral oil, which does not readily dissolve PPO. 


\subsection{Wavelength shifting fibers}

Custom St. Gobain BCF-99a [48] WLS fibers were used to convert the near UV scintillation light to blue to better match the peak quantum efficiency of the PMT. Incident UV scintillation light was absorbed in the core of the WLS fibers whose absorption spectrum is peaked at $\lambda=345 \mathrm{~nm}$. Figure 4.6 compares this absorption spectrum to the emission spectrum of a liquid scintillator similar to ours, peaked at $370 \mathrm{~nm}$. Fluorescent light is re-emitted isotropically inside a WLS fiber, and a small amount of fluorescent dye ( $200 \mathrm{ppm})$ is sufficient to Stokes shift the light to an emission spectrum that is peaked at $\lambda^{\prime}=435 \mathrm{~nm}$. The Stokes shift is required to avoid self-absorption along the fiber, and the level fluorescence permits attenuation lengths $\gtrsim 1 \mathrm{~m}$. The WLS fiber shifted emission spectrum should be compared to our photomultiplier tube efficiency spectrum, see Fig. 4.6.

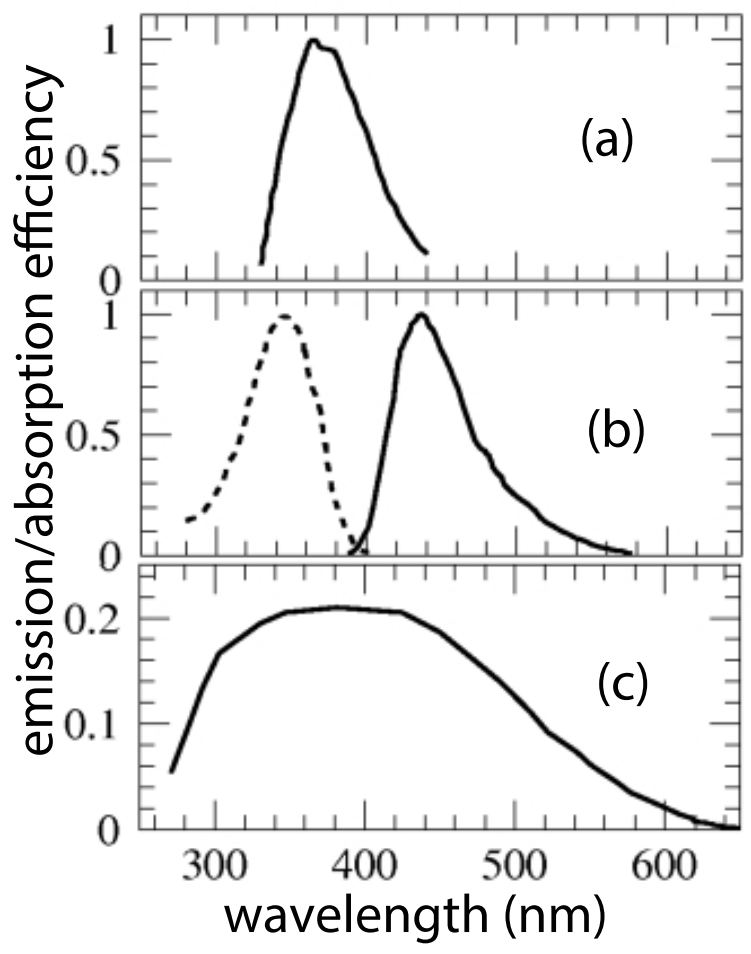

Figure 4.6: a) Emission spectrum for EJ scintillator (similar to ours), b) absorption (dotted) and emission (solid) spectra of our (UB) WLS fibers, c) quantum efficiency of our PMTs.

The WLS fibers have a single polystyrene core with a $1.38 \mathrm{~mm}$ inner diameter and 
an index of refraction $n=1.60$. Their inner cores are surrounded by a single layer of polymethylmethacrylate (PMMA) which is $45 \mu \mathrm{m}$ thick and has an index of refraction of $n=1.49$, and this is cladded with a second layer of fluorinated polymer with a thickness of $15 \mu \mathrm{m}$ and an index of refraction of $n=1.42$. The total capture fraction of fluorescence photons produced at the center of a WLS fiber is $5.6 \%$, and this rises if the emission point is closer to the step-index boundary of the cladding due to helical orbits. When convolved with the attenuation length and halved due to the fact that we only read out one end of the fibers, we expect a total capture fraction of approximately $3.75 \%$.

\subsection{Optical fibers}

The WLS fibers were coupled and glued to clear $\sim 1 \mathrm{~m}$ optical fibers (St. Gobain BCF-98) outside the $\mathrm{Al}$ detector cube. These fibers guided WLS fiber emissions to photomultiplier tubes (PMTs) with effectively no attenuation. Each end of these fibers was fly cut with a diamond-edged cutter to allow the best possible light transmission. The coupling to WLS fibers utilized precisely machined $1 \mathrm{~cm}$ brass hollow cylinders that held the ends of the two fibers together. To make this connection simpler, the optical fiber diameter was chosen to match that of the WLS fibers. Nye OC-431A-LVP optical coupling gel was placed between the mating fibers to further encourage optical transmission. The fiber coupling was mechanically reinforced with low melt-temperature hot glue wrapped in aluminum foil, which allowed us to squeeze the hot glue along the brass cylinder and $\sim 1 \mathrm{~cm}$ of each fiber. The 64 fibers from each quadrant of each detector axis were bundled into a $16 \times 16$ array and moulded into a StyCast square that was precisely aligned with the face of a 64-anode PMT.

\subsection{Data acquisition system}

The data acquisition system (DAQ) of a detector is responsible for converting physical phenomena into digital numeric values that can be analyzed by a computer. In the case of 
scintillator detectors, the physical phenomena is scintillation light. In this section I describe the photomultiplier tubes that digitize scintillation light signals as well as the readout electronics that perform zero suppression and handle data flow. I then go on to describe the trigger modes in which the DAQ can operate, an extra "break-in" board that was used for external signal input, and the method for clock synchronization of the readout electronics. Figure 4.7 shows SciBath and its DAQ system in data-taking mode.

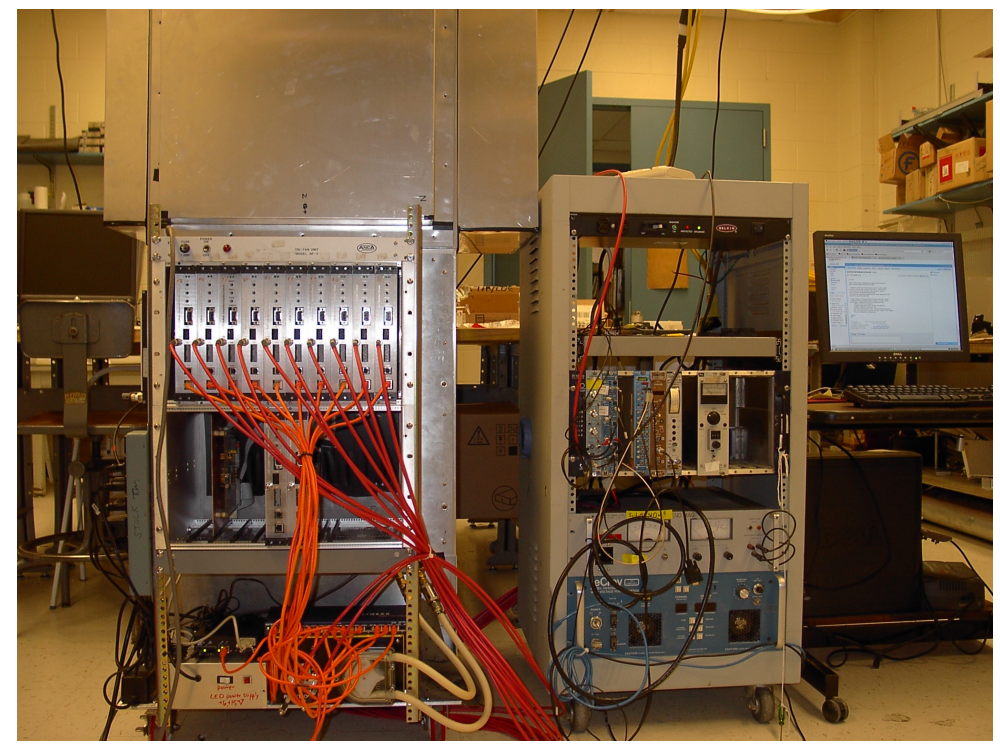

Figure 4.7: The SciBath detector with its data acquisition system complete for data-taking. Pictured from left to right is the SciBath detector, a half rack with power supplies and trigger logic, and the data acquisition computer.

\subsubsection{Photomultiplier tubes}

WLS fiber signals are converted to electronic signals by twelve Hamamatsu R7600-00-M6464anode photomultiplier tubes (PMTs) [51]. Each PMT contains 64 channels packaged into in an $8 \times 8$ array on a $25.7 \mathrm{~cm} \times 25.7 \mathrm{~cm}$ square, with each channel at $2 \times 2 \mathrm{~mm}$. These PMTs contain bialkali photocathodes sealed with borosilicate glass windows, and the wavelength sensitivity is $300 \mathrm{~nm}$ to $650 \mathrm{~nm}$ peaking at $420 \mathrm{~nm}$ with a maximum quantum efficiency in vacuum of $23 \%$, see Fig. 4.6. PMT "base" boards mount directly to the PMTs and provide 
the voltage divider circuitry. These boards were designed and assembled on-site at Indiana University, though the actual PCBs were manufactured off-site. Figure 4.8 shows a PMT next to its "base" board.

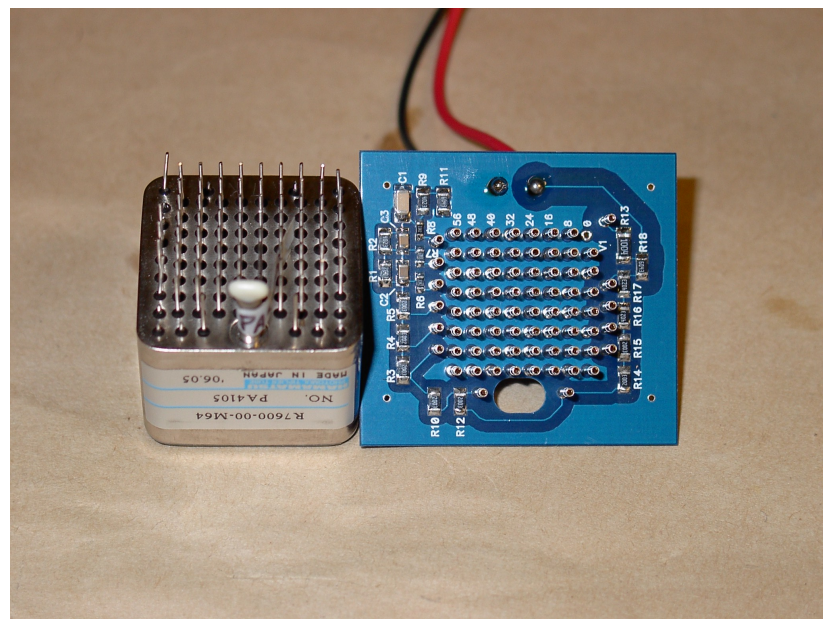

Figure 4.8: Hamamatsu 64 anode photomultiplier tube (face down, left) next to its voltage divider "base" board (right), designed at Indiana University.

\subsubsection{Integrated Readout Modules}

Each PMT is mounted to an "Integrated Readout Module" (IRM), which can be seen in Fig. 4.9 and Fig. 4.10 and serves as both the digital readout and physical mounting for the PMT. The IRMs are Indiana-University designed, custom-built digitizers. They are housed in an empty VME crate shell, but do not utilize standard VME power or connectivity. Instead, the IRMs are externally powered and connectivity is established through 1-Gigabit ethernet protocol. Figures 4.11 and 4.12 show the crates without and with mounted IRMs, respectively. Each IRM digitizes 64 channels with 12-bit, $20 \mathrm{MHz}$ flash analog-to-digital converters (ADCs). Additional parsing and signal logic is performed with onboard FPGAs and an ARM-9 microcontroller with an embedded linux kernel.

Fast PMT pulses are integrated and stretched in time with a "ringing-oscillator" shaping circuit on the front-end DAQ electronics. This allows a single ADC to measure both the amplitude and time of an incoming signal. The number of pre-trigger and total samples 


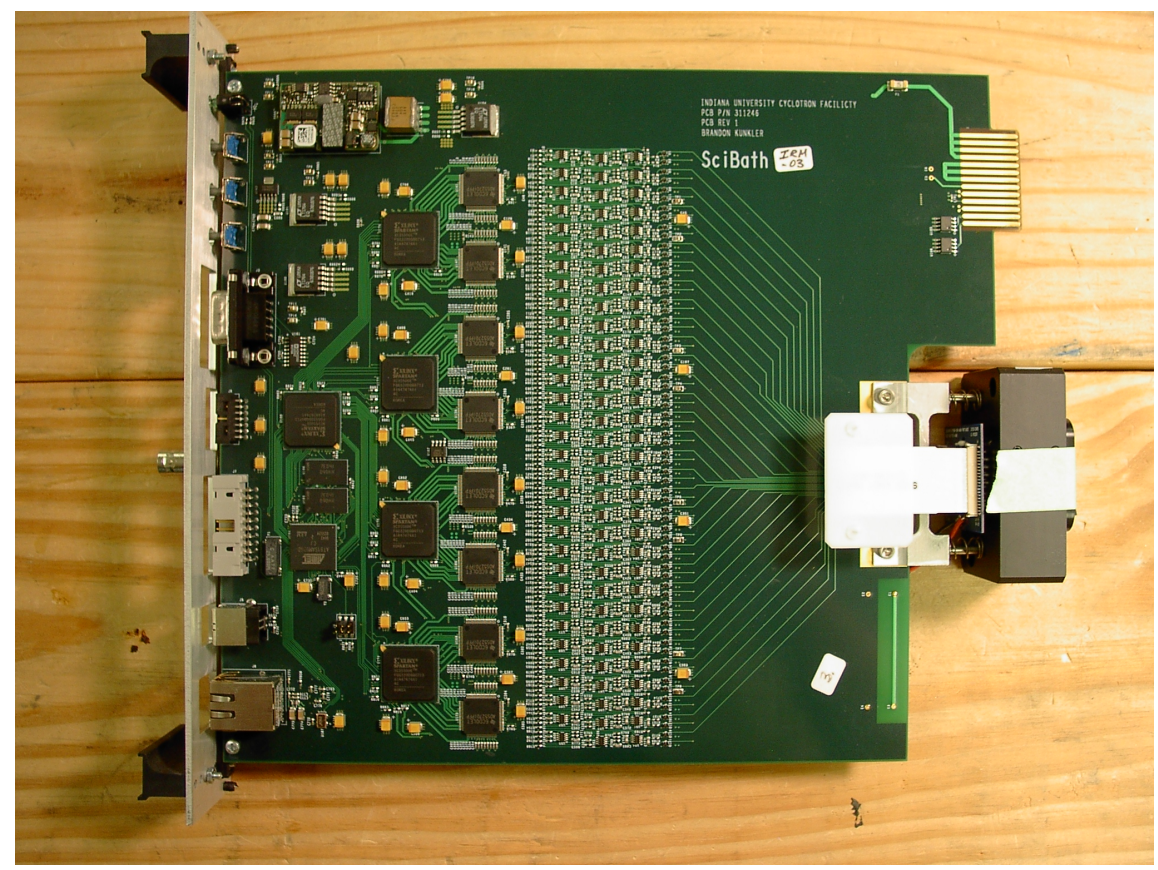

Figure 4.9: An integrated readout module (IRM). SciBath uses 12 IRMs to read out the detector and perform low level analysis and buffering. Each IRM reads out 64 wavelengthshifting fibers and has an integrated 64-anode photomultiplier tube (shown on the right side of the board).

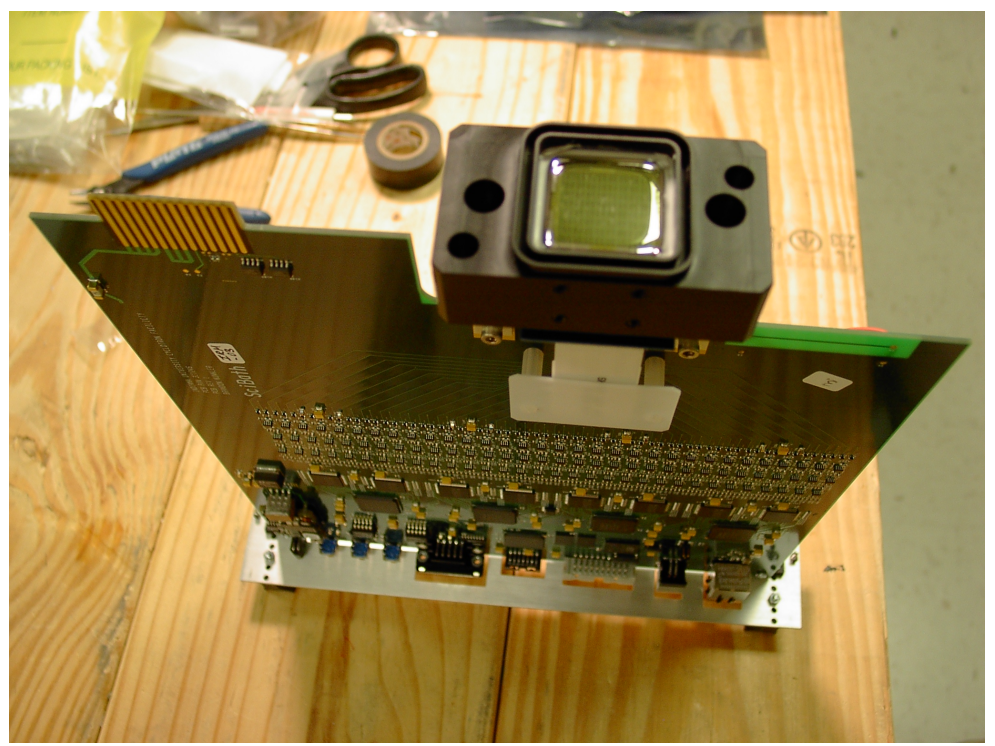

Figure 4.10: A PMT mounted to an IRM. The PMT housing ensures proper alignment with the corresponding readout fiber bundles. 


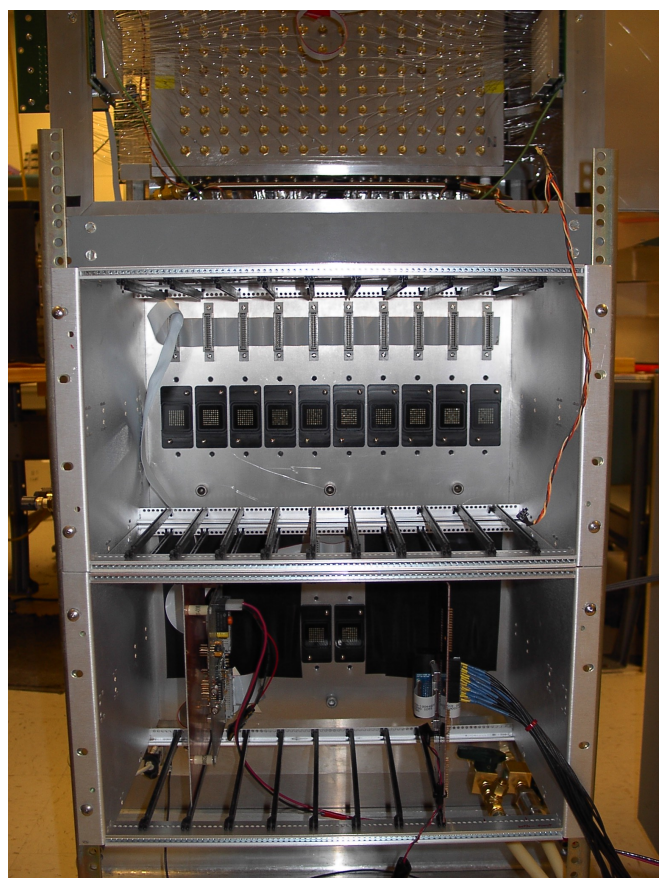

Figure 4.11: Two VME crate "shells" that hold the integrated readout modules on the SciBath detector and and align their PMTs with readout fiber bundle cookies (the black rectangles). Also pictured is a board (lower left) that regulates power and clock synchronization signals to the integrated readout modules through a ribbon cable.

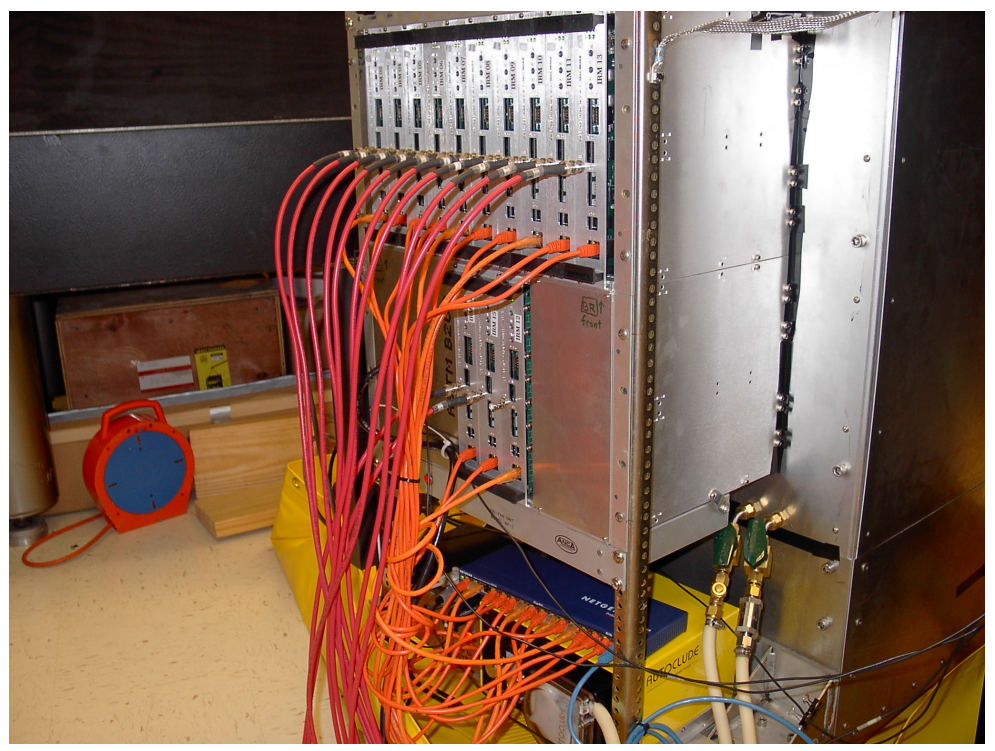

Figure 4.12: VME crate "shells" filled with the full set of readout electronics, complete for data-taking. Cables shown include high voltage to photomultiplier tubes (red) and ethernet cables for data transfer (orange). 
per waveform pulse are customizable at runtime. Throughout the NuMI run, 5 pre-trigger pulses and 20 total samples $(20 \cdot 50 \mathrm{~ns}=1 \mu \mathrm{s})$ were recorded. Figure 4.13 shows two sample waveforms.
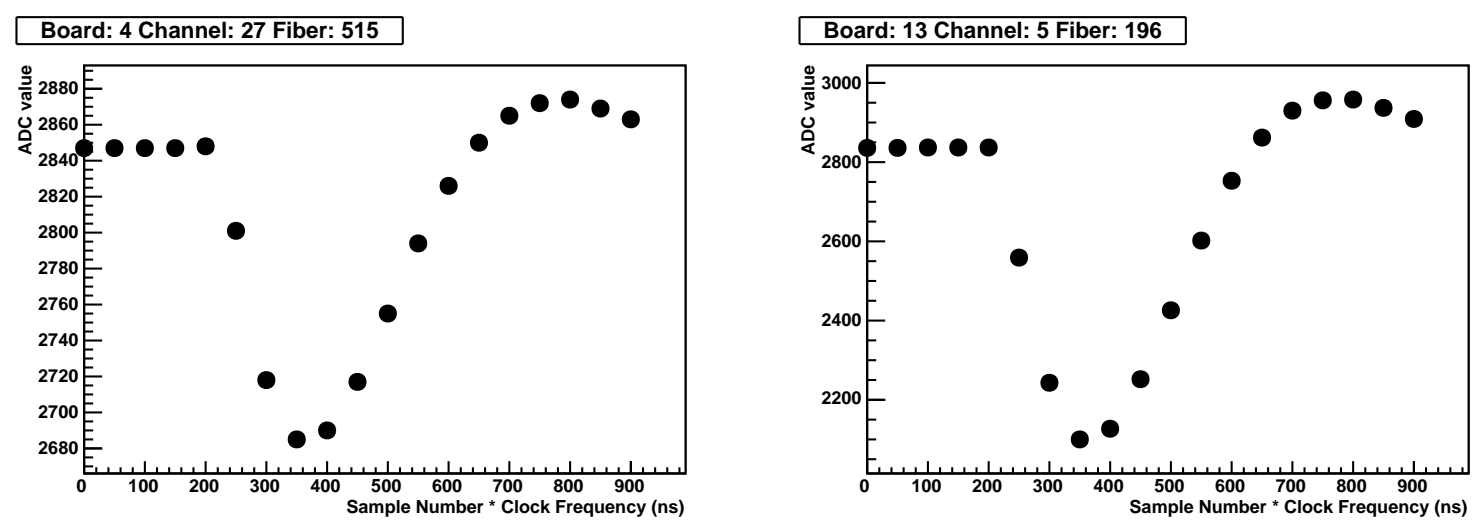

Figure 4.13: Two sample photomultiplier tube waveforms in SciBath. The standard photomultiplier tube signals have been modified by "ringing oscillator" shaping circuits.

\subsubsection{Trigger modes}

The DAQ can be operated in a variety of triggering modes. When internally triggered, the DAQ can be gated by an external NIM logic pulse. In this mode data recording is triggered for a particular channel when its ADC samples pass zero suppression logic, which requires the ADC voltage difference between two samples $100 \mathrm{~ns}$ apart to be greater than the trigger level. This trigger level can be individually set for each IRM, but due to gain matching the trigger level is generally held constant. Zero suppression can be turned off (no triggering) and the DAQ externally triggered, which is useful during LED calibration.

\subsubsection{Break-in board}

An additional IRM "break-in" board was constructed to accommodate up to 7 additional auxiliary signals into the DAQ data stream. The break-in board was used to record the various beam triggers during our NuMI and MI-12 deployment that were used to construct 
our trigger. Additionally, this board added beam trigger signals to our data, allowing timing cuts with reference to NuMI beam pulses. Though initially designed to handle NIM standard logic pulses, we found very little signal shape degradation and spectroscopic measurements have been made on liquid scintillator samples, though not in connection with this analysis.

\subsubsection{Integrated Readout Module clock synchronization}

Each IRM has its own internal clock, derived from a $25 \mathrm{MHz}$ crystal oscillator, used to drive its FPGAs, memory, etc. However, since data from a single event is handled by multiple boards, the clock that drives the $20 \mathrm{MHz}$ flash ADCs must be common between boards and a common synchronized counter must be implemented. This is accomplished via an additional home-built board that is housed in the VME crates. This "clock-board" distributes this common $20 \mathrm{MHz}$ clock on a simple ribbon cable bus along with $48 \mathrm{~V}$ power. All IRMs must be placed in a "listening" mode before the synchronization signal is sent which sets the clock counter to zero. External data triggering and gating signals are also forwarded on to the IRMs through this additional "clock-board."

\subsection{LED calibration system}

In Section 4.5 I described the optical fibers that were connected to one end of the WLS fibers and guided scintillation light to PMTs. The other ends of the WLS fibers also protrude from the detector cube and are similarly coupled to clear optical fibers that then mount to twelve "LED pulser boards." Each of these boards powers 64 Lite-On (part number: LTL1CHTBK2) blue (peak wavelength: $468 \mathrm{~nm}$ ) LEDs (768 total) that can be set to fire light pulses into the fiber system, and thus the entire DAQ. A schematic of this system is shown in Fig. 4.14 and a photograph of a LED pulser board and its optical fibers is shown in Fig. 4.15. The frequency of these pulses is controlled by a NIM signal from a function generator and their intensity is set and adjusted by the DAQ computer. This system allowed 
calibration of the PMTs by studying the Poisson structure of low light pulses, see Sec. 6.1.2.

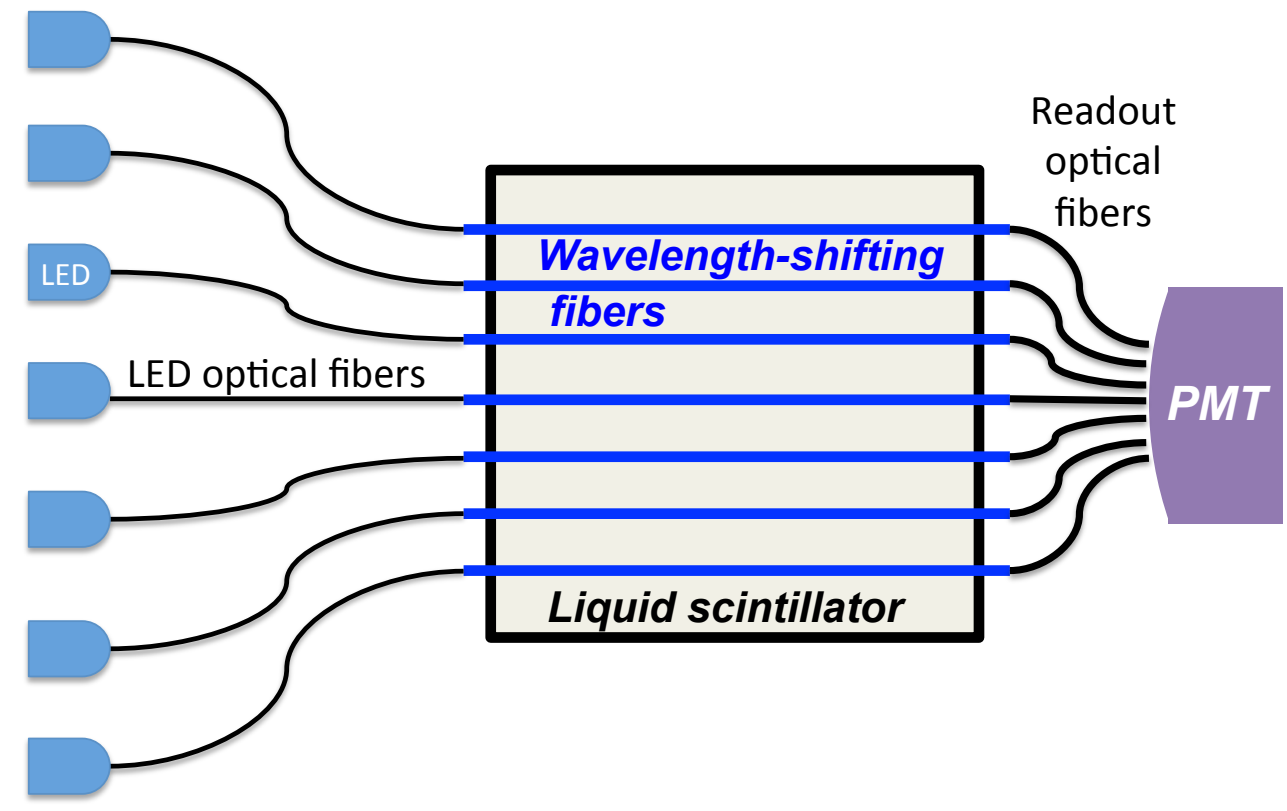

Figure 4.14: Schematic of the LED calibration system showing from left to right: pulsed LED system, LED end optical fibers, WLS fibers inside the detector cube, readout end optical fibers, and a PMT.

These pulser boards and their custom power supply were designed and built at Indiana University. The twelve boards mount directly to the frame of the detector and are all interconnected through a "daisy chain" of power, serial (for control signals), and NIM (LEMO, for triggering) cables. Since the LEDs are able to produce relatively high intensity light, smaller and more flexible $0.5 \mathrm{~mm}$ diameter St. Gobain BCF-98 optical fibers are sufficient to guide LED light to WLS fibers. Fiber connections are also not as particular as was required for the readout optical fibers. The fiber ends were fly cut with a diamond-edged cutter like the readout fibers, but are simply held against the LEDs on one end and the WLS fibers on the other without the use of optical grease. To hold the LED pulser optical fibers against the WLS fibers a hollow aluminum cylinder is used, similar to the cylinders used on the readout optical fibers. The cylinders on the LED side, however, have two different inner radii to match the $0.5 \mathrm{~mm}$ diameter of the clear optical to the $1.5 \mathrm{~mm}$ of the WLS fibers. 


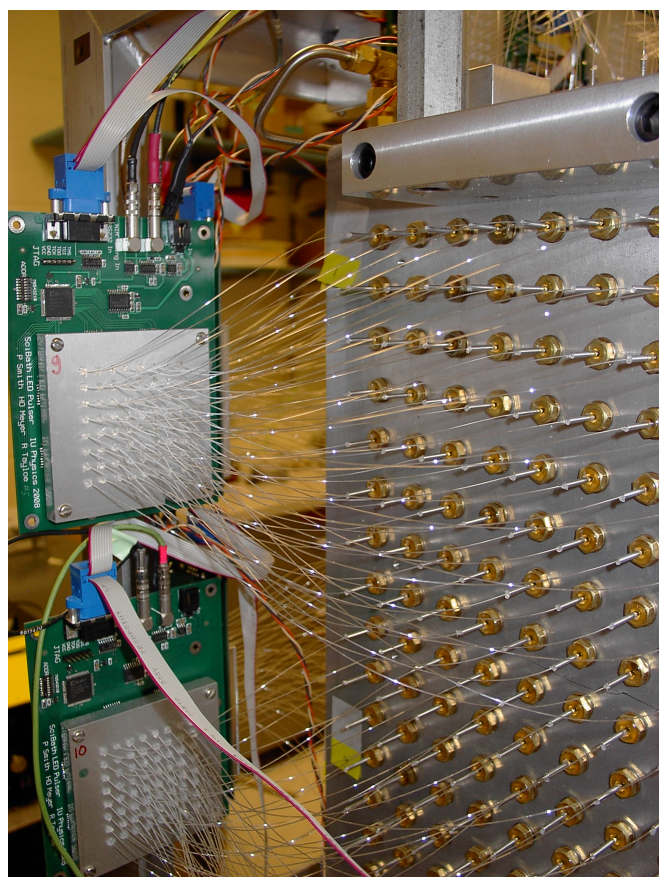

Figure 4.15: LED calibration system boards and their corresponding optical fibers.

The optical fibers were then held to the aluminum cylinder and the LEDs with a simple silicone adhesive. Four lengths of optical fiber were used in an arrangement to minimize physical strain on the fibers and their couplings.

\subsection{Plumbing}

SciBath has a relatively complex plumbing system that ensures the active liquid scintillator is pure, keeps the WLS fibers taut, and allows safe transport and storage of the scintillator. The system can be divided into two subsystems: liquid and gas. The entire plumbing system is diagramed in Fig. 4.16.

\subsubsection{Liquid scintillator plumbing}

The SciBath detector cube needs to remain full of clean scintillator during data-taking. This is accomplished by constantly pumping scintillator from the $103.5 \mathrm{~L}$ reservoir to the $81.7 \mathrm{~L}$ 


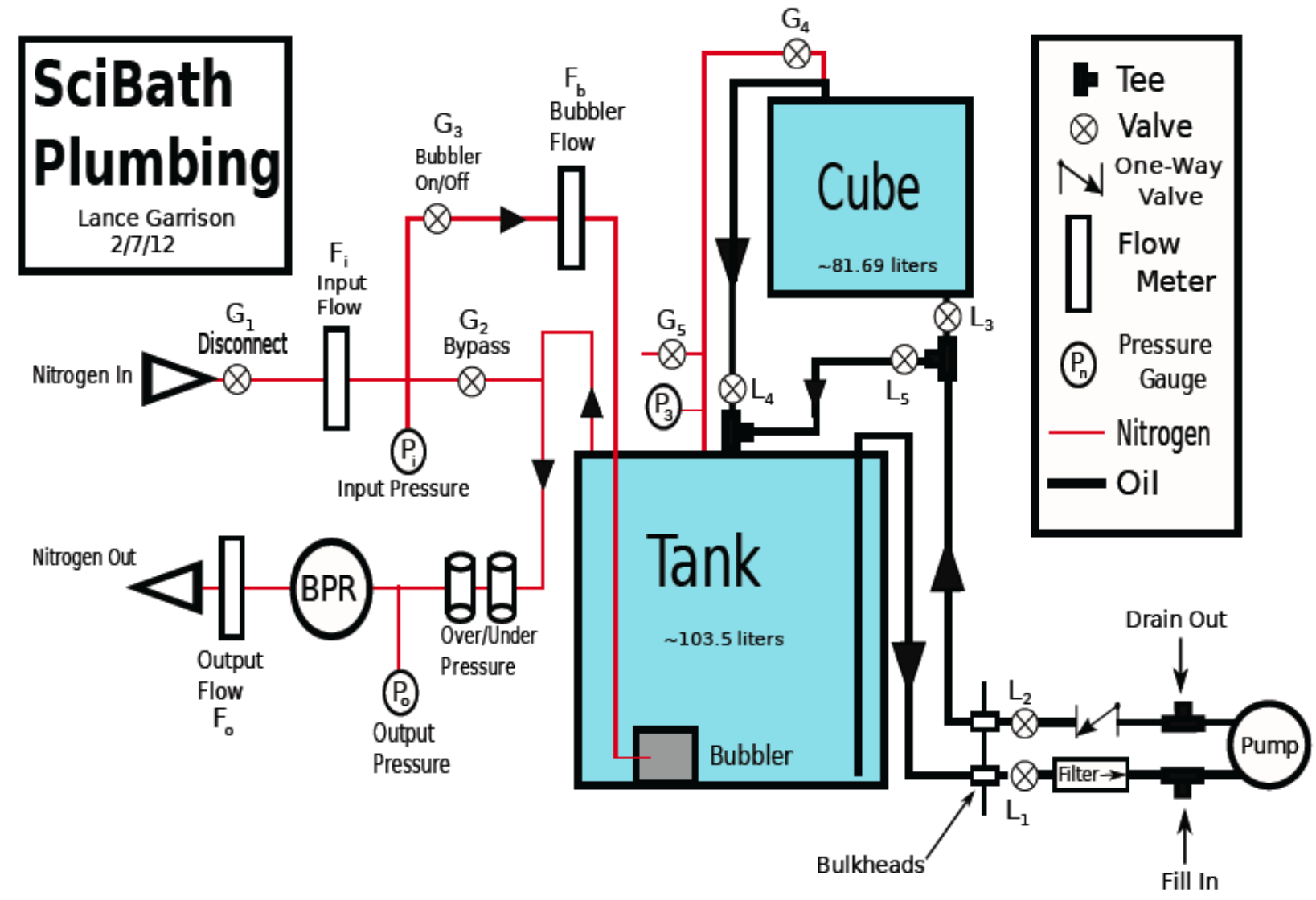

Figure 4.16: Schematic of the the SciBath nitrogen and liquid scintillator plumbing systems. 
detector cube. The scintillator is pumped into the bottom of the cube and excess is allowed to drain from the top of the cube back down to the reservoir tank. In this way scintillator is constantly, though slowly at about $0.3 \mathrm{~L} \mathrm{~min}^{-1}$, circulated between the reservoir and the cube. Along with ensuring that the cube is completely full of scintillator, this method also filters the scintillator during circulation.

The valves and connectors used for the plumbing system are primarily brass Swagelok [52] fittings. The current pump is a Fluid-o-Tech MGFR [53] pump with a $9 \mathrm{~mm}$ gear, but through the NuMI run an Autoclude model EV peristaltic pump was used. This pump was replaced because it slowly split its verderprene hoses and thus risked scintillator leakage. During the NuMI run these hoses had to be checked daily and replaced every week or two. It was suggested that pseudocumene in the scintillator was causing the hoses to swell and thus not seat properly in the pump, though this was not verified as the cause for the hose damage.

SciBath has between two and four levels of scintillator containment for possible leaks, depending to the location of the leak. Some seepage from the WLS fiber mounting pistons in the detector cube occurred during the NuMI run. This scintillator was directed to a jug inside the detector frame via a homemade gutter system. The bottom panels of the detector are liquid tight and of a volume such that the entire 103.5 L reservoir tank of scintillator can be contained. The entire detector is also placed inside of two New Pig [54] Collapse-A-Tainer spill containment systems, each of which can contain the full 103.5 L. Thus leaks from the detector cube have four levels of containment while leaks from the reservoir tank have three. The pump is mounted to the exterior of the detector, however, and therefore has two levels of leak containment.

The scintillator plumbing system can be configured for at least five modes of operation. During cube filling and data-taking all liquid valves except L5 are opened and the pump is on. During cube draining valves L1 and L2 are closed, valves L3, L4, and L5 are opened and the pump is powered off allowing the cube to drain by gravity alone. To add scintillator to 
the detector valves L1, L3, and L4 are closed, valves L2 and L5 are opened, and a pipe to the new scintillator is connected to the appropriate tee by the pump. To remove scintillator the same procedure is used except valves L1 and L2 are opened and closed, respectively, and the output pipe is connected to the other tee near the pump. For storage or transport all of the scintillator is drained into the reservoir and all of the liquid valves are closed.

\subsubsection{Nitrogen plumbing}

The SciBath nitrogen system has two purposes: to maintain a slight overpressure and to purge oxygen from the scintillator. The slight overpressure of roughly 1 psi causes the WLS fiber mounting pistons to slide slightly and thus pulls the WLS fibers taut, allowing the most accurate topological event reconstruction. Oxygen causes quenching in liquid scintillator and thus lowers the light output. This and other impurities are removed by bubbling nitrogen through the scintillator in the reservoir tank. This bubbling was done at the beginning of the NuMI run and since nitrogen was continually flowing over the scintillator during datataking, there was thought to be no opportunity for oxygen to enter the scintillator. In practice, however, some oxygen made it into the scintillator when peristaltic pump hoses were checked or replaced, though it is unclear exactly what effect this may have had.

Operation of the nitrogen system includes only two modes: bubbler on or bubbler off. To use the bubbler simply open valve G3 and close valve G2 with sufficient input pressure. Reversing these valves gives the standard data-taking mode where nitrogen flows over the scintillator but the bubbler is not used. As a safety measure, under- and over-pressure release valves were installed.

\subsection{Monitoring}

The SciBath remote monitoring system was developed to allow researchers to check in on the detector without needing to be physically present. This allows researchers to verify 
successful running and data collection as well as to ensure the detector presents no safety hazards. Throughout the NuMI run a researcher logged in to the SciBath DAQ computer using Virtual Network Computing (VNC) roughly every six hours and filled out an electronic monitoring log. Through this connection the researchers could access the run control GUI, Fig. 4.19, which gives the running status of the DAQ system as well as the most recent entires in the DAQ syslog. The diagnostic shown in Fig. 4.20 shows real time data characteristics including event rates, PE distributions, and errors. Figure 4.21 shows a similar diagnostic that is created at the end of each run and provides run summary statistics. To monitor the physical aspects of the detector, two web cameras were installed on the DAQ system local network. Example snapshots from these can be seen in Figs. 4.17 and 4.18 . These cameras allow monitoring of the level of scintillator in the reservoir tank, the nitrogen flow rates, the internal pressure, the pump activity, and the temperature and humidity at the detector location.

Running a liquid scintillator detector underground requires extra safety precautions. In particular, fire and scintillator leakage are very hazardous. A smoke detector was installed with an interlock to power down the detector in case of fire. The best way to check for scintillator leaks during running is to ensure the pump is always on (so the cube is always full) and closely monitor the level of scintillator in the reservoir tank. The reservoir level gauge is sensitive to about $0.1 \mathrm{~L}$, so a loss of $0.5 \mathrm{~L}$ of scintillator would be obvious. Additionally, the webcam in Fig. 4.17 should be positioned such that it can monitor the exterior leak containers. 


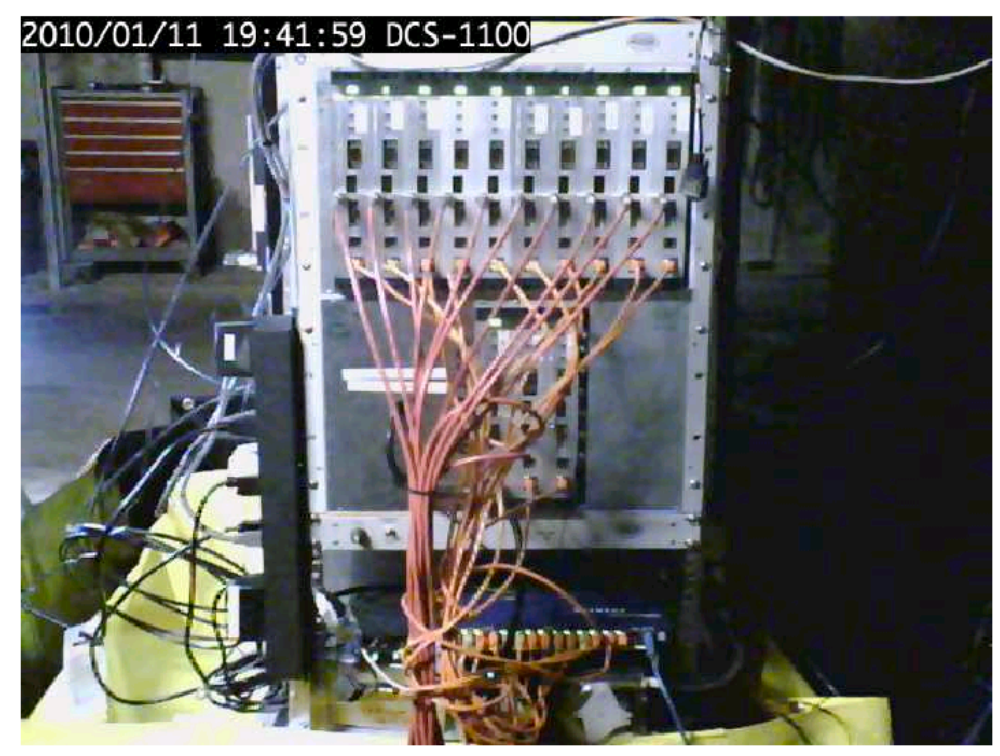

Figure 4.17: The view from one of two webcams with which SciBath can be monitored remotely. Two external leak containers can be seen underneath the detector (one yellow, one black).

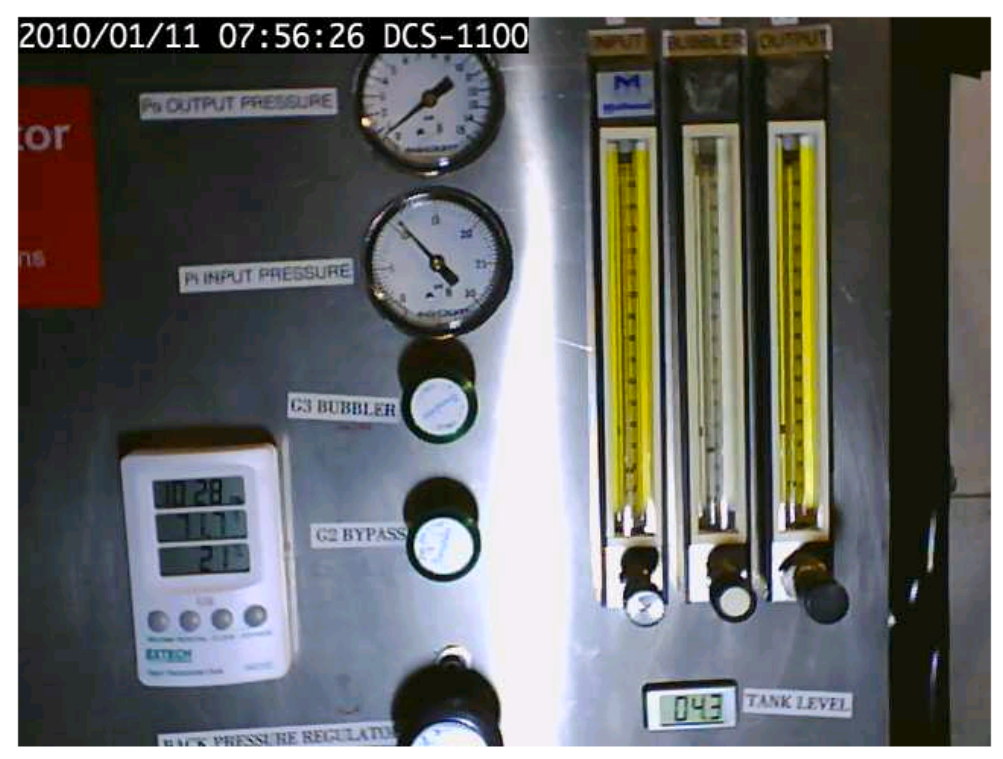

Figure 4.18: The view from one of two webcams with which SciBath can be monitored remotely. The two gauges at the top center give the internal pressure; the three gauges at the right give the $\mathrm{N}_{2}$ flow rates; the white gauge to the left gives the time, temperature, and humidity; and the digital gauge at the bottom right gives the scintillator level in the reservoir tank. 


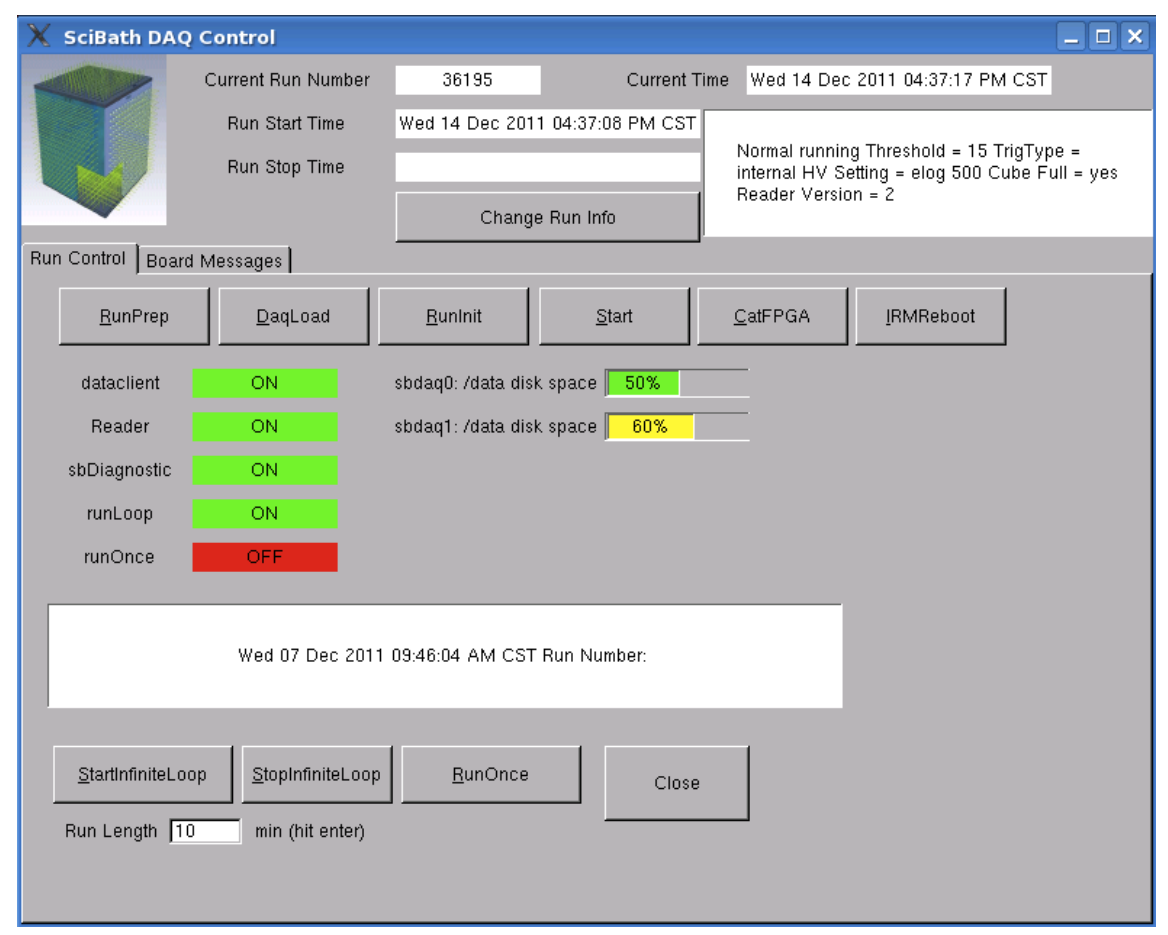

Figure 4.19: The SciBath run control GUI, which also gives the run status during monitoring. 


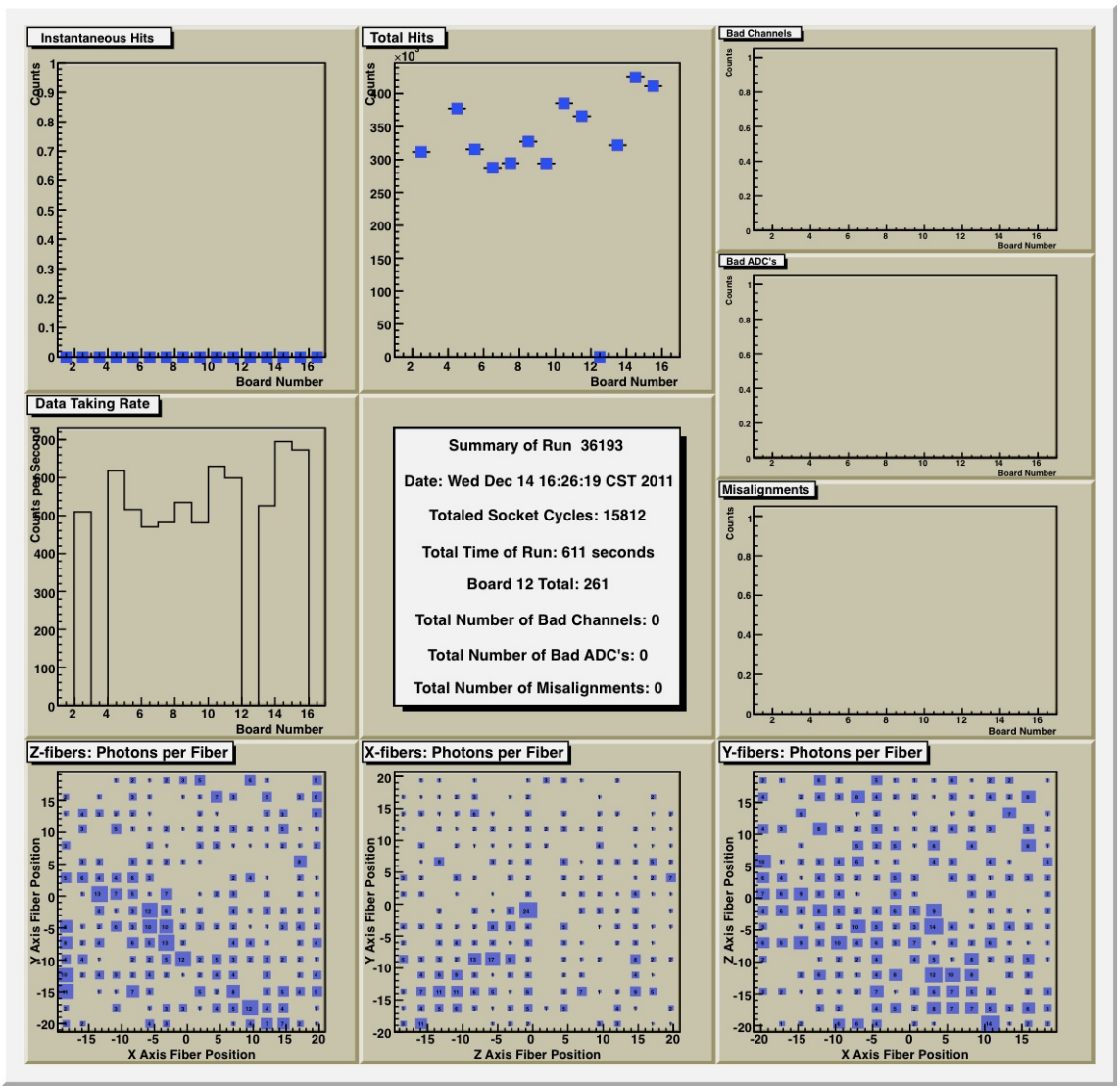

Figure 4.20: This diagnostic output for real time run monitoring provides run summary statistics including event rates (top and left), error reports (right), and fiber-by-fiber response summaries (bottom). 


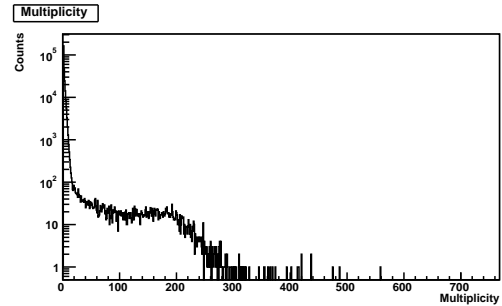

\section{Run Number: 42626 \\ Number of Events: 275781 \\ Number of Hits: 1051275}
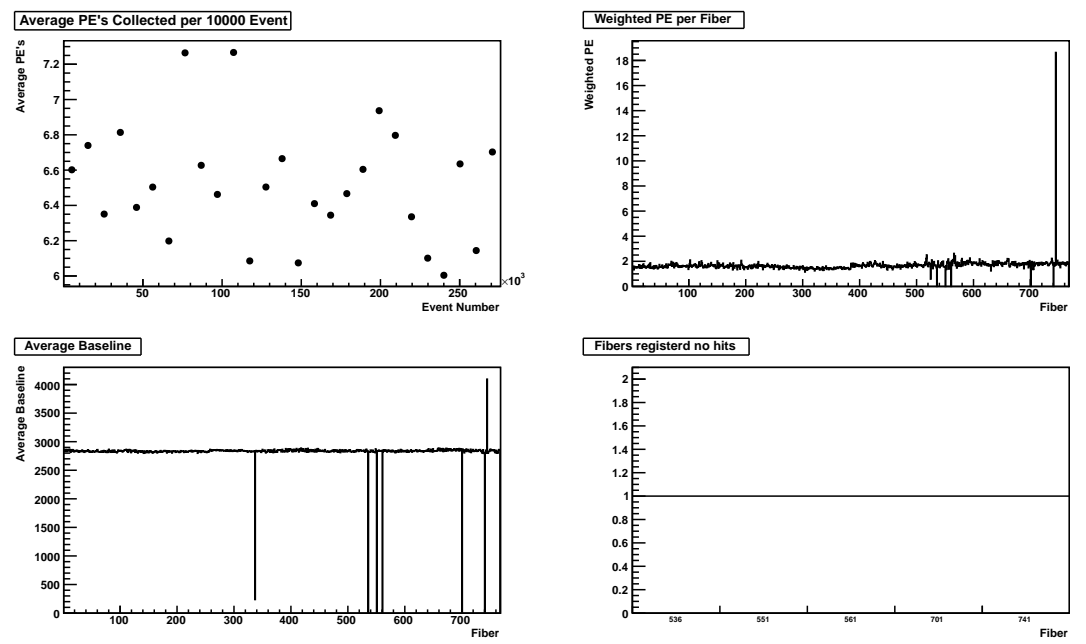

Figure 4.21: This run summary diagnostic provides PE, multiplicity, event count, and waveform baseline summaries. 


\section{Chapter 5}

\section{The NuMI Run}

The analysis I present in this thesis was based on data from the "NuMI run." This run was named after the Fermi National Accelerator Laboratory (FNAL) beamline on which SciBath was placed. In this chapter I describe the MINOS near detector hall, at which SciBath was located, as well as the basic characteristics of the NuMI neutrino beam. I also provide a summary of the beam output and describe the MI-12 run, which provided calibration and validation data for this analysis.

\subsection{The MINOS near detector hall}

The SciBath NuMI data was taken in the MINOS near detector hall at the Fermi National Accelerator Laboratory (FNAL). The hall was built to house the near detector for the Main Injector Neutrino Oscillation Search (MINOS) experiment, though it is also the site of the MINER $\nu \mathrm{A}$ detector. The hall is $100 \pm 5 \mathrm{~m}$ underground and along the Neutrinos at the Main Injector (NuMI) beamline about $1 \mathrm{~km}$ downstream of the beam target, see Fig. 5.1. This site therefore allows study of both beam-correlated and beam-uncorrelated particles. Figure 5.2 shows SciBath being lowered into the hall by crane. The surrounding bedrock is composed of dolomite and, to a lesser degree, shale and siltstone with a density of $2.43 \pm 0.19 \mathrm{~g} \mathrm{~cm}^{-3}$ depending on the specific location and groundwater content [55]. 
To more easily compare with other sites, the depth of the hall can be expressed in the units of meters water equivalent (m.w.e.). For standard rock with a flat overburden, the conversion is 2.65 m.w.e. per meter of rock. In these units the MINOS near detector hall is $265 \pm 27$ m.w.e., where the error is estimated based on the uncertainties in the rock density and depth.

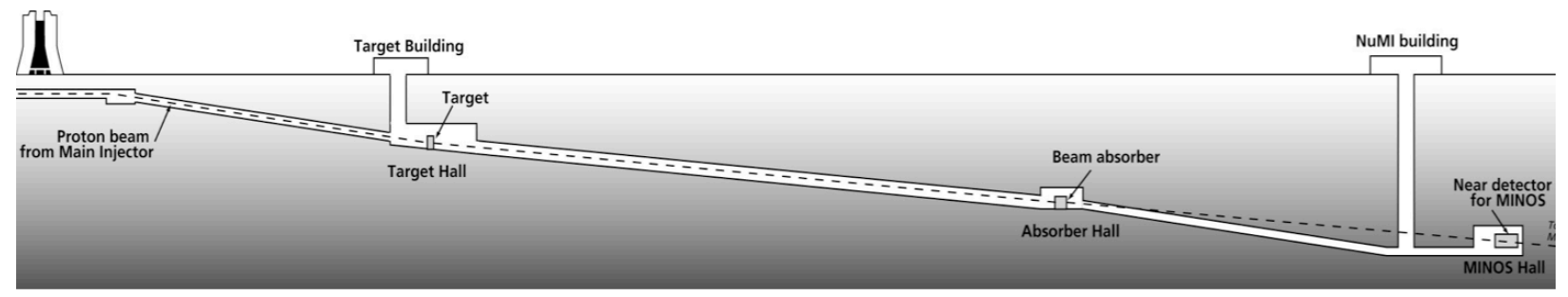

Figure 5.1: Side view of the NuMI beam line at FNAL. On the left side is the iconic Wilson Hall and on the right side is the MINOS hall, where SciBath was deployed. The dotted line represents the NuMI beamline.

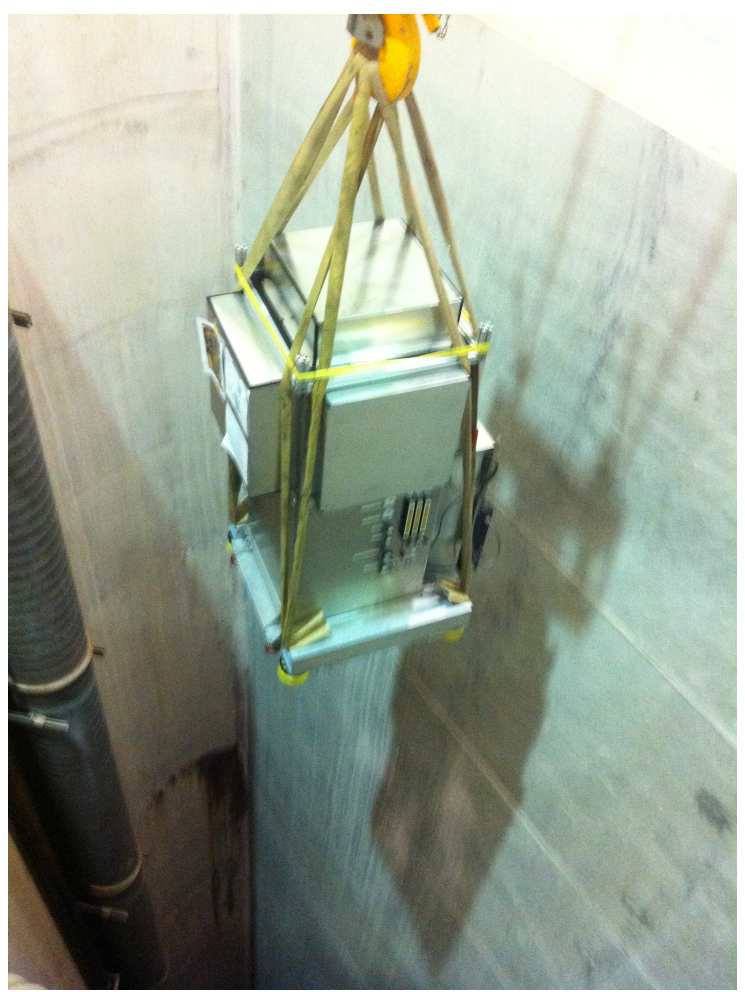

Figure 5.2: The SciBath detector being lowered to the MINOS near detector hall at FNAL. 


\subsection{The NuMI beam}

SciBath was around $5 \mathrm{mrad}$ off axis of the NuMI neutrino beam. A fraction of the protons from the FNAL Main Injector beam at $120 \mathrm{GeV}$ are fired onto a carbon target. This produces secondary mesons that are then focused by two magnetic horns. Neutrinos (and antineutrinos) are created by the leptonic decay of these mesons in flight along the $675 \mathrm{~m}$ decay pipe. A hadron absorber and $250 \mathrm{~m}$ of rock then absorb all particles of the beam except the neutrinos. Beam pulses were $10 \mu$ s wide and occurred every $2.1 \mathrm{~s}$ during normal operation. The internal time structure of the pulses is shown in Fig. 7.10. A trigger signal preceded each beam pulse by 214 ss, which was recorded along with the data. This allowed inclusion or exclusion of beam-correlated data during analysis. Throughout the NuMI run the beam was configured in low energy neutrino mode.

\subsection{NuMI run summary}

The SciBath NuMI run occurred during October, November, and December of 2011. The detector was loaded into a U-Haul truck, see Fig. 4.5, and moved to FNAL on October 3, 2011. Over the next two weeks the detector was installed in the MINOS near detector hall, see Fig. 5.2, and necessary training and safety approvals were acquired. Calibration running started on October 18, 2011 and continued through November 3. Production data was taken from November 4 through December 21, 2011. This data was recorded at a $0.5 \mathrm{PE}$ (per channel) threshold and resulted in a $6 \mathrm{kHz}$ singles rate. Data was taken continuously (24 hours a day, seven days a week), allowing collection of beam-correlated and beamuncorrelated data simultaneously. SciBath ran in this mode for seven weeks and collected a total of $6.5 \mathrm{~TB}$ of data. Initial estimates predicted around 400 neutrino events and 400 cosmic

neutron events, about 10 neutrons per day, in SciBath over this period. With the final cuts, 166 neutrons were counted in our target energy range. Neutrino analysis has not been done at this time. 


\subsubsection{NuMI beam output summary}

The output of the NuMI beam is measured by the number of protons that hit the carbon beam target, commonly called protons on target (POT). The effective total POT collected by SciBath during the NuMI run was estimated by adding POT recorded in the MINOS database for the relevant time period and scaling by the detector average live time efficiency. While operating, SciBath took a series of 10 minute runs, each live 100\% of the time. Some time was lost between runs, reducing the detector ideal live time efficiency to $95 \%$. In practice, the live time for a given day was possibly less than $95 \%$ of 24 hours due to calibration or erroneous runs. The effective live time efficiency was calculated for each day and multiplied by the beam output as recored in the MINOS database. Summing this product for the days of the NuMI run gave a total observed beam output of $5.21 \times 10^{19}$ POT. Figure 5.3 shows the total POT observed versus time for the NuMI run. After this analysis $2.29 \%$ of the data set was discarded due to further possible errors. These discarded runs were spread throughout the run period and thus the loss to live time and beam output observed was applied as a simple scale factor. Thus the final beam output observed was $(5.1 \pm 0.1) \times 10^{19}$ POT with an effective live time efficiency of $(87 \pm 2) \%$. The error was estimated based on the number of runs that could have been discarded twice: once when counting daily live times and again when when the additional $2.29 \%$ was removed.

\subsection{The MI-12 run}

Following the NuMI run SciBath took a separate measurement of neutrons at FNAL for a separate experiment. Neutron rates near the Booster Neutrino Beam (BNB) target in the MI-12 target building are a background for an off-axis, low-energy neutrino source to be used for CENNS, an experiment to measure of coherent neutrino scattering on liquid argon [56]. From March to May 2012, the detector was placed approximately $20 \mathrm{~m}$ behind the the BNB target in the MI-12 target building. The detector ran for about two months gated for $20 \mathrm{~ms}$ 


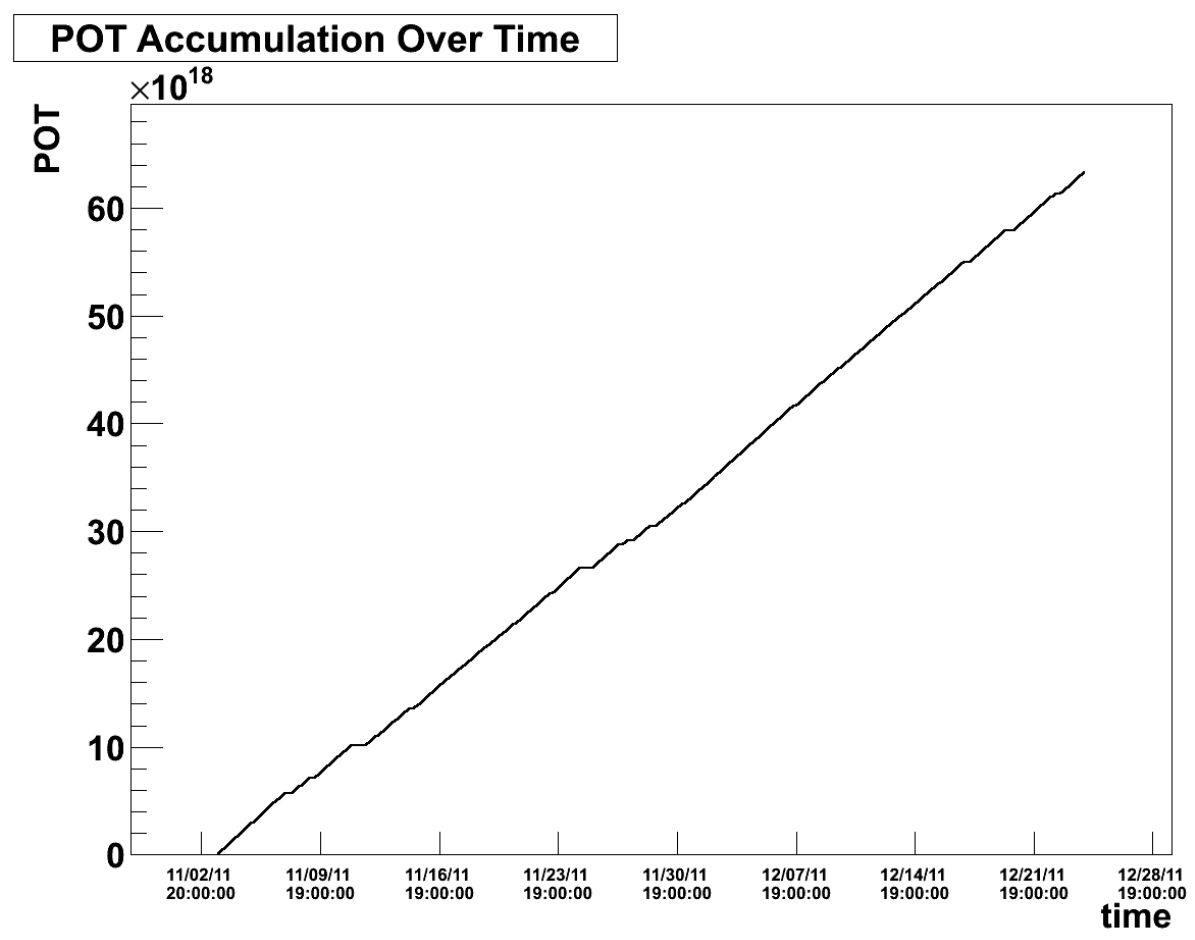

Figure 5.3: Cumulative protons on target (POT) versus time during the NuMI run.

for each beam pulse. The beam consisted of approximately six $1.6 \mu$ s micro-pulses at a repeating rate of $15 \mathrm{~s}^{-1}$ per macro-pulse, and each macro-pulse came approximately once every $2.1 \mathrm{~s}$. Improvements in the DAQ allowed SciBath to record over $95 \%$ of all beam pulses in the 5.5 week production data run. The total POT for production runs was $3.50 \times 10^{19}$ and an additional $4.08 \times 10^{18}$ were recorded during a one week beam off-target systematics check. Because data collection was gated, the detection threshold was lowered to 10 ADC channels per fiber which is nominally $1 / 3 \mathrm{PE}$. At this threshold, the total event rate was approximately $10 \times 10^{3} \mathrm{~s}^{-1}$ which yielded $2.5 \mathrm{~TB}$ of total data. 


\section{Chapter 6}

\section{Analysis Methodology}

A great deal of analysis is required to uncover the physical measurements hidden in the large raw data sets of particle physics experiments. Indeed the vast majority of the data recorded during the NuMI run was the result of physical processes in which we have no interest: background particle interactions, PMT noise, electronics noise, etc. This chapter describes the analysis that allowed us to separate the target data from the background data and interpret it as a physical measurement.

In the first section I describe how SciBath events were constructed. In the next three sections I discuss how we were able to use event time, energy, and topology to identify candidate muon and neutron events; though as with most particle physics experiments, some background events were misidentified as muon and neutron events. In Section 6.6 I describe how a background subtraction was performed to remove the number and spectra contributions of the misidentified events, leaving only the number and spectra of target interactions. In the final section I describe the Monte Carlo simulation that was used to interpret the final event counts as physical measurements. 


\subsection{Event building}

The SciBath readout electronics output data as a series of time ordered PMT hits. In this section I describe the analysis that was done to interpret these hits as "events," which are intended to represent a single particle interaction or a series of interactions that occur rapidly in time, within $300 \mathrm{~ns}$. In the first subsection I describe how PMT waveforms were fit to accurately identify the hit time and amplitude. In the second subsection I describe the analysis that determined how hit amplitude related to the number of photoelectrons (PEs) released on the photocathode, which corresponds to the number of scintillation photons captured by a single WLS fiber inside the detector cube. In the third and fourth subsections I then describe how a minimum number of PMT hits were grouped by time into events.

\subsubsection{Waveform fitting}

A waveform is a set of analog-to-digital converter samples that reproduce a single photomulti-

plier tube (PMT) pulse. Sample raw waveforms from SciBath can be seen in Fig. 4.13. Analysis of these waveforms provides the reconstructed time and integrated amplitude (charge) of a PMT pulse. The "ringing oscillator" shaper circuits on the SciBath readout electronics give waveforms the form

$$
f(t)=-A \exp \left(\frac{t-t_{0}}{\tau}\right) \sin \left(\omega\left(t-t_{0}\right)\right)+B, \quad \text { for } t>t_{0} .
$$

Here $A$ is a scale parameter that depends on the amplitude of the PMT pulse, $t_{0}$ is the start time for the pulse, $\tau$ and $\omega$ are parameters set by the readout circuit, and $B$ is the ADC level when no pulse is present.

A waveform fitting analysis was applied to all PMT pulses, also known as "hits," that were included in the production data set. This procedure, optimized for accuracy and computation time, provided a hit time and amplitude for each PMT pulse with resolutions 5 ns and 0.5 PEs, respectively. In practice, a parabolic fit function was used to fit the waveform 
in the region of the minimum ADC value instead of the full function (Eqn. 6.1). This simplification allowed for an analytical solution instead of a numerical fit, saving copious CPU-time. Once a fit was performed, the time and ADC value of the minimum of the PMT pulse were calculated as

$$
t_{\text {min }}=-\frac{b}{2 a}, \quad A D C_{\text {min }}=a t_{\text {min }}^{2}+b t_{\text {min }}+c,
$$

using the solution from the fitted parabola of the form $a t^{2}+b t+c$. The hit amplitude (in units of ADC channels) was then calculated as

$$
\text { hit amplitude }=B-A D C_{\min } \text {, }
$$

where the baseline $B$ was simply the average ADC value of the samples before the PMT pulse, the first five readings in Fig. 4.13 . The start time of the pulse, $t_{0}$, was calculated based on the form of Eqn. 6.1 as

$$
t_{0}=t_{m i n}-\frac{\arctan (\tau \omega)}{\omega} .
$$

This result can be easily derived by requiring the minimum Eqn. 6.1 to occur at $t_{\min }$. The parameters $\tau$ and $\omega$ were found by fitting thousands of waveforms with Eqn. 6.1 and taking the mean of their $\tau$ and $\omega$ distributions. The $\chi^{2}$ test was used with Eqn. 6.1 to calculate a quality-of-fit value. Sample waveforms displaying relevant fit results can be seen in Fig. 6.1.

Further details regarding the waveform fitting method can be found in Appendix C.

\subsubsection{Photoelectron calibration}

An ideal photomultiplier tube (PMT) produces discrete voltage output according to the number of photoelectrons (PEs) generated on the photocathode. Unfortunately, readout electronics do not measure the number of PEs directly because a signal of just a few electrons 

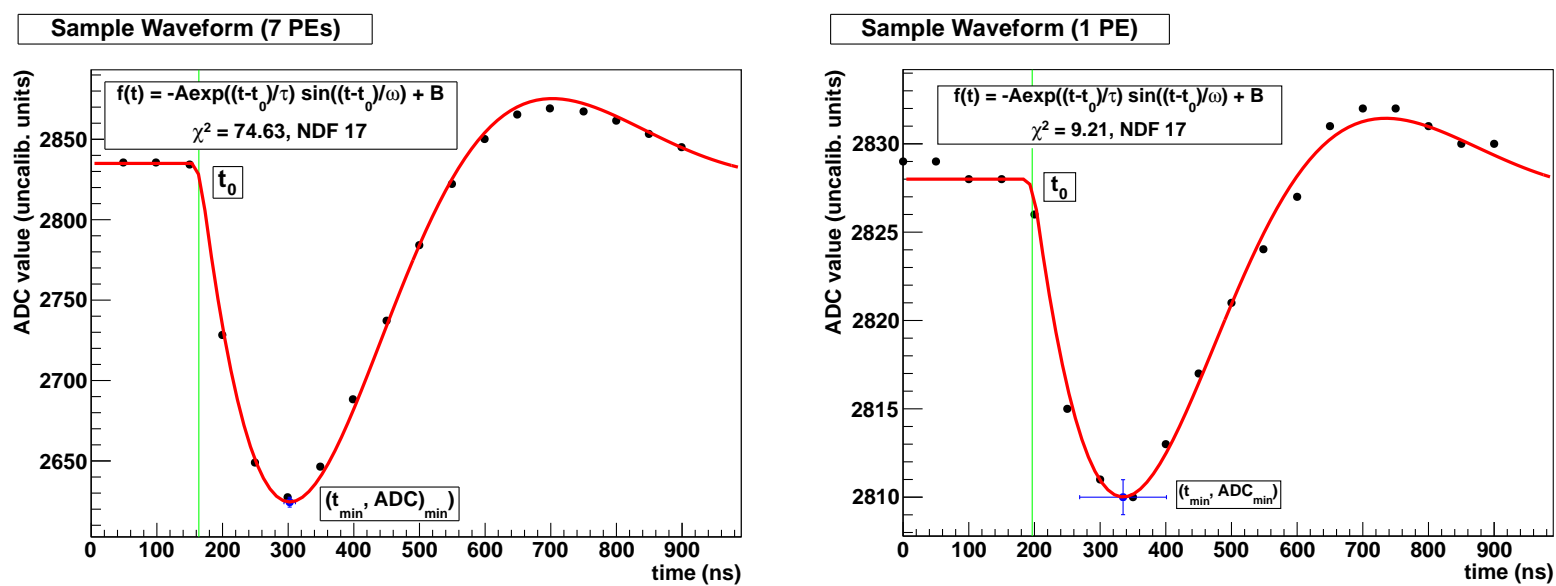

Figure 6.1: Examples of waveform fitting. The red curve shows a fit with Eqn. 6.1. The green line indicates the fitted start time and the blue point indicates the minimum of the pulse, which was used to calculate the pulse amplitude.

is too weak. Instead the PEs create an avalanche of electrons inside a multiplier tube and the integrated charge of this signal is digitized by the DAQ through an Analog-toDigital-Converter (ADC). Thus, the fundamental unit of scintillation detectors' response is integrated charge, measured in ADC channels. Physically, the number of PEs is a better unit for detector response because it is not altered by the statistical variation in PMT gain. A method was generated to reconstruct the number of PEs at the photocathode as a function of the size of the PMT pulse as measured in ADC channels.

As is typical of particle interaction counting, the distribution of PEs produced from a mono-energetic light source is Poisson. In any real photomultiplier tube, however, the output for any (discrete) number of PEs is smeared by statistical variations in photomulitplier gain, assumed to be Gaussian distributed. With this in mind, signal response (in ADC channels) was calibrated to PEs by pulsing each of the 768 SciBath PMT channels around two thousand times with the SciBath LED calibration system, described in Sec.4.7, and fitting the resulting output with a Gaussian-smeared Poisson distribution with 3 parameters: the Poisson mean, a scale factor for the standard deviation for gaussian smearing of all PE response greater than zero, and the ADC-to-PE calibration factor which quantified the calibration. Figure 6.2 
provides a pair of sample fits. The light output of the LEDs was set to generate a mean response between 0.7 and 2.3 PEs to allow a statistically significant response that shows the Poisson structure. If the PMT response were higher the output would look more Gaussian and the degeneracy between the mean PE response and the calibration factor (ADC response per PE) would be impossible to resolve.

Each PMT was gain matched by adjusting the PMT high-voltage between $750 \mathrm{~V}$ and $850 \mathrm{~V}$ until this fit method resulted in $30 \mathrm{ADC}$ channels in the DAQ, corresponding to the 1 PE peak, on average over all 64 channels. Variation of this calibration factor channelto-channel across each PMT was assessed with the same method. This individual PMT channel calibration was performed at Indiana University before taking data at FNAL and twice during data-taking at FNAL. Unfortunately we had problems with the LED calibration system power supply immediately after the move to FNAL, so the first PE calibration at FNAL was performed two months into the three month run. The second calibration at FNAL was performed at the end of the run, immediately after data-taking ended. Over the course of the data run gains were found to be stable to within $10 \%$.

\subsubsection{Event grouping}

The output from the SciBath readout electronics (IRMs) was simply a time ordered list of PMT hits, each of which consisted of a timestamp and a waveform (with extracted time and integrated charge). In order to reconstruct the particle interactions that generated these hits, they were grouped into "events," defined as a collection of hits that are expected to result from a single particle interaction. The event grouping process is illustrated in Fig. 6.3. The earliest hit of the run started the first event. All hits less than 300 ns after this first hit were grouped into the first event. The first hit to occur after this $300 \mathrm{~ns}$ window started a new event and the event building process repeated. In this way, generally all hits that occurred within a 300 ns window were grouped into an event. The 300 ns group timing was chosen to maximize the likelihood that all of the hits related to a particle interaction would 


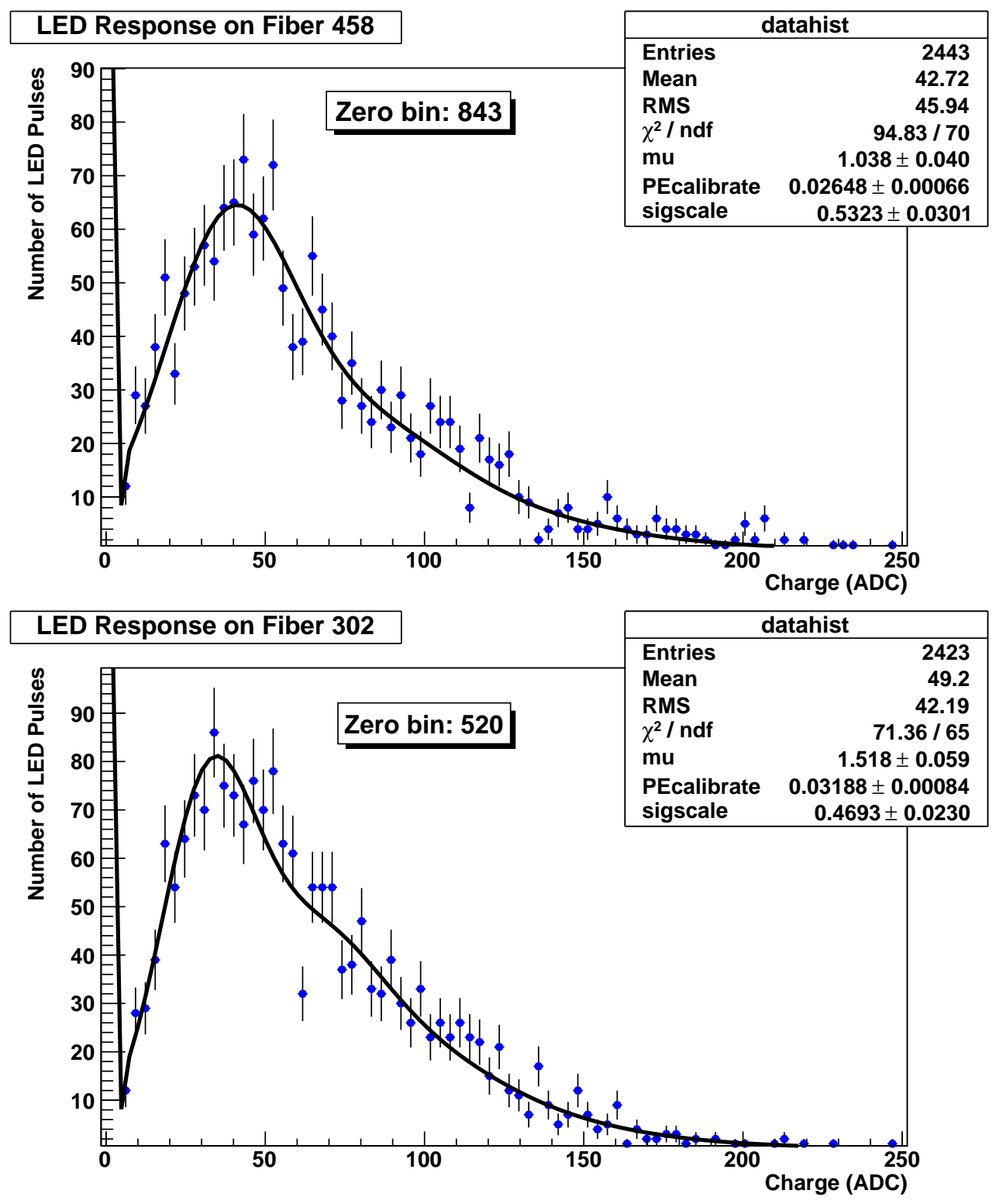

Figure 6.2: Histograms of the number of LED pulses versus hit charge as measured in analog-to-digital converter channels. The curve is a Gaussian-smeared Poisson function with Poisson mean "mu" in photoelectrons (PEs) and Gaussian sigma equal to "sigscale" times the number of PEs. The peaks at $40 \mathrm{ADC}$ channels are the one-PE response. A potential two-PE response is barely visible in the lower plot at $75 \mathrm{ADC}$ channels. 
be collected in one event while also minimizing the number of particle interactions that were divided into two events, as shown by events 3 and 4 in Fig. 6.3. The time of an event was defined as the time of its earliest hit and the charge of an event was defined as the sum of the charges of its hits.

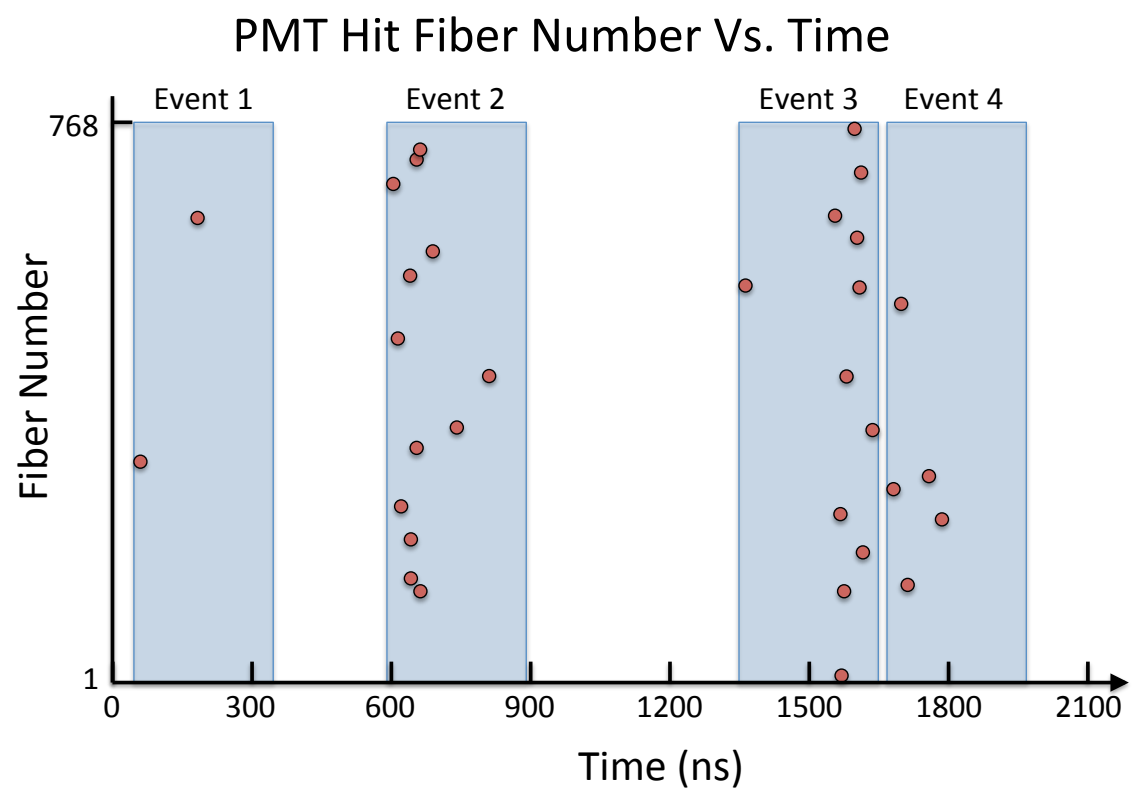

Figure 6.3: Diagram of the event building process. The first hit opens a $300 \mathrm{~ns}$ time window and all hits within are grouped into the first event. The first hit to occur after this window opens its own time window for the second event, and the process repeats. Event 1 is likely caused by PMT noise. Event 2 represents a typical event in SciBath. Events 3 and 4 show how a random PMT noise hit preceding a particle interaction can split the resulting hits into two separate events. The PMT noise rate is such that event splitting is rare; it is shown here simply to illustrate the event grouping method.

\subsubsection{Multiplicity}

Multiplicity is defined as the number of PMT hits grouped into a given event. It is strongly correlated with the energy deposited in the detector, as shown in Fig. 6.4. For the most part, this parameter has not been crucial to the analysis due to its redundancy with event energy. One exception is the cut of multiplicity greater than or equal to six that was applied. This cut removes very low energy events from our analysis, but it also removes events generated 
by PMT noise and reduces the data set to a more manageable size. As examples, Events 1, 2, 3, and 4 in Fig. 6.3 have multiplicities of 2, 13, 12, and 5, respectively. Therefore events 1 and 4 would would fail the multiplicity cut and be excluded from this analysis.

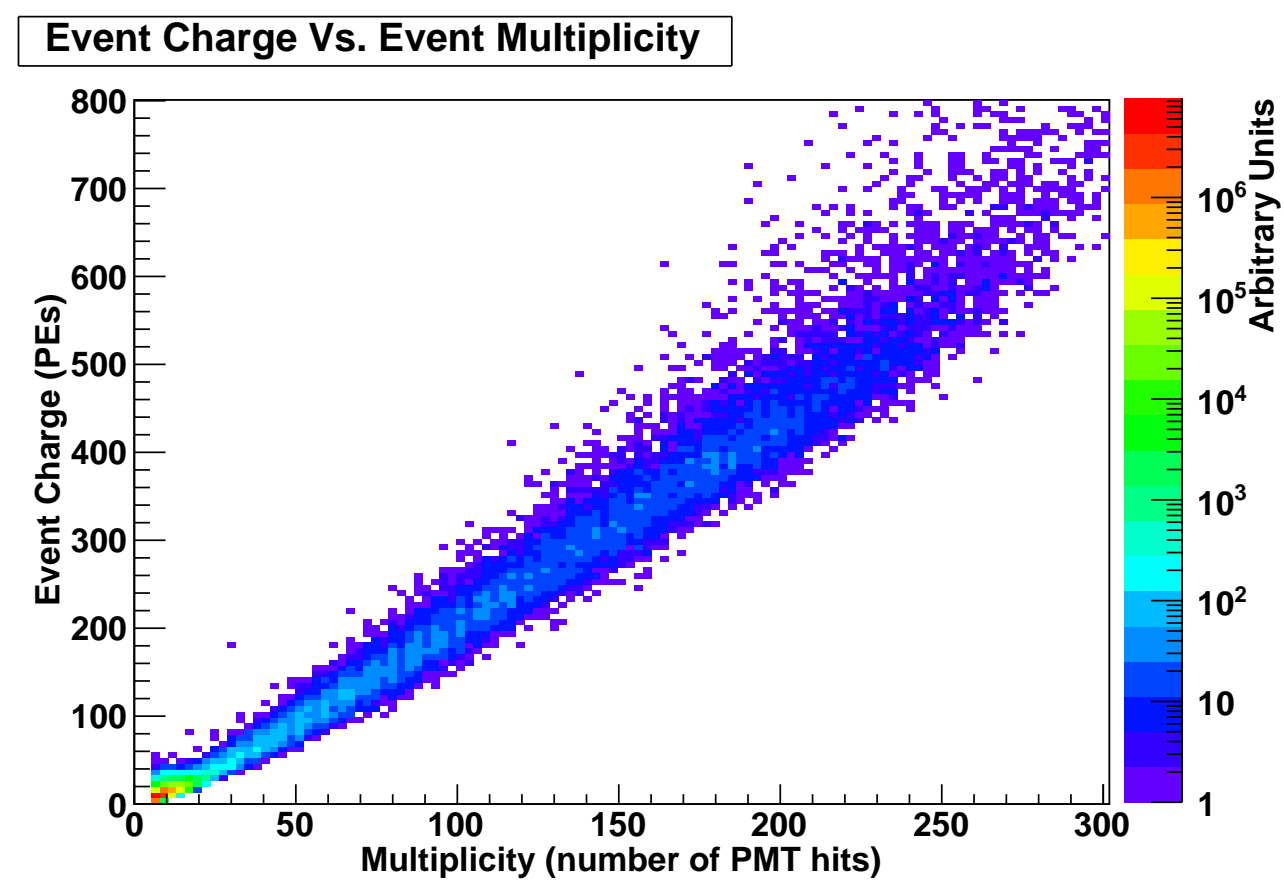

Figure 6.4: Event energy versus event multiplicity for $1 / 40$ of the NuMI data set. The linearity indicates a strong correlation of roughly 2 PEs per PMT hit, on average.

\subsection{Timing analysis}

In Sec. 2.4.4 I motivated neutron identification and reconstruction by looking for separate scattering and (delayed) capture events. Any such correlation analysis must pay close attention to the timing of events.

Individual PMT hits are labeled with a timestamp from the IRMs, see Sec. 4.6.5, with 50 ns precision and a timestamp from the DAQ computer with $1 \mathrm{~s}$ precision. The IRM timestamp is much more precise, but its clock resets after $214 \mathrm{~s}$, less than the NuMI default run time of $10 \mathrm{~min}$. The DAQ timestamp is required to sort events greater than 214 second apart and provide global timing. Once a hit waveform was fit its time was adjusted according 
to the fitted start time of the PMT pulse. The time of an event was then defined as the lowest hit time of any of the hits in that event. Break-in-board signals were assigned timestamps in the same manner, though they were not grouped into events. The first and last $5 \mathrm{~s}$ of each run were cut as IRMs may have been starting or stopping data-taking during this period causing incomplete detector response.

\subsubsection{Cosmic event timing}

One of the most basic cuts in the cosmic neutron analysis was the removal of events that could have been correlated to the NuMI beam. This was done by requiring the time difference between a cosmic neutron candidate event and the most recent beam trigger (214 $\mu$ s before a beam spill) to be greater than $2 \mathrm{~ms}$. Correlated events were identified as primary neutron or muon candidate events followed by neutron capture candidate events within a 200 us time window. A schematic of the cosmic timing cuts is shown in Fig. 6.5.

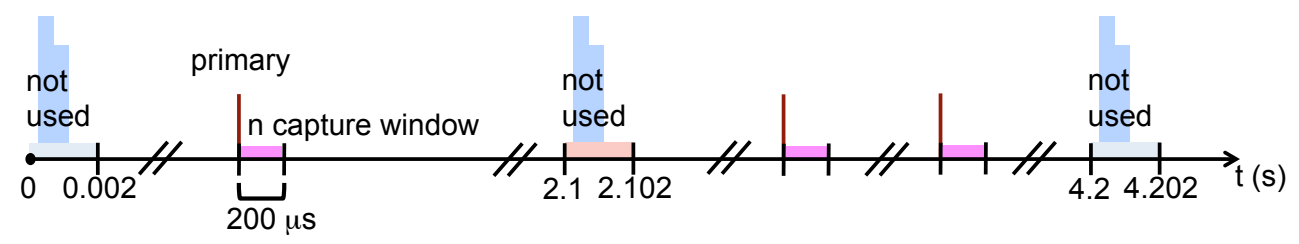

Figure 6.5: Timing analysis for cosmogenic neutron capture correlated events. An event well after any NuMI beam pulse that passes energy and topology cuts was considered a cosmic neutron or muon primary candidate and opened a $200 \mu \mathrm{s}$ window to search for neutron capture candidate events.

\subsubsection{Beam correlated event timing}

The beam correlated neutron analysis required neutron scattering (primary) events to occur within the 10 us beam window. Correlated neutron capture (secondary) candidate events were then required to occur in a $450 \mu \mathrm{s}$ window following the beam window, see Fig. 6.6. In reference to the time of the most recent beam trigger, scattering candidates were required 
to occur between $213 \mu \mathrm{s}$ and $224 \mu \mathrm{s}$ and all capture candidates to occur between $229 \mu \mathrm{s}$ and $663 \mu \mathrm{s}$. Additionally, the time difference between a neutron primary and its correlated secondary, $\Delta \mathrm{T}$, was required to be between $16 \mu \mathrm{s}$ and $450 \mu \mathrm{s}$. This time difference cut cleans up the $\Delta \mathrm{T}$ spectrum by removing the "ramping" structure cause by primary events that occur later (earlier) in the beam window having more phase space for shorter (longer) $\Delta \mathrm{Ts}$ than primary events that occur earlier (later) in the beam window.

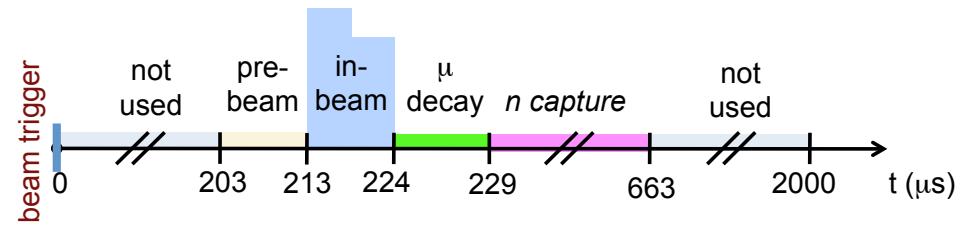

Figure 6.6: Timing analysis for beam-correlated neutron events. An event during a NuMI beam pulse that passes energy and topology cuts must be followed by a neutron capture candidate in a 450 us window ("n capture") that starts 5 us after the beam pulse.

\subsubsection{Hit time average and $\sigma$ cuts}

Each event is composed of multiple (at least six) hits, each of which with its own time. The average and standard deviation $(\sigma)$ of these hit times were calculated for every event, see Figs. 6.7 and 6.8. To remove erroneous events, neutron and muon primary candidate events were required to have a hit time standard deviation less than 100 ns. Neutron capture candidate events were required to have a hit time average and standard deviation less than 40 ns. This relatively tight cut on the neutron capture events substantially reduced their background.

\subsection{Energy analysis}

The amount of energy deposited in the detector, as interpreted by the number of PEs detected by the PMTs, is a powerful indicator of the type of particle interaction that created an event. 


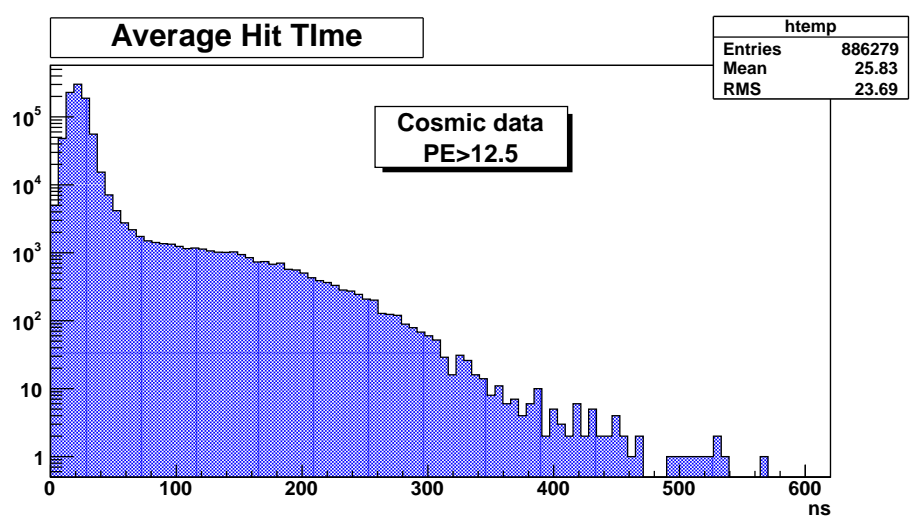

Figure 6.7: Distribution of average hit time within an event for all cosmic data that deposited more than 12.5 PEs of energy. Neutron capture event selection required average time less than 40 ns.

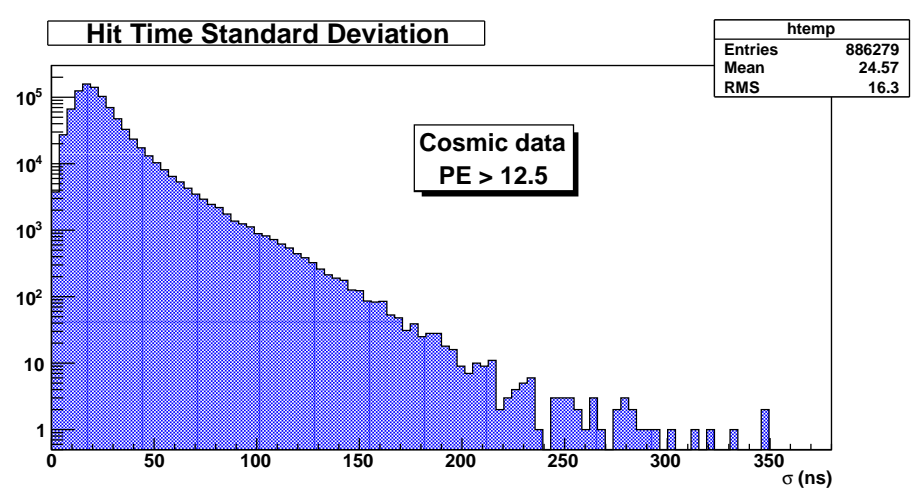

Figure 6.8: Distribution of hit time standard deviation within an event for all cosmic data that deposited more than 12.5 PEs of energy. Neutron scatter and muon event selection required $\sigma$ less than 100 ns while neutron capture event selection required $\sigma$ less than 40 ns. 
The specific energy cuts for the measurements of this analysis are listed in Table 6.1. In this section I go beyond the event identification cuts to describe how we calibrated energy deposits as measured in PEs to the more recognized units of MeVs. In the case of neutron elastic scattering events, this required special consideration of the known effects of proton quenching, which I discuss in the second subsection.

\subsubsection{Calibration based on minimum ionizing muons}

At the end of the event building process, Sec. 6.1, only an indirect measure of event energy was known: the total photoelectrons collected. Simulation was used to reconstruct the deposited energy for events based on their photoelectron response and the type of interaction expected.

Using the Geant4 Monte Carlo simulation described in Sec. 6.7, cosmic muons were simulated and the average energy deposited was found to be $82 \mathrm{MeV}$. Using the measured NuMI cosmic photoelectron response spectrum in Fig. 6.9, the peak at 420 photoelectrons was identified as the response to minimum ionizing muons. The SciBath energy response was calibrated based on this comparison as $82 / 420=5.1 \mathrm{MeV} /$ photoelectron.

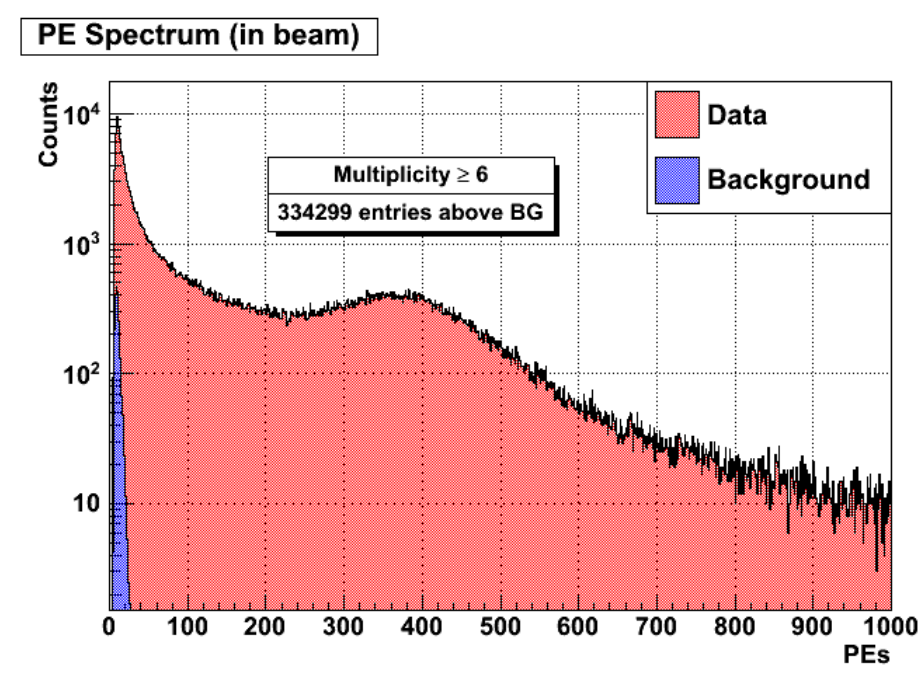

Figure 6.9: Photoelectron spectrum for all events in the NuMI beam. Minimum ionizing muons are responsible for the peak at 400 PEs. 


\subsubsection{Quenching of proton response}

Naively one may assume the light output of scintillator to be directly proportional to the amount of energy deposited. Quenching in scintillator describes the observed nonlinearity of light output from a charged particle track as a function of particle type and stopping power, $d E / d x$ [21]. The $2.2 \mathrm{MeV}$ photon resulting from neutron capture interacts with scintillator by Compton scattering. The recoiling electron from Compton scattered generates a detectable scintillation signal. Conveniently, quenching of electrons can be ignored in this analysis because it is only observed at energies below $125 \mathrm{keV}$ [21], below our threshold. Quenching must be considered, however, to reconstruct the energies of recoiling protons from neutron elastic scattering, our primary neutron signal.

The characterization of proton quenching in liquid scintillator as measured by Braizinha et al. [57] was used for this analysis. They measured the light output for four scintillators as a function of proton energy and fit the data with Birks' law [58] plus an additional quadratic term suggested by Chou [59]. The form of the fit is:

$$
L(E)=\int_{0}^{E} \mathrm{~d} \epsilon\left[1+k B\left(\frac{\mathrm{d} E}{\mathrm{~d} x}\right)+C\left(\frac{\mathrm{d} E}{\mathrm{~d} x}\right)^{2}\right]^{-1}
$$

Braizinha et al. reported values $k B=9.2 \times 10^{-3} \mathrm{~g} \mathrm{~cm}^{-2} \mathrm{MeV}^{-1}$ and $C=1.6 \times 10^{-6} \mathrm{~g}^{2} \mathrm{~cm}^{-4} \mathrm{MeV}^{-2}$ from their fit to the data in their Table 1 for scintillator BC-517H, which is similar to the SciBath scintillator. Using the NIST PSTAR [60] to generate values for the proton stopping power as a function of energy, the energy calibration described in Sec. 6.3.1, and Eqn. 6.5, the following conversion from photoelectron response $(P E)$ to proton energy deposited $(E)$ was generated:

$$
E(P E)=7.766+0.27953(P E)^{0.96}
$$

This energy calibration was used for neutron scattering candidate (primary) events. All other events were calibrated in energy as described in Sec. 6.3.1. 


\subsection{Topology analysis}

The SciBath detector was designed for fine-grained topological analysis. Particle interactions are reconstructed based on light guided by a three dimensional grid of optical fibers, see Fig. 4.4. In this arrangement each fiber hit provides a two dimensional position reconstruction and the full grid is easily visualized as three two dimensional projections, as shown in the event displays in Fig. 6.10. The fibers have been categorized by the dimension with which they are aligned: $\mathrm{x}$-fibers are those that run parallel to the $\mathrm{x}$-axis, $\mathrm{y}$-fibers are those that run parallel to the y-axis, etc. See Fig. 4.3 for the definition of the SciBath coordinate system.

In this section I describe the analysis methods used for event identification based on the SciBath topology. I first describe event cuts requiring energy response in three dimensions and event positions within fiducial volumes. I then give an overview of the eigenvalue analysis that was used to reconstruct particle tracks and to separate muon and neutron events.

\subsubsection{Required energy response in three dimensions}

Since each fiber runs across the length of the detector, each parallel set of fibers can only report position information for the two dimensions that are perpendicular to those fibers. For example, an x-fiber hit is located at a particular $\mathrm{y}$ and $\mathrm{z}$ location (the position of the fiber), but could have originated at any $\mathrm{x}$ because the fiber spans all of $\mathrm{x}$. To be able to reconstruct a particle interaction in three dimensions at least two sets of fibers were required to contain a hit. Looking at a SciBath event display, Fig. 6.10, this cut requires at least two

of the three projections to show a response. This cut was applied to all data in this analysis, but was significant only for lower energy interactions. 

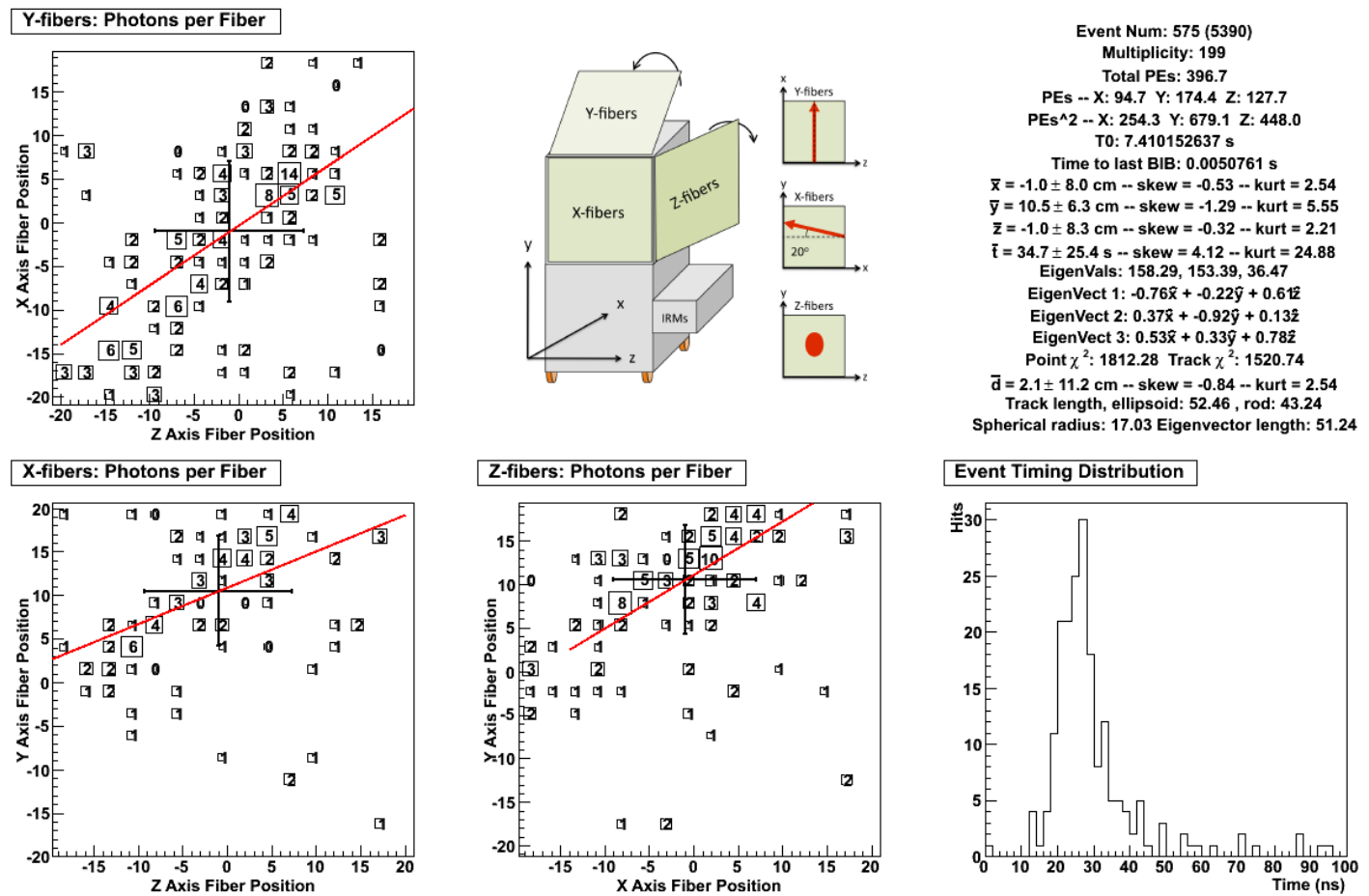

(a) Event display for a muon candidate event.
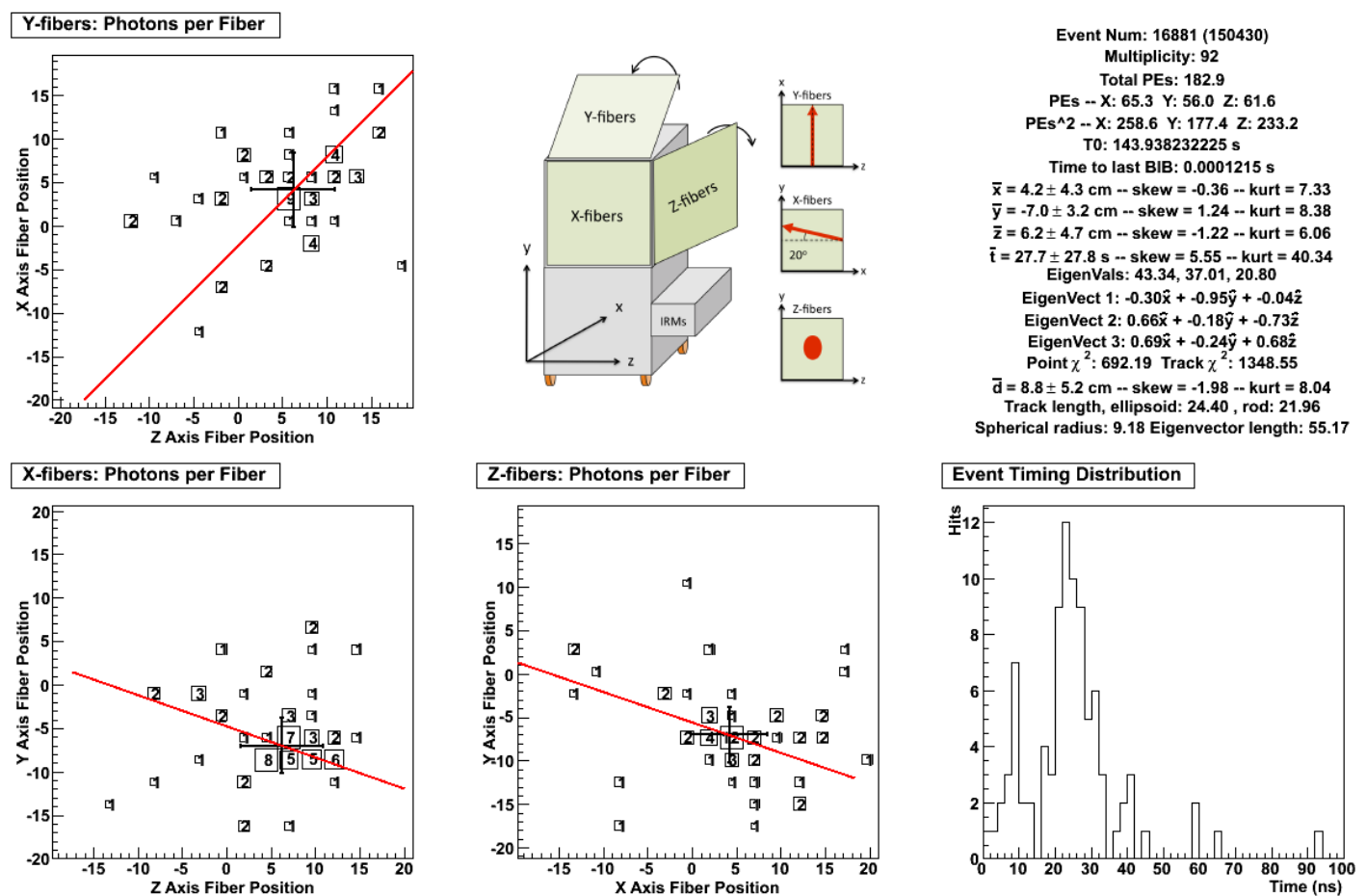

(b) Event display for a neutron scattering candidate event.

Figure 6.10: Sample SciBath event displays. The image at the top center shows the SciBath coordinate system. The plot on the lower right shows the hit time distribution within the event. Basic event and analysis summary information is included at the top right. 


\subsubsection{Fiducial volume cuts}

Fiducial volume cuts were used in each of the calculations discussed in this thesis. A fiducial volume cut requires that an event occur in an interior volume of the detector. For the muon flux analysis of Sec. 7.2 these cuts were used to avoid inaccurate reconstructions due to edge effects of the detector. These edge effects could be caused by reflections of light off of the aluminum walls of the detector cube or variations in WLS fiber density at the edge of the fiber grid. For the neutron capture detection analysis these cuts were additionally used to reduce the background event rate, which was higher near the edge of the detector where low energy particles tend to interact. The specific fiducial cuts used can be found in Table. 6.1. where two methods are noted: the box method and the sphere method.

\section{Box method}

The box fiducial method simply requires the average position of an event to be within a cube of adjustable size centered in the detector. This requirement was only used for neutron capture events where the fiducial cube was $31.7 \mathrm{~cm}$ on a side. Comparing to the full detector size, this is a $50 \%$ volume cut.

\section{Sphere method}

The sphere fiducial cut was used for muon events only. It required a muon track, reconstructed using the eigenvalue analysis of Sec. 6.4.3, to pass through a sphere of variable radius centered in the detector. Note that the average position of the energy response may not be within this sphere. For the muon flux measurement a sphere of diameter $31.7 \mathrm{~cm}$ was used, making the fiducial sphere volume $26 \%$ of the detector cube volume.

\subsubsection{Eigenvalue analysis for SciBath topology}

Given a set of points, $\alpha$, with associated masses, $m_{\alpha}$, one can calculate the directions of their

principle axes and the corresponding principle moments of inertia. This is done by building 
the inertia tensor, $T$, and solving the characteristic equation

$$
\operatorname{det}(T-\lambda I)=0
$$

where $I$ is the unit matrix and the roots, $\lambda$, are the principle moments of inertia. Vectors $\mathbf{v}_{\mathbf{n}}$ are then the principle axes of inertia if they satisfy

$$
T \mathbf{v}_{\mathbf{n}}=\lambda_{n} \mathbf{v}_{\mathbf{n}}
$$

The inertia tensor in three dimensions is

$$
\left(\begin{array}{ccc}
\overline{y^{2}}+\overline{z^{2}} & -\overline{x y} & -\overline{x z} \\
-\overline{x y} & \overline{x^{2}}+\overline{z^{2}} & -\overline{y z} \\
-\overline{x z} & -\overline{y z} & \overline{x^{2}}+\overline{y^{2}}
\end{array}\right)
$$

where

$$
\overline{x_{i} x_{j}}=\sum_{\alpha} m_{\alpha} x_{i, \alpha} x_{j, \alpha} \quad \text { and } \quad \overline{x_{i}^{2}}=\overline{x_{i} x_{i}}
$$

The SciBath eigenvalue analysis was done similarly, but with two differences. First, the SciBath data consists of light responses distributed across fibers instead of masses at points. Second, the energy response was divided out so that the analysis was independent of the event energy. The resulting tensor was

$$
\left(\begin{array}{ccc}
\overline{y^{2}}+\overline{z^{2}} & -\overline{x y} & -\overline{x z} \\
-\overline{x y} & \overline{x^{2}}+\overline{z^{2}} & -\overline{y z} \\
-\overline{x z} & -\overline{y z} & \overline{x^{2}}+\overline{y^{2}}
\end{array}\right)
$$

where

$$
\overline{x_{i} x_{j}}=\frac{\sum_{\alpha}\left(P E_{\alpha}\right)^{2} x_{i, \alpha} x_{j, \alpha}}{\sum_{\alpha}\left(P E_{\alpha}\right)^{2}} \text { and } \overline{x_{i}^{2}}=\overline{x_{i} x_{i}}
$$


Here $\alpha$ represents the set of fibers and $P E_{\alpha}$ refers to the photoelectron response of the photomultiplier tube channel that reads out fiber $\alpha$.

This analysis provides three eigenvectors and three corresponding eigenvalues that are analogous to principle axes of rotation and their corresponding moments of inertia. The energy deposit from a minimum ionizing muon track is cigar-shaped, similar to the ellipsoid in Fig. 6.11. The eigenvector corresponding to the smallest eigenvalue, analogous to the $c$-axis, is a reconstruction of the track direction. This reconstruction was used to draw the track directions in Fig. 6.10. The largest eigenvalue, analogous to the moment of inertia about the $a$-axis, is proportional to the track length and was used to separate muons and neutrons for energy deposits between 250 and 550 PEs, typical minimum ionizing muon energies.

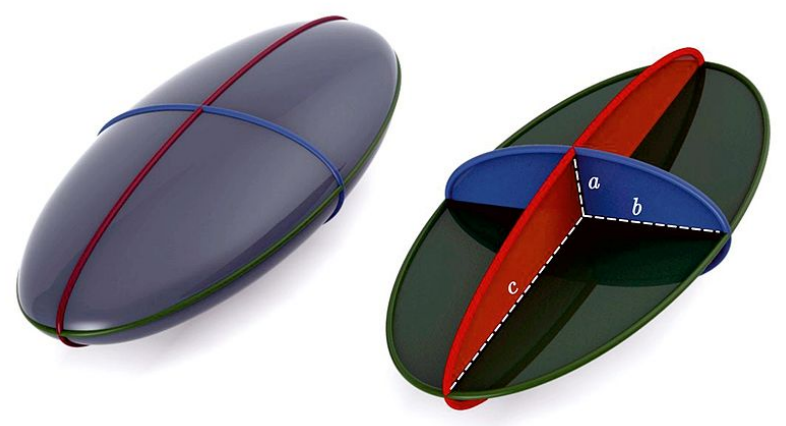

Figure 6.11: This ellipsoid is similar in shape to the energy deposition of a minimum ionizing particle in SciBath.

Figure 6.12 makes this separation apparent with data from the MI-12 run, described in Sec. 5.4. Figure 6.12a shows the largest eigenvalue versus photoelectron response for an event sample that is mostly composed of muons, while Fig. 6.12b shows the same for a sample that is mostly neutrons. 


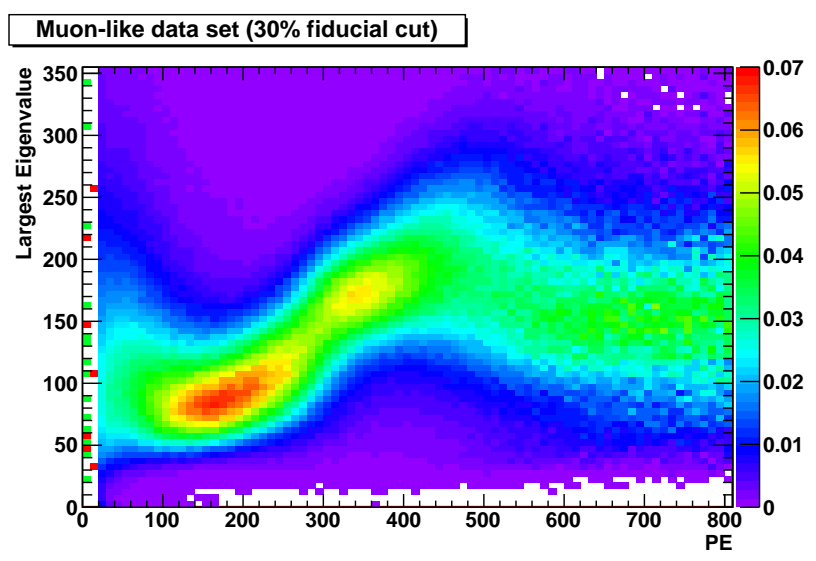

(a) Muon-like; beam uncorrelated.

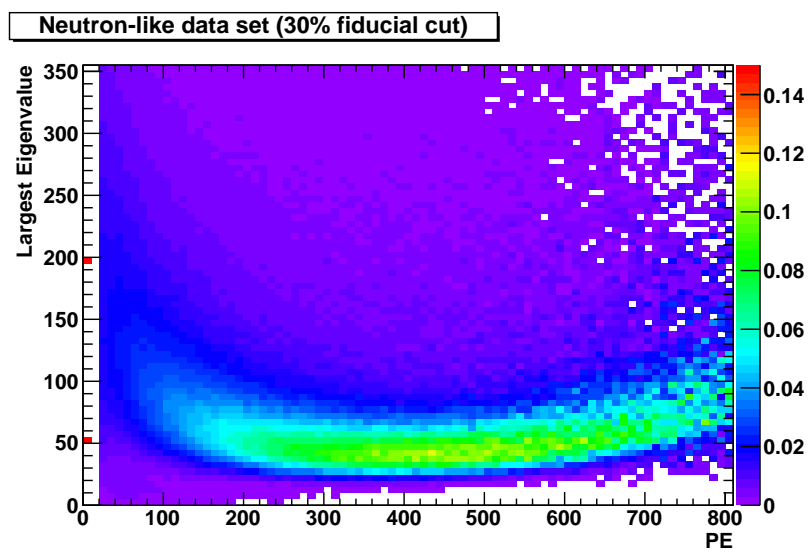

(b) Neutron-like; beam correlated.

Figure 6.12: Largest eigenvalue versus photoelectron response for beam uncorrelated (correlated) MI-12 events, which are primarily muons (neutrons) between 250 and 550 photoelectrons. These plots are count normalized in photoelectron response bins to clearly show the structure at higher photoelectron responses, which contain fewer events.

\subsection{Analysis cut summary}

Table 6.1 outlines the criteria used for event selection in this analysis. Timing cuts in reference to NuMI beam pulses are not included as all measurements of this analysis had the same beam timing cuts: greater than 2 ms after a beam trigger and therefore greater than 1.776(0.212) ms after(before) any beam pulse.

\begin{tabular}{lccc} 
Event type & Neutron scatter & Neutron capture & Muon \\
\hline \hline Hit multiplicity minimum & 6 & 6 & 6 \\
3D energy response & required & required & required \\
Hit time average (ns) & $(0, \infty)$ & $(0,40)$ & $(0, \infty)$ \\
Hit time sigma (ns) & $(0,100)$ & $(0,40)$ & $(0,100)$ \\
Fiducial method & $\mathrm{n} / \mathrm{a}$ & box & sphere \\
Fiducial volume & $100 \%$ & $50 \%$ & $26 \%$ \\
Largest eigenvalue $(\mathrm{PE} \geq 250)$ & $(0,115)$ & no cut & $(115, \infty)$ \\
Energy $(\mathrm{PE})$ & $(35, \infty)$ & $(12.5,35)$ & $(250, \infty)$ \\
\hline
\end{tabular}

Table 6.1: Event identification cuts summary. Since event timing cuts are only relevant for neutron capture events, they have not been included, see Sec. 6.2.1. 


\subsection{Background subtraction}

The neutron flux analysis required detection of a neutron scatter and the capture of the same neutron in coincidence. Similarly, the neutron production in scintillator analysis required detection of a muon track and the capture of a neutron produced by that muon. These signals were contaminated by coincidence event pairs where either or both of the primary and/or secondary events were not actually caused by the desired correlated interactions. Even if the primary and secondary events were neutron scatter and capture, for example, they may not have been from the same neutron. In this section I first describe how the subtraction to get the true event count was done. I then describe the extension of this subtraction to time and energy distributions.

\subsubsection{Uncorrelated neutron capture candidate rate}

The data set for this analysis contains many more candidate neutron capture events than candidate neutron scatter events. This is primarily a result of the difference in the energy cuts, see Table 6.1, and the higher event rates at lower energies. As a result, all neutron scatter candidate events were stored for the analysis, but only neutron capture candidates that were correlated to a neutron scatter candidate. To account for background events we were thus required to calculate the average neutron capture candidate rate for the run.

This was done by building a spectrum of the time between neutron scatter and neutron capture candidates $(\Delta \mathrm{T})$ and letting the time range far exceed the expected lifetime of neutron capture in scintillator. Figure 6.13 shows the fit of this spectrum with a decaying

exponential, which resulted in a background level. Dividing this by the time bin width and the number of correlations used, I found an overall rate of $22.98 \mathrm{~Hz}$. 


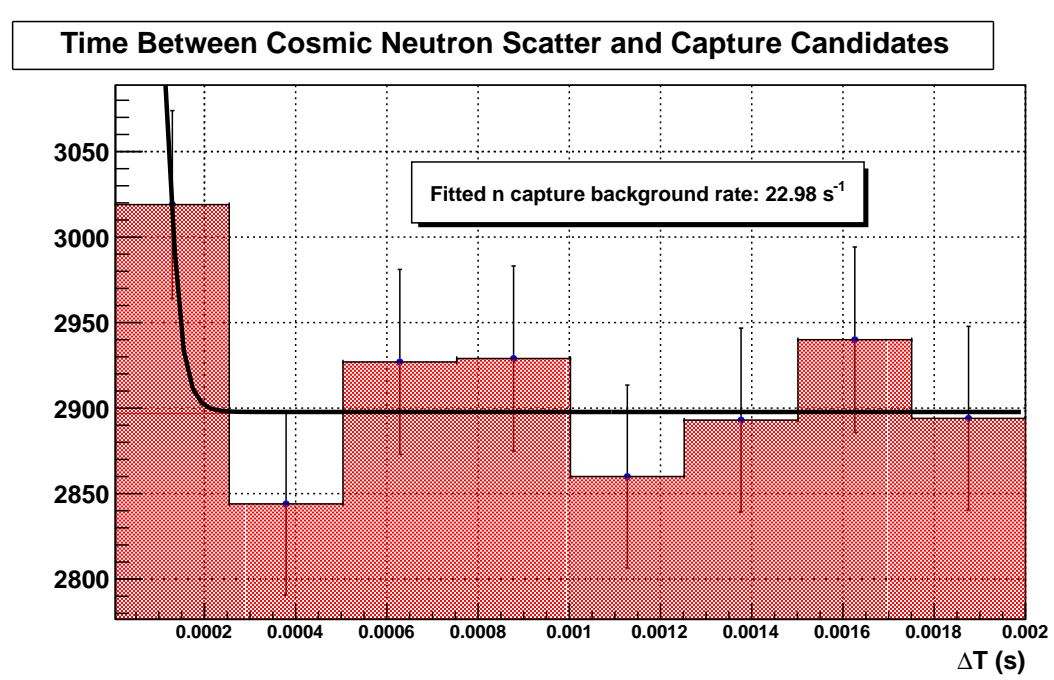

Figure 6.13: Fit of the time difference between neutron scatter and capture candidate events. The time range is much larger than the $163 \mu$ s average time difference to allow an accurate fit of the accidental background rate for neutron capture candidates.

\subsubsection{Incident neutron event counting}

A counted neutron primary must be correlated in time with a secondary neutron event, which may be the capture of the neutron responsible for the primary event or may be an uncorrelated secondary event. The number of neutron primary events that are correlated to their capture can be expressed as

$$
P_{s, c}=P_{s, T}-P_{s, u},
$$

where

$$
\begin{aligned}
& P_{s, c} \equiv \text { number of primaries with correlated secondaries, } \\
& P_{s, T} \equiv \text { total number of primaries with secondaries, and } \\
& P_{s, u} \equiv \text { number of primaries with uncorrelated secondaries. }
\end{aligned}
$$

The number of primaries with uncorrelated secondaries, $P_{s, u}$, can be expressed as the product of the number of primaries available for accidental correlation and the the probability 
that an uncorrelated secondary event would occur in the neutron capture time window. Naively, one would think this probability is simply the time width of the neutron capture window, $\Delta \mathrm{T}$, multiplied by the uncorrelated secondary rate of Sec. 6.6.1, but this assumes that uncorrelated secondaries are evenly distributed in time. In reality, of course, such uncorrelated events are randomly distributed. Therefore some neutron capture windows will contain multiple uncorrelated events and more such windows will contain zero events than the naive guess would suggest. To quantify this effect, secondary candidate events were counted in random windows of width $\Delta \mathrm{T}$ throughout the data run. The ratio of the number of windows containing at least one event to the total number of events counted gives the correction factor for this effect, $C$. For the analysis cuts listed in Table 6.1 the factor $C$ was 0.9974 , which was close enough to one to be insignificant. With wider cuts on neutron capture candidates, however, $C$ would shrink and becomes more significant.

Equation 6.13 can now be written

$$
P_{s, c}=P_{s, T}-\left(P_{T}-P_{s, c}\right) \cdot \Delta T \cdot R_{s, u} \cdot C
$$

where

$$
\begin{aligned}
& P_{T} \equiv \text { total number of primaries with or without secondaries, } \\
& \Delta T \equiv \text { width of the secondary event time window, } \\
& R_{s, u} \equiv \text { uncorrelated secondary rate, and } \\
& C \equiv \text { correction factor for the random time distribution of uncorrelated secondaries. }
\end{aligned}
$$

The target neutron event count, $P_{s, c}$, is present on both sides of Eqn. 6.14 because the number of primaries available for accidental correlation should not include those primaries 
with true correlations. Solving for $P_{s, c}$ gives

$$
P_{s, c}=\frac{P_{s, T}-P_{T} \cdot \Delta T \cdot R_{s, u} \cdot C}{1-\Delta T \cdot R_{s, u} \cdot C} .
$$

\subsubsection{Secondary event counting}

The secondary event count subtraction proceeds similarly to the primary event subtraction above. The number of correlated secondary events can be expressed as

$$
S_{p, c}=S_{p, T}-S_{p, u},
$$

where

$$
\begin{aligned}
& S_{p, c} \equiv \text { number of secondaries following correlated primaries, } \\
& S_{p, T} \equiv \text { total number of secondaries following primaries, and } \\
& S_{p, u} \equiv \text { number of secondaries following uncorrelated primaries. }
\end{aligned}
$$

The number of secondaries following uncorrelated primaries, $S_{p, u}$, can be expressed as the product of the time available for accidental secondary events and the uncorrelated secondary rate of Sec. 6.6.1. $R_{s, u}$. The available time for accidental secondaries is simply the total number of primaries available for accidental correlation multiplied by the time width of the neutron capture window, $\Delta T$. Thus, Eqn. 6.16 can be rewritten as

$$
S_{p, c}=S_{p, T}-\left(P_{T}-P_{s, c}\right) \cdot \Delta T \cdot R_{s, u},
$$

where the number of correlated primaries, $P_{s, c}$, is again subtracted from the total number of primaries, $P_{T}$, to give the total number of primaries available for accidental correlation.

A correction factor like $C$ in the primary subtraction, Sec. 6.6.2, is not required in this subtraction because all correlated secondaries are being counted, regardless of whether or not 
multiple occur in the same neutron capture window. This difference allows the secondary event count to be higher than the primary event count, which could result from neutron inelastic scattering with carbon, which can release additional neutrons. In practice, the difference between primary and secondary event counts was less than $2 \%$. In principle, a similar correction factor could be included to account for multiple primaries occurring in time such that they are collectively only allowed to open a single neutron capture window, but in practice the event rate for primary events was low enough that this correction was unnecessary.

\subsubsection{Extension to energy and time distributions}

The background subtraction of energy and time distributions for neutron events was performed using the methodology of Sec. 6.6.2 and Sec. 6.6.3 except that I multiplied the minuend and subtrahend of each subtraction with the appropriate distributions, normalized to one. The appropriate distribution for the minuend is generally obvious: the time or energy distribution for primaries correlated to secondaries or secondaries correlated to primaries. The subtrahend energy distributions are then the compliment of the minuend distributions: primaries with no correlated secondaries or secondaries with no correlated primaries. For simplicity in the secondary energy subtraction, I used instead the energy distribution of all secondary candidates for the secondary subtrahend distribution since this distribution is dominated by secondaries without correlated primaries and is easier to generate. Since uncorrelated secondary candidate events do not have a time measurement after a primary, the data does not directly provide the subtrahend distribution for the primary-to-secondary time difference subtraction. Instead I built this as a flat distribution at the level expected by the uncorrelated secondary rate described in Sec. 6.6.1. 


\subsection{Monte Carlo simulation}

Every raw scientific measurement must be compared to an expectation, or model, for interpretation. This model should incorporate all possible factors that will effect the raw measurement, which include the physical phenomena being tested as well as natural background influences and the efficiency and systematic effects of hardware, analysis software, and analysis algorithms. Modern scientific measurements are extremely complex with a large number of possible influences, beyond the estimation capabilities of analytic calculations. Instead these measurements depend upon computationally intensive simulations that attempt to numerically account for all significant influences on a measurement.

This analysis utilized the Geant4 [61, 62] and MCNP [63] Monte Carlo simulation toolkits. Geant4 is a toolkit based on object oriented programming in $\mathrm{C}++$ that was developed by the European Organization for Nuclear Research (CERN) and is maintained by the Geant4 Collaboration. The toolkit was designed to simulate the passage of particles through matter and it includes features to handle detector response, run management, geometry, tracking, visualization, and user interface. Geant4 has been applied to the fields of nuclear and high energy physics experiments as well as accelerator, medical, and space physics studies. The SciBath Geant4 simulation was utilized for the detailed and prompt response of the detector to neutrons, muons, and photons.

The Monte Carlo N-Particle (MCNP) package is a coupled neutron, photon, and electron radiation transport code that has been used extensively in many fields such as high-energy physics, nuclear reactors, oil well logging, accelerator design, and medical physics. It was developed and is maintained by the Los Alamos National Laboratory (LANL), though it is distributed by the Radiation Safety Information Computational Center within the United States and by the Nuclear Energy Agency internationally. MCNP is generally considered the superior low-energy neutron simulation package and was used to perform these simulations in SciBath. Additionally, MCNP simulations were used to validate the Geant4 simulation results. 


\subsubsection{Geant4 Monte Carlo simulation}

The Geant4 toolkit [61, 62] was used to model the geometry of the SciBath detector, track particles through the volumes, propagate the resulting visible photons, and simulate the photodetector response to those visible photons. A list of physics packages used can be found in Appendix A.

The simulated geometry consisted of the aluminum cube containing the active scintillator and WLS fiber grid. In addition, the major pieces of the support frame, important for possible particle interactions, were input. Geant4 code was then used to generate and to transport various particles with the desired initial kinematic and position distributions. The scintillator was modeled as mineral oil $\left(\mathrm{CH}_{2}\right)$ of density $0.86 \mathrm{~g} / \mathrm{cm}^{3}$ and index of refraction $n=1.476$. Charged particles produced visible photons isotropically in proportion to their energy loss at each tracking step. The number of photons produced was controlled with an input parameter set to 1500 photons per $\mathrm{MeV}$ of energy loss. This was tuned to match muon data, see Sec. 6.3.1, and was validated by comparisons with neutron data. The reduction in this light output compared to the 7000 photons per $\mathrm{MeV}$ given in Sec. 4.3 may have been caused by attenuation in fibers, fiber coupling, or possible oxygen poisoning of the scintillator, and is currently under investigation. Quenching of light for particles of high $d E / d x$ with a Birks' Law [58] response, see Sec. 6.3.2, was included in this simulation. The WLS fiber volumes were modeled as separate volumes of the same liquid scintillator with positions matching the actual detector.

The photons produced in the scintillator were tracked by Geant 4 until they were absorbed in the scintillator due to a finite attenuation length of $10 \mathrm{~m}$, absorbed on the $\mathrm{Al}$ walls which did not reflect light, or absorbed by a WLS fiber. The details of optical photon transport in the WLS fibers were not modeled. If a visible photon intersected the fiber volume it was absorbed immediately and considered detected with a probability of $2.5 \% \times 20 \%$, the product

of the optical photon capture probability within the WLS fiber with the photomultiplier tube (PMT) detection efficiency. A detected photon was called a "photoelectron" (PE) to 
reflect the detection process within the PMT even though it was not explicitly simulated. For muon simulations, the total PE response was subjected to a Gaussian smearing with width $=0.59 \mathrm{PE}$, calculated from calibration runs, and a detection threshold of $1 / 3 \mathrm{PE}$ to account for the ADC threshold. This smearing was not implemented at the time of the neutron simulations. A simulated muon track is shown in Fig. 6.14.

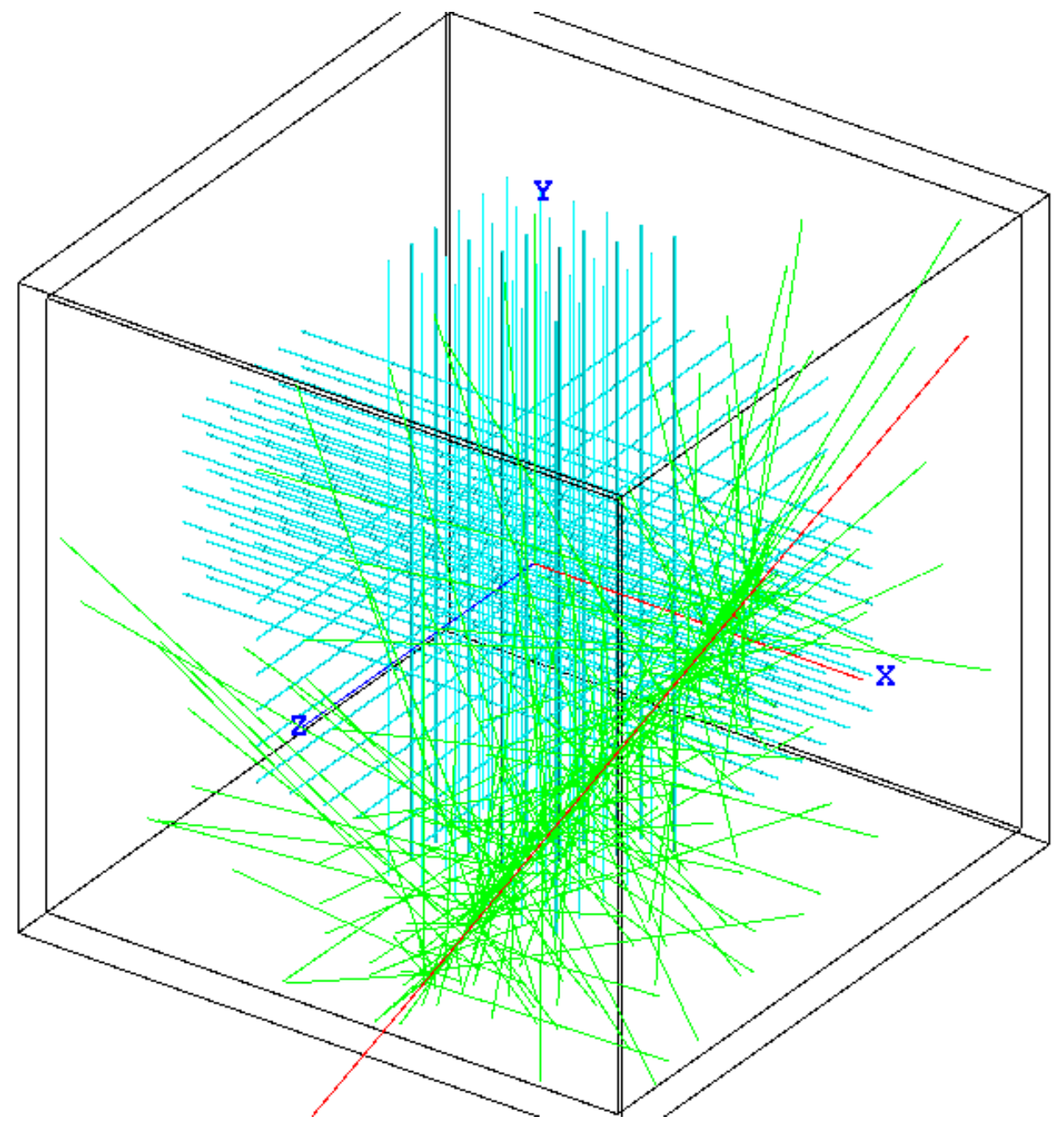

Figure 6.14: Geant4 simulation of a $19.4 \mathrm{GeV}$ muon in the SciBath detector. The muon track is red and the scintillation photon tracks are green. The number of scintillation photons was reduced by a factor of 3 for this event to enable details to be seen.

The data from each simulated event consisted of a number of detected photons for each fiber along with the "truth" information about the incident particle(s). This information was then analyzed with the same methods as those used on real data and the response of 
the detector to known incident particles was calculated.

\subsubsection{MCNP Monte Carlo simulation}

Various flavors of the MCNP simulation package [63] were used to validate the Geant4 simulations. In these simulations, a simplified model of the detector was used, and the complete detector response was not considered. Despite this, we were able to compare to Geant4 by calculating neutron capture efficiencies and neutron interaction cross sections. In these simulations we primarily used MCNP5 release version 1.51 664. MCNP5 has a wellknown non-analog production of neutron-capture gamma rays, and the production of these photons (e.g. 2.2 MeV photons from the $\mathrm{np} \rightarrow \mathrm{d} \gamma$ reaction) was simulated with MCNPPoliMi [65]. PoliMi is a modified version of the older MCNP4C that corrects this problem and ensures that photon production is fully correlated with each neutron interaction. A template for our MCNP simulations can be found in Appendix B.

The MCNP simulation assumed a box of hydrocarbon in a 2:1 ratio of $\mathrm{H}: \mathrm{C}$ with the same dimensions as the SciBath detector. This box was surrounded by $1.27 \mathrm{~cm}$ of natural aluminum. The scintillating fibers were not included in this simulation, nor was other surrounding equipment. The latest room temperature ENDF evaluations for hydrogen and carbon [66] were used depending on the maximum neutron energy (tables with a maximum energy of $20 \mathrm{MeV}$ or $150 \mathrm{MeV}$ were available). Because the neutron capture cross section varies with the neutron velocity $v$ by the well-known $1 / v$ law, thermal effects in the mineral oil can strongly effect the neutron capture efficiency. These effects are encapsulated by MCNP with the $S(\alpha, \beta)$ model, and we used the table for hydrogen in water as a substitute for hydrogen in mineral oil.

MCNP was used to calculate a number of interaction and capture efficiencies for SciBath. A uniform background of neutrons was simulated by having neutrons emitted in a cosine distribution, uniformly across the surface of a "bounding" sphere. In this case, a cosine distribution leads to neutron fluxes with no preferred direction. This was used to calculate the 
effective cross-sectional area of the detector for neutron interaction as a function of the incident neutron energy. These simulations were used to calculate the neutron capture efficiency and positional dependence for the uniform backgrounds. PoliMi was used to simultaneously calculate the efficiency of neutron capture, see Sec. 6.7.4. PoliMi has an upper energy limit of $20 \mathrm{MeV}$, and above that a separate photon transport calculation was convolved with the neutron capture distribution. Both methods were in agreement below $20 \mathrm{MeV}$. Finally, cosmic ray muons will create neutrons within the bulk of the detector. The neutron capture efficiency was calculated for neutrons "born" within the bulk of the detector as a function of their energy, see Sec. 6.7.5.

\subsubsection{Simulation of neutron capture tagging}

As described in Sec. 2.4.4 this analysis identified neutrons by requiring two events in coincidence: a neutron scatter and a neutron capture. To demonstrate the effects of the coincidence requirement, neutron interactions were simulated in Geant4 as described in Sec. 6.7.1. For the sample in Fig. 6.15a the neutrons were not required to capture inside the detector and the reconstructed energy is often much lower than the incident neutron energy. This is because many neutrons will scatter one or more times and then leave the detector without depositing all of their energy. For comparison, Fig. 6.15b shows the same energy reconstruction versus incident neutron energy, but only includes neutrons that capture inside the detector. This gives a much improved, more "diagonal," energy reconstruction. Of course, a perfect reconstruction would be a narrow line near the top edge of the distribution. Even in simulation the reconstruction is smeared due to the quenching effects described in Sec. 6.3.2, losses to neutron-carbon inelastic scattering, and statistical variation in detector response.

\subsubsection{Externally generated neutron detection efficiency}

For a neutron that enters SciBath to be detected with this analysis, three requirements must be met: 


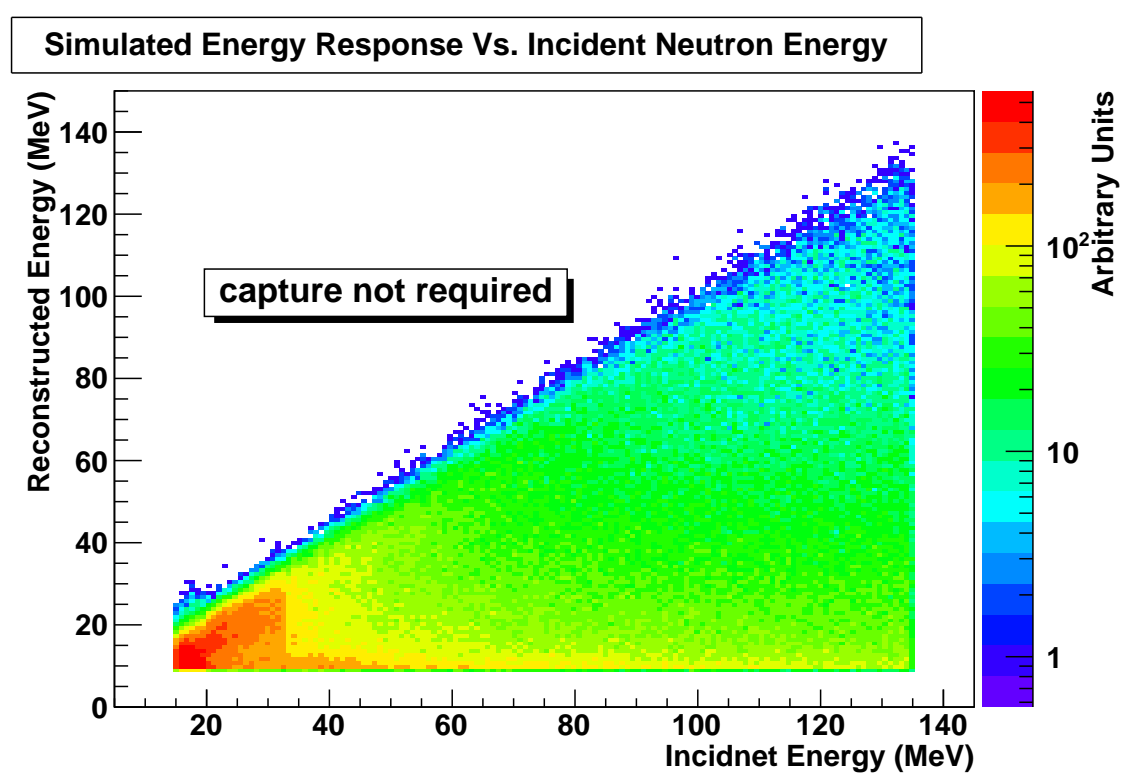

(a) Neutron capture not required.

\section{Simulated Energy Response Vs. Incident Neutron Energy}

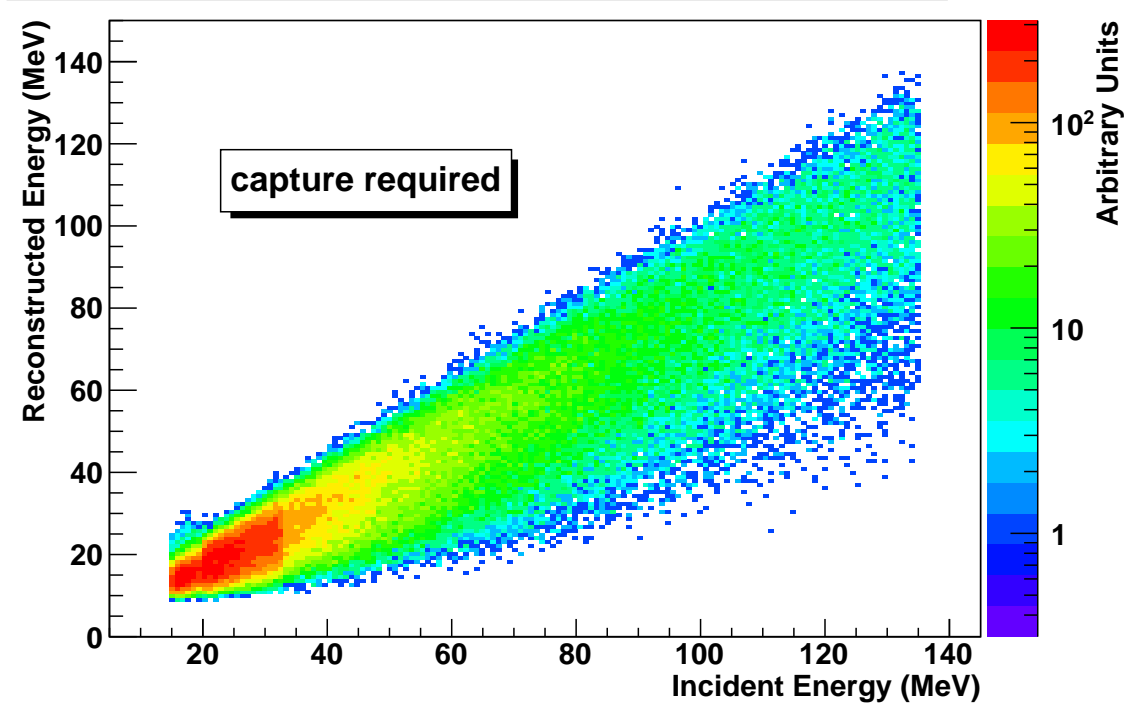

(b) Neutron capture required.

Figure 6.15: Geant4 simulated photoelectron response for neutron scattering events versus incident neutron energy. Requiring neutron capture ensures the photoelectron response is much more correlated to the incident neutron energy. 
1. The neutron must scatter off of one or more hydrogen nuclei such that the recoil proton(s) generate a detector response of at least 35 photoelectrons. Based on the cuts in Table 6.1 and the quenching correction of Sec. 6.3.2, this would be satisfied by a single recoil proton of energy greater than $16 \mathrm{MeV}$.

2. The neutron must thermalize and capture on a proton inside the detector releasing a $2.2 \mathrm{MeV}$ photon.

3. The capture photon must Compton scatter inside the detector such that the recoiling electron generates a detector response of at least 12.5 photoelectrons. Based on the cuts in Table 6.1 and the energy calibration of Sec. 6.3.1, this would be satisfied by a single recoil electron of reconstructed energy greater than $2.5 \mathrm{MeV}$.

Table 6.2 summarizes the contributions to the external neutron detection efficiency.

\begin{tabular}{cc} 
Process & Efficiency \\
\hline \hline neutron scatter detection & 0.92 \\
neutron capture & 0.16 \\
$2.2 \mathrm{MeV} \gamma$ detection & 0.062 \\
$\Delta \mathrm{T}$ cuts & 0.68 \\
neutron enter detector & 0.63 \\
\hline total & 0.0039 \\
\hline
\end{tabular}

Table 6.2: Summary of the external neutron detection efficiency. The "neutron enter detector" efficiency is specific to the simulation used and is for an assumed SciBath cross section of $\pi(40 \mathrm{~cm})^{2}$, larger than the detector cube.

To address requirement one, the SciBath efficiency to detect neutron scattering interactions that were correlated to detected neutron capture events for neutrons between 16 to $156 \mathrm{MeV}$ was assessed. Geant4 simulated neutrons less than $300 \mathrm{MeV}$ were generated on the surface of a sphere surrounding the detector with uniform angular distribution inward. These neutrons were required to deposit at least $99 \%$ of their energy inside the detector to ensure the neutron could have captured. Analysis cuts for neutron scatters were then applied, resulting in an efficiency of 0.92. To the level that Geant4 handles inelastic neutron 
interactions, this analysis suggests that at least $92 \%$ of neutrons above $16 \mathrm{MeV}$ undergo detectable neutron-proton elastic scattering interactions. Note that the energy spectrum used in this simulation was flat and thus not representative of the true neutron energy spectrum. This suggests a correction as the neutron scatter detection efficiency is energy dependent, but we believe the correction is relatively minor compared to the other uncertainties of this measurement.

The efficiency for neutrons to capture was evaluated using an MCNP5 simulation. Neutrons were generated with a uniform $2 \pi$ angular distribution on the inner surface of a sphere containing an aluminum cube of liquid scintillator matching the dimensions of SciBath. These neutrons were propagated and the number that entered the detector, the number of those that captured in the detector, and the locations of those captures were recorded. This method gave the efficiency for requirement 2 above and resulted in the distribution in Fig. 6.16. To find an overall effective neutron capture efficiency a weighted average of this distribution was calculated with our measured neutron energy spectrum, Fig. 7.8, The resulting efficiency was 0.16 .

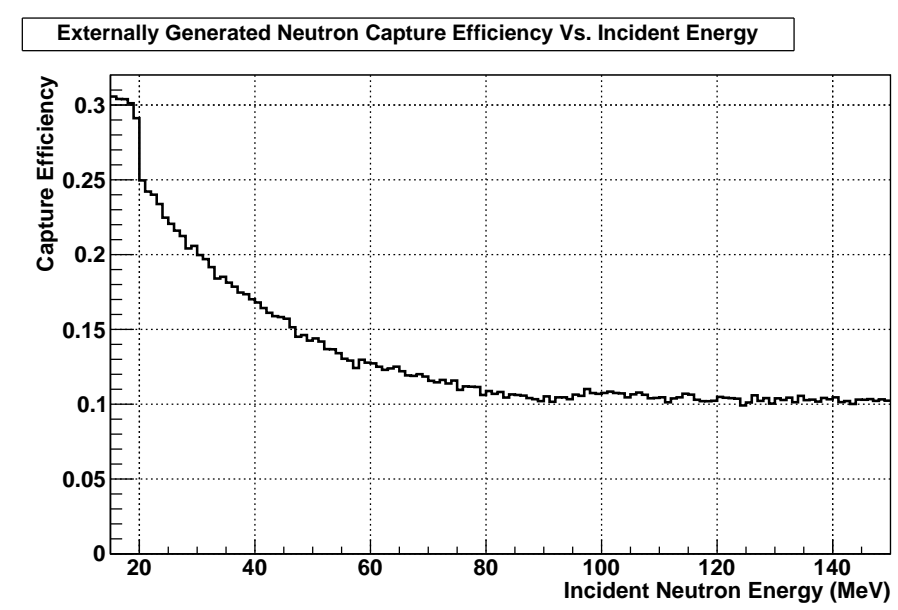

Figure 6.16: External neutron capture efficiency for neutrons entering SciBath as determined by simulation of neutrons generated uniformly on a sphere surrounding the detector. Detection of the $2.2 \mathrm{MeV}$ capture photon is not included in this efficiency. 
To assess the SciBath efficiency to detect neutron capture events, 2.2 MeV photons were generated uniformly throughout the majority of the scintillator volume in a Geant4 simulation. The previously described MCNP5 neutron simulation indicated that fewer neutrons capture near the edges of the detector, so photons were only generated in the inner $70 \%$ of the detector volume. The fraction of these that deposited energy inside the detector was recorded as well as the amount of energy deposited and generation location of the photon. The fraction of these that created events that passed the neutron capture candidate cuts was 0.062 and is the efficiency for the requirement 3 above.

For the neutron flux calculation two more efficiencies need to be included. First, since neutron primaries and secondaries were simulated separately, the efficiency to pass the $\Delta \mathrm{T}$ cut (the time between a neutron primary and its capture secondary) has not been accounted for. This efficiency was assessed by fitting beam correlated capture candidates with a decaying exponential, see Fig. 7.13, and then integrating that exponential over the $\Delta \mathrm{T}$ cut range. For cosmic neutron cuts, this gave an efficiency of 0.68 . Second, only $63 \%$ of the neutrons generated in the described MCNP5 simulation actually entered the detector, thus when using this simulation an extra 0.63 efficiency needs to be included. This added efficiency can be thought of as an adjustment to the neutron cross section of SciBath from the simulated neutron generation sphere cross section.

The product of these five efficiencies gives an overall external neutron detection efficiency of 0.0039, as summarized in Table 6.2.

\subsubsection{Internally generated neutron detection efficiency}

Section 6.7 .4 describes the efficiency for this analysis to detect fast neutrons that enter SciBath. A measurement was also made, however, of the neutron production rate by cosmic muons in the liquid scintillator itself, see Sec. 7.4. For a neutron produced by a (detected) muon inside SciBath to be detected with this analysis, only two requirements must be met:

1. The neutron must thermalize and capture on a proton inside the detector releasing a 


\subsection{MeV photon.}

2. The capture photon must Compton scatter inside the detector such that the recoiling electron generates a detector response of at least 12.5 photoelectrons. Based on the cuts in Table 6.1 and the energy calibration of Sec. 6.3.1, this would be satisfied by a single recoil electron of reconstructed energy greater than $2.5 \mathrm{MeV}$.

This differs from the analysis for externally produced neutrons in three ways. First, no neutron scattering event is required, though one may be present; and if so, it would be considered part of the muon event. Second, this analysis imposes no requirements on neutron energy; all that is required is that the neutron captures. This is an important consideration because as Fig. 3.4 shows, most neutrons spallated by muons are low energy. Third, the position at which the neutron thermalizes in the detector is different between externally and internally produced neutrons, thus affecting the likelihood that the neutron will escape the detector before capturing. Table 6.3 summarizes the contributions to the internal neutron detection efficiency.

\begin{tabular}{cc} 
Process & Efficiency \\
\hline \hline neutron capture & 0.42 \\
$2.2 \mathrm{MeV} \gamma$ detection & 0.062 \\
$\Delta \mathrm{T}$ cuts & 0.68 \\
\hline total & 0.018 \\
\hline
\end{tabular}

Table 6.3: Summary of the internal neutron detection efficiency.

The fraction of internally generated neutrons to capture was assessed with a MCNP simulation. Neutrons were generated uniformly inside an aluminum cube containing liquid scintillator matching the dimensions of the SciBath detector cube. These neutrons were between 0.1 and $150 \mathrm{MeV}$ and had a uniform $4 \pi$ angular distribution. The fraction of these

neutrons that captured as a function of initial energy is plotted in Fig. 6.17. A weighted average of this distribution was taken with the Da Silva et al. analytic form for neutron energy from spallation in wax [67] as the energy weighting. The resulting neutron capture efficiency was 0.42 . 
The 0.062 efficiency for detection of the neutron capture photon, Sec. 6.7.4, was again used for internal neutrons. Similarly, the 0.68 for the $\Delta \mathrm{T}$ cut also found in Sec. 6.7.4 was used since the same timing window was used. The product of these three efficiencies gives an overall internal neutron detection efficiency of 0.018 , as summarized in Table 6.3.

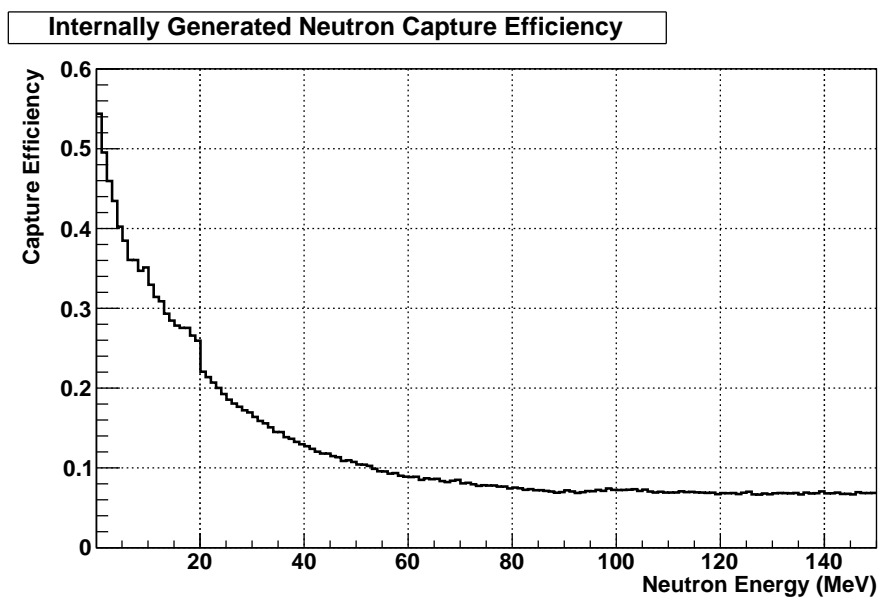

Figure 6.17: Neutron capture efficiency for SciBath for internally generated neutrons, as determined by a simulation of neutrons generated uniformly in the detector. Detection of the $2.2 \mathrm{MeV}$ capture photon is not included in this efficiency.

The internally generated neutron capture efficiency was also studied as a function of starting distance from the center of the detector. The result is plotted in Fig. 6.18. This position dependence was used to assess the contamination in the neutron flux measurement caused by muons that are tagged as neutron scattering events and also spallate neutrons that then capture inside the detector, thus meeting the external neutron identification criteria. This contamination assessment is described in Sec. 7.5.

\subsubsection{Muon detection efficiency}

The SciBath muon detection efficiency was assessed to allow the interpretation of our muon count as a muon flux measurement. The Geant4 simulation of SciBath described at the start of Sec. 6.7 was used to propagate muons generated uniformly on the inside of a $40 \mathrm{~cm}$ radius hemisphere centered on the detector with an angular distribution matching that given by 


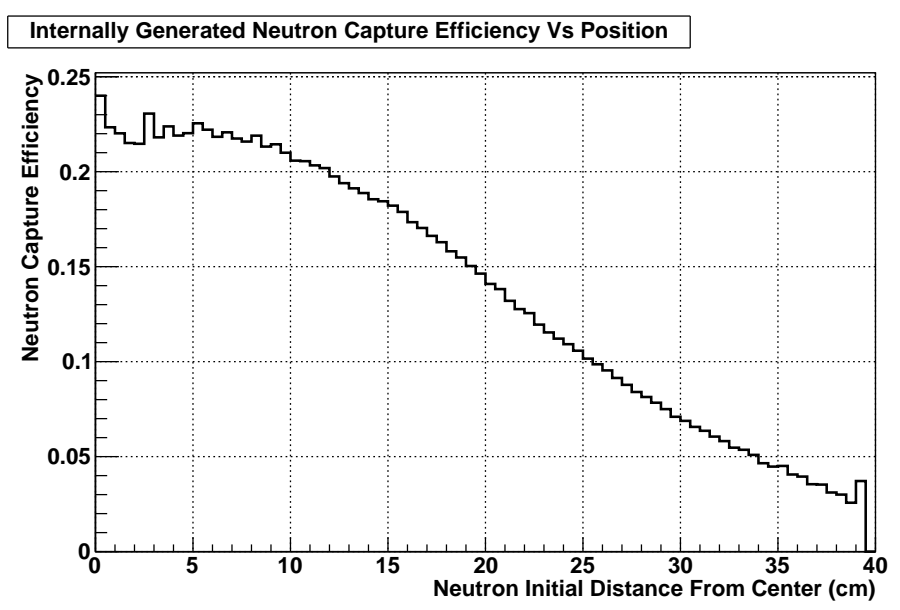

Figure 6.18: Neutron capture efficiency for SciBath for internally generated neutrons, as determined by a simulation of neutrons generated uniformly in the detector. The efficiency for detection of the $2.2 \mathrm{MeV}$ capture photon is not included in this efficiency.

Miyake [13]. The portion of these muons that were propagated into the detector had to pass the analysis cuts of Table 6.1. Of the 1797110 muons generated, 281768 of which passed through the detection sphere, 340329 passed the cuts and were considered detected. This simulation thus estimates the SciBath efficiency for detecting cosmogenic muons that pass through the detector to be 0.1894 , for the chosen cuts. Note that more events were counted after event reconstruction than were expected at generation. The efficiency for this analysis to detect muons through the detection sphere was 1.21. This shows a tendency in the track reconstruction algorithm to pull muon tracks toward the center of the detector and can be thought of as an increase in the fiducial volume used for muon identification.

\subsubsection{Muon track length}

The measurement of muon induced neutron spallation in scintillator, Sec.7.4, was normalized to a unit muon track length. Thus the average track length of muons in SciBath was needed. The same Geant4 cosmic muon simulation described in Sec. 6.7.6 was used to generate the track length distribution in Fig. 6.19a. This distribution is for muons that pass muon identification cuts, as opposed to Figs. 6.19b and 6.19c which show the distributions for 
simulated muons that fail these cuts but pass neutron identification cuts. Since the SciBath detector cube is $44 \mathrm{~cm}$ on each side, a muon traversing the detector at a right angle to a side of the cube has a path length of $44 \mathrm{~cm}$. Most cosmic muons are nearly vertical and therefore have a track length near $44 \mathrm{~cm}$. Muons of other angles can yield paths lengths between $0 \mathrm{~cm}$ and $44 \sqrt{2}=62 \mathrm{~cm}$ depending on the geometry. The average track length of the misidentified muons is required to assess the muon contamination in the neutron flux measurement, Sec. 7.5 .

\subsubsection{Muon average position}

The same Geant4 cosmic muon simulation described in Sec. 6.7.6 was analyzed using muon and neutron identification cuts, and the resulting average distance of energy response from the detector center is plotted in Fig. 6.20. Here the average distance of energy response was actually increased by $5 \%$ to account for the tendency of the topological analysis to reconstruct events closer to the center of the detector than the actual energy deposit. The $5 \%$ factor was selected by comparing simulated and reconstructed muon tracks by eye. These position distributions are important for determining the contamination in the neutron flux measurement by muons that are misidentified as neutrons due to their ability to spallate neutrons in the scintillator, see Sec. 7.5. 


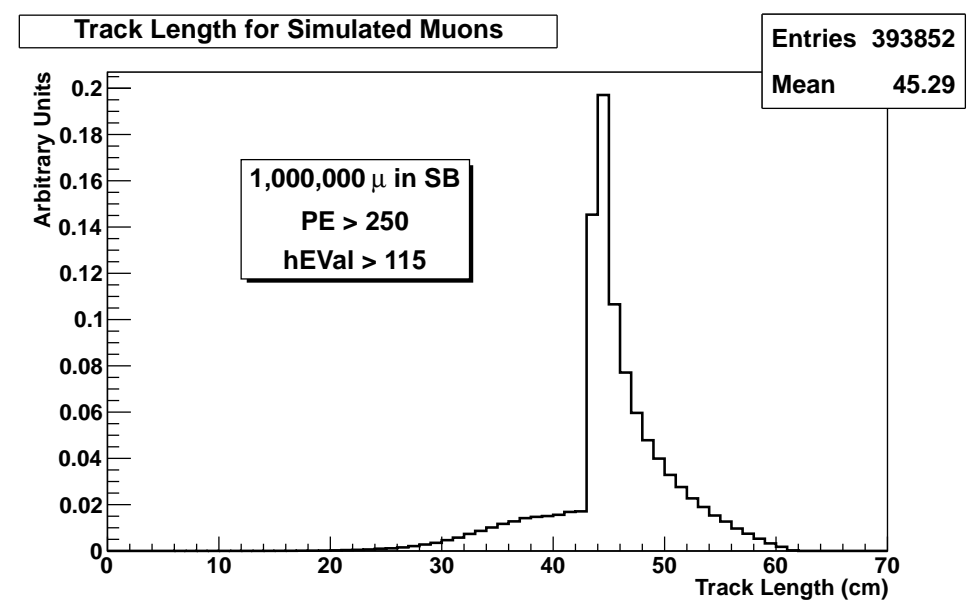

(a) Simulated muons that pass muon identification cuts.

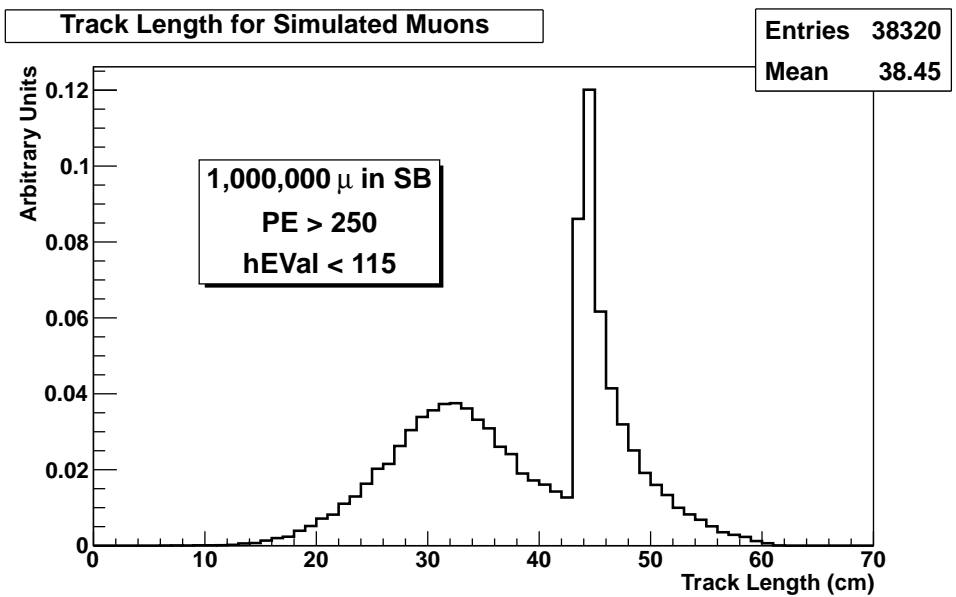

(b) Simulated muons that pass neutron identification cuts above 250 PEs.

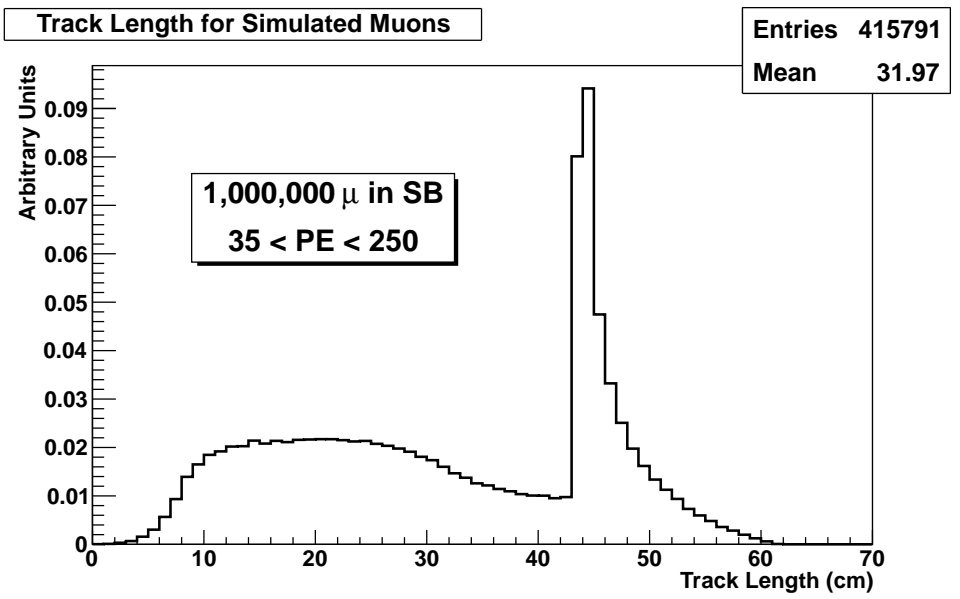

(c) Simulated muons that pass neutron identification cuts below 250 PEs.

Figure 6.19: Simulated cosmic muon track length distributions. 


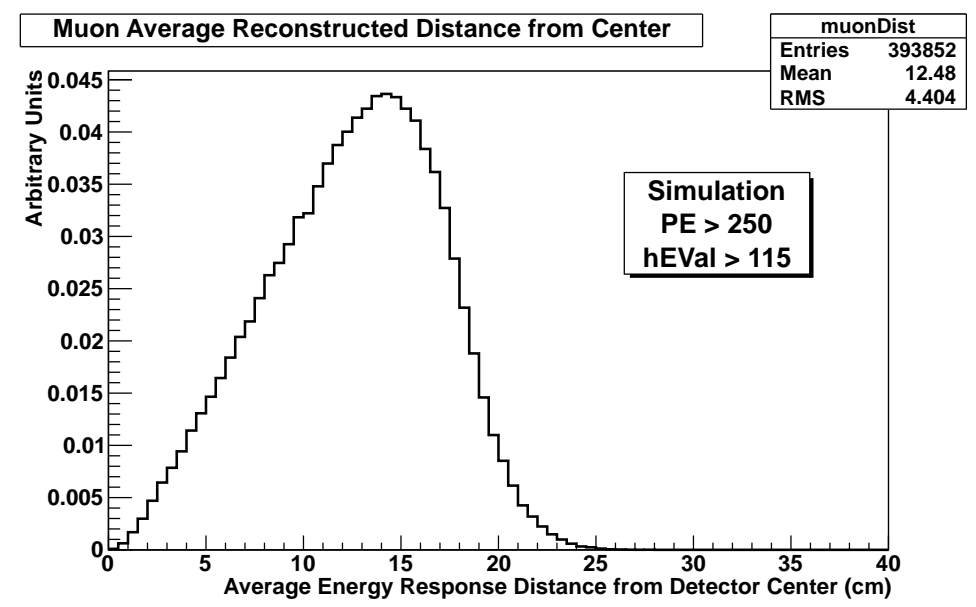

(a) Simulated muons that pass muon identification cuts.

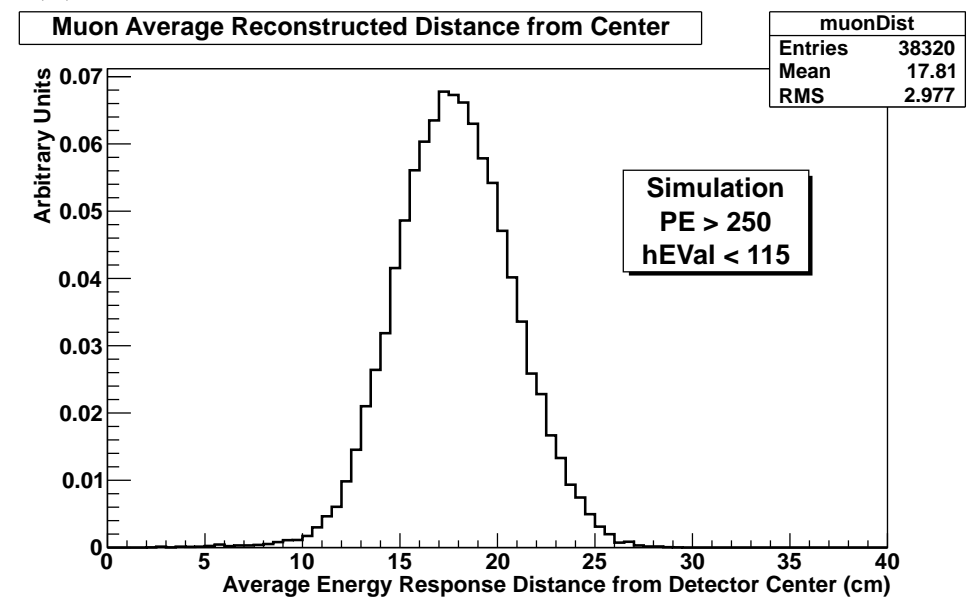

(b) Simulated muons that pass neutron identification cuts above 250 PEs.

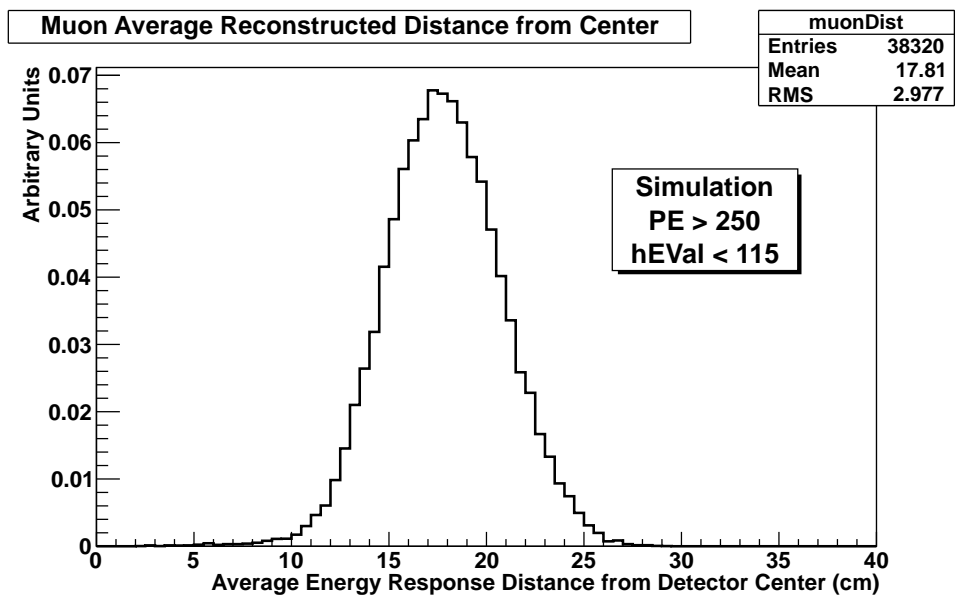

(c) Simulated muons that pass neutron identification cuts below 250 PEs.

Figure 6.20: Simulated muon reconstructed distance of average energy response from the detector center. 


\section{Chapter 7}

\section{Results}

The experiment and analysis described in this thesis culminate with a series of results on neutron and muon characteristics at $100 \mathrm{~m}$ underground. In this chapter I present these results starting in the first section with the analysis that gave rise to their associated uncertainties, which are relatively large for the neutron results. The following two sections present the muon flux and angular distribution measurements, both of which are in agreement with previous measurements and predictions. The muon flux was found to be $0.80 \pm 0.04 \mathrm{~m}^{-2} \mathrm{~s}^{-1}$. In section $7.4 \mathrm{I}$ present the muon-induced neutron spallation rate, measured at $(3.8 \pm 3.2) \times 10^{-4} \mathrm{n} / \mu\left(\mathrm{g} / \mathrm{cm}^{2}\right)^{-1}$. This result is high compared to other measurements and predictions, but agrees within error. In the fourth and fifth sections I present the neutron flux and energy spectrum results. The neutron flux was measured to be $(1.5 \pm 1.4) \times 10^{-2} \mathrm{~m}^{-2} \mathrm{~s}^{-1}$ and is high compared to the prediction by Mei and Hime [14], but agrees within error. In section $7.7 \mathrm{I}$ present the ratio of the neutron and muon fluxes as $(1.9 \pm 1.7) \times 10^{-2}$ neutrons per muon, which may be more easily applied to other locations than the neutron flux alone. In section 7.8 I describe a validation analysis based on NuMI beam correlated data. Finally, in Sec. 7.9 I describe how our results could be used to characterize the neutron background for the EXO experiment. 


\subsection{Error estimation}

All physical quantities, including experimental measurements, are meaningless without estimates of the uncertainties, or errors, associated with them. In this section I describe the uncertainties associated with the measurements presented in this thesis. In the first subsection I describe the statistical components of these uncertainties, which stem from unpredictable and unknown variations in the measuring instruments or environmental conditions. By contrast, systematic uncertainties arise from inaccuracies in the measuring devices, data handling systems, and analysis techniques. I discuss these systematic errors in the second subsection.

The errors for all measurements are summarized in Table 7.1 .

\begin{tabular}{lr} 
Process & Error $(\boldsymbol{\%})$ \\
\hline \hline muon flux (total) & $\mathbf{5}$ \\
\hline$N_{\mu}$ & $1^{\dagger}$ \\
$t$ & 1 \\
$\epsilon_{\mu} \& A$ & 5 \\
$\mu$-induced $\boldsymbol{n}$ yield (total) & $\mathbf{8 4}$ \\
\hline$N_{\mu}$ & $1^{\dagger}$ \\
$N_{n, \mu}$ & $37^{\dagger}$ \\
$(1-Q)$ & 6 \\
$\epsilon_{n}$ (total) & 75 \\
$\quad$ capture position & 18 \\
$\gamma$ energy cuts & 73 \\
$l_{\mu}$ & \multicolumn{2}{c}{6} \\
$\rho_{L S}$ & \multicolumn{2}{c}{5} \\
$\boldsymbol{n}$ flux (total) & $\mathbf{9 0}$ \\
\hline$N_{n, n}-N_{n, \mu}^{\prime}($ total) & $50^{\dagger}$ \\
$\quad N_{n, n}$ & $30^{\dagger}$ \\
$\quad N_{n, \mu}^{\prime}$ & $30^{\dagger}$ \\
$t \quad \epsilon_{n} \& A$ (total) & \multicolumn{2}{c}{1} \\
$\quad$ capture position & 75 \\
$\gamma$ energy cuts & 18 \\
n scatter detection & 73 \\
\hline & 3 \\
\hline
\end{tabular}

Table 7.1: Summary of the errors for all measurements. Those marked with $a^{\dagger}$ are primarily or entirely statistical; all others are systematic. The indented entries contribute to the preceding "total" error. Total errors were found by addition in quadrature. 


\subsubsection{Statistical error}

The statistics of particle interaction counting is well described by the Poisson distribution. For such a distribution with a mean value $\mu$ the variance is simply $\sigma^{2}=\mu$ from which we find the standard deviation to be $\sigma=\sqrt{\mu}$. The estimator for the poisson mean, $\mu$, in an experiment is simply the sample mean, but in a counting experiment the count, $N$, is the only sample taken and therefore is the sample mean. Thus the statistical error for a count of particle interactions is simply the square root of the count, $\sigma=\sqrt{N}$. This gives the error for the muon count in the muon flux and neutron spallation measurements; in both cases the muon count error is less than one percent.

The error on the uncorrelated neutron capture candidate rate needed to be calculated separately because this rate was not measured by simply counting events. This rate was measured by performing a ROOT fit using the Chi-square fit method as described in Sec. 6.6.1. The fit method provided a relative error for this measurement of $0.7 \%$. This error is both

systematic and statistical, but the variation in the data of Fig. 6.13 indicates that it is primarily statistical. This error contributes to the neutron counts for the neutron flux and neutron spallation measurements because the corresponding rate is used in the background subtraction. The resulting neutron count statistical errors were \pm 49 neutrons, $30 \%$, and \pm 39 neutrons, $37 \%$, for the neutron flux and spallation counts respectively. These errors are relatively large due to the large background subtraction.

\subsubsection{Systematic error}

\section{Cosmic muon flux}

The error for the muon flux is effectively only systematic. Following the flux calculation method of Eqn. 7.1, the statistical error in the muon count, $N_{\mu}$, and the systematic error in the live time, $t$, are both less than one percent. The systematic error in the muon efficiency, $\epsilon_{\mu}$, and its corresponding cross sectional area, $A$, were assessed by calculating the flux using 
five distinct methods and comparing the results. Figure 7.1 shows the agreement between these methods as a function of fiducial cross sectional area, or equivalent. The divergence of the methods at lower fiducial cross section is understood as subtle differences between the cross sections of each method for the detection of muons at larger azimuthal angle. The simulation of these higher angled muons did not exactly match the physical spectrum and each of these methods varied slightly in their sensitivity to this imperfection. The "Fiducial (no y)" method additionally had a systematic error because its fiducial volume was not vertically centered on the average position of muon energy deposit. The method thus counted fewer muons than a properly centered fiducial volume would, particularly for smaller fiducials of size comparable to the fiducial volume offset. This offset was present in the other methods as well, but their fiducial cuts were not sensitive to the muon average vertical position. From the spread of these results the systematic error was judged to be $0.04 \mathrm{~m}^{-2} \mathrm{~s}^{-1}$ or $5 \%$.

The five methods can be categorized by two distinctions: what fiducial volume cuts were used in muon counting and how the Monte Carlo simulation was used to calculate the muon detection efficiency. The "Fiducial (w/y)" and "Sphere" methods used the default fiducial cuts as described by the "box method" and "sphere method" of Sec. 6.4.2. The "Fiducial (no y)" method used fiducial cuts according to the "box method" except that no cuts were imposed in the vertical, $y$, direction. The "Plane" method cut on fiducial similarly to the "sphere method" except that it required muon tracks to pass through a horizontal plane instead of a sphere. Since a plane has a fundamentally different cross section than a sphere, the angular distribution proposed by Miyake [13] was used to convert the planar flux into an equivalent spherical flux.

The other defining feature of these five calculation methods is the way in which the simulation was used to assess the detection efficiency. The simulation, described in Sec.6.7.6, was used in its entirety for the "Fiducial" and "Sphere 2" methods. In these cases the ratio of simulated muons detected to the total generated gave the detection efficiency and the 
cross section of the generating sphere was used for the area, $A$. In the case of the "Sphere 1" and "Plane" methods, however, only the muons whose initial trajectory would pass through the fiducial sphere or plane were included in the simulated muon sample. The number of muons detected divided by the number in this sample gave the efficiency for these methods and the cross sectional area of the target sphere or plane was used for $A$.

\section{Muon Flux Vs Fiducial Area (or equivalent)}

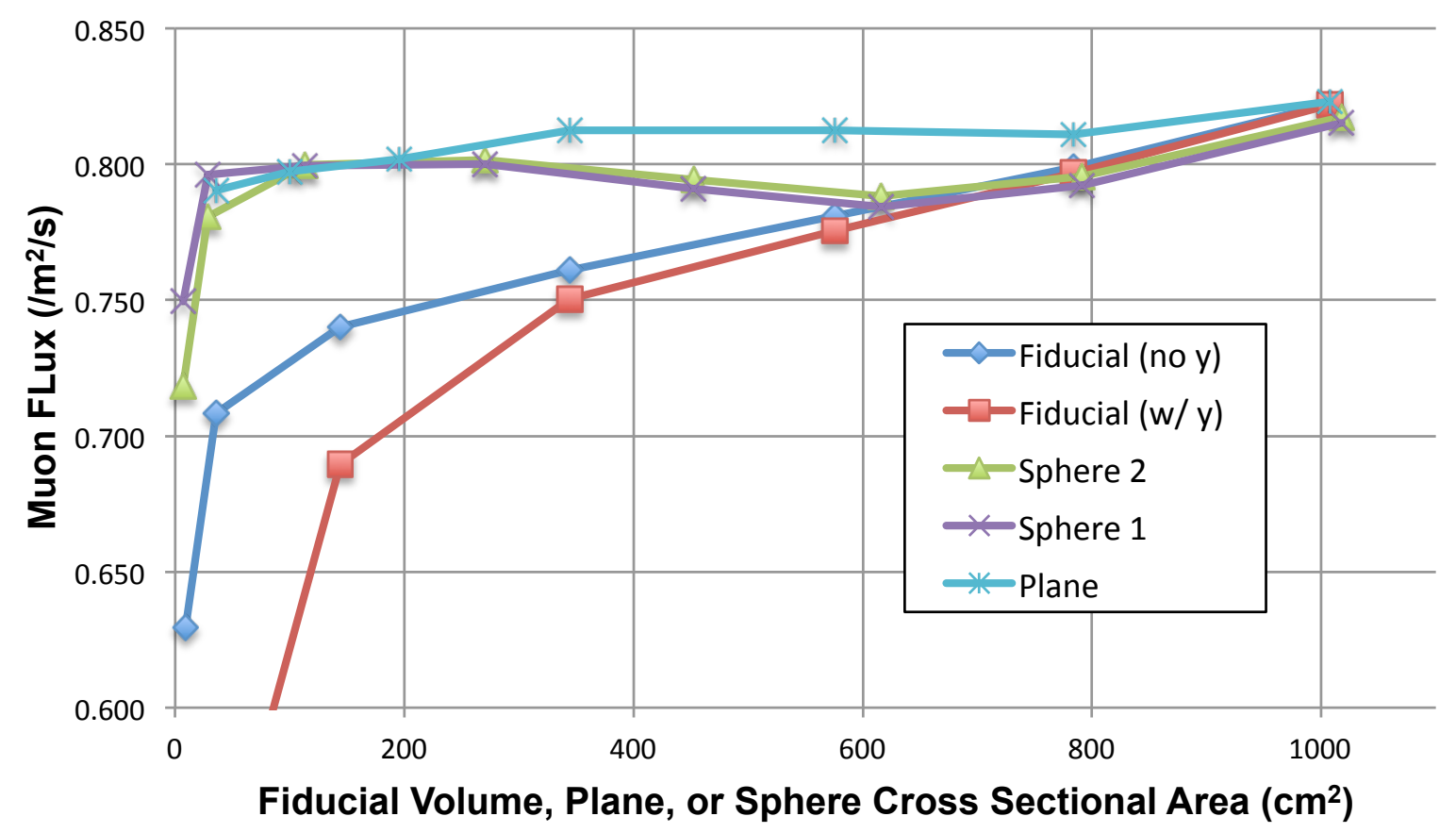

Figure 7.1: Muon fluxes versus effective fiducial cross sectional area for five different methods. The lines drawn simply connect the data points to guide the eye. The "Sphere 2" at $791 \mathrm{~cm}^{2}$ was used for the muon flux result. The drop and divergence in fluxes at small fiducial volumes is understood to be caused by reductions in the SciBath effiective cross sectional area for imperfectly simulated high angle muons, which varies with the fiducial method.

\section{Muon-induced neutron spallation in scintillator}

The error in the neutron spallation in scintillator result has contributions from both statistical and systematic effects. Following the yield calculation of Eqn. 7.2, the error in the neutron and muon counts, $N_{n, \mu}, N_{n, n}$, and $N_{\mu}$, are considered entirely statistical. The error 
on the liquid scintillator density, $\rho_{L S}$, is estimated to be less than $5 \%$. Small variations to the muon simulation were made to assess the error on the muon track length, $l_{\mu}$, to be $6 \%$.

The systematic error in the neutron capture and detection efficiency, $\epsilon_{n}$, were assessed by testing variations in the photon generation position and variations in the energy calibration for photon detection. Section 6.7 .4 describes the Geant4 simulation in which $2.2 \mathrm{MeV}$ photons were generated in the inner $70 \%$ of the detector volume to assess the photon detection efficiency. I recalculated the detection efficiency with a simulation that generated photons uniformly in the entire detector volume, and the difference suggested an error of $18 \%$.

To assess the error in the photon detection efficiency caused by the energy calibration and cuts, our best beam correlated $2.2 \mathrm{MeV}$ photon candidate energy distribution was fit with a Gaussian curve and the portion of the fit curve that passed the energy cuts of Table 6.1 was found. This efficiency for a $2.2 \mathrm{MeV}$ photon to pass the energy cuts was compared to the corresponding efficiency from fitting the simulated photon energy spectrum of Sec. 6.7.4 with a Gaussian. These Gaussian fits can be seen in Figs. 7.2 and 7.3 where the data fit has the largest reasonable mean considering the fact that we expect half or more of the lower energy portion of the distribution to be reduced due to a soft threshold caused by various particle identification cuts. The discrepancy between these two efficiency calculations resulted in a $73 \%$ error on the simulated efficiency. Adding this in quadrature to the position portion of the error gives a total $2.2 \mathrm{MeV}$ photon detection efficiency error of $75 \%$.

The correction factor, $Q$ does not suffer from the large error from gamma detection because that dependence divides out in $\epsilon_{n, A l} / \epsilon_{n, L S}$. The largest contributing error is in the other components of the efficiency to detect a neutron from spallation in the aluminum. Overall, we assess the error in $Q$ to be less than $30 \%$, and therefore the error in $(1-Q)$ is at most $6 \%$.

Adding errors for all terms of Eqn. 7.2 in quadrature I found the final cosmic neutron production in scintillator error to be $84 \%$ or $3.2 \times 10^{-4} \mathrm{n} / \mu\left(\mathrm{g} / \mathrm{cm}^{2}\right)^{-1}$. This calculation is summarized in Table 7.1 . 


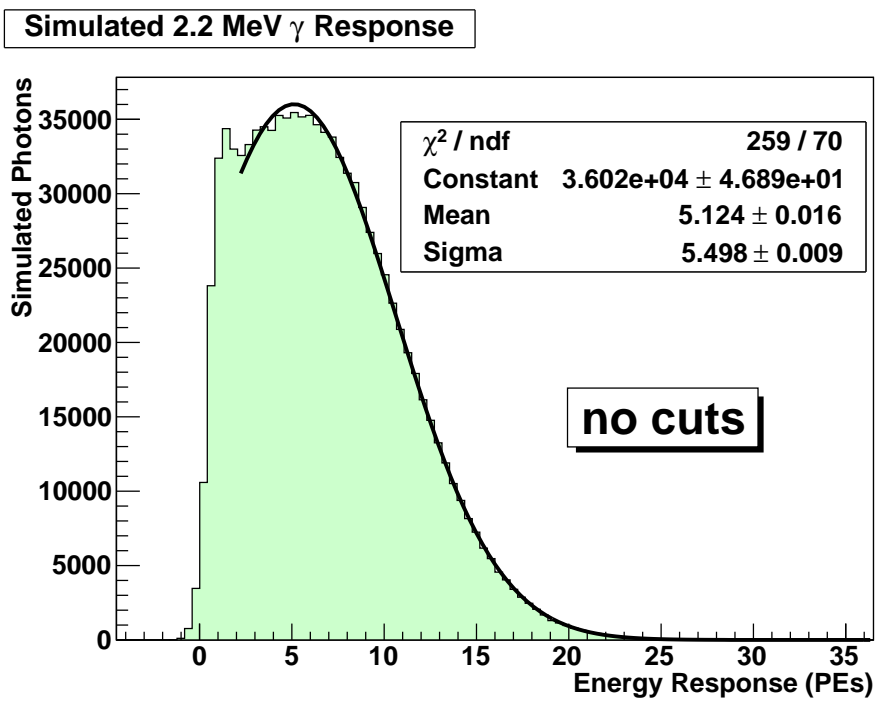

Figure 7.2: Geant4 simulated detector energy response to $2.2 \mathrm{MeV}$ photons.

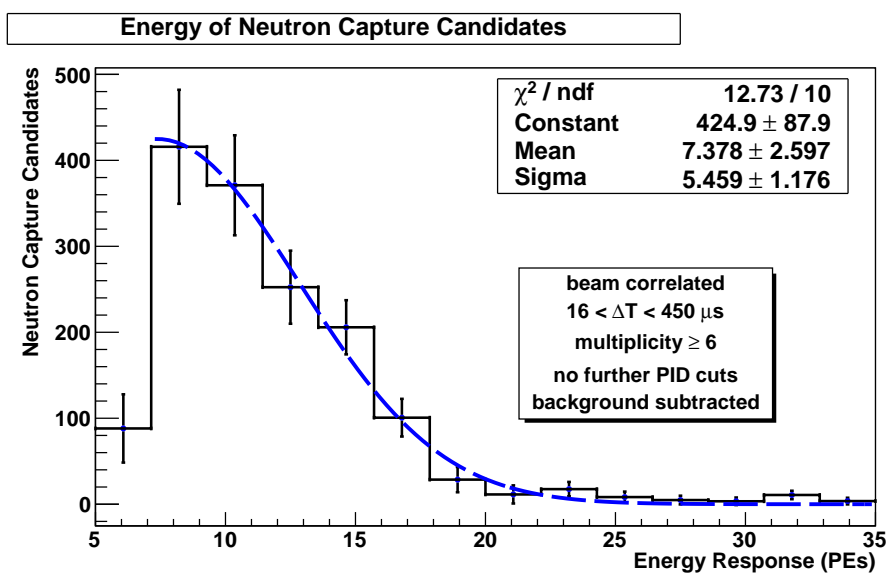

Figure 7.3: Beam correlated 2.2 MeV photon response with the largest reasonable Gaussian fit. The discrepancy between the data and the fit at low PE is due to the soft threshold caused by our particle identification cuts, primarily the multiplicity greater than five requirement. 


\section{Cosmic neutron flux and spectra}

The error in the neutron flux result has contributions from both statistical and systematic effects. Following the flux calculation of Eqn. 7.6, the error in the neutron count, $N_{n, n}$, is entirely statistical and the systematic error in the live time, $t$, is less than one percent. The error in the neutron detection efficiency, $\epsilon_{n}$, results from the neutron capture and $2.2 \mathrm{MeV}$ photon detection efficiencies as well as the neutron scattering detection efficiency. The errors on the neutron capture and photon detection efficiencies are the same as described in the muon induced neutron spallation in scintillator section of Sec. 7.1 .2 , thus the same $75 \%$ error result was used. The error on the neutron scattering detection efficiency was assessed by testing the effects of variations in our energy calibration on the efficiency and resulted in a $3 \%$ error. The error on the neutron production in scintillator correction, $N_{n, \mu}^{\prime}$, is heavily dominated by the $30 \%$ statistical error in the neutron count, $N_{n, \mu}$. The $2.2 \mathrm{MeV}$ photon detection efficiency error divides out in the $\epsilon_{n}^{\prime} / \epsilon_{n}$, as do many of the errors in the other terms. Therefore, the error used for $N_{n, \mu}^{\prime}$ was $30 \%$.

Adding errors for all terms of Eqn. 7.6 in quadrature, I found the final cosmic neutron flux error of $90 \%$ or $1.4 \times 10^{-2} \mathrm{~m}^{-2} \mathrm{~s}^{-1}$. This calculation is summarized in Table 7.1 . The statistical errors apply to the neutron energy spectrum bin-by-bin, but the systematic errors act as a scale on the entire distribution.

\subsection{Cosmic muon flux}

We have measured the cosmic muon flux in the MINOS near detector hall at FNAL to be $0.80 \pm 0.04 \mathrm{~m}^{-2} \mathrm{~s}^{-1}$. The calculation is summarized in Table 7.2 and Eqn. 7.1.

$$
\Phi_{\mu}=\frac{N_{\mu}}{t \cdot \epsilon_{\mu} \cdot A}
$$


Here $N_{\mu}$ is the number of muons counted that pass particle identification cuts of Table 6.1. $t$ is the total live time used in the analysis, $\epsilon_{\mu}$ is the efficiency for muon detection from Sec. 6.7.6 for the "Sphere 2" method, and $A=\pi(0.40 \mathrm{~m})^{2}$ is the cross sectional area for the simulation method used in calculating $\epsilon_{\mu}$.

\begin{tabular}{ccc} 
parameter & value & error (\%) \\
\hline \hline$N_{\mu}$ & 110345 & 1 \\
$t$ & $1457322 \mathrm{~s}$ & 1 \\
$\epsilon_{\mu}$ & 0.1894 & 5 \\
$A$ & $0.50265 \mathrm{~m}^{2}$ & 0 \\
\hline$\Phi_{\mu}$ & $0.80 \mathrm{~m}^{-2} \mathrm{~s}^{-1}$ & 5 \\
\hline
\end{tabular}

Table 7.2: Muon flux calculation summary. The error on $A$ is listed as zero because it is included in the error of $\epsilon_{\mu}$.

Our result is in agreement with those presented in Sec. 3.1, considering their variation. In order to directly compare our result to those plotted in Fig. 3.1, we must multiply by the cube of our depth, 275 m.w.e. from the top of the atmosphere, and divide by the integral of the angular distribution over the upper hemisphere. Integrating the angular spectrum of Eqn. 3.2, which fits our data reasonably well according to Fig. 7.5, and multiplying by the depth cubed scales our result to $754 \pm 38 \mathrm{~cm}^{-2} \mathrm{~s}^{-1} \mathrm{sr}^{-1}$ m.w.e. ${ }^{3}$. By comparison, the curve shown in Fig. 3.1 predicts $808 \mathrm{~cm}^{-2} \mathrm{~s}^{-1} \mathrm{sr}^{-1}$ m.w.e. ${ }^{3}$, just above the error margin of our result. The previous measurements at around 275 m.w.e. also gave a larger flux than our result.

Expressed as a total muon flux, Sec. 3.1 summarized the predictions and previous measurements as $0.86 \pm 0.26 \mathrm{~m}^{-2} \mathrm{~s}^{-1}$. Our result is well within the uncertainty of this prediction and can be use to constrain future predictions at similar depths.

\subsection{Cosmic muon angular distribution}

The angular distribution of muons at the MINOS near detector hall at FNAL was measured by reconstructing muon tracks using the eigenvalue method of Sec. 6.4.3. The muon identification cuts can be found in Table 6.1. Figures 7.4 and 7.5 show the angular distribution 
of detected muons. In the two dimensional plot $\theta$ is measured from the $+z$ direction and $\phi$ is measured in the direction of $+x$ from $+y$. In the one dimensional plot $\theta$ is measured from the $+y$ (vertical) axis. See Fig. 4.3 for the SciBath coordinate system. The one dimensional spectrum is plotted with the parameterization by Miyake for the flux through a point-like detector [13], Eqn. 3.2, which agrees reasonably well with our measurement. The two dimensional spectrum shows uniformity in the azimuthal angle, as expected.

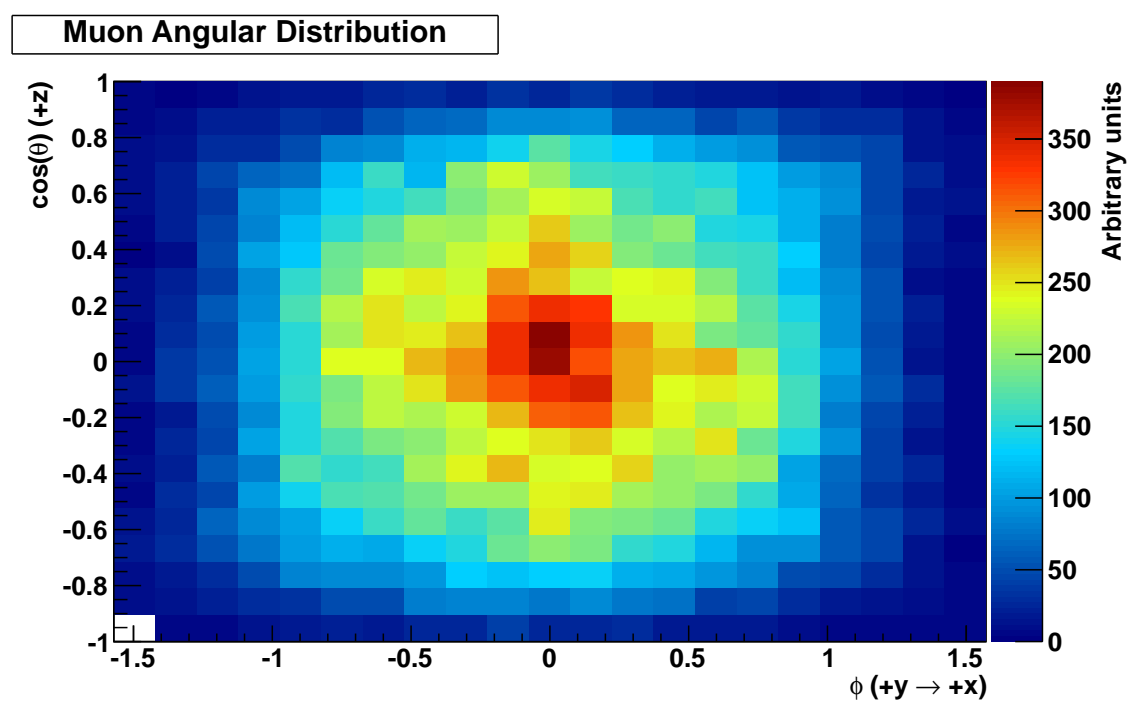

Figure 7.4: Angular distribution of muons at $100 \mathrm{~m}$ underground at the MINOS hall at FNAL.

\subsection{Muon-induced neutron spallation in scintillator}

Muons cause neutron spallation when passing through all forms of matter, including liquid scintillator. The spallation rate, normalized to muon track length and scintillator density, was measured for the NuMI run to be $(3.8 \pm 3.2) \times 10^{-4} \mathrm{n} / \mu\left(\mathrm{g} / \mathrm{cm}^{2}\right)^{-1}$. This measurement was made for two reasons. First, our measurement will contribute to the understanding muon induced neutron spallation in general. Second, muons can pass neutron scatter identification cuts while simultaneously spallating a neutron that then captures inside SciBath. Thus muon spallation contaminates the neutron flux measurement to some degree. 


\section{Muon Angular Distribution}

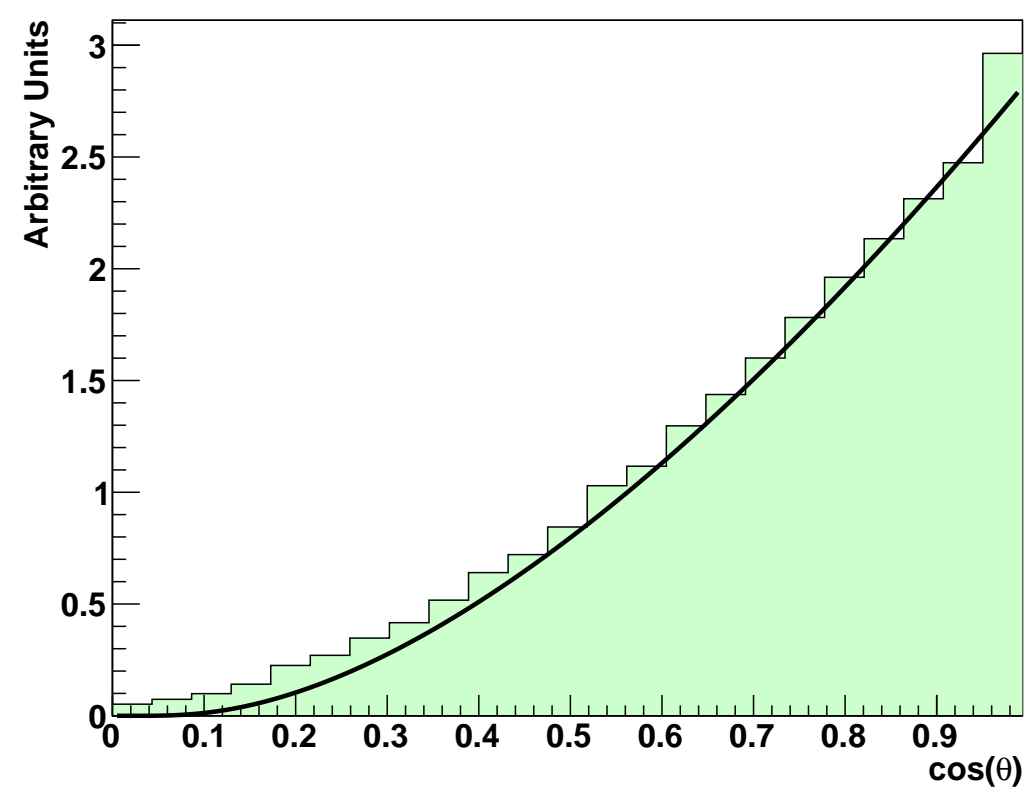

Figure 7.5: Azimuthal angular distribution of muons at $100 \mathrm{~m}$ underground at the MINOS hall at FNAL. The angle $\theta$ is measured from the vertical. The curve drawn is the prediction by Miyake [13].

To measure this production rate, we first counted the total number of muons that passed the muon identification cuts of Table 6.1 without the fiducial cut, $N_{\mu}$. We similarly counted the number of neutron capture candidate events that followed these muons after background subtraction, $N_{n, \mu}$. See Table 6.1 and Sec. 6.2.1 for the specific cuts used for muon and neutron capture candidates. The calculation for the production rate is then described in Table 7.3 and Eqn. 7.2 ,

$$
Y_{n}=\frac{N_{n, \mu} \cdot(1-Q)}{\epsilon_{n} \cdot N_{\mu} \cdot l_{\mu} \cdot \rho_{L S}} .
$$

Here $Q$ is a correction factor to account for neutrons spallated in detector materials instead of scintillator, $\epsilon_{n}$ is the efficiency for a neutron produced inside the scintillator to capture inside the detector and for that capture to generate an event that passes identification cuts, $l_{\mu}$ is the average track length of a muon passing cuts in SciBath, and $\rho_{L S}$ is the density of liquid scintillator. Figure 6.19a provides the average muon track length, $l_{\mu}$, based on simulation. The internally generated neutron detection efficiency, $\epsilon_{n}$, was found in Sec. 6.7.5. 


\begin{tabular}{ccc} 
parameter & value & error (\%) \\
\hline \hline$N_{n, \mu}$ & 105 & 37 \\
$Q$ & 0.16 & 30 \\
$\epsilon_{n}$ & 0.018 & 75 \\
$N_{\mu}$ & 327779 & 1 \\
$l_{\mu}$ & $45.32 \mathrm{~cm}$ & 6 \\
$\rho_{L S}$ & $0.86 \mathrm{~g} / \mathrm{cm}^{3}$ & 5 \\
\hline$Y_{n}$ & $3.8 \times 10^{-4} n / \mu\left(\mathrm{g} / \mathrm{cm}^{2}\right)^{-1}$ & 84 \\
\hline
\end{tabular}

Table 7.3: Muon-induced neutron production calculation summary.

The correction factor $Q$ was estimated by scaling the muon correlated neutron count (this measurement without the $Q$ factor) to an expected number of neutrons from the aluminum walls of the detector cube. Without a correction factor, the neutron yield can be written as

$$
\Phi_{n}=\frac{N_{n, \mu}}{\epsilon_{n} \cdot N_{\mu} \cdot l_{\mu} \cdot \rho_{L S}} .
$$

Previous measurements and simulations suggest that neutron yield scales with target material as $<A>^{\beta}$ [41, 68, 69]. In particular, Formaggio and Martoff suggest $\beta=0.90 \pm 0.23$ [68]. Thus we can use our measurement of neutron production in scintillator to estimate the fraction from the aluminum as follows:

$$
\begin{aligned}
\frac{Y_{n, A l}}{Y_{n, L S}}=\left(\frac{<A_{A l}>}{<A_{L S}>}\right)^{0.90}=\frac{N_{n, \mu, A l} \cdot N_{\mu} \cdot \epsilon_{n, L S} \cdot l_{\mu, L S} \cdot \rho_{L S}}{N_{n, \mu, L S} \cdot N_{\mu} \cdot \epsilon_{n, A l} \cdot l_{\mu, A l} \cdot \rho_{A l}}, \\
Q=\frac{N_{n, \mu, A l}}{N_{n, \mu, L S}}=\left(\frac{<A_{A l}>}{<A_{L S}>}\right)^{0.90} \frac{\epsilon_{n, A l} \cdot l_{\mu, A l} \cdot \rho_{A l}}{\epsilon_{n, L S} \cdot l_{\mu, L S} \cdot \rho_{L S}} .
\end{aligned}
$$

A summary of the calculation can be found in Table 7.4. The walls of the detector cube are 0.5 inches thick, so a vertical muon would pass through 1.0 inch of aluminum (top and bottom). In reality, few muons are vertical. Assuming the Miyake [13] angular distribution, the average muon is angled $38.2^{\circ}$ with respect to the vertical. Depending on the specific geometry of this average muon track passing through the detector cube, it passes through $1.3 \pm 0.1$ inches of aluminum or $3.30 \pm 0.25 \mathrm{~cm}$. 


\begin{tabular}{cc} 
parameter & value \\
\hline \hline$A_{A l}$ & $27 \mathrm{AMU}$ \\
$A_{L S}$ & $10 \mathrm{AMU}$ \\
$\epsilon_{n, A l}$ & 0.0051 \\
$\epsilon_{n, L S}$ & 0.018 \\
$l_{\mu, A l}$ & $3.30 \mathrm{~cm}$ \\
$l_{\mu, L S}$ & $45.32 \mathrm{~cm}$ \\
$\rho_{A l}$ & $2.70 \mathrm{~g} / \mathrm{cm}^{3}$ \\
$\rho_{L S}$ & $0.86 \mathrm{~g} / \mathrm{cm}^{3}$ \\
\hline$Q$ & 0.16 \\
\hline
\end{tabular}

Table 7.4: Muon-induced neutron production correction factor, $Q$, calculation summary.

The calculation for the efficiency for detection of a neutron produced in the aluminum detector walls, $\epsilon_{n, A l}$, is summarized in Table. 7.5. The neutron capture component was estimated by weighting the external neutron capture efficiency of Fig. 6.16 by the Da Silva et al. analytic form for neutron energy from spallation in wax [67] and taking the average. The 0.062 of Sec. 6.7.4 was again used for the efficiency to detect the $2.2 \mathrm{MeV}$ photon from this capture as well as 0.68 from the same section as the efficiency for this capture to pass the timing cuts. Additionally a 0.50 efficiency was added to account for the fact that about half of the neutrons generated in the aluminum will be directed away from the liquid scintillator volume.

\begin{tabular}{cc} 
Process & Efficiency \\
\hline \hline neutron capture & 0.24 \\
$2.2 \mathrm{MeV} \gamma$ detection & 0.062 \\
$\Delta \mathrm{T}$ cuts & 0.68 \\
$\mathrm{n}$ enter detector & 0.50 \\
\hline$\epsilon_{n, A l}$ & 0.0051 \\
\hline
\end{tabular}

Table 7.5: Summary of the detection efficiency for neutrons generated in the detector walls.

To compare our final result of $(3.8 \pm 3.2) \times 10^{-4} n / \mu\left(\mathrm{g} / \mathrm{cm}^{2}\right)^{-1}$ with previous measurements, we calculated the mean muon energy at NuMI with Eqn. 3.7, from Mei and Hime [14]. Table 3.2 is reproduced here as Table 7.6 with an added column for the NuMI result at 265 m.w.e. This gives two predictions for the mean muon energy, both with accepted param- 
eterizations as given in the table. Taking the average of the two predictions gives $37 \pm 6 \mathrm{GeV}$, where the the error is includes the difference between the mean and the predicted mean from either of the given parameterizations, as well as the uncertainty in the depth described in Sec. 5.1.

\begin{tabular}{|c|c|c|c|c|c|}
\hline Ref. & $\epsilon_{\mu}(\mathrm{GeV})$ & $b\left(\mathrm{~km} . \mathrm{w} . \mathrm{e}^{-1}\right)$ & $\gamma_{\mu}$ & $\bar{E}_{\mu}(\mathrm{GeV}, \mathrm{WIPP})$ & $\bar{E}_{\mu}(\mathrm{GeV}, \mathrm{NuMI})$ \\
\hline 45 & 618 & 0.383 & 3.7 & 165 & 35.1 \\
\hline [1, 16 & 693 & 0.4 & 3.77 & 184 & 39.4 \\
\hline
\end{tabular}

Table 7.6: Mean muon energy at WIPP and NuMI and parameters relevant to their calculation with Eqn.3.7

Our neutron production measurement is substantially larger than the previous measurements would predict, though it is in agreement with its relatively large error. Table 7.7 is a reproduction of Table 3.1 with the addition of our result in the fourth row. Similarly, Fig. 7.6 is a reproduction of Fig. 3.3 with the addition of our result. More specific to the NuMI mean muon energy, Eqn. 3.3 predicts a neutron yield of $0.66 \times 10^{-4} \mathrm{n} / \mu\left(\mathrm{g} / \mathrm{cm}^{2}\right)^{-1}$ for $\bar{E}_{\mu}=37 \mathrm{MeV}$ and liquid scintillator, $A=10$. This is a discrepancy of $83 \%$ from our measurement. The relatively large error on our measurement primarily results from the efficiency for 2.2 MeV photons from neutron capture to pass our energy cuts, see Sec. 7.1.2.

\begin{tabular}{ccccc}
$\overline{\boldsymbol{E}}_{\boldsymbol{\mu}}(\mathbf{G e V})$ & $\mathbf{H}(\mathbf{m . w . e .})$ & $\boldsymbol{Y}_{\boldsymbol{L S}}\left(\times \mathbf{1 0}^{-\mathbf{4}} \mathbf{n} \boldsymbol{\mu} \boldsymbol{\mu}\left(\mathbf{g} / \mathbf{c m}^{\mathbf{2}}\right)^{\mathbf{- 1}}\right)$ & year & Ref. \\
\hline \hline $13.0 \pm 7.2$ & 20 & $0.20 \pm 0.07$ & 1995 & {$[33]$} \\
$16.5 \pm 8.1$ & 32 & $0.36 \pm 0.03$ & 2000 & {$[34]$} \\
$16.7 \pm 8.2$ & 25 & $0.36 \pm 0.05^{\dagger}$ & 1973 & {$[35]$} \\
$37 \pm 6$ & 265 & $3.8 \pm 3.2$ & - & this work \\
$86 \pm 18$ & 316 & $0.93 \pm 0.12^{\dagger}$ & 1973 & {$[35]$} \\
$125 \pm 22$ & 570 & $1.57 \pm 0.24^{\dagger}$ & 1986 & {$[36]$} \\
$260 \pm 8$ & 2700 & $2.8 \pm 0.3$ & 2010 & {$[37]$} \\
$280 \pm 33$ & 3100 & $3.3 \pm 0.5^{\dagger}$ & 2005 & {$[38]$} \\
$280 \pm 33$ & 3100 & $3.2 \pm 0.2$ & 2011 & {$[39]$} \\
$385 \pm 39$ & 5200 & $4.1 \pm 0.6^{\dagger}$ & 1989 & {$[40]$} \\
\hline
\end{tabular}

Table 7.7: World measurements of neutron yield in liquid scintillator, including this result. The ${ }^{\dagger}$ indicates measurements that were altered from the original by Ref. [2].

One effect that may contribute to our larger reported neutron yield is the contribution 


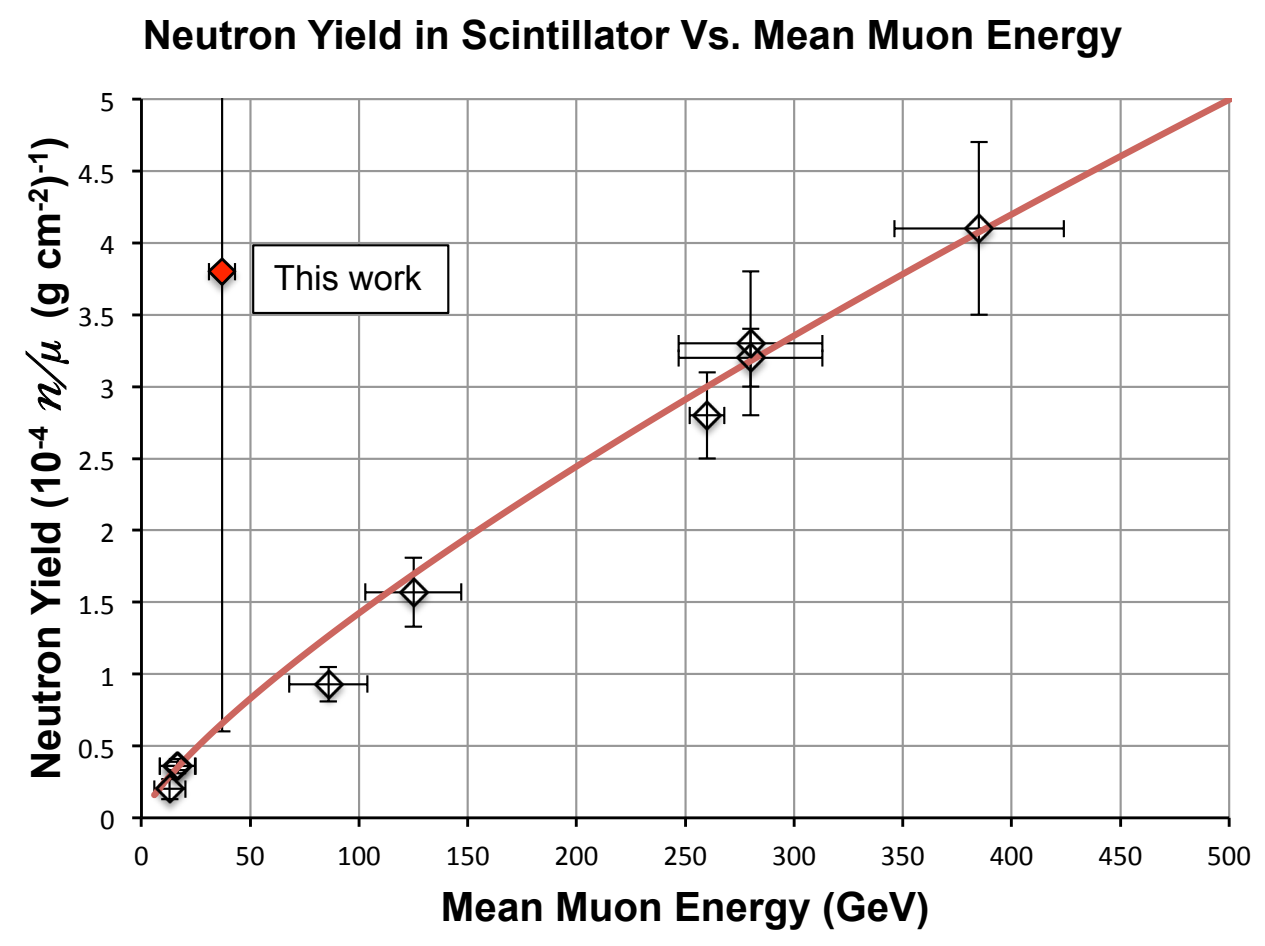

Figure 7.6: World data for neutron production in scintillator, including our result. The curve was proposed by Agafonova and Malgin and is reproduced in Eqn. 3.3. See Table 3.1 for references and identification of which results have been corrected by Agafonova and Malgin. 
from muon capture in the detector. The topology for muon capture events is fundamentally different from muon spallation because the captured muons have shorter tracks. This process was not simulated as part of this analysis, so its effect on the detection efficiency for muoninduced neutron detection is unclear. Figure 2.4 predicts the muon capture contribution to be $11 \%$ of the total neutron yield at 265 m.w.e. Presumably, our analysis counted a portion of neutrons produced through muon capture events. Therefore, we expect the inclusion or exclusion of these neutrons to have at most a $10 \%$ effect. The paper presenting the previous measurement by Boehm et al. [34] does not directly address this contribution, so it is unclear whether their result includes neutrons from muon capture. The paper by Malgin and Ryazhskaya [7] discusses this process in detail and suggests that the previous neutron yield measurements, and Eqn. 3.3, do not include the muon capture contribution.

Another effect that may contribute to our larger reported neutron yield is that of muon induced showers outside of the detector, which may cause multiple particles to enter the detector along with the muon. If one of these accompanying particles was a neutron that later captured inside the detector, the neutron production would have been misidentified as occurring inside the detector. These showers would therefore inflate our result. This effect is discussed in Refs. [2, 70, 34, 33], where Agafonova and Malgin [2] used the results of Zbiri [70] regarding the effect to correct some of the measurements in Table 7.7. The size of the effect is geometry dependent, especially at shallow depths where the muon angular spectrum is less vertical. The simulation by Zbiri [70] suggests a roughly $30 \%$ effect, especially for deeper sites. Boehm et al. [34] give a roughly $18 \%$ effect on their result while Hertenberger et al. 33] describe a $60 \%$ reduction in their result; both at shallow depth. If the effect on our measurement is as large as it was for Hertenberger et al., then this effect would account for the majority of the discrepancy between our result and the previous measurements and simulations. 


\subsection{Cosmic neutron flux}

The cosmogenic neutron flux in the MINOS near detector hall at FNAL was measured to be $(1.5 \pm 1.4) \times 10^{-2} \mathrm{~m}^{-2} \mathrm{~s}^{-1}$. The calculation is summarized in Table 7.8 and Eqn. 7.6 .

$$
\Phi_{n}=\frac{N_{n, n}-N_{n, \mu}^{\prime}}{t \cdot \epsilon_{n} \cdot A}
$$

Here $N_{n, n}$ is the number of correlated neutron scatter-capture pairs that passed particle identification cuts of Table 6.1 and timing cuts of Sec.6.2.1. The total live time used in this analysis is denoted $t$ and the cross sectional area for the detector for the simulation method used is denoted $A=\pi(0.40 \mathrm{~m})^{2}$. The efficiency to detect neutrons by this method, $\epsilon_{n}$, had five contributions and is described by the simulation results of Sec. 6.7.4.

\begin{tabular}{ccc} 
parameter & value & error (\%) \\
\hline \hline$N_{n, n}$ & 166 & 30 \\
$N_{n, \mu}^{\prime}$ & 60 & 30 \\
$t$ & $3519637 \mathrm{~s}$ & 1 \\
$\epsilon_{n}$ & 0.0039 & 75 \\
$A$ & $0.5027 \mathrm{~m}^{3}$ & 0 \\
\hline$\Phi_{n}$ & $1.5 \times 10^{-2} \mathrm{~m}^{-2} \mathrm{~s}^{-1}$ & 90 \\
\hline
\end{tabular}

Table 7.8: Neutron flux calculation summary. The error on $A$ is listed as zero because it is included in the error of $\epsilon_{n}$.

$N_{n, \mu}^{\prime}$ is the expected number of muons that were identified as neutron scattering events that also spallated a neutron whose capture was detected, thus passing neutron identification cuts. This correction was assessed using the neutron production rate from muon spallation in liquid scintillator described in Sec. 7.4. Since this muon contamination is from the same spallation process it is described by Eqn. 7.3, and we can write

$$
\begin{gathered}
\frac{N_{n, \mu}}{N_{\mu} \cdot \epsilon_{n} \cdot l_{m u} \cdot \rho}=Y_{n}=Y_{n}^{\prime}=\frac{N_{n, \mu}^{\prime}}{N_{\mu}^{\prime} \cdot \epsilon_{n}^{\prime} \cdot l_{m u}^{\prime} \cdot \rho}, \\
N_{n, \mu}^{\prime}=\frac{N_{n, \mu} \cdot N_{\mu}^{\prime} \cdot \epsilon_{n}^{\prime} \cdot l_{m u}^{\prime}}{N_{\mu} \cdot \epsilon_{n} \cdot l_{m u}},
\end{gathered}
$$


where $Y_{n}^{\prime}$ is the neutron yield from muons that pass neutron cuts. Here the scintillator density, $\rho$, has been cancelled since it is the same for both samples. A summary of the parameters used in the calculation is presented in Table 7.9. The average muon track length and muon and neutron counts for the neutron production measurement were found in Sec. 7.4. To determine the number and corresponding track length of contaminating muons, the simulated muon track length distributions in Fig. 6.19 were used. Dividing the 393852 muons passing muon identification cuts by the 1000000 muons simulated, we found that we detect $39.4 \%$ of the muons that enter the detector. To find the fraction of these that pass neutron identification cuts, the neutron identification analysis was performed on the muon simulation that produced Fig. 6.19. This was done in two samples because the eigenvalue cuts were different above and below 250 PEs. The resulting fraction of the simulated muons detected in each regime provided an estimate of the number of muons passing neutron scattering identification cuts. Additionally, the simulation provided the track lengths for these muons in each regime, as shown in Fig. 6.19.

\begin{tabular}{lr} 
parameter & value \\
\hline \hline$N_{n, \mu}$ & 105 \\
$N_{\mu}$ & 327779 \\
$l_{\mu}$ & $45.32 \mathrm{~cm}$ \\
\hline $\mathrm{PE}>250$ & \\
$N_{\mu}^{\prime}$ & 31891 \\
$\epsilon_{n}^{\prime} / \epsilon_{n}$ & 0.835 \\
$l_{\mu}^{\prime}$ & $38.46 \mathrm{~cm}$ \\
$\mathbf{N}_{\mathbf{n}, \mu}^{\prime}$ & $\mathbf{7}$ \\
$\mathrm{PE}<250$ & \\
$N_{\mu}^{\prime}$ & 346037 \\
$\epsilon_{n}^{\prime} / \epsilon_{n}$ & 0.682 \\
$l_{\mu}^{\prime}$ & $31.99 \mathrm{~cm}$ \\
$\mathbf{N}_{\mathbf{n}, \mu}^{\prime}$ & $\mathbf{5 3}$ \\
\hline $\mathbf{N}_{\mathbf{n}, \mu}^{\prime}$ (total) & $\mathbf{6 0}$ \\
\hline
\end{tabular}

Table 7.9: Neutron flux correction, $N_{n, \mu}^{\prime}$, calculation summary.

The only remaining factor needed to calculate $N_{n, \mu}^{\prime}$ is $\epsilon_{n}^{\prime} / \epsilon_{n}$, the ratio of the efficiencies for neutron detection for neutron-like muon spallated neutrons to the standard internal neutron 
detection efficiency described in Sec. 7.4. The neutron production process is the same in both cases, but since muons identified as neutron scattering events have lower energies and shorter track lengths, they are closer to the edges of the detector, and so the produced neutrons are more likely to escape. This difference was assessed by taking the average of the neutron capture efficiency in Fig. 6.18, weighted by the average reconstructed position of simulated muons in Fig. 6.20. Theses averages were 0.1922, 0.1604, and 0.1311 for muons passing muon identification cuts, muons passing neutron identification cuts above 250 PEs, and muons passing neutron identification cuts below $250 \mathrm{PEs}$, respectively. Thus the ratio of the neutron efficiencies is $\epsilon_{n}^{\prime} / \epsilon_{n}=0.1604 / 0.1922=0.835$ and $\epsilon_{n}^{\prime} / \epsilon_{n}=0.1311 / 0.1922=0.682$ for contaminating muons above and below 250 PEs, respectively.

Carrying out the calculation for $N_{n, \mu}^{\prime}$ gives 7 contaminating muons above 250 PEs and 53 below 250 PEs. The total number of contaminating muons, $N_{n, \mu}^{\prime}$, is then the sum of these: 60 .

Our final result of $(1.5 \pm 1.4) \times 10^{-2} \mathrm{~m}^{-2} \mathrm{~s}^{-1}$ is high compared to the prediction by Mei and Hime [14], given in Eqn. 3.4. One should note that this prediction was not intended to include such shallow depths and its extrapolation may not be very trustworthy. Nevertheless, the prediction suggests a neutron flux of $9.5 \times 10^{-3} \mathrm{~m}^{-2} \mathrm{~s}^{-1}$ at $265 \mathrm{~m}$.w.e. This predicted result does not directly compare with ours, however, because it includes neutrons of all energies. The Mei and Hime predicted energy distribution of Eqn. 3.5 and their results in Table $\mathrm{V}$ of Ref. [14] were used to reduce the flux to only that expected between 16 and $156 \mathrm{MeV}$. This gives a predicted neutron flux of $1.3 \times 10^{-3} \mathrm{~m}^{-2} \mathrm{~s}^{-1}$, which is discrepant with our result by $1.4 \times 10^{-2} \mathrm{~m}^{-2} \mathrm{~s}^{-1}$, matching our stated error.

To compare our result with those of others, a discussion of convention is required. Our result is a neutron flux in an underground cavern per unit cross-sectional area of a perfect spherical detector inside that cavern. This is the "total flux" definition as used for muons. An alternative convention for the flux definition is that through a unit surface area of the rock-cavern boundary. Mei and Hime do not clearly state which convention they use for their 
neutron flux predictions in Ref. [14]. To express our result according to the second convention we must multiply by the ratio of the simulated cross sectional area to the generation surface area: $1 / 4$. Our result with this convention is then $(3.8 \pm 3.4) \times 10^{-3} \mathrm{~m}^{-2} \mathrm{~s}^{-1}$, which is much less discrepant with the Mei and Hime prediction.

The misidentified external muon showers discussed at the end of Sec. 7.4 would have two effects on our neutron flux result. First, since accounting for the effect would reduce our measured muon-induced neutron production rate, it would also reduce the expected number of muon spallation events misidentified as neutrons, $N_{n, \mu}^{\prime}$, see Eqn. 7.7. Second, the neutrons from these external muon showers are precisely what this neutron flux is measuring. Therefore, any neutrons from the external showers should be counted toward the neutron count, $N_{n, n}$; currently they are not. Both of these influences of external showers act to increase our measured neutron flux. For example, if the external shower effect was large enough to bring our neutron production result down to the $0.66 n / \mu\left(\mathrm{g} / \mathrm{cm}^{2}\right)^{-1}$ predicted by Eqn. 3.3 , then our neutron flux measurement would rise to $3.5 \times 10^{-2} \mathrm{~m}^{-2} \mathrm{~s}^{-1}$.

\subsection{Cosmic neutron energy and time distributions}

This analysis identified neutrons by requiring neutron scatter and capture events in coincidence. To verify that this neutron signal was identified correctly, the time difference distribution between neutron scattering and capture candidates, $\Delta \mathrm{T}$, was compared to the expected exponential decay fit of Fig. 7.13. This comparison is shown for cosmogenic neutrons in Fig. 7.7, where reasonable agreement within the relatively large statistical uncertainty can be seen. Figure 7.8 shows the energy distribution for the corresponding neutron scattering events. Since all neutron energy was assumed to be detected through proton recoil from neutron-hydrogen elastic scattering, the quenching analysis of Sec. 6.3 .2 was applied to the energy scale. Also shown is the neutron energy prediction by Mei and Hime [14], Eqn. 3.5 . for WIPP depth, normalized to their predicted neutron flux at NuMI. The spectra shape is 
in reasonable agreement, though our measured flux is higher as stated in Sec. 7.5.

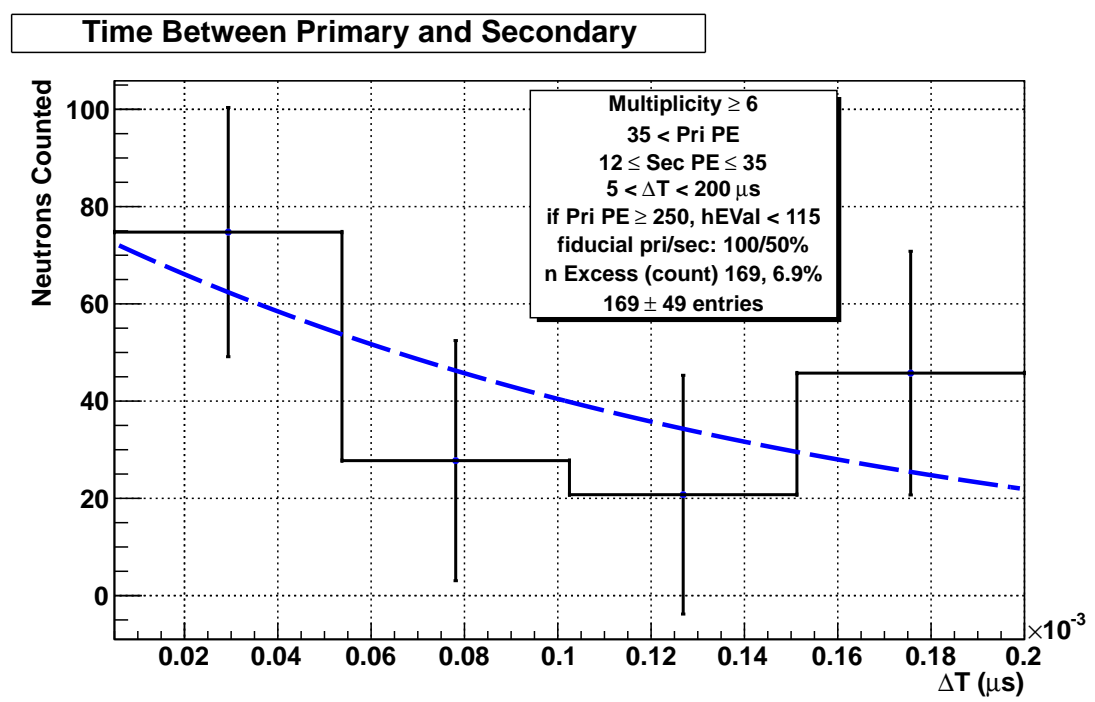

Figure 7.7: Distribution of the time between neutron primary (scatter) and secondary (capture) events. The curve is a decaying exponential of characteristic time $\tau=163 \mu \mathrm{s}$, which is the time structure we expect for neutron capture in SciBath. The distribution is normalized to the number of neutrons counted.

\section{7 neutron / muon ratio}

Since fast neutrons (greater than $16 \mathrm{MeV}$ ) underground are nearly exclusively created by muons, it is convenient to describe the neutron flux as a neutron:muon ratio. Dividing the neutron flux by the muon flux, our measurements give a result of $(1.9 \pm 1.7) \times 10^{-2}$ neutrons per muon in the MINOS near detector hall at FNAL. Figure 7.9 shows the neutron energy spectrum normalized to this ratio.

\subsection{Validation: beam correlated analysis}

During data-taking SciBath was positioned $5 \mathrm{mrad}$ off axis of the NuMI beam, see Sec. 5.2 . Since data was recorded continuously, beam correlated particle tracks were recorded along with the cosmogenic particles. A pre-beam trigger was included in our data stream, allowing 


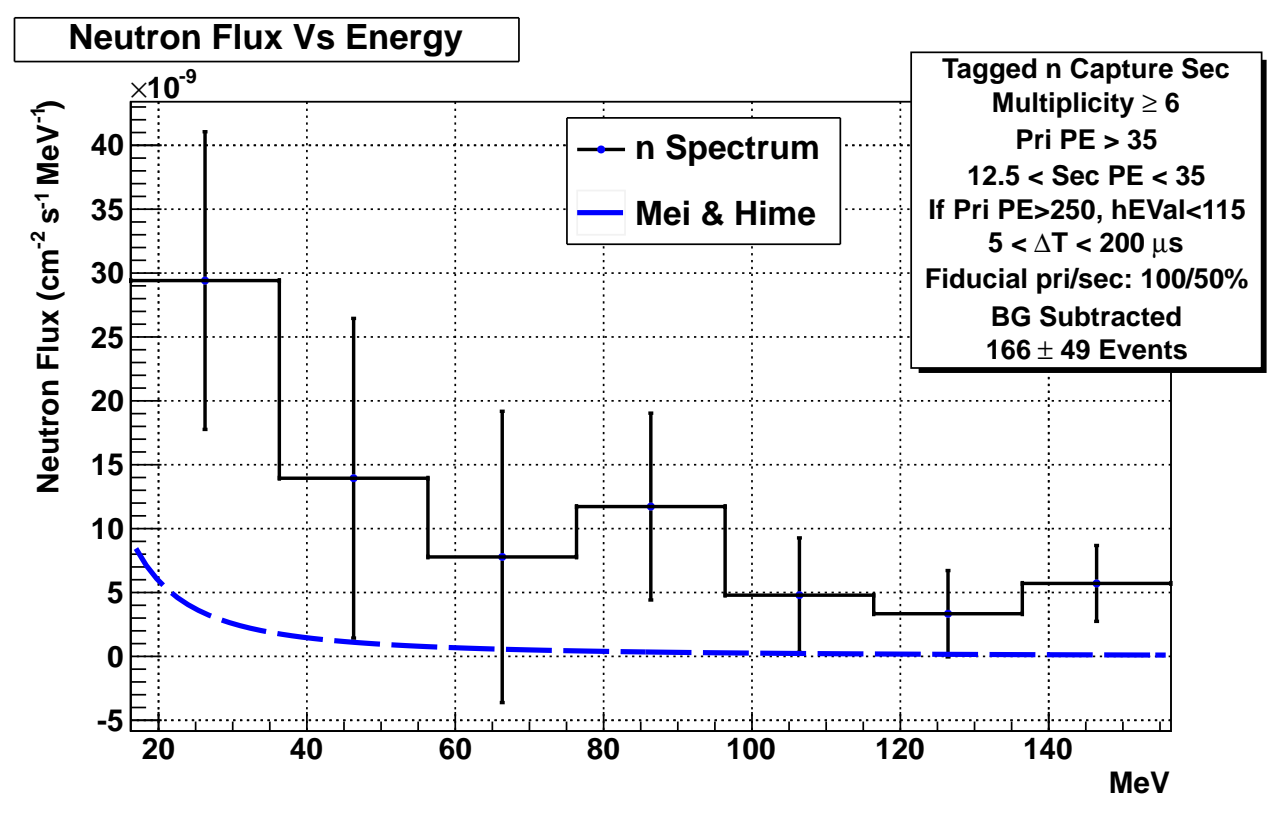

Figure 7.8: Neutron energy spectrum at $100 \mathrm{~m}$ underground at the MINOS hall at FNAL, normalized to the measured flux. The prediction is an extrapolation of the results of Mei and Hime [14]. The errors shown are the statistical errors associated with the background subtracted neutron count, $N_{n, n}$. The systematic errors apply to scale the entire distribution.

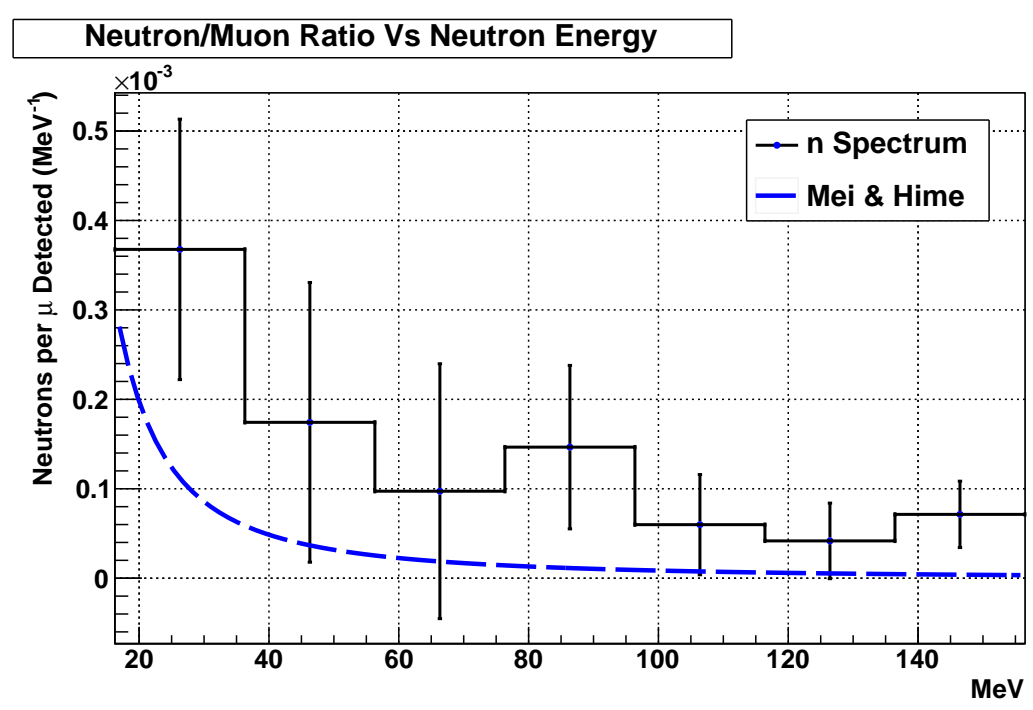

Figure 7.9: Neutron energy distribution normalized to the ratio of the measured neutron and muon fluxes. The prediction is an extrapolation of the results of Mei and Hime [14]. The errors shown are the statistical errors associated with the background subtracted neutron count, $N_{n, n}$. The systematic errors apply to scale the entire distribution. 
us to examine (or exclude) beam correlated events. The beam provided a large sample of neutron and muon interactions with a lower background than the cosmic (beam uncorrelated) data sample. This lower background resulted from the precise timing of the beam pulses. Measurements of neutron and muon characteristics were thus easier for beam correlated data, and these measurements were used to validate the analysis methods used for the cosmic analysis.

Figure 7.10 shows a timing histogram of all events above 10 PEs and within $10 \mu$ s of the beam. The beam structure is clearly visible between 214 and 224 us as well as a significant number of delayed events above background in the microseconds following. Figure 7.11 shows a similar timing distribution, but three histograms are plotted, each giving the event rate for different candidate particles. The beam structure is clear for events of each type as well as evidence of delayed neutron scatters and captures with the expected time structure following the beam.

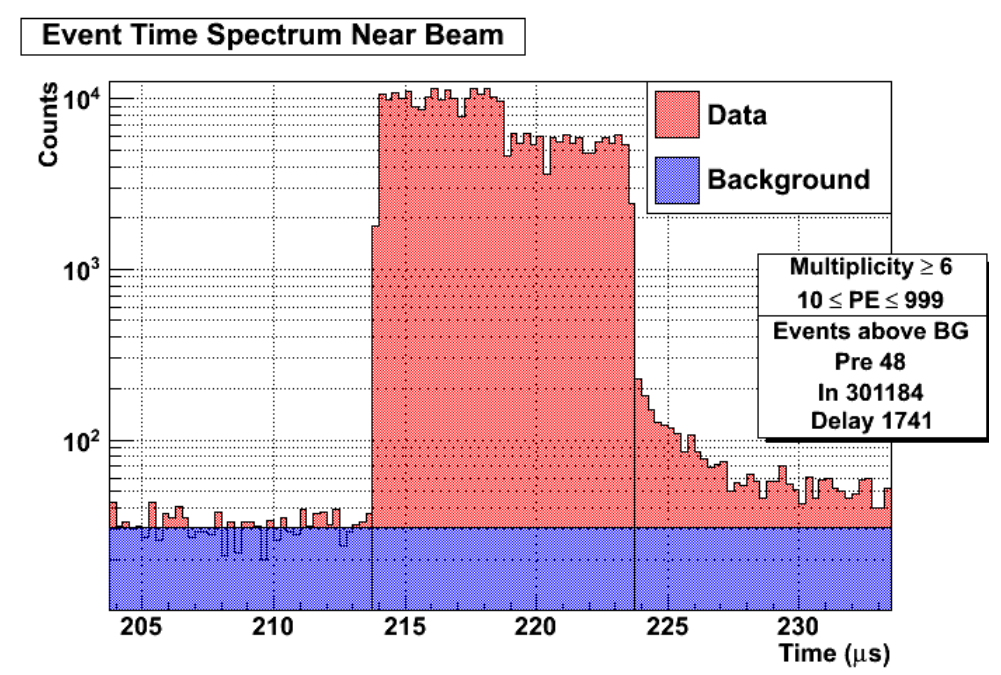

Figure 7.10: The SciBath integrated detector response before, during, and after the NuMI beam pulse.

The same analysis described for cosmic muons in Sections 7.2 and 7.3 was applied to beam correlated muons. The only changes in the analysis were the requirement that events occur during the beam pulse and different fiducial cuts. For the muon flux measurement a 50\% 


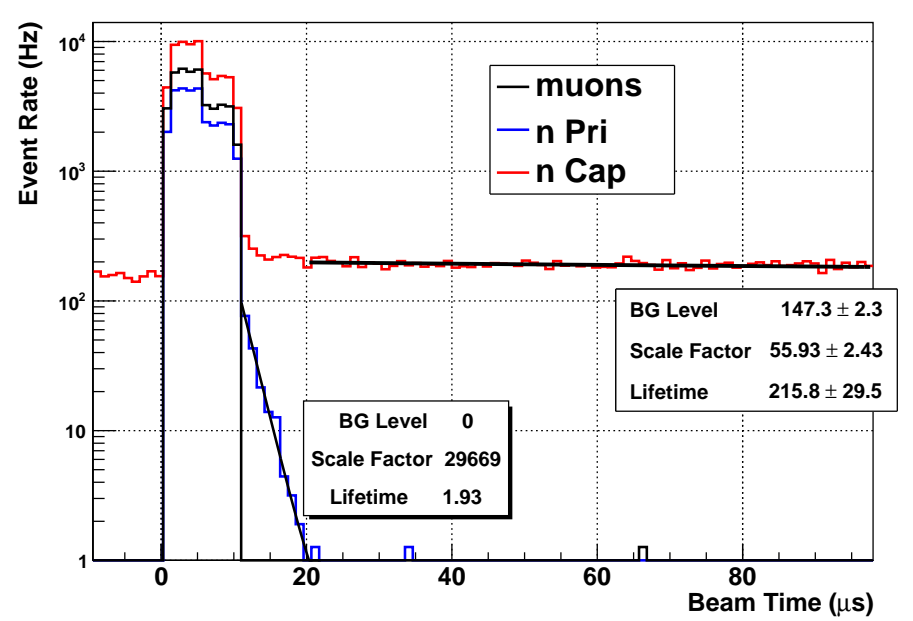

Figure 7.11: Event rate before, during, and after the NuMI beam pulse for muon-, neutron scatter-, and neutron capture-like events. The neutron scatter and neutron capture candidate events are fit with decaying exponentials after the beam pulse.

fiducial volume cut with the box method was used instead of the $26 \%$ with the sphere method described in Table 6.1. The beam correlated muon flux was measured to be $0.46 \pm 0.04 \mathrm{~m}^{-2}$ per beam spill. For the angular distribution in Fig. 7.12 a $10 \%$ fiducial volume with the box method was used. This tighter fiducial allowed a more precise angular reconstruction. Details regarding the beam-correlated muon analysis can be found in an internal SciBath technote by Lori Rebenitsch [71].

Figure 7.13 shows our best fit of the neutron capture time structure. To maximize statistical power, a primary event was not required. Instead, the plot shows all neutron capture candidate events following a beam pulse. The fit of this distribution gave the best value of the neutron capture mean time as $\tau=163 \pm 12 \mu \mathrm{s}$, where the error was found by the ROOT fit.

The same analysis described for cosmic neutrons in Sec. 7.5 was applied to beam correlated neutrons. The only change in the analysis was the requirement that neutron scatter candidates occur during the beam pulse and neutron capture candidates occur in roughly the following $450 \mu \mathrm{s}$ as described in Sec.6.2.2. The beam correlated neutron flux was measured 

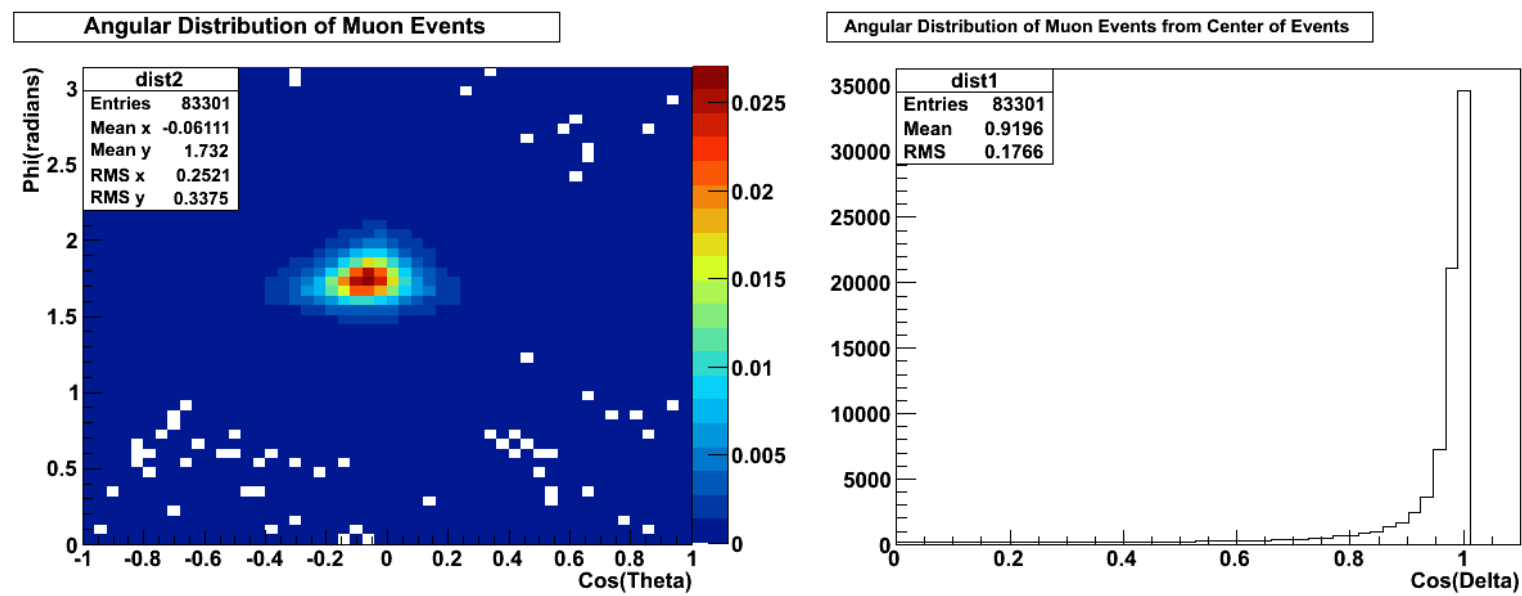

Figure 7.12: Angular distribution of NuMI beam correlated muons at the MINOS hall at FNAL. The angle in the right plot is measured from the beam direction.

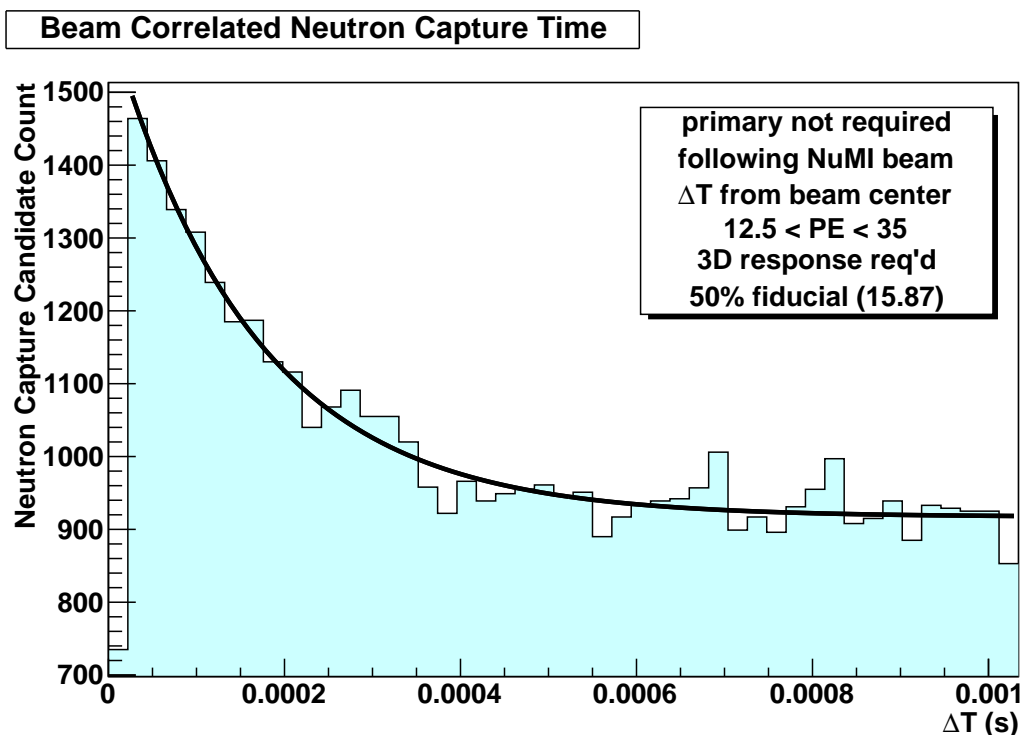

Figure 7.13: Background subtracted distribution of the time between the beam center and following neutron capture candidate events. The curve is the fitted decaying exponential that resulted in the $\tau=163 \mu \mathrm{s}$ characteristic time used in the cosmic analysis. 
to be $0.11 \pm 0.09 \mathrm{~m}^{-2}$ (beam spill) ${ }^{-1}$. This result assumes the neutron detection efficiency described in Sec. 6.7.4 and does not assess possible contamination from muon-induced spallation. Figure 7.14 shows the time spectrum between beam correlated neutron scatter and capture events. The dashed curve drawn is a prediction based on the number of events counted and an exponential decay of mean 163 us. Figures 7.15 and 7.16 show the neutron scatter and capture energy distributions, respectively, where the primary spectrum is normalized to the measured flux.

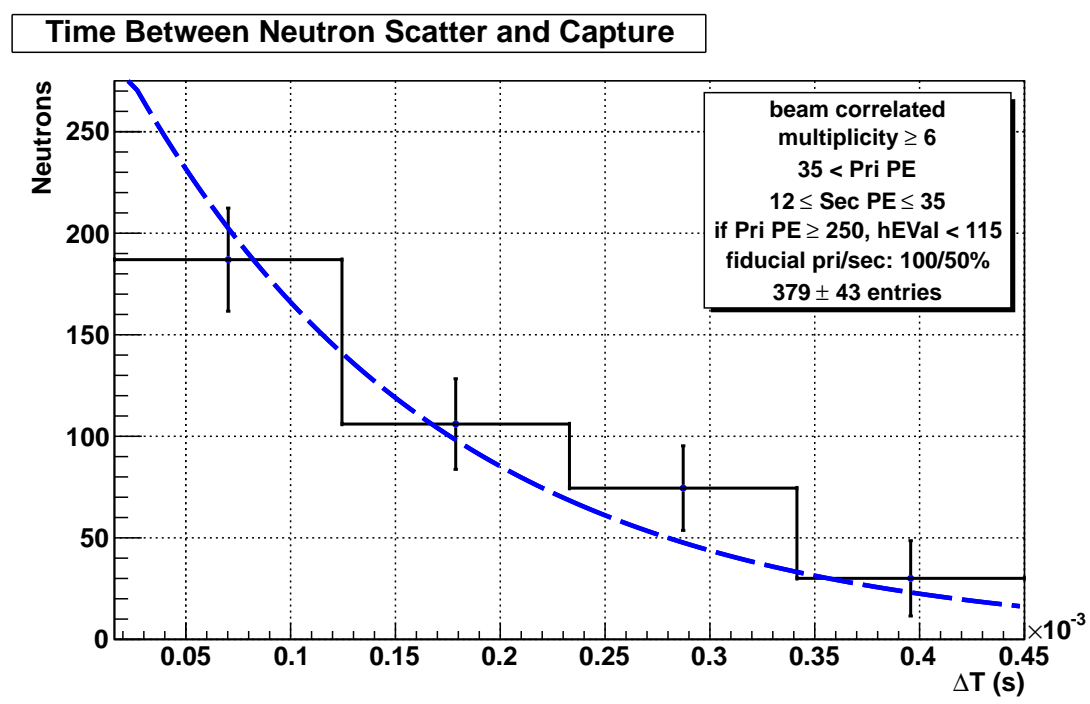

Figure 7.14: Background subtracted distribution of the time between neutron primary (scatter) and secondary (capture) beam correlated events. The curve is a decaying exponential of characteristic time $\tau=163 \mu \mathrm{s}$, which is the time structure we expect for neutron capture in SciBath.

\subsection{Implications for EXO}

The Enriched Xenon Observatory (EXO) [72] is a proposed ton-scale liquid xenon detector that would search for neutrinoless double beta decay. Beta decay is the process by which a neutron decays into a proton, an electron, and an electron neutrino as shown in Eqn. 7.9.

$$
\mathrm{n} \rightarrow p+\mathrm{e}^{-}+\bar{\nu}_{e}
$$




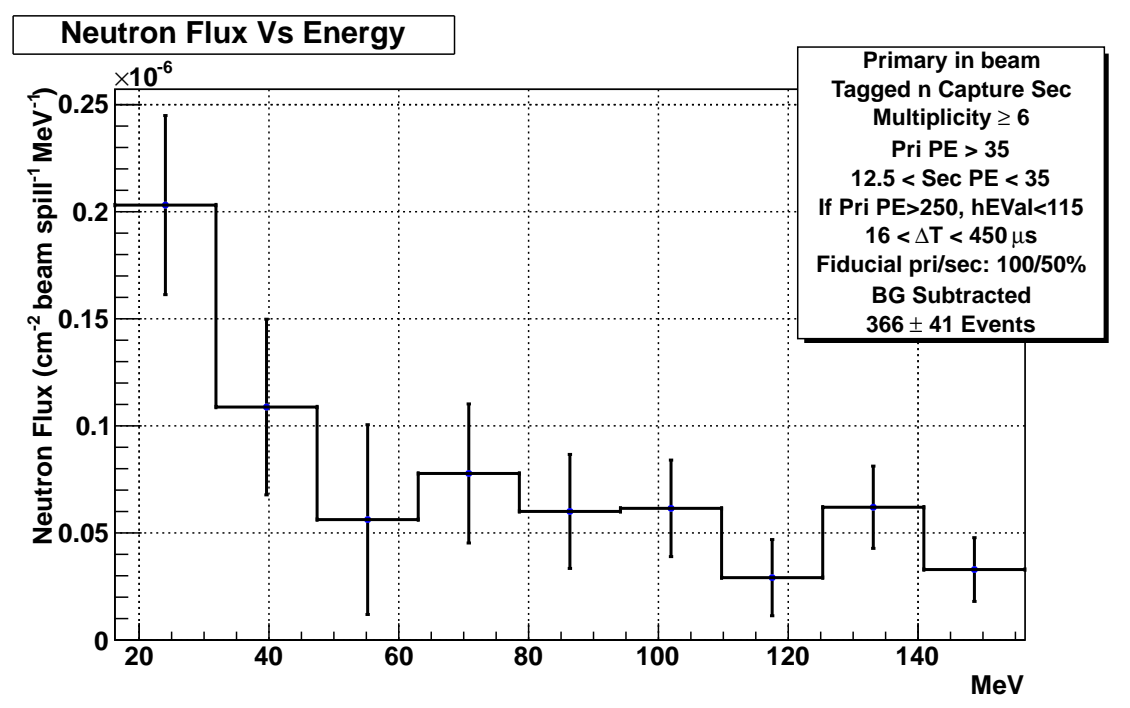

Figure 7.15: Neutron energy spectrum correlated to the NuMI beam at the MINOS hall at FNAL. The energy calibration includes the proton quenching analysis of Sec. 6.3.2, The errors are statistical and result from the background subtraction.

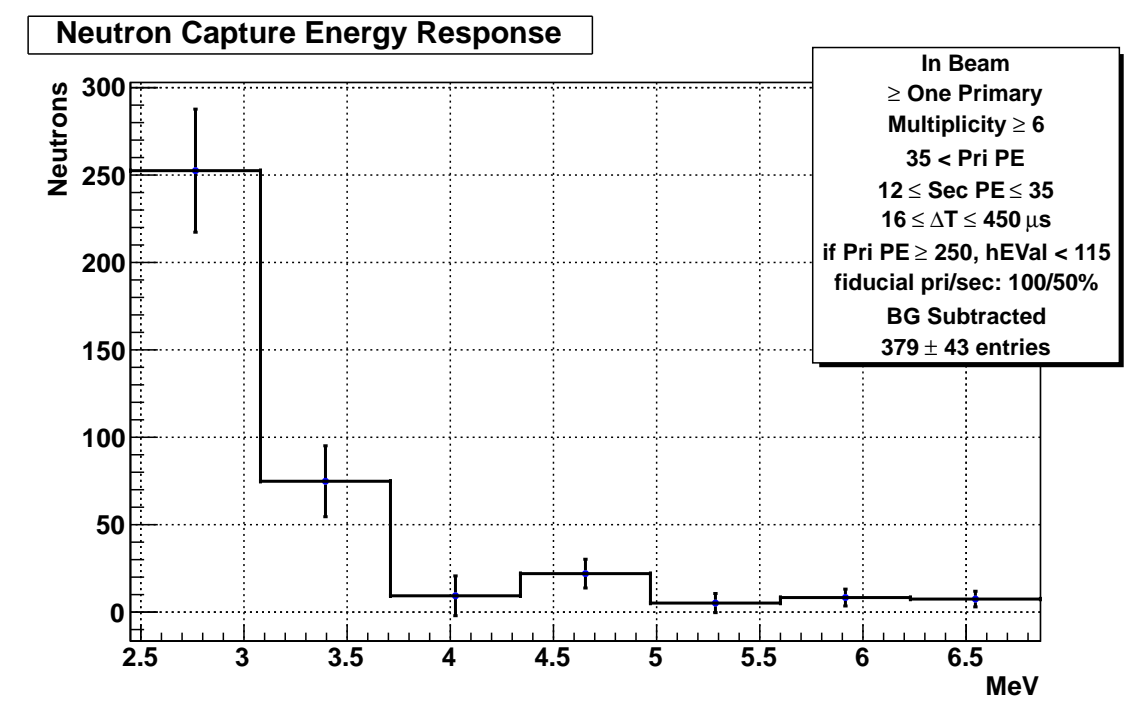

Figure 7.16: Neutron capture energy spectrum correlated to the NuMI beam at the MINOS hall at FNAL. The errors are statistical and result from the background subtraction. 
Most beta decay occurs inside the nucleus where the proton remains. The electron and neutrino are ejected from the nucleus and the electron can be detected. Double beta decay occurs when two neutrons in the same nucleus undergo this process simultaneously, releasing two electrons and two neutrinos. Neutrinoless double beta decay is a theoretical process that would occur as double beta decay except the two neutrinos would annihilate one another and only the two electrons would be ejected from the nucleus, see Fig. 7.17. Observation of this process would demonstrate violation of lepton conservation, identify neutrinos as Majorana particles, and provide a measurement of neutrino mass.

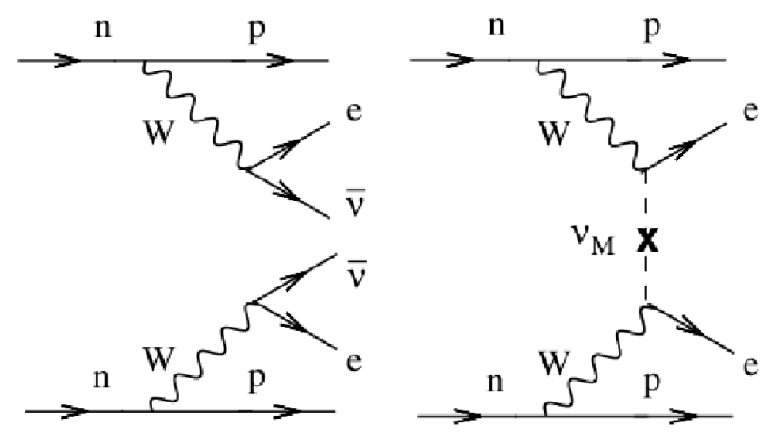

Figure 7.17: Left: double beta decay; right: neutrinoless double beta decay.

This section describes how neutrons can be a background source for the EXO neutrinoless double beta decay search and presents a rough calculation showing how our NuMI measurements could be applied to estimate their significance.

\subsubsection{Neutrons as a background for EXO}

Neutrons are a significant background to EXO because they can interact with ${ }^{136} \mathrm{Xe}$ to produce unwanted xenon isotopes ${ }^{137} \mathrm{Xe}$ and ${ }^{135} \mathrm{Xe}$, each of which can then beta decay. The production processes for these isotopes are

$$
\begin{aligned}
& { }^{136} \mathrm{Xe}+\mathrm{n} \rightarrow{ }^{135} \mathrm{Xe}+2 \mathrm{n}, \text { and } \\
& { }^{136} \mathrm{Xe}+\mathrm{n} \rightarrow{ }^{137} \mathrm{Xe}+\gamma ;
\end{aligned}
$$


and the process of beta decay is shown in Eqn. 7.9. Xenon-135 has a beta decay half life of 9.1 hours, releasing an electron of maximum energy $1.115 \mathrm{MeV}$, which is below the $2.458 \mathrm{MeV}$ neutrinoless double beta decay region of interest. Xenon-137, however, has a beta decay half life of 3.8 minutes and releases an electron of maximum energy $4.177 \mathrm{MeV}$. Xenon-137 beta decays are thus capable of mimicking the neutrinoless double beta decay signal in EXO. Figure 7.18 shows the expected contributions of all background sources at energies near the EXO region of interest with the exception of ${ }^{137} \mathrm{Xe}$, which is present over the entire energy scale at an unknown level. The ${ }^{137} \mathrm{Xe}$ background is insignificant for EXO at present, but may become more relevant for future EXO analyses and detectors.

\subsubsection{Implications of our measurements}

We would like to use our measurements at NuMI to help characterize the background fast neutron flux at WIPP, and therefore the background ${ }^{137}$ Xe contamination in EXO. Rigorous analysis of the implications of the SciBath NuMI measurements for the WIPP neutron flux are currently in progress. This section describes only a rough calculation of how our measurements imply an adjustment of the Mei and Hime [14] predicted neutron flux at WIPP. This calculation contains substantial errors and should be thought of as an exercise in how our results can be applied instead of an accurate prediction. The largest associated errors are the relatively large error on our neutron flux measurement and the unknown error incurred by extrapolating the Mei and Hime results to the NuMI depth.

Mei and Hime used a FLUKA simulation to predict muon and neutron fluxes and energy spectra at underground sites [14]. If there was a systematic error in the way the simulation handled neutron production in the rock, it would likely result in a neutron/muon flux ratio in disagreement with reality. Equation 4 in the Mei and Hime paper [14] gives the muon intensity as a function of depth, and in the case of NuMI at $0.265 \mathrm{~km}$.w.e. suggests a muon flux of $0.30 \mathrm{~m}^{-2} \mathrm{~s}^{-1}$. Similarly, Sec. 7.5 describes how Mei and Hime suggest a $1.3 \times 10^{-3} \mathrm{~m}^{-2} \mathrm{~s}^{-1}$ neutron flux. Dividing these gives the suggested neutron/muon flux ratio 

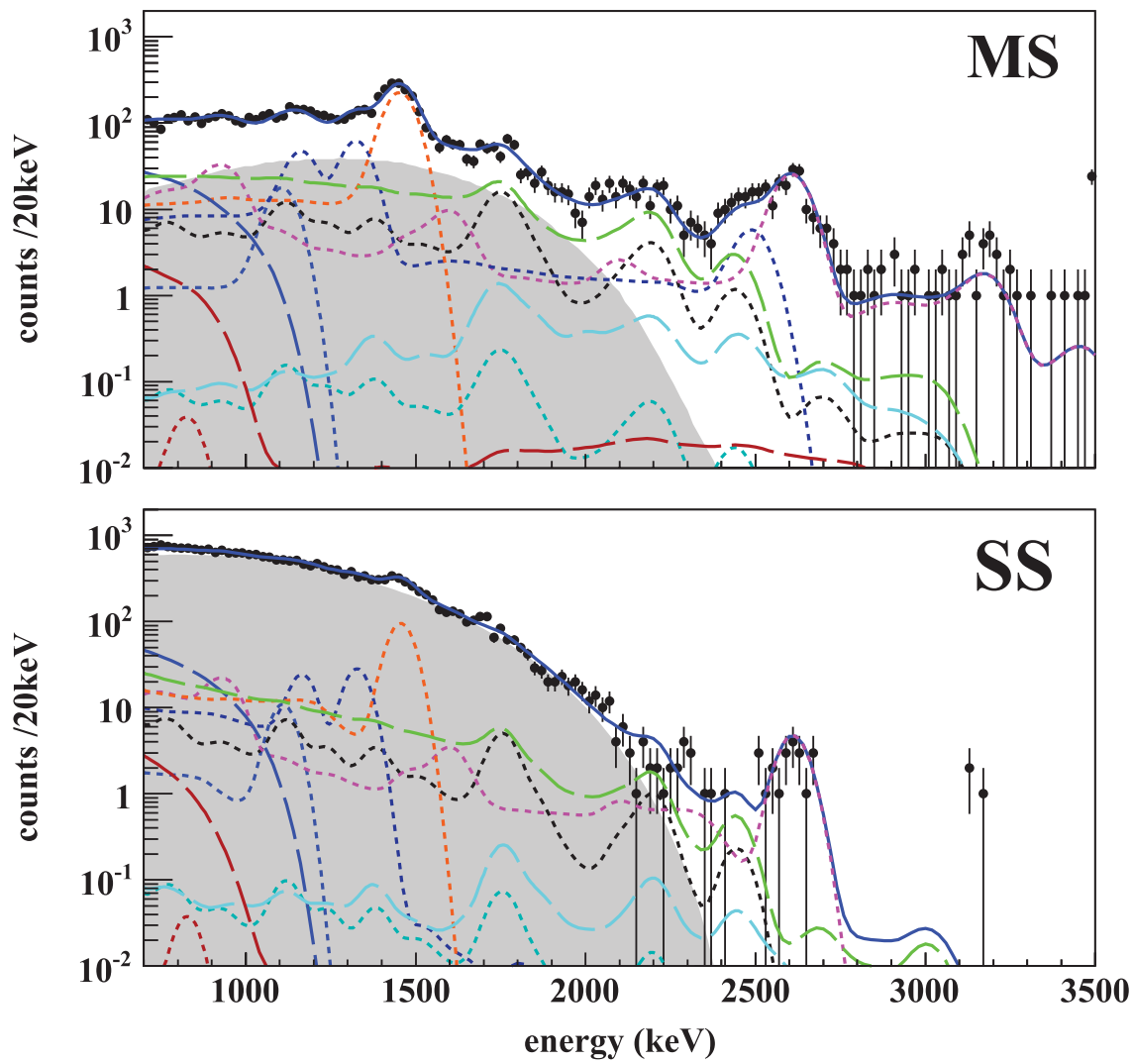

Figure 7.18: Multi-site (MS, top) and single-site (SS, bottom) energy spectra. The best-fit line (solid blue) is shown. The background components are $2 \nu \beta \beta$ (grey region), ${ }^{40} \mathrm{~K}$ (dotted orange), ${ }^{60} \mathrm{Co}$ (dotted dark blue), ${ }^{222} \mathrm{Rn}$ in the cryostat-lead air-gap (long-dashed green), ${ }^{238} \mathrm{U}$ in the TPC vessel (dotted black), ${ }^{232} \mathrm{Th}$ in the TPC vessel (dotted magenta), ${ }^{214} \mathrm{Bi}$ on the cathode (long-dashed cyan), ${ }^{222} \mathrm{Rn}$ outside of the field cage (dotted dark cyan), ${ }^{222} \mathrm{Rn}$ in active xenon (long-dashed brown), ${ }^{135} \mathrm{Xe}$ (long-dashed blue) and ${ }^{54} \mathrm{Mn}$ (dotted brown). The ${ }^{137} \mathrm{Xe}$ background component is not shown, but includes the entire energy range. The $0 \nu \beta \beta$ region of interest is at $2.458 \mathrm{MeV}$. Figure from the EXO Collaboration [15]. 
of $4.4 \times 10^{-3}$ neutrons per muon. Our measured neutron/muon flux ratio was described in Sec. 7.7 as $1.9 \times 10^{-2}$ neutrons per muon. By comparison, our measurements suggest an increase in the Mei and Hime simulated neutron/muon flux ratio by a factor of 4.3.

If we trust the Mei and Hime simulation of the muon flux at WIPP, which is validated by a measurement, then this implies the same increase in the predicted neutron flux at WIPP. The Mei and Hime result for the neutron flux at WIPP is $7.51 \times 10^{-5} \mathrm{~m}^{-2} \mathrm{~s}^{-1}$ for neutrons greater than $10 \mathrm{MeV}$, as can be read from Table $\mathrm{V}$ in [14]. Our measurements thus predict the WIPP neutron flux to be $3.2 \times 10^{-4} \mathrm{~m}^{-2} \mathrm{~s}^{-1}$ for neutrons greater than $10 \mathrm{MeV}$.

One complication that will affect the validity of this scaling argument is the difference between the muon energy at NuMI compared to WIPP. Figure 7.19 plots the energy distributions at NuMI and WIPP as predicted by Mei and Hime [14]. The correction to the neutron:muon ratio suggested by our measurements is specific to the NuMI muon energy distribution and may not directly apply to the higher energy muons at WIPP. A more rigorous investigation of the implications of our measurements on the WIPP neutron flux that takes into account the different muon energy distributions is underway.

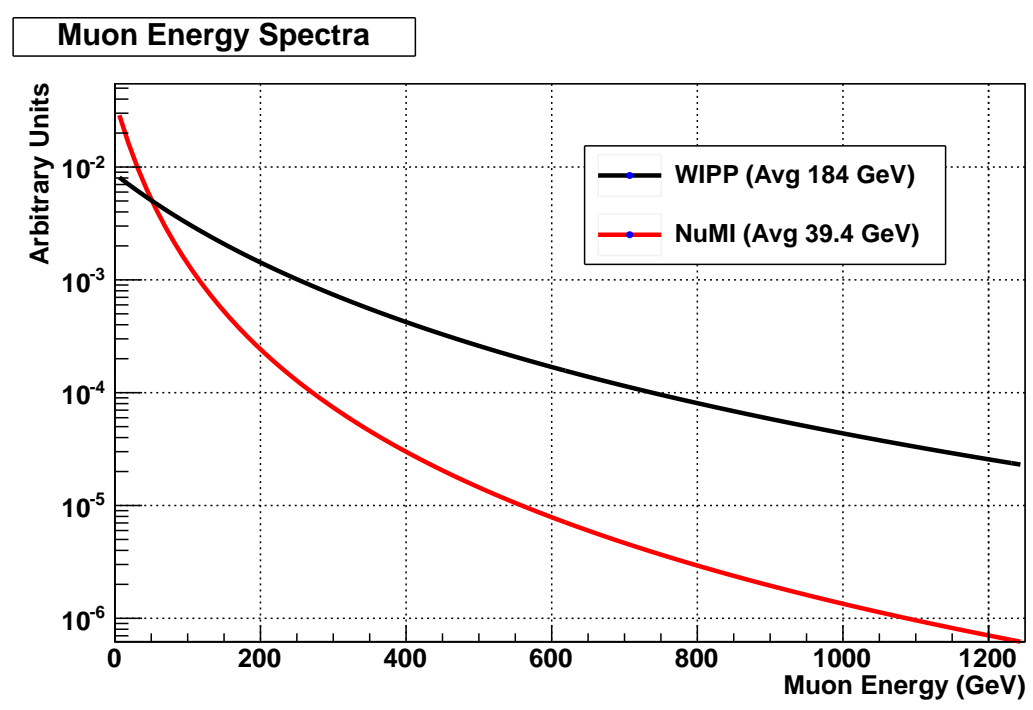

Figure 7.19: Muon energy distributions at NuMI and WIPP. Curves from Eqn. 8 of Ref. [14] with the parameters suggested by Groom et al. [1] and Battistoni et al. [16] 


\section{Chapter 8}

\section{Conclusion and Outlook}

This thesis has described the SciBath detector and its measurements of muon and neutron characteristics at $100 \mathrm{~m}$ underground from the NuMI run. The muon flux result was $0.80 \pm 0.04 \mathrm{~m}^{-2} \mathrm{~s}^{-1}$ and was in agreement with the relatively wide range of flux predictions. The muon angular distribution was presented in Fig. 7.5 and was in agreement with the Miyake prediction [13]. The neutron yield from muon-induced spallation in scintillator was $(3.8 \pm 3.2) \times 10^{-4} \mathrm{n} / \mu\left(\mathrm{g} / \mathrm{cm}^{2}\right)^{-1}$ and was high compared to similar measurements, though within error. The neutron flux at the NuMI site was $(1.5 \pm 1.4) \times 10^{-2} \mathrm{~m}^{-2} \mathrm{~s}^{-1}$ and was high compared to the Mei and Hime prediction [14, but again within error. The corresponding neutron energy distribution was presented in Fig. 7.8 and its shape is in general agreement with the Mei and Hime prediction.

Many improvements to SciBath and its analysis methodology are in progress. The Geant4 Monte Carlo simulation is being expanded to include more detailed optics, fiber specific light collection efficiencies, and modeling of the readout electronics. The largest uncertainty in our neutron measurements resulted from the efficiency of our energy cuts to identify $2.2 \mathrm{MeV}$ photons from neutron capture on hydrogen. This was largely a result of the scintillator light output being lower than expected, and studies are currently underway to understand the cause and improve the light output for future measurements. Also contributing to 
the large error, the neutron measurements of this thesis suffered from a large background subtraction for neutron capture candidates. The possibility of doping our liquid scintillator with $\mathrm{Gd},{ }^{6} \mathrm{Li}$, or ${ }^{10} \mathrm{~B}$ is under investigation. These dopants could increase the neutron capture efficiency, increase the light output by a neutron capture event, and shorten the capture time which would lower the neutron capture background. Additionally, studies of solid scintillator options are underway. Solid scintillator could simplify the detector design, make SciBath easier to operate, increase durability, reduce the possibility of oxygen poisoning, and allow a higher light yield.

Finally, improved topological analysis tools are being developed. As a fine-grained detector, the SciBath topology is perhaps its greatest strength. One idea is to look for neutron double-scattering events such as the candidate in Fig. 8.1. Identification of these events could provide a neutron angular distribution measurement. Parameters for the characterization of events as track-like or point-like based on an analytical calculation of fiber response have also been developed. These could provide an improved muon and neutron separation criteria, as shown in Fig. 8.2, and thus improve our event identification.

The SciBath detector has proven to be an effective neutral particle detector and has a lot of room for continued improvement. This first round of measurements form the detector both contributes to the understanding of backgrounds to underground experiments and provides a framework for future measurements with the SciBath detector. 

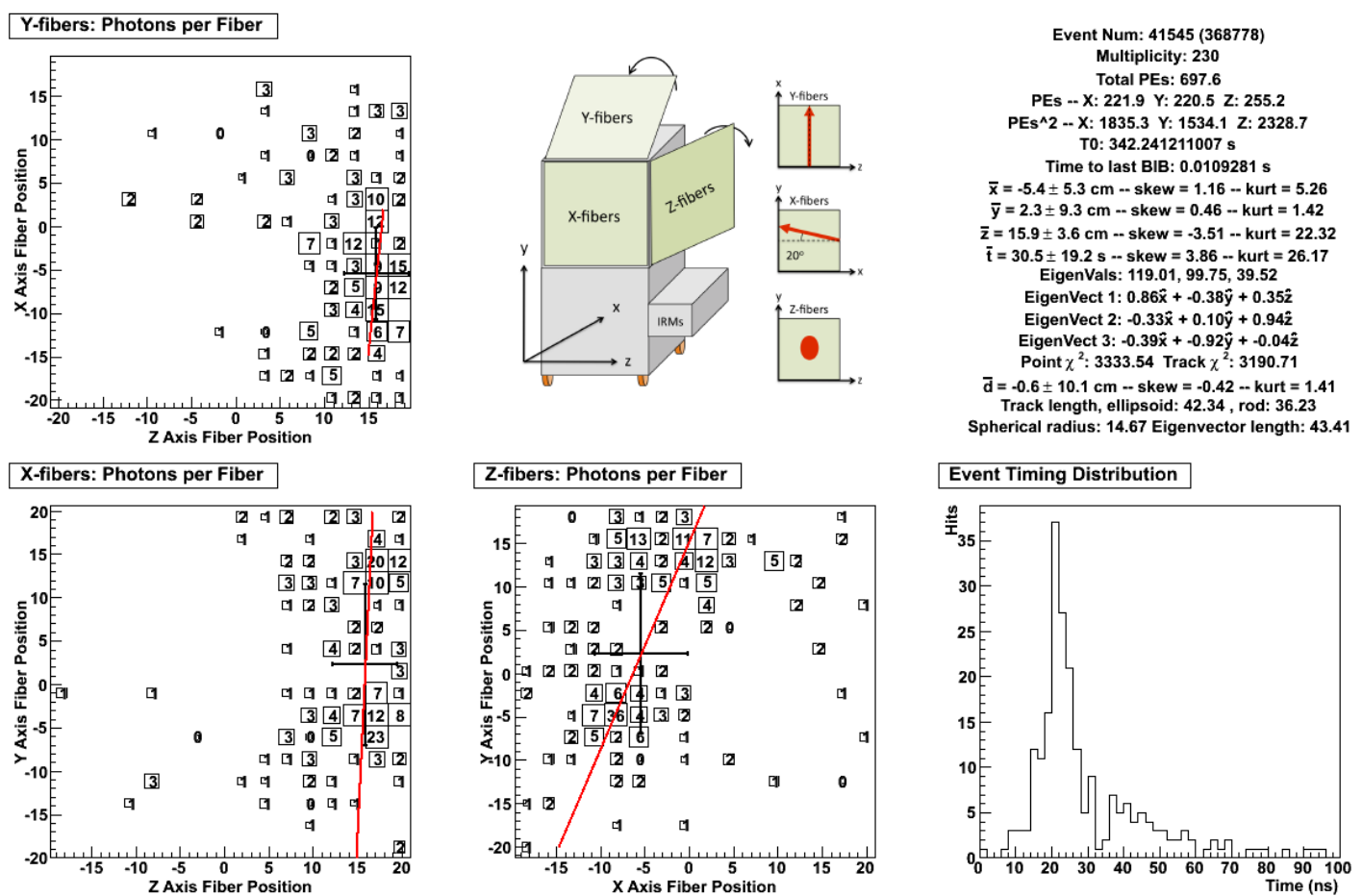

Figure 8.1: Event display for a neutron double-scattering candidate event.

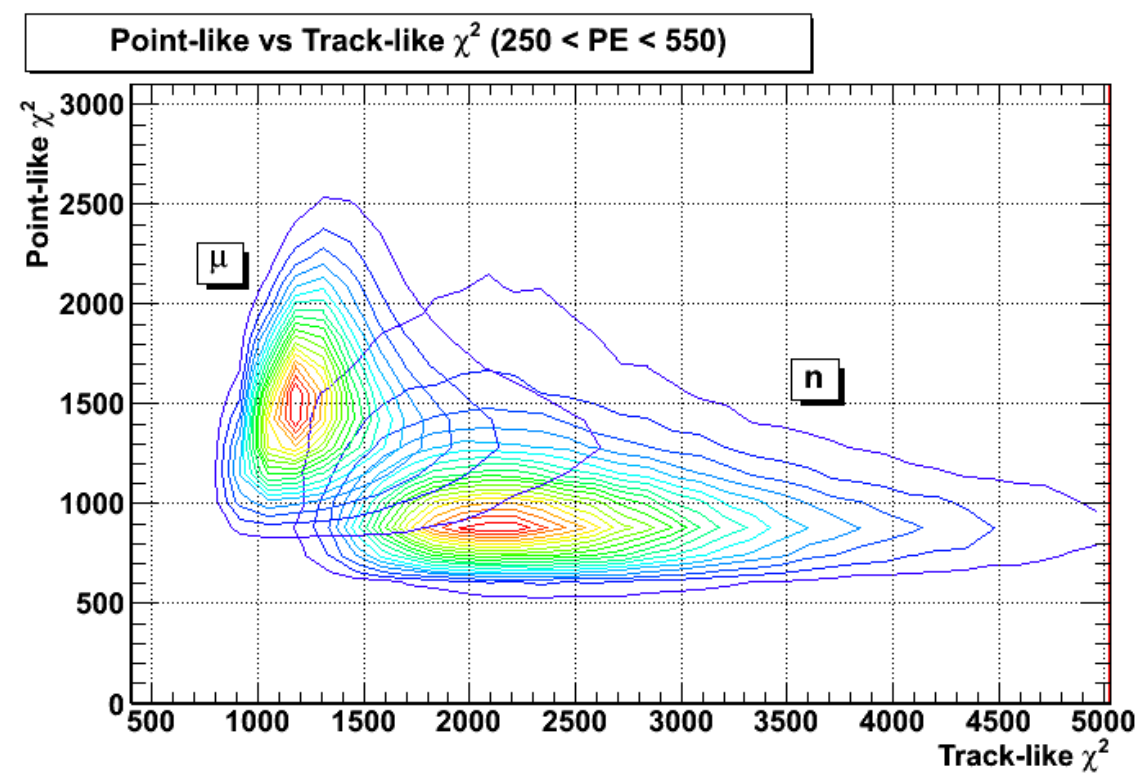

Figure 8.2: Point-like versus track-like characterization parameters for a primarily muon data set (upper left) and a primarily neutron data set (lower right), both from the MI-12 run. 


\section{Appendix A}

\section{Geant4 Physics Summary}

Version Geant4.9.4.p02

\section{Geometry}

- G4Box for the detector cube

- G4Tubs for the WLS fibers

- Environment composition is air

\section{Physics Lists}

- G4Electron

- G4Positron

- G4NeutrinoE

- G4AntiNeutrinoE

- G4PhotoElectricEffect

- G4ComptonScattering

- G4GammaConversion

- G4RayleighScattering

- G4LivermorePhotoElectricModel

- G4LivermoreComptonModel

- G4LivermoreGammaConversionModel 
- G4LivermoreRayleighModel

- G4eIonisation

- G4LivermoreIonisationModel

- G4UniversalFluctuation

- G4eBremsstrahlung

- G4LivermoreBremsstrahlungModel

- G4eMultipleScattering

- G4eplusAnnihilation

- G4MuIonisation

- G4MuBremsstrahlung

- G4MuPairProduction

- G4Decay

- HadronPhysicsQGSP_BERT_HP

- G4Scintillation

- G4OpAbsorption

- G4OpRayleigh

- G4OpBoundaryPhysics

- G4HadronElasticProcess

- G4Neutron

- G4NeutronHPorLElasticModel

- G4NeutronInelasticProcess

- G4NeutronHPorLEInelasticModel

- G4HadronCaptureProcess

- G4NeutronHPorLCapture 


\section{Appendix B}

\section{MCNP Template}

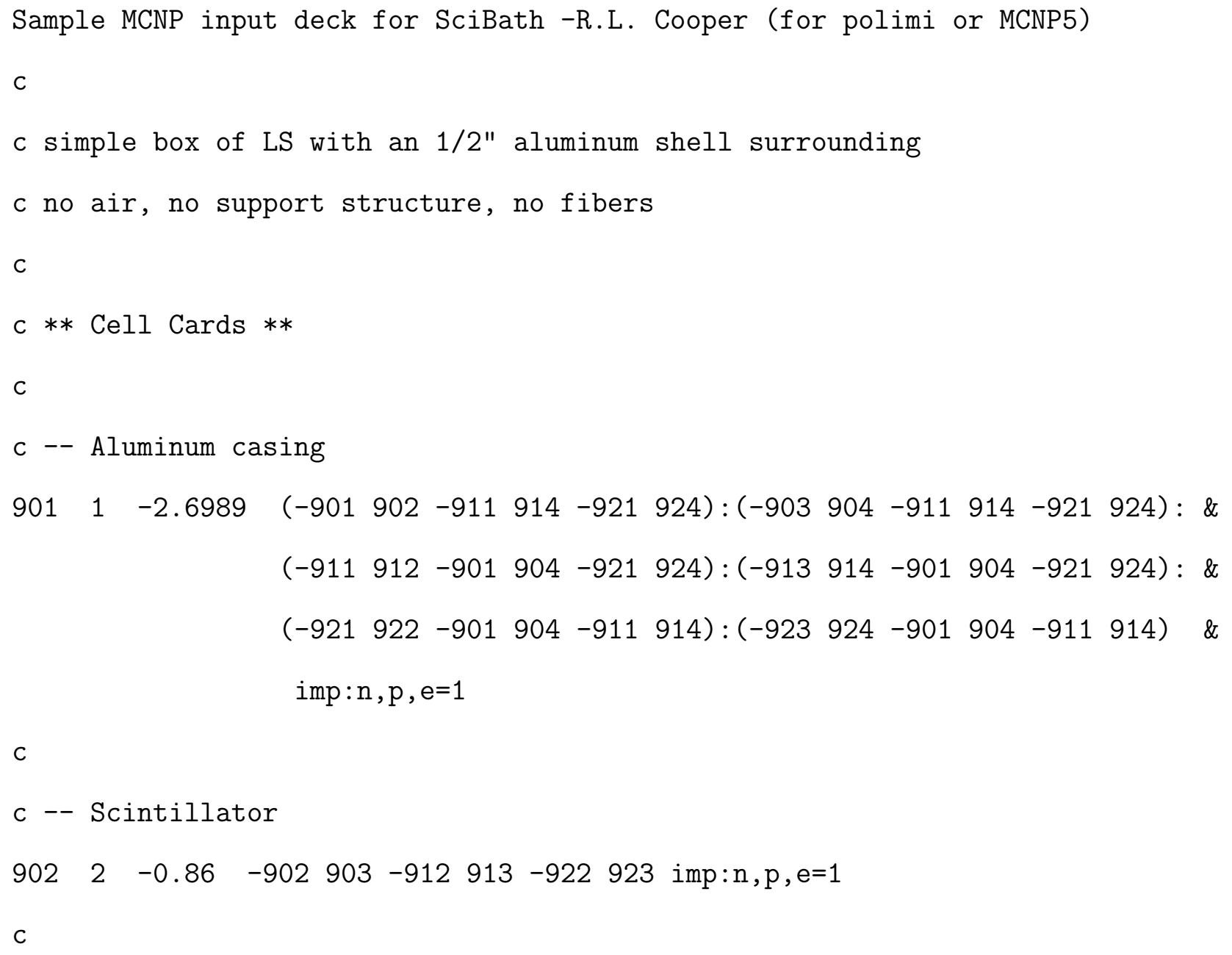




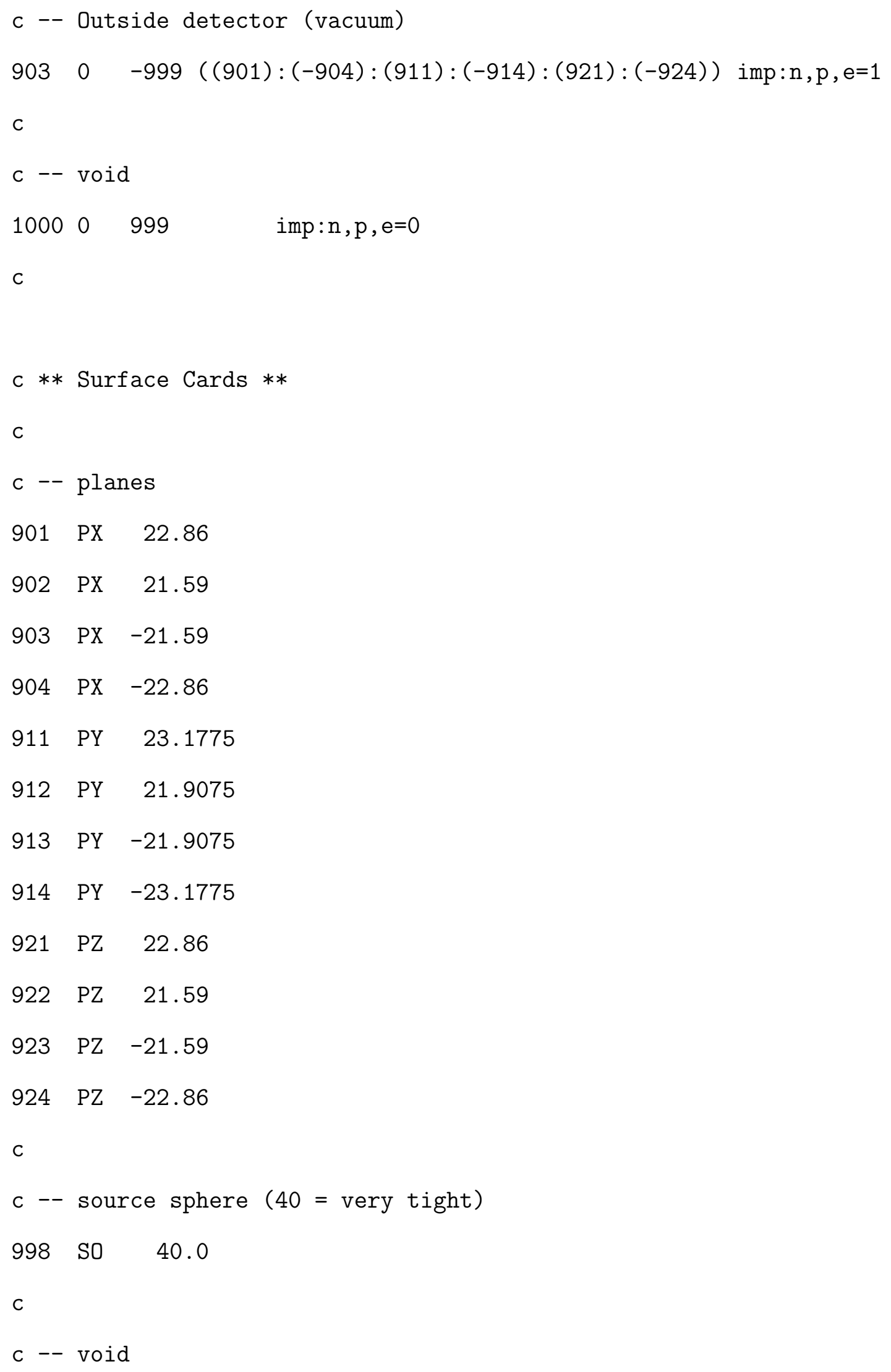




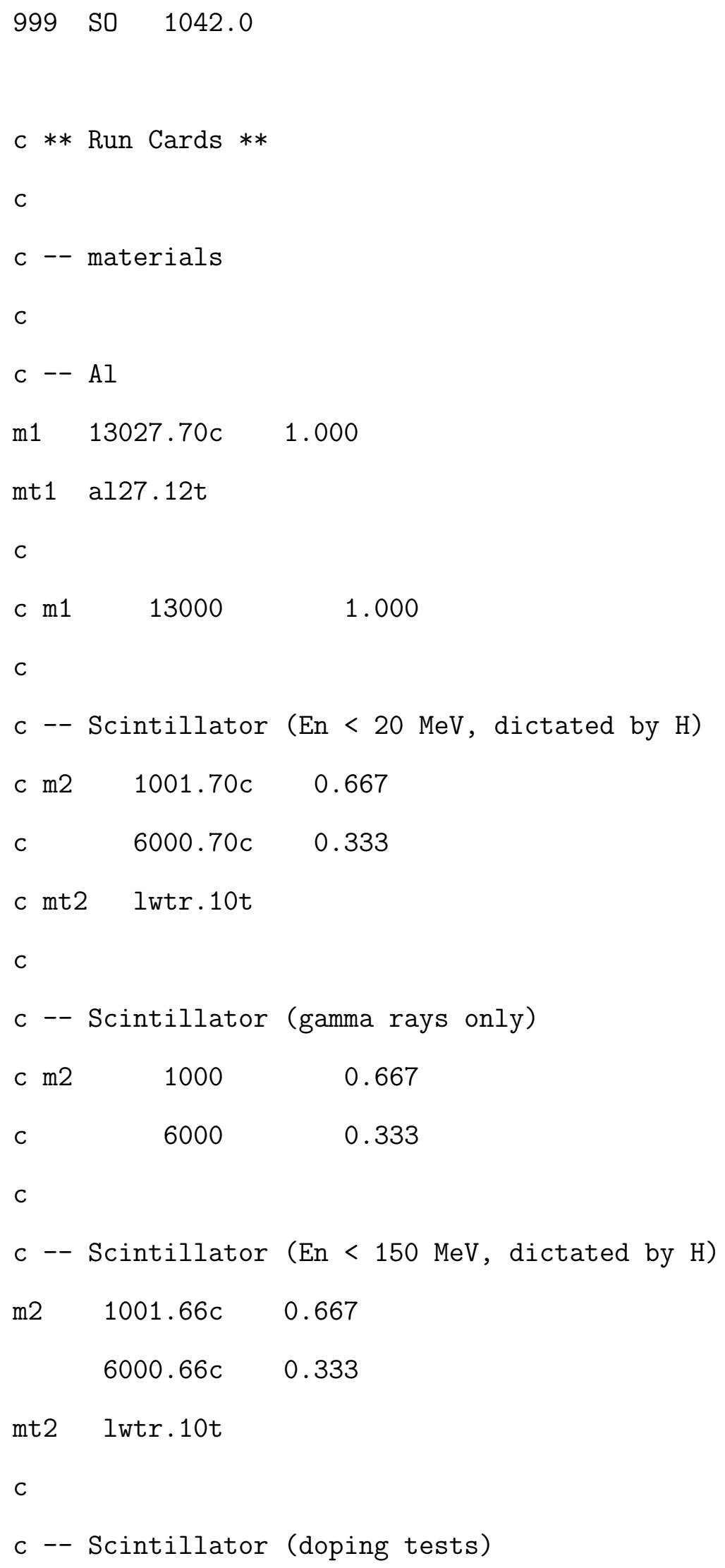




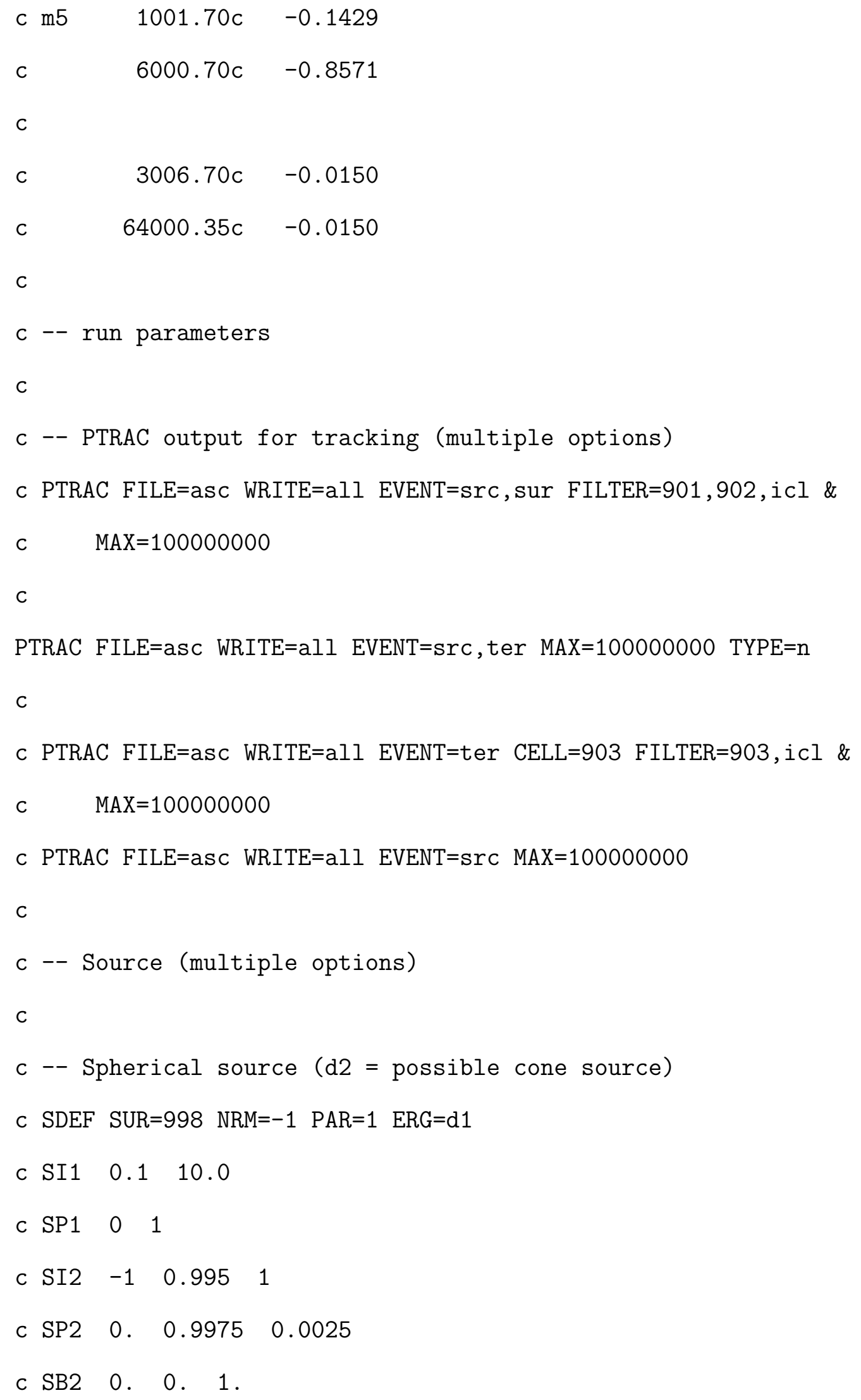




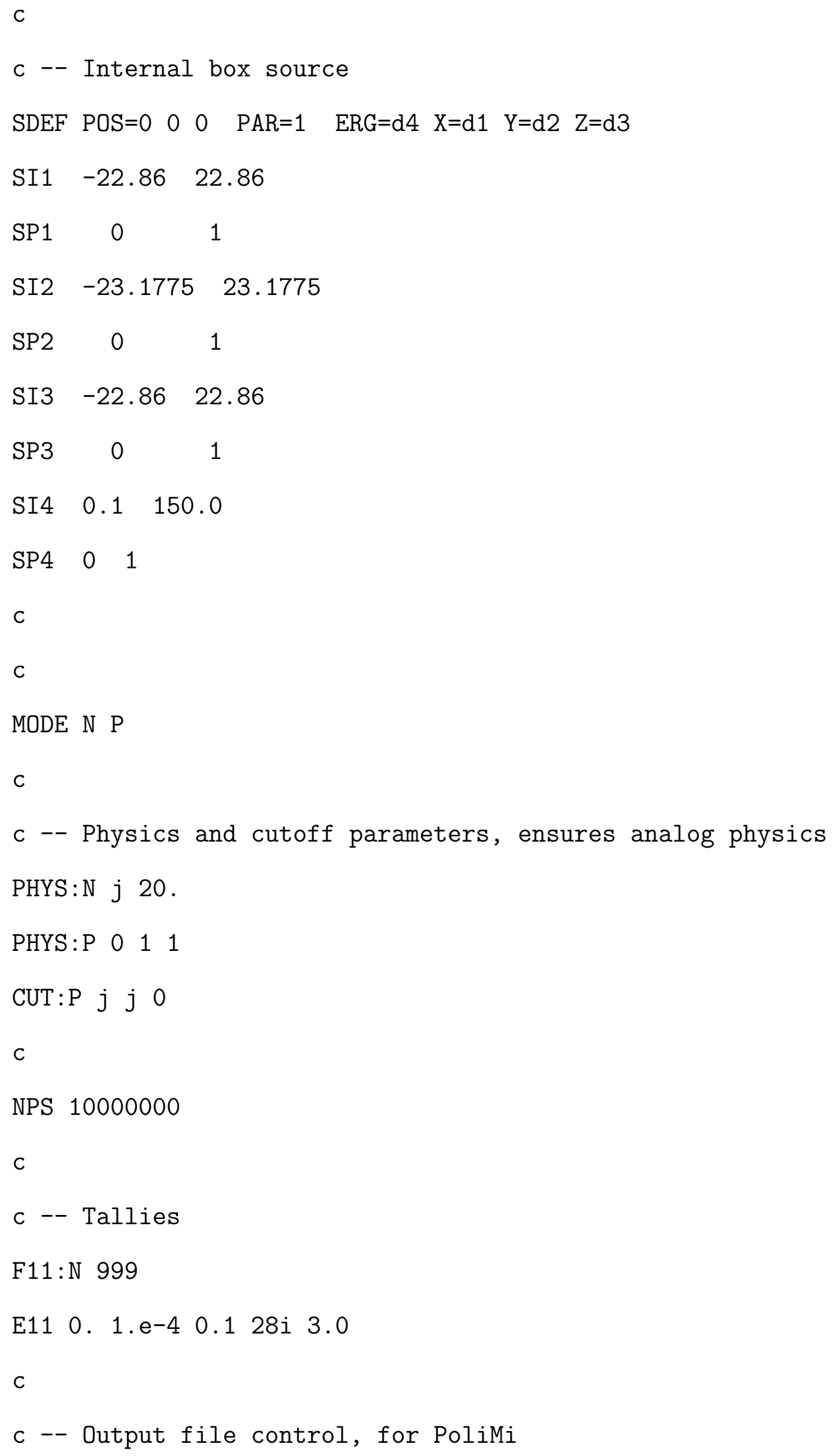




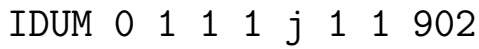

RDUM $0.01 \quad 10.05$

FILES 21 dumn 1

C

c RAND SEED $=884793$ 


\section{Appendix C}

\section{Waveform Fitting with the Least Squares Parabola Method}

IRM SciBath's data acquisition electronic boards Photomultiplier tube Analog to digital converter Least squares parabola fit method Number of samples in waveform

$T_{c}$ . Period of the electronics clock

$t_{0}$ . Start time of the hit $P E$ Charge for the waveform $A D C_{i}$ . The ADC value for the ith sample $A D C_{\text {min }}$ . The minimal ADC value $\mathcal{B}$ Baseline for the waveform $\mathcal{A}$ Fitted amplitude for the waveform $t$ Time $\tau$ Exponential decay parameter $\omega$ Angular frequency parameter 


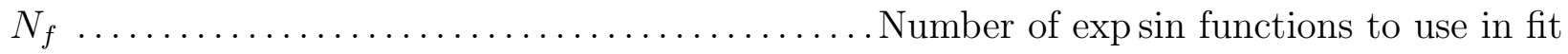
$t_{0_{i}} \ldots \ldots \ldots \ldots \ldots \ldots \ldots \ldots \ldots \ldots$ Start time of the ith function for a given waveform $\mathcal{A}_{i}$ Fitted amplitude of the ith function for a given waveform

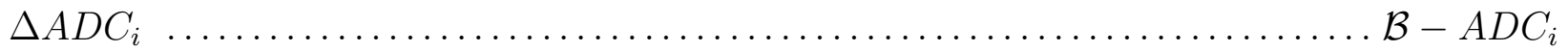

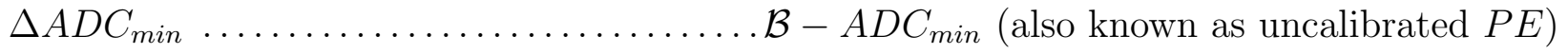

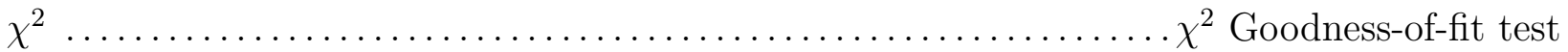
$N_{L S} \ldots \ldots \ldots \ldots \ldots \ldots \ldots \ldots \ldots \ldots \ldots$ Number of points used in the least squares fit $\underline{\theta} \ldots . .$. Column matrix that contains the resulting parameters used in a least squares fit $V(\underline{\theta}) \ldots \ldots \ldots \ldots \ldots \ldots \ldots \ldots \ldots \ldots \ldots \ldots$ Covariance matrix for parameters in $\underline{\theta}$ $\underline{A D C}$ Column matrix that contains the ADC values for the points used the least squares fit

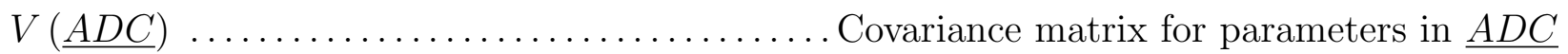
$\underline{C} \ldots \ldots \ldots \ldots \ldots \ldots \ldots \ldots \ldots \ldots \ldots$ Coefficient matrix for the equation being used $t_{i} \ldots \ldots \ldots \ldots \ldots \ldots \ldots \ldots \ldots \ldots$ The time of the ith sample in the waveform

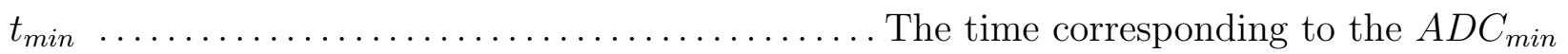

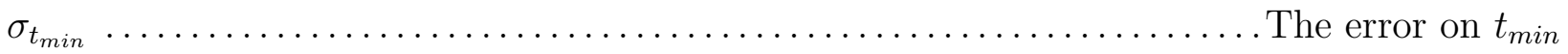

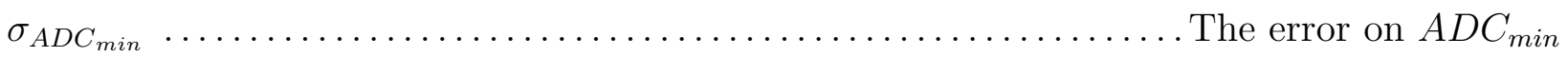

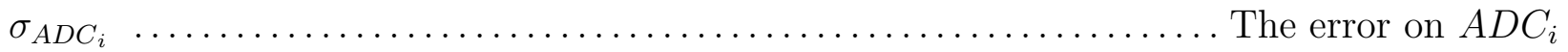


This appendix is an excerpt form an internal SciBath technote by R. T. Thornton [73].

\section{C.1 Background}

Many methods of waveform fitted were tested, but for this analysis we used the method of least squares to fit a parabola to each waveform. This method was suggested because the waveform around $A D C_{\min }$ acts like a parabola. The least squares fit must satisfy the following equation

$$
X^{2}=\sum_{i=1}^{N_{L S}} \sum_{j=1}^{N_{L S}}\left(A D C_{i}-f_{i}\right) V(\underline{A D C})_{i j}^{-1}\left(A D C_{j}-f_{j}\right)=\text { minimum }
$$

where $f_{i}$ is the fitted value at the ith point, $N_{L S}$ is the number of samples used in the fit, $V(\underline{A D C})$ is the covariance matrix for the samples used in the fit and $A D C_{i}$ is the ith samples ADC value [74]. An analytical solution to equation C.1] is only valid if $f$ is linear in parameters, which is the case for a parabola defined by the following equation:

$$
A D C=a+b t+c t^{2}=f
$$

Computationally speaking it would be easier if equation C.1 was written in matrix form, therefore, we derived the following expression[74]:

$$
\begin{aligned}
\underline{\theta} & =V(\underline{\theta}) \underline{C}^{T} V(\underline{A D C}) \underline{A D C} \\
V(\underline{\theta}) & =\left(\underline{C}^{T} V(\underline{A D C})^{-1} \underline{C}\right)^{-1}
\end{aligned}
$$


The matrix parameters in equation C.3 have the following definitions

$\underline{A D C}=\left(\begin{array}{c}A D C_{1} \\ A D C_{2} \\ \vdots \\ A D C_{N_{L S}}\end{array}\right), \underline{\theta}=\left(\begin{array}{c}\theta_{1} \\ \theta_{2} \\ \vdots \\ \theta_{L}\end{array}\right), V(\underline{A D C})=\left\{\begin{array}{ccc}\sigma_{A D C_{i}}^{2} & j=i \\ 0 & j \neq i\end{array}, \underline{C}=\left(\begin{array}{cccc}c_{11} & c_{12} & \cdots & c_{1 L} \\ c_{21} & c_{22} & \cdots & c_{2 L} \\ \vdots & \vdots & \ddots & \vdots \\ c_{N 1} & c_{N 2} & \cdots & c_{N L}\end{array}\right)\right.$

where $L$ is the number of parameters in the fit (for LSP $L=3$ ), $\underline{\theta}$ is the resulting parameter matrix, $V(\underline{\theta})$ is the covariance matrix for the resulting parameters, $\underline{A D C}$ is the matrix of the ADC values for the samples used in the fit, and $\underline{C}$ is the coefficient matrix for the fitted function [74].

\section{C.2 Implementation}

To implement this method, we choose to use ROOT's TMatrix class (GNU also has a matrix class that could be used, if independence from ROOT is needed). The method can be summarized in the following steps:

1. Calculate $\mathcal{B}$ and retrieve index of first sample above threshold, $i_{\text {start }}$

2. Loop through the samples starting at $i_{\text {start }}$ through $N_{T}-3$ finding $A D C_{m i n}$, and its corresponding index, $i_{\text {min }}$. The minus 3 is to use the same method as Reader.

3. The user should have provided $N_{L S}$ (if not the default is 4 ). Start looping through the samples at $i_{\text {min }}-2$ storing samples that satisfy $i>=i_{\text {start }}$ till the number of samples received is equal to $N_{L S}$. 
4. Calculate

$$
\underline{C}=\left(\begin{array}{ccc}
1 & t_{1} & t_{1}^{2} \\
1 & t_{2} & t_{2}^{2} \\
\vdots & \vdots & \vdots \\
1 & t_{N_{L S}} & t_{N_{L S}}^{2}
\end{array}\right)
$$

where $t_{i}=i * T_{c}$, then calculate

$$
V(\underline{A D C})= \begin{cases}\min \left(1,0.3 \sqrt{\left|\Delta A D C_{i}\right|}\right)^{2} & j=i \\ 0 & j \neq i\end{cases}
$$

where $\triangle A D C_{i}=\mathcal{B}-A D C_{i}$. Then calculate equation C.3.

5. At this point the parameters for the equation of the parabola are known as well as it covariance matrix. To be able to solve for $t_{0}$ and $\mathcal{A}$ we assume that we know what $\tau$ and $\omega$ are. By knowing these two parameters, we can use equation C.7 to solve for $t_{0}$ and then use equation $\mathrm{C} .8$ to solve for $\mathcal{A}$.

$$
\begin{aligned}
& t_{0}=t_{\text {min }}-t_{\text {shift }} \\
& \mathcal{A}=\frac{\Delta A D C_{\text {min }}}{\exp \left(\frac{t_{\text {shift }}}{\tau}\right) \sin \left(\omega t_{\text {shift }}\right)}
\end{aligned}
$$

where

$$
t_{\text {shift }}=\frac{\arctan (\tau \omega)}{\omega} \Delta A D C_{\text {min }}=\mathcal{B}-A D C_{\text {min }}
$$

where $t_{\min }$ is the time of the minimal value given by the parabola fit with $A D C_{\text {min }}$ as its ADC value, and they are calculated by using the following functions

$$
\begin{aligned}
t_{\text {min }} & =-\frac{\theta_{2}}{2 \theta_{3}} \\
A D C_{\text {min }} & =\theta_{1}+\theta_{2} t_{\text {min }}+\theta_{3} t_{\text {min }}^{2}
\end{aligned}
$$


and the errors on $t_{\min }$ and $A D C_{\min }$ were calculated with the following functions

$$
\begin{gathered}
\sigma_{t_{\text {min }}}=t_{\min } \sqrt{\frac{V(\underline{\theta})_{22}}{\theta_{2}^{2}}+\frac{V(\underline{\theta})_{33}}{\theta_{3}^{2}}-\frac{2 V(\underline{\theta})_{23} \theta_{3}}{\theta_{2}}} \\
\sigma_{A D C_{\text {min }}}=\sqrt{V(\underline{\theta})_{11}-\frac{V(\underline{\theta})_{12} \theta_{2}}{\theta_{3}}+\frac{V(\underline{\theta})_{13} \theta_{2}^{2}}{2 \theta_{3}^{2}}+\frac{V(\underline{\theta})_{22} \theta_{2}^{2}}{4 \theta_{3}^{2}}-\frac{V(\underline{\theta})_{23} \theta_{2}^{3}}{4 \theta_{3}^{3}}+\frac{V(\underline{\theta})_{33} \theta_{2}^{4}}{16 \theta_{3}^{4}}}
\end{gathered}
$$

6. The last step that is needed is to calculate a goodness-of-fit value. We chose to keep with the $\chi^{2}$ test, which is defined with respect to the exp sin function.

\section{C.3 Results}

As mentioned above in order for this method to work $\tau$ and $\omega$ already need to be known. We assumed that all fibers have the same values for both parameters and the values are $\tau=242.0575 \mathrm{~ns}$ and $\omega=0.007851405 \frac{1}{\mathrm{~ns}}$, which were calculated by taking an average of the average channel values. Figure 6.1 shows the visual results of the LSP method. 


\section{Bibliography}

[1] D. E. Groom, N. V. Mokhov, and S. I. Striganov, Atomic Data and Nuclear Data Tables 78, 183 (2001).

[2] N. Y. Agafonova and A. S. Malgin, Physical Review D: Particles, Fields, Gravitation \& Cosmology 87, 1 (2013).

[3] ICRU Report No. 49, (1993), [Tables and graphs of these data are available at http:// physics.nist.gov/PhysRefData/].

[4] W. H. Barkas, W. Birnbaum, and F. M. Smith, Phys. Rev. 101, 778 (1956).

[5] H. Bichsel, Phys. Rev. A 41, 3642 (1990).

[6] E.-I. Esch, Detector Development for Dark Matter Research, Ph.D. thesis, Justus-LiebigUniversität Giessen (2001).

[7] A. Malgin and O. Ryazhskaya, Phys. Atom. Nucl. 71, 1769 (2008).

[8] M. Chadwick, M. Herman, P. Obloinsk, et al., Nuclear Data Sheets 112, 2887 (2011).

[9] A. Del Guerra, Nucl. Instrum. Meth. 135, 337 (1976).

[10] M. Anghinolfi, G. Ricco, P. Corvisiero, and F. Masulli, Nucl. Instrum. Meth. 165, 217 (1979).

[11] M. Berger, J. Hubbell, S. Seltzer, et al., http://www.nist.gov/pml/data/xcom/ index.cfm. 
[12] A. Dragić, D. Jokovi, R. Banjanac, et al., Nucl. Instrum. Meth. A 591, 470 (2008).

[13] S. Miyake, International Cosmic Ray Conference 5, 3638 (1973), Denver 1973.

[14] D. Mei and A. Hime, Phys. Rev. D 73, 053004 (2006), arXiv:astro-ph/0512125 [astro-ph]

[15] M. Auger, D. J. Auty, P. S. Barbeau, et al. (EXO Collaboration), Phys. Rev. Lett. 109, 032505 (2012).

[16] G. Battistoni et al. (MACRO Collaboration), To be published in the proceedings of Conference: C98-05-25.2 , 419 (1998), arXiv:hep-ex/9809006 [hep-ex] .

[17] F. Boehm, J. Busenitz, B. Cook, et al., Phys. Rev. Lett. 84, 3764 (2000), arXiv:hepex/9912050 [hep-ex] .

[18] M. Apollonio, A. Baldini, C. Bemporad, et al., Physics Letters B 466, 415 (1999).

[19] R. Alivisatos et al. (KamLAND Collaboration), Report No. Stanford-HEP-98-03, RCNS-98-15 (1999).

[20] S. Golwala, R. Abusaidi, D. Akerib, et al., Nucl. Instrum. Meth. A 444, 345 (2000),

[21] W. Leo, Techniques for Nuclear and Particle Physics Experiments: A How-To Approach (Springer-Verlag, 1994).

[22] R. D. Evans, TheAtomic Nucleus (McGmw-Hill Book Co., New York, 1955).

[23] D. Madland and J. Nix, Nucl. Sci. Eng. 81:2, 213 (1982).

[24] J. Terrell, Phys. Rev. 127, 880 (1962).

[25] P. Singer, Springer Tracts in Modern Physics (1974).

[26] W. U. Schröder, Ph.D. thesis, Technisch Hochschule Darmstadt (1971). 
[27] M. H. Krieger, Ph.D. thesis, Columbia University, NEVIS-172 (1969).

[28] A. I. Barbouti and B. C. Rastin, Journal of Physics G: Nuclear Physics 9, 1577 (1983).

[29] L. N. Bogdanova, M. G. Gavrilov, V. N. Kornoukhov, and A. S. Starostin, Physics of Atomic Nuclei 69, 1293 (2006).

[30] S. Kasahara, NuMI-277r, Tech. Rep. (MINOS Collaboration) [http://homepages.spa.umn.edu/ schubert/near/].

[31] J. Sullivan, Nucl. Instrum. Meth. 95, 5 (1971).

[32] O. Allkofer, G. Bella, W. Dau, et al., Nuclear Physics B 259, 1 (1985).

[33] R. Hertenberger, M. Chen, and B. Dougherty, Phys. Rev. C 52, 3449 (1995).

[34] F. Boehm, J. Busenitz, B. Cook, et al., Phys. Rev. D 62, 092005 (2000), arXiv:hepex/0006014 [hep-ex] .

[35] L. B. Bezrukov et al., Soviet Journal of Nuclear Physics 17, 51 (1973).

[36] O. G. Ryazhskaya, Diss doctor INR RAS, Moscow (1986).

[37] S. Abe et al. (KamLAND Collaboration), Phys. Rev. C 81, 025807 (2010), arXiv:0907.0066 [hep-ex] .

[38] N. Y. Agafonova et al. (LVD Collaboration), Izv. Ross. Akad. Nauk Ser. Fiz. 69, 400 (2005), [Bull. Russ. Akad. Sci. Phys. 69,. (2005)].

[39] R. Persiani, Ph.D. thesis, University of Bologna (2011).

[40] M. Aglietta, G. Badino, G. Bologna, et al., Nuovo Cim. C 12, 467 (1989).

[41] A. Lindote, H. Araujo, V. Kudryavtsev, and M. Robinson, Astropart. Phys. 31, 366 (2009), arXiv:0810.1682 [hep-ex] . 
[42] M. Aglietta et al. (LVD Collaboration), (1999), arXiv:hep-ex/9905047 [hep-ex] .

[43] V. Chazal, F. Boehm, B. Cook, et al., Nucl. Instrum. Meth. A 490, 334 (2002), arXiv:hep-ex/0102028 [hep-ex] .

[44] Y. Wang, V. Balic, G. Gratta, et al., Phys. Rev. D 64, 013012 (2001), arXiv:hepex/0101049 [hep-ex].

[45] P. Lipari and T. Stanev, Phys. Rev. D 44, 3543 (1991).

[46] L. Bugel et al. (FINeSSE Collaboration), (2004), arXiv:hep-ex/0402007 [hep-ex] .

[47] Eljen Technology, Sweetwater, TX 79556, www.eljentechnology.com/, accessed Mar. 5, 2013.

[48] Saint-Gobain Crystals, http://www.detectors.saint-gobain.com/, accessed Mar. 5, 2013.

[49] R. Tayloe, H. Meyer, D. Cox, A. Doskow, J.and Ferguson, et al., Nucl. Instrum. Meth. A 562, 198 (2006).

[50] Sigma-Aldrich, http://www.sigmaaldrich.com (2013), accessed Nov. 29, 2013.

[51] Hamamatsu Corporation, Hamamatsu City, Japan, http://www.hamamatsu.com/, accessed Mar. 5, 2013.

[52] Swagelok, http://www.swagelok.com/.

[53] Fluid-o-Tech, http://www.fluidotech.com/, accessed Dec. 2, 2013.

[54] New Pig, http://www.newpig.com/, accessed Dec 2, 2013.

[55] A. Wehmann, Concentration Levels of Inflow in NuMI Sump Water, Tech. Rep. (FNAL, 1999) nuMI-B-494.

[56] S. Brice, R. Cooper, F. DeJongh, et al., arXiv:1311.5958 (2013). 
[57] B. Braizinha, J. Esterline, H. Karwowski, and W. Tornow, Nucl. Instrum. Meth. A 623, 1046 (2010).

[58] J. Birks, The theory and practice of scintillation counting, International series of monographs on electronics and instrumentation (Macmillan, 1964).

[59] C. N. Chou, Phys. Rev. 87, 904 (1952).

[60] M. Berger, J. Coursey, M. Zucker, and J. Chang, "Estar, pstar, and astar: Computer programs for calculating stopping-power and range tables for electrons, protons, and helium ions (version 1.2.3)," [Online] Available: http://physics.nist.gov/Star [2012, August 31] (2005).

[61] S. Agostinelli, J. Allison, K. Amako, et al., Nucl. Instrum. Meth. A 506, 250 (2003).

[62] J. Allison, K. Amako, J. Apostolakis, et al., IEEE Transactions on Nuclear Science 53, $270(2006)$.

[63] MCNP, https://mcnp.lanl.gov/.

[64] F. B. Brown et al., (2003), a General Monte Carlo N-Particle Transport Code, Version 5 LA-UR-03-1987.

[65] S. A. Pozzi, E. Padovani, and M. Marseguerra, Nucl. Instrum. Meth. A 513, 550 (2003).

[66] ENDF, https://www-nds.iaea.org/exfor/endf.htm.

[67] A. Da Silva, B. Pritychenko, B. Dougherty, et al., Nucl. Instrum. Meth. A 354, 553 $(1995)$.

[68] J. Formaggio and C. Martoff, Ann. Rev. Nucl. Part. Sci. 54, 361 (2004).

[69] G. V. Gorshkov and V. A. Zyabkin, Soviet Atomic Energy 34(3), 269 (1973).

[70] K. Zbiri, Nucl. Instrum. Meth. A 615, 220 (2010). 
[71] L. A. Rebenitsch, NuMI muon rates and angular distribution at the MINOS neardetector site, Tech. Rep. (Indiana University, 2012) [Elog: Lori_Rebenitsch/150, SciBath_analysis/378].

[72] EXO Collaboration, http://www-project.slac.stanford.edu/exo/.

[73] R. T. Thornton, SciBath Waveform Fitting Documentation, Tech. Rep. (Indiana Univeristy, 2012) [Elog: Tyler_Thornton/42].

[74] A. G. Frodesen, O. Skjeggestad, and H. Tøfte, Probability and statistics in particle physics, Vol. 1 (Universitetsforlaget, 1979) 


\section{Lance Garrison}

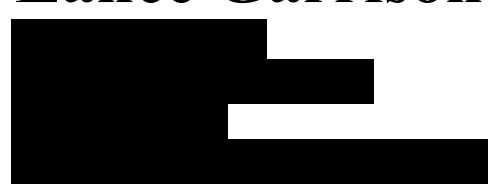

\section{CAREER OBJECTIVE}

Ensure the safe use and maintenance of nuclear materials by applying my nuclear physics education to a position at the intersection between nuclear science and policy.

\section{EDUCATION}

Ph.D., Nuclear Physics, Indiana University, Bloomington, IN, 02/30/2014

Preliminary Thesis: Measurement of cosmic neutron and muon fluxes $100 \mathrm{~m}$ underground with the SciBath detector Adviser: Dr. Rex Tayloe

B.S., Physics, University of Missouri, Columbia, MO, 2007

B.S., Mathematics, University of Missouri, Columbia, MO, 2007

\section{PHYSICS RESEARCH EXPERIENCE}

Research Assistant, Indiana University, Bloomington, Indiana, 2008-Present

- Built, commissioned, and operated SciBath-768, a neutral particle detector

- Analyzed large data sets resulting in a measurement of neutron characteristics underground

Research Assistant for College Credit, University of Missouri, Columbia, Missouri, 2006-2007

- Analyzed condensed matter physics data with statistical methods

Research Assistant, Research Experience for Undergraduates,

Northwestern University Materials Research Science and Engineering Center, Evanston, Illinois, 2005

- Developed statistical simulations of theoretical models of materials

\section{RELATED COURSEWORK AND WORKSHOPS}

Short Course on Nuclear Weapons Issues, George Washington University, Washington, DC, Nov 2-3, 2013

The American Physical Society: Forum on Physics and Society

Topics include: arms control, the nuclear-test ban treaty, nuclear proliferation, mass casualty terrorism, and ballistic missile defense

Energy Research Opportunities Workshop, Baltimore, Maryland, March 17, 2013

Topics include: the electric grid, energy storage, renewable energy science and technology, photovoltaics

Environmental Policy, Indiana University, Bloomington, Indiana, Fall 2012

Topics include: energy, air pollution, toxic wastes, federal lands, global environmental issues including climate change, congressional action, executive action, and environmental law

University Science Teaching, Indiana University, Bloomington, Indiana, Fall 2009

Topics include: differentiation, conceptual change, active learning in laboratory and lecture settings, assessment, classroom management, classroom planning, and classroom technology

\section{INTERNSHIP}

Research Intern, Arms Control Association, Washington, DC, spring 2014

Topics will include: Nuclear non-proliferation, Comprehensive Nuclear Test-Ban Treaty 


\section{TEACHING EXPERIENCE}

Assistant Instructor, Physics Department, Indiana University, Bloomington, Indiana, 2007-2010

- Taught over 400 students in 13 physics laboratory or discussion sections

- Graded student exams, quizzes, papers, and laboratory exercises

Summer Program Cofounder, Administrator, and Instructor, 2010-Present

Foundations in Science and Mathematics, Indiana University, Bloomington, Indiana

- Taught 42 local high school students over 84 hours in physics, advanced physics, and calculus

- Developed innovative inquiry-based curriculum

- Evaluated program effectiveness through testing, grading, and analysis of student performance

- Assisted in grant-writing, securing $\$ 30,000$ in grants and university funds

- Advertised the program by posting fliers, working with local high schools, maintaining a facebook.com page, and offering information sessions for parents

Mathematics Tutor, The Learning Center, University of Missouri, Columbia, Missouri, 2004-2007

- Tutored students in algebra, pre-calculus, calculus, and business calculus

- Tutored 40 students over 1000 hours in one-on-one sessions

- Lead 400 hours of group tutoring sessions open to all undergraduate mathematics students

Assistant Instructor, Physics Department, University of Missouri, Columbia, Missouri, 2007

- Taught 40 students in two physics laboratory sections

- Graded student exams and laboratory exercises

\section{SELECTED PRESENTATIONS AND PUBLICATIONS}

"A method for measuring coherent elastic neutrino-nucleus scattering at a far off-axis high-energy neutrino beam target," Physical Review D 89, 7 (2014)

"A Summer Math and Physics Program for High School Students: Student Performance and Lessons Learned in the Second Year," The Physics Teacher 51, 280 (2013)

"A Measurement of Underground Neutral Particle Fluxes With the SciBath Detector," APS April Meeting, 2012

"SciBath: A Novel Tracking Detector for Neutral Particles," APS Division of Nuclear Physics Meeting, 2010

\section{SELECTED FELLOWSHIPS AND AWARDS}

American Physical Society Travel Award, American Physical Society April Meeting, 2012

Graduate Assistance in Areas of National Need Fellowship for Science Teaching, 2007-2010

Missouri Higher Education Academic Scholarship, 2002-2007

Excellence Award Scholarship, University of Missouri, 2002-2007

Paul E. Basye Scholarship, Physics Department, University of Missouri, 2006-2007

Newell S. Gingrich Scholarship, Physics Department, University of Missouri, 2004-2005

Donald L. and Lona Lewis Packwood Endowed Undergraduate Scholarship, Physics Department, University of Missouri, 2003-2004

\section{VOLUNTEER ACTIVITIES}

Martial Arts Instruction, Ryukyu Kyusho Dojo, Bloomington, Indiana, 2009-2013

Open House Volunteer, Department of Physics, Indiana University, Bloomington, Indiana, 2007-2012

Painter, Earning endowment for Melvin Y. Mora Scholarship Fund, Department of Physics, University of Missouri, Columbia, Missouri, 2004-2006

Open House Volunteer, Department of Physics, University of Missouri, Columbia, Missouri, 2004-2006 


\section{ORGANIZATIONS}

American Physical Society

American Geophysical Union

Greenpeace USA

Society of Physics Students

University of Missouri chapter offices: President 2005-2007, Treasurer 2004-2005 